\title{
Another look at human granulocyte kinetics
}


Another look at human granulocyte kinetics

Marwan Hassani 
Another look at human granulocyte kinetics

PhD dissertation, Utrecht University

(C) 2020 Marwan Hassani

ISBN: 978-94-6332-649-9

Cover artwork: Mark Koppenberg

Lay-out: Ineke Jansen, GVO drukkers \& vormgevers B.V

Printed by: www.proefschriften.nl

Part of the research in this thesis (The Footstep study) was supported by an ISS grant from GlaxoSmithKline (GSK).

Printing of this thesis was kindly supported by Infection \& Immunity Utrecht.

All rights reserved. No part of this thesis may be reproduced, stored in a retrieval system, or transmitted, in any form or by any means, electronically, mechanically, by photo-copying, recording or otherwise, without the prior written permission of the author. 


\title{
Another look at human granulocyte kinetics
}

\author{
Een andere kijk op de levensloop \\ van granulocyten in de mens
}

(met een samenvatting in het Nederlands)

\section{Proefschrift}

ter verkrijging van de graad van doctor aan de Universiteit Utrecht op gezag van de rector magnificus, prof.dr. H.R.B.M. Kummeling, ingevolge het besluit van het college voor promoties in het openbaar te verdedigen op maandag 14 september 2020 des middags te 12.45 uur

door

\section{Marwan Hassani}

geboren op 3 oktober 1988

te Baghdad, Irak 


\section{Promotor:}

Prof. dr. L. Koenderman 
Aan mijn ouders 



\section{Contents}

Chapter 1

General introduction

Part I: Neutrophil Kinetics

Chapter 2

Granulocyte kinetics are best described in a linear

25

conveyor belt model

Chapter 3

On the origin of low-density neutrophils

41

Chapter 4

Mepolizumab does not affect neutrophil

kintetics in eosinophilic asthma

Chapter 5

Unique cross-talk between GM-CSF and

CXCR4 signaling in human neutrophils

Chapter 6

Chapter 7

Chapter 8

Chapter 9

Chapter 10

Chapter 11

Appendix

Nederlandse Samenvatting

Immunological and hematological effects of

IL-5(R $\alpha)$ targeted therapy: an overview

Characterization of the phenotype of human eosinophils and their progenitors in the bone marrow of healthy individuals

Differentiation and activation of eosinophils in the human bone marrow during experimental human endotoxemia

The effect of mepolizumab on eosinophil and basophil kinetics

in patients with eosinophilic asthma

The effect of dupilumab on eosinophil homing and activation in patients with atopic dermatitis

General discussion

Dankwoord

Curriculum Vitae 


\section{Chapter 1}

General introduction 


\section{Neutrophil development and life cycle}

Unlike eosinophils, the role of neutrophils in immunity is relatively well known. Their importance is underlined by the fact that neutrophil-like cells are present in even the most "simple" lifeforms that lack a body cavity or a vascular system ${ }^{1}$. In humans, neutrophils are involved in early recognition and killing of infectious pathogens. This is underlined by the fact that severe congenital neutropenias, a heterogeneous group of rare hematological disorders, leads to recurrent and life-threatening (bacterial) infections $s^{2}$ In contrast, hyper-activation and/or induction of sterile neutrophilia can cause damage. These harmful effects of neutrophils are for example seen in cases of $\mathrm{ARDS}^{3}$. So, a controlled neutrophil production, release from the bone marrow and clearance mechanism are important to protect the host against inflammatory damage on the one hand or infections on the other.

The production of neutrophils mainly takes place in the bone marrow. The coordinated expression of certain transcription factors, such as PU.1, CBF, C-Myb and C/EBPs regulate the differentiation of hematopoietic stem cells (HSCs) into the myeloid lineage ${ }^{4}$. The first inscribed neutrophil progeny is the myeloblast which consecutively differentiates and matures into promyelocyte, myelocyte, metamyelocyte, neutrophils with a banded nucleus ('banded neutrophils') and finally into a neutrophil with a segmented nucleus ("segmented neutrophils" see Figure 1$)^{5}$.

During the maturation of neutrophils, different granules (azurophilic, specific and gelatinase containing granules) are formed (Figure 1). Finally, after the formation of granules, the secretory vesicles are formed ${ }^{6}$. Each of these granules and vesicles are packed with different proteins that contribute to bacterial killing when they are released inside the phagosome or extracellularly. Certain granule membrane proteins, e.g. CD66b in specific granules (Figure 1), are expressed on the cell surface after fusion of the granules with the membrane induced by neutrophil activation. This fusion then leads to release the granule proteins into the extracellular environment in a process referred to as degranulation. Only a mild stimulus is needed to secrete the content of secretory vesicles. In contrast, increasing activation is necessary for degranulation of the different granules: gelatinase containing granules first, followed by specific and finally azurophilic granules ${ }^{7}$. Remarkably, this order of degranulation is the exact opposite of the order of formation of granules during the maturation process of neutrophils.

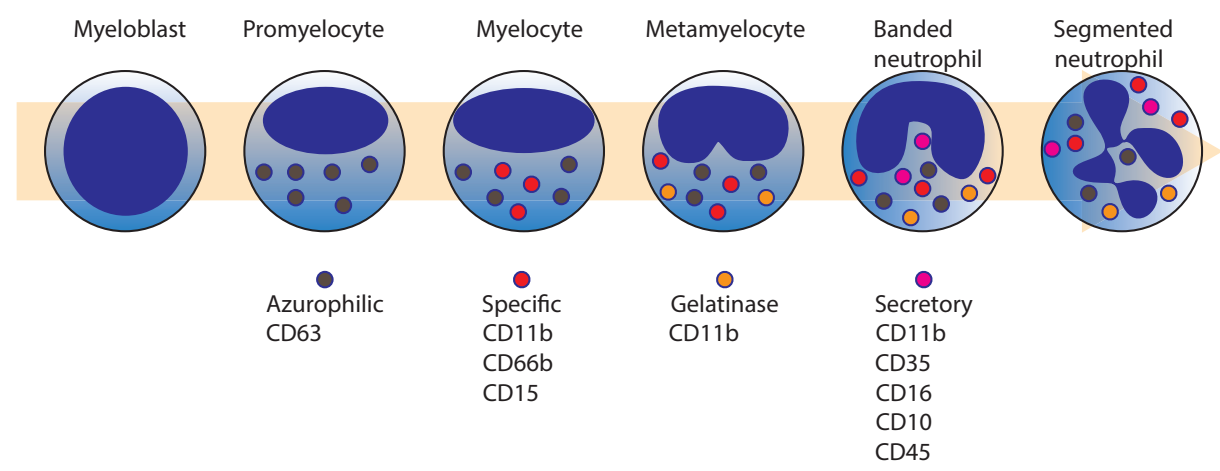

Figure 1. Schematic overview of granulopoiesis and the formation of different granules. 
During granulopoiesis: myeloblasts, promyelocytes and myelocytes are able to proliferate. Metamyelocytes lose the ability to go into mitosis. Therefore from this stage on, the progenitors enter the post mitotic pool ${ }^{8}$. The post mitotic pool transit time (PMPtt) takes around 6 days for neutrophils (chapter 2 and 9) and 4 days for eosinophils (chapter 9) $9,10,11$.

After maturation, segmented neutrophils are released into the bloodstream. Since the number of tissue neutrophils is very low during homeostasis, it is not likely that neutrophils extravasate without an inflammatory (infectious or non-infectious) cue ${ }^{12,13}$. This is however, still a subject of debate, because in murine studies neutrophils are found to infiltrate healthy intestines. This infiltration has a circadian pattern which is believed to serve as a signal for distant bone marrow niches to regulate neutrophil production ${ }^{14}$.

Moreover, similar to eosinophils the half-life of neutrophils has not been established consistently. The mentioned half-life of (circulatory) neutrophils in the literature differs between 4.2 and 90 hours $^{8}$. The probable reason for this variation is because old studies suffer from technical limitations, such as the use of toxic (radioactive) labels or in vitro manipulations of cells ${ }^{8}$. Both can influence neutrophil survival and homing characteristics. In newer studies in which a non-toxic label was used (deuterium), the determination of neutrophil half-life still proofed to be a great challenge. Deuterium labeled DNA from circulatory neutrophils can be found in the blood as long as 15 days after ingestion of label ${ }^{11}$. This data is however impossible to interpret without a reliable model to distinguish between labeled cells residing in the blood and those that are released with a delay by the bone marrow ${ }^{11,15}$

On the other hand, there are still uncertainties about how and where neutrophils are cleared from the body regardless the duration of the half-life. At least it is apparent that under homeostatic conditions, clearance of the neutrophils must match the production in the bone marrow. It is believed that this clearance occurs in the liver, the marginal zone of the spleen and in the bone marrow stroma ${ }^{16}$.

\section{The role of CXCR4 in neutrophil kinetics}

Recruited neutrophils to the tissues during inflammation are mainly cleared by macrophages in situ, but some cells can also be removed by dendritic cells, or by exiting the site of inflammation through draining lymph ${ }^{17}$. In addition to apoptotic neutrophils, there are also neutrophils that signal for removal before they get truly apoptotic ${ }^{18}$. Due to the large reserve of mature neutrophils in the bone marrow, and the rapid mobilization of neutrophils during inflammation, it is suggested that specific molecular mechanisms regulate the homing of the neutrophils back to the bone marrow. A brief activation of NADPH oxidase in neutrophils enhances the removal of neutrophils both in vitro and in vivo by generating the expression of surface ligands similar to those of apoptotic neutrophils ${ }^{18}$. Aged neutrophils may also generate these pre-apoptotic signals. One of these signals is expression of the chemokine receptor CXCR4, which plays an important role in the neutrophil release from the bone marrow ${ }^{19}$. Enhanced expression of CXCR4 on aged neutrophils is hypothesized to result in the homing of aged neutrophils to the bone marrow. While the CXCR4 expression is upregulated, the expression of chemotaxis receptor CXCR2 is decreased ${ }^{20}$. Thus, chemokine signaling between the receptors CXCR4 and CXCR2 and their ligands may play an important role in the clearance of aged 
neutrophils, but more evidence is needed to understand the mechanism behind this process.

CXCL12 or stromal derived factor-1 $\alpha$ (SDF-1 $\alpha$ ) functions as the ligand of CXCR4, but can also bind to CXCR7 ${ }^{21}$. Such promiscuity is not unique for CXCL12, as multiple chemokines can bind to different receptors, and most chemokine receptors have multiple ligands ${ }^{22}$. The N-terminal residues of CXCL12 bind to the extracellular groove formed by the helices of CXCR4, resulting in a conformational change of the transmembrane parts of the receptor $^{23}$. This change allows intracellular interaction between the intracellular domain of CXCR4 with the coupled G protein. This activation triggers several signal cascades, among which PI3K/AKT/mTOR and ERK1/2 are the most important ones ${ }^{23}$. Both pathways play an important role in cell survival and proliferation ${ }^{24}$. One of the other possible signaling cascades stimulated by activation of the G-protein is the activation of phospholipase C. This activation is preceded by the dissociation of the $\beta$ and $\gamma$ subunits of the GPCR (Figure 2), which in turn breaks down the phosphoinositol lipid PIP2 into IP3 and diacylglycerol (DAG). IP3 functions as second messenger that binds to the IP3 receptor present on the intracellular $\mathrm{Ca}^{2+}$ reservoirs (Figure 2). This interaction leads to the release of free $\mathrm{Ca}^{2+}$ in the cytosol ${ }^{25}$. This spatio-temporal regulation of cytosolic $\mathrm{Ca}^{2+}$ adjusts the migration machinery, actin polarization and focal adhesion dynamics of the cell ${ }^{26}$.

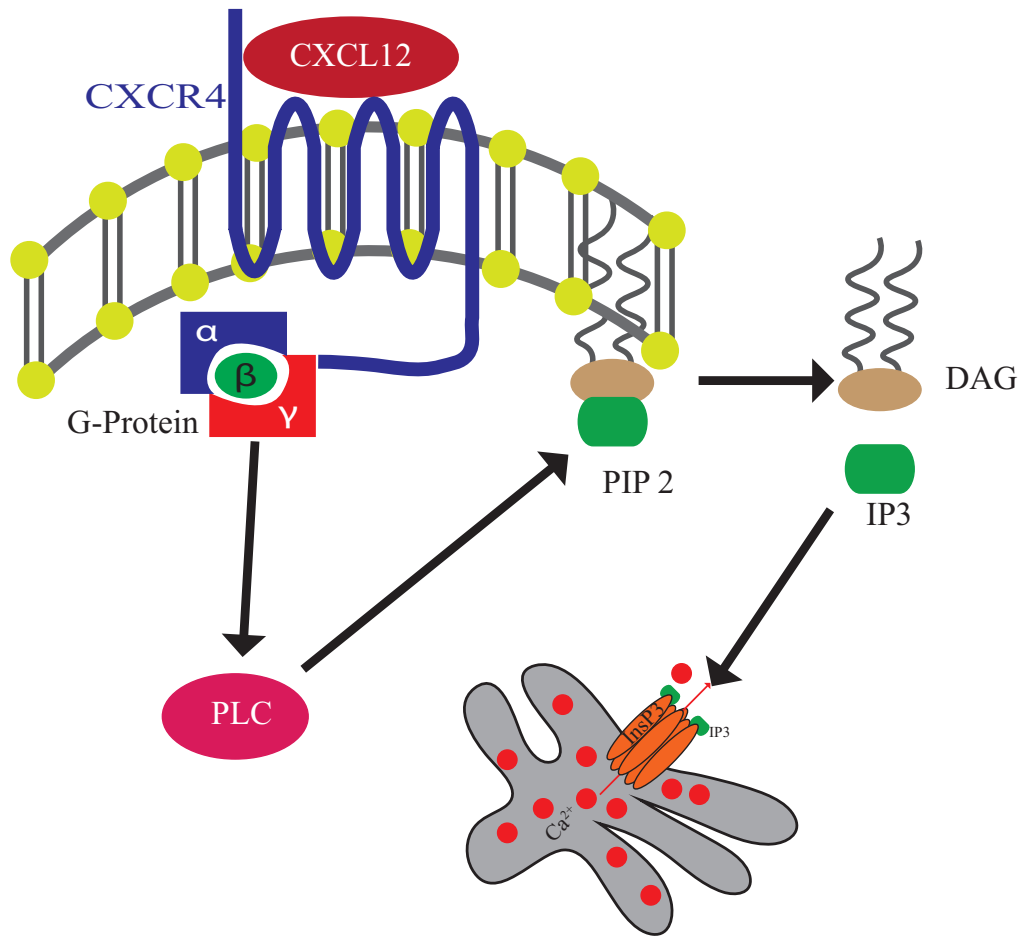

Figure 2. Schematic overview of mobilization of $\mathrm{Ca}^{2+}$ from internal stores through CXCR4 signaling.

After CXCL12 binds to CXCR4, there is a conformational change of the transmembrane helices of CXCR4 which allows the intracellular domain of this receptor to interact with $\mathrm{G}$ protein-coupled 
receptor (GPCR). This leads to the activation of phosopholipase C- $\beta$ (PLC- $\beta$ ). Activated PLC breaks down Phosphatidylinositol 4,5-bisphosphate (PIP2) into diacylglycerol (DAG) and Inositol trisphosphate (IP3). IP3 binds to the IP3 sensitive Ca2+ channels (InsP3 receptor) on the endoplasmatic reticulum (ER) which leads to the mobilization of $\mathrm{Ca} 2+$ from the ER into the cytosol.

\section{Eosinophil development and life cycle}

Eosinophils were discovered in 1879 by Paul Ehrlich ${ }^{27,28}$. Since then these cells have been extensively studied by both clinicians and scientists. The still growing interest in eosinophils is quite remarkable, because unlike other leukocytes their removal or depletion does not seem to lead to a clear deleterious effect in living mammals ${ }^{29}$. On the other hand, humans without eosinophils are extremely rare indicating a role of these cells in homeostasis.

In homeostasis eosinophils are mainly produced in the bone marrow (chapter 7). Three cytokines, IL-3, IL-5 and GM-CSF are important in regulating their development ${ }^{30}$. These three "eosinophilopoietins" have different receptors containing cytokine specific $\alpha$-chains linked to a common $\beta$-chain ${ }^{31}$. Although the effect of these receptors is mainly orchestrated via the $\beta$-chain, we have shown IL-5 $\alpha$-chain specific signaling ${ }^{32}$. The human eosinophil expresses all three receptors ${ }^{33}$. IL-5 is the most eosinophil specific cytokine as the receptor is only shared with basophils. This cytokine is not only important for the proliferation (and differentiation) of eosinophils but also regulates eosinophil survival and mobilization ${ }^{34}$ (chapter 6).

After differentiation and maturation eosinophils are released into the circulation. In the bloodstream they compromise around 1-3\% of all leukocytes ${ }^{35}$. From the blood they mostly traffic into the gastro-intestinal tract, where they normally reside within the lamina propria of all compartments except for the esophagus ${ }^{36}$. The trafficking of eosinophils is regulated by eotaxins. The specific activity of all eotaxins (1,2 and 3 ) is mediated by the selective expression of the transmembrane, $\mathrm{G}$ protein-coupled receptor CCR3, primarily expressed on eosinophils ${ }^{37}$. Eotaxins and IL-5 have an interplay and together they play an important role in regulating tissue eosinophila ${ }^{38}$.

In the fully developed stage, eosinophils have multiple (specific) granules. These granules contain numerous enzymatic and nonenzymatic cationic proteins, such as major basic protein 1 (MBP-1), eosinophil peroxidase (EPX) and eosinophilic cationic protein $(\mathrm{ECP})^{39}$. With these cytotoxic granule proteins they are capable to defend the host against invading multicellular parasites, particularly helminths ${ }^{40}$. This anti-parasitic effect has not been fully elucidated, but sufficient evidence has been provided showing that these cells are capable to attack and kill helminths with a size much larger than the cells. They do this by "together we are strong" type of mechanism by which a multitude of cells kill the large parasite together ${ }^{41}$. The killing mechanisms are illustrated by the ability to mediate antibody dependent cellular toxicity against helminths in vitro. Additional evidence for the importance of parasite killing by eosinophils comes from the observation that eosinophil numbers increase during numerous parasitic infections in humans and that their granular products were find in the vicinity of damaged parasites in vivo ${ }^{42}$. The complexity of the interaction between eosinophils and parasites is underlined by the fact that these cells can also increase survival of certain parasites ${ }^{43}$. 


\section{Homeostatic Eosinophils: Characteristics and Functions}

At steady state, eosinophils are not only distributed in the blood but also in the thymus, uterus, adipose tissues, mammary gland, spleen and the gastro-intestinal tract, indicating a physiological function in each organ ${ }^{44}$. It should be emphasized that most information about these homeostatic eosinophils was collected from murine studies ${ }^{45}$. In these studies, it was displayed that homeostatic eosinophils shared identical morphology with inflammatory eosinophils. Dependent on the type of tissue in which they reside, they are believed to have diverse functional characteristics ${ }^{45}$. In the gastrointestinal tract, they are most likely involved in the regulation and defense against the constant and extreme exposition to potentially pathogenic microorganisms and foreign (including certain food) antigens ${ }^{46}$.

In the adipose tissue, eosinophils are the main source of IL-4 production which is important in the polarization of adipose macrophages toward the alternatively activated phenotype. These alternatively activated macrophages play a crucial role in glucose homeostasis and development of beige fat. Without eosinophils biogenesis of beige fat is impaired, at least in mice ${ }^{47}$.

Thymic eosinophils are believed to be involved in negative T-cell selection, again in mice $^{48}$. Whereas, murine eosinophils in the mammary gland were shown to be important in regulating postnatal mammary gland development ${ }^{49}$.

So, it is apparent that besides their cytotoxic functions, eosinophils have multiple immune and non-immune regulatory functions ${ }^{50}$. Remarkably, what happens after eosinophils have homed to the tissue has not been extensively studied. For example, the lifespan of eosinophils is still a subject of debate. Different studies document different eosinophil lifespans (with a large variety between 11 and 63 hours) ${ }^{51,9,52}$. Even less is known about how eosinophils are cleared from the tissue and circulation. It has been suggested that apoptotic eosinophils are cleared from the tissue by macrophages and epithelial cells by a process called efferocytosis ${ }^{53}$. It is clear that more research is needed to determine a reliable eosinophil lifespan. This has been attempted in this thesis (chapter 9).

\section{The role of eosinophils in asthma}

Eosinophils do not only have a beneficial role as is described above but also have been long associated with the pathology of multiple diseases, one of which is asthma. A first hint towards a pathological role in asthma was already made in 1922 by Huber and Koessler who found massive blood and tissue eosinophilia in the lungs of patients dying of asthma ${ }^{54}$. The years that followed eosinophils were mainly considered to play a protective role in asthma. It was believed that the eosinophil is attracted at the level of the bronchus to enzymatically neutralize the harmful mediators released by the mast cells (histamine, leukotriene $C_{4}$ and leukotriene $D_{4}$ ) ${ }^{55}$. The fact that the number of eosinophils in the circulation strongly correlated with asthma severity did not exclude this beneficial effect ${ }^{56}$. However this paradigm changed quickly when it was discovered that elevated levels of eosinophil granule proteins, such as MBP were also found in the tissue and broncheoalveolar lavage (BAL) fluid of asthma patients ${ }^{57}$. These levels are sufficient to cause damage to respiratory epithelial cells in vitro and trigger degranulation of mast cells and basophils ${ }^{44}$. Moreover MBP can directly bind 
to muscarine receptors (M2) in the bronchial airways and cause smooth muscle cells contraction, which is also an important feature of asthma. ${ }^{58}$ Besides, eosinophils generate great amounts of leukotriene $C_{4}$ themselves, which is metabolized to leukotriene $\mathrm{D}_{4}$ and leukotriene $\mathrm{E}_{4}$. These three lipid mediators are part of the so-called slow reacting substances of anaphylaxis (SRS-A). They are known to increase vascular permeability and mucus secretion and are also a potent stimulators of smooth-muscle contraction ${ }^{59}$.

Nowadays the aim of asthma treatment is to reduce the number of eosinophils in the blood and sputum. Steroid treatment with the purpose to reduce sputum eosinophil numbers in asthma patients indeed lowered the number of exacerbations ${ }^{60}$. Since steroids have multiple harmful side-effects, especially when they are used for a longer period of time ${ }^{61}$, specific anti-eosinophil therapy has been developed. Targeting IL-5 strongly reduces the number of eosinophils in blood and sputum with the subsequent reduction of asthma exacerbations and tapering of corticosteroids (chapter 6$)^{62}$.

\section{The role of eosinophils in other (atopic) disorders}

Eosinophils are linked to several other pathological conditions. These conditions are also accompanied by a (large) increase in eosinophil infiltration of certain organs with subsequent tissue damage. For example, pathological infiltration of eosinophils in the esophagus, stomach, small intestine or colon, as is seen in eosinophilic gastrointestinal disorders, can lead to organ dysfunction and clinical symptoms ${ }^{63}$.

In atopic dermatitis (AD), the role of eosinophils is uncertain. There is evidence suggesting their involvement, such as the finding of eosinophil toxic granular proteins in the skin of patients with $A D^{64}$. However short term treatment with mepolizumab did not improve symptoms of patients with $A D^{65}$. Treatment with dupilumab (an IL-4R $\alpha$ antagonist) does lead to improvement in symptoms in AD patients despite circulatory eosinophilia ${ }^{66}$. It is however possible that eosinophils are not able to home to the skin as a result of IL-4 and IL-13 inhibition (chapter 10) ${ }^{67}$.

In contrast, acute bacterial infection leads to an opposite phenomenon: instead of eosinophilia, a marked decrease in circulating eosinophils (eosinopenia) is seen ${ }^{68}$. Relatively little is known about this process, other than that it is not only associated with an increase in endogenous glucocorticoids ${ }^{69}$. An alternative explanation for the observed eosinopenia is an increase of homing of eosinophils to the tissues as is described in chapter 8.

\section{Scope and outline of this thesis}

The aim of this thesis is to better understand the kinetics of granulocytes in health and during type 2 inflammation.

Part 1 of this thesis focuses on the kinetics of neutrophils. In chapter $\mathbf{2}$ we studied the kinetics of neutrophils and their progenitors by following the deuterium label over time in bone marrow granulocytes, sampled from healthy human volunteers. In order to reliably interpret labelling data of circulatory granulocytes. We also explore which model (ODE versus linear conveyor belt model) best explains the data. In chapter 3 we review the hypothesis that buoyant density of neutrophils is characterized by a spectrum which as a whole shift to a lower density after activation. In blood from 
healthy donors, neutrophils can be separated from peripheral blood mononuclear cells (PBMCs) by using density centrifugation. In this setting neutrophils are not found in the PBMC fraction, because their buoyant density is higher than $1.077 \mathrm{~g} / \mathrm{ml}$. However, during several pro-inflammatory conditions, such as cancer, SLE or asthma neutrophils are co-segregated with $\mathrm{PBMCs}^{70}$. Because these cells show different functional properties (both pro-inflammatory and anti-inflammatory) in different conditions, it is important to understand their origin (activation of "normal neutrophils" versus separate lineage). Chapter 4 describes the kinetics of normal mature neutrophils (CD16 ${ }^{\text {high }} \mathrm{CD} 2 \mathrm{~L}^{\text {high }}$ ) hypersegmented neutrophils (CD16 ${ }^{\text {high }} \mathrm{CD} 2 \mathrm{~L}^{\mathrm{dim}}$ ) and neutrophil progenitors (CD16 neg $C D 62 L^{\text {neg }}$ ) in the blood (and for mature neutrophils also sputum), again by using deuterated glucose in asthma patients that were either treated with mepolizumab or placebo. In this study, deuterated glucose was used to label the cells of interest. This method is a reliable way to provide information about cellular kinetics, because it is non-toxic and labeling occurs in vivo ${ }^{71}$. In chapter 5 we show that freshly isolated neutrophils from healthy volunteers have a functional CXCR4 receptor despite a very low receptor expression on the membrane. Moreover, we show that $\mathrm{Ca}^{2+}$ influx, caused by activation of this receptor through binding of CXCL12, can be completely blocked after treatment with GM-CSF.

The second part of this thesis focuses on the kinetics of eosinophils. Chapter 6 presents an overview of the effect of IL-5 targeted therapy on immune cells, but more specifically on the eosinophil lineage. IL-5 is an essential cytokine for eosinophil proliferation, differentiation and survival. Chapter 7 provides a novel flow cytometric method to identify eosinophil (middle late) progenitors in the bone marrow. Moreover, it shows how certain surface markers change during eosinophil maturation in the bone marrow. In chapter $\mathbf{8}$, a hypothesis is provided on how acute (bacterial) inflammation can lead to circulatory eosinopenia. This hypothesis is supported by blood and bone marrow data after experimental endotoxemia, which is accomplished by systemic administration of $2 \mathrm{ng} / \mathrm{kg}$ LPS in healthy human volunteers. Chapter 9 describes eosinophil and basophil kinetics in patients with eosinophilic asthma that were randomized for treatment with mepolizumab or placebo. Chapter 10 shows a possible mechanism on how eosinophila arises after treatment with anti-IL-4R (dupilumab) in patients with atopic dermatitis. Furthermore, this chapter shows whether eosinophils in atopic dermatitis show signs of activation compared to healthy donors.

Finally, in chapter 11 the results of the previous chapters are summarized and discussed. 


\section{References}

1. Leiding JW. Neutrophil Evolution and Their Diseases in Humans. Front Immunol. 2017;8:1009.

2. Skokowa J, Dale DC, Touw IP, et al. Severe congenital neutropenias. Nat Rev Dis Prim. 2017;3:17032.

3. Rebetz J, Semple JW, Kapur R. The Pathogenic Involvement of Neutrophils in Acute Respiratory Distress Syndrome and Transfusion-Related Acute Lung Injury. Transfus Med Hemotherapy. 2018;45:290-298.

4. Rosmarin AG, Yang Z, Resendes KK. Transcriptional regulation in myelopoiesis: Hematopoietic fate choice, myeloid differentiation, and leukemogenesis. Exp Hematol. 2005;33:131-143.

5. Lawrence SM, Corriden R, Nizet V. The Ontogeny of a Neutrophil: Mechanisms of Granulopoiesis and Homeostasis. Microbiol Mol Biol Rev;82 . Epub ahead of print 2018. DOI: 10.1128/MMBR.00057-17.

6. Cowland JB, Borregaard N. Granulopoiesis and granules of human neutrophils. Immunol Rev. 2016;273:11-28.

7. Gullberg U, Bengtsson N, Bülow E, et al. Processing and targeting of granule proteins in human neutrophils. J Immunol Methods. 1999;232:201-10.

8. Tak T, Tesselaar K, Pillay J, et al. What's your age again? Determination of human neutrophil half-lives revisited. J Leukoc Biol. 2013;94:595-601.

9. Steinbach $\mathrm{KH}$, Schick P, Trepel F, et al. Estimation of kinetic parameters of neutrophilic, eosinophilic, and basophilic granulocytes in human blood. Blut. 1979;39:27-38.

10. Pillay J, den Braber I, Vrisekoop N, et al. In vivo labeling with $2 \mathrm{H} 2 \mathrm{O}$ reveals a human neutrophil lifespan of 5.4 days. Blood. 2010;116:625-7.

11. Lahoz-Beneytez J, Elemans $M$, Zhang $\mathrm{Y}$, et al. Human neutrophil kinetics: modeling of stable isotope labeling data supports short blood neutrophil half-lives. Blood. 2016;127:34313438.

12. Hidalgo A, Chilvers ER, Summers C, et al. The Neutrophil Life Cycle. Trends Immunol. 2019;40:584-597.

13. PETERS AM, RODDIE ME, DANPURE HJ, et al. 99Tcm-HMPAO labelled leucocytes. Nucl Med Commun. 1988;9:449-464.

14. Casanova-Acebes M, Nicolás-Ávila JA, Li JL, et al. Neutrophils instruct homeostatic and pathological states in naive tissues. J Exp Med. 2018;215:2778-2795.

15. Ahmed R, Westera L, Drylewicz J, et al. Reconciling Estimates of Cell Proliferation from Stable Isotope Labeling Experiments. PLOS Comput Biol. 2015;11:e1004355.

16. Gordy C, Pua H, Sempowski GD, et al. Regulation of steady-state neutrophil homeostasis by macrophages. Blood. 2011;117:618-629.

17. Henson PM, Hume DA. Apoptotic cell removal in development and tissue homeostasis. Trends Immunol. 2006;27:244-250.

18. Frasch SC, Berry KZ, Fernandez-Boyanapalli R, et al. NADPH Oxidase-dependent Generation of Lysophosphatidylserine Enhances Clearance of Activated and Dying Neutrophils via G2A. J Biol Chem. 2008;283:33736-33749.

19. De Filippo K, Rankin SM. CXCR4, the master regulator of neutrophil trafficking in homeostasis and disease. Eur J Clin Invest. 2018;48:e12949.

20. Martin C, Burdon PCE, Bridger G, et al. Chemokines acting via CXCR2 and CXCR4 control the release of neutrophils from the bone marrow and their return following senescence. Immunity. 2003;19:583-93. 
21. Ma D-M, Luo D-X, Zhang J. SDF-1/CXCR7 axis regulates the proliferation, invasion, adhesion, and angiogenesis of gastric cancer cells. World J Surg Oncol. 2016;14:256.

22. Stone MJ, Hayward JA, Huang C, et al. Mechanisms of Regulation of the ChemokineReceptor Network. Int J Mol Sci;18 . Epub ahead of print February 7, 2017. DOI: 10.3390/ ijms18020342.

23. Crump MP, Gong JH, Loetscher P, et al. Solution structure and basis for functional activity of stromal cell-derived factor-1; dissociation of CXCR4 activation from binding and inhibition of HIV-1. EMBO J. 1997;16:6996-7007.

24. Roland J, Murphy BJ, Ahr B, et al. Role of the intracellular domains of CXCR4 in SDF-1mediated signaling. Blood. 2003;101:399-406.

25. Putney JW, Tomita T. Phospholipase C signaling and calcium influx. Adv Biol Regul. 2012;52:152-164.

26. Paupe V, Prudent J. New insights into the role of mitochondrial calcium homeostasis in cell migration. Biochem Biophys Res Commun. 2018;500:75-86.

27. Ehrlich P. Beiträge zur Kenntniss der granulirten Bindegewebszellen und der eosinophilen Leukocythen. Arch Anat Physiol;166 . Epub ahead of print 1879. DOI: 10.1016/b978-0-08009054-2.50011-0.

28. Kay AB. The early history of the eosinophil. Clin Exp Allergy. 2015;45:575-582.

29. Gleich GJ, Klion AD, Lee JJ, et al. The consequences of not having eosinophils. Allergy. 2013;68:829-35.

30. Hassani M, Koenderman L. Immunological and hematological effects of IL-5(R $\alpha)$-targeted therapy: An overview. Allergy. 2018;73:1979-1988.

31. Takatsu K. Interleukin-5 and IL-5 receptor in health and diseases. Proc Jpn Acad Ser B Phys Biol Sci. 2011;87:463-485.

32. Geijsen N, Uings IJ, Pals C, et al. Cytokine-specific transcriptional regulation through an IL5Ralpha interacting protein. Science. 2001;293:1136-8.

33. Kalinauskaite-Zukauske $V$, Januskevicius A, Janulaityte I, et al. Expression of eosinophil $\beta$ chain-signaling cytokines receptors, outer-membrane integrins, and type 2 inflammation biomarkers in severe non-allergic eosinophilic asthma. BMC Pulm Med. 2019;19:158.

34. Sanderson CJ. Interleukin-5, eosinophils, and disease. Blood. 1992;79:3101-3109.

35. Uhm TG, Kim BS, Chung IY. Eosinophil Development, Regulation of Eosinophil-Specific Genes, and Role of Eosinophils in the Pathogenesis of Asthma. Allergy, Asthma Immunol Res. 2012;4:68.

36. Mishra A, Hogan SP, Lee JJ, et al. Fundamental signals that regulate eosinophil homing to the gastrointestinal tract. J Clin Invest. 1999;103:1719-1727.

37. Murphy PM. The Molecular Biology of Leukocyte Chemoattractant Receptors. Annu Rev Immunol. 1994;12:593-633.

38. Rosenwasser LJ, Zimmermann N, Hershey GK, et al. Chemokines in asthma: Cooperative interaction between chemokines and IL-13. J Allergy Clin Immunol. 2003;111:227-242.

39. Acharya KR, Ackerman SJ. Eosinophil granule proteins: form and function. J Biol Chem. 2014;289:17406-17415.

40. Klion AD, Nutman TB. The role of eosinophils in host defense against helminth parasites. J Allergy Clin Immunol. 2004;113:30-37.

41. Patnode ML, Bando JK, Krummel MF, et al. Leukotriene B4 amplifies eosinophil accumulation in response to nematodes. J Exp Med. 2014;211:1281-8. 
42. Butterworth AE. The Eosinophil and its Role in Immunity to Helminth Infection. In: Current topics in microbiology and immunology:127-168.

43. Gebreselassie NG, Moorhead AR, Fabre V, et al. Eosinophils Preserve Parasitic Nematode Larvae by Regulating Local Immunity. J Immunol. 2012;188:417-425.

44. Rothenberg ME, Hogan SP. THE EOSINOPHIL. Annu Rev Immunol. 2006;24:147-174.

45. Marichal T, Mesnil C, Bureau F. Homeostatic Eosinophils: Characteristics and Functions. Front Med. 2017;4:101.

46. Chu VT, Beller A, Rausch S, et al. Eosinophils promote generation and maintenance of immunoglobulin-A-expressing plasma cells and contribute to gut immune homeostasis. Immunity. 2014;40:582-93.

47. Wu D, Molofsky AB, Liang H-E, et al. Eosinophils Sustain Adipose Alternatively Activated Macrophages Associated with Glucose Homeostasis. Science (80- ). 2011;332:243-247.

48. Throsby $M$, Herbelin A, Pléau JM, et al. CD11C+ eosinophils in the murine thymus: developmental regulation and recruitment upon MHC class I-restricted thymocyte deletion. J Immunol. 2000;165:1965-75.

49. Gouon-Evans V, Lin EY, Pollard JW. Requirement of macrophages and eosinophils and their cytokines/chemokines for mammary gland development. Breast Cancer Res. 2002;4:15564.

50. Wen T, Rothenberg ME. The Regulatory Function of Eosinophils. Microbiol Spectr;4. Epub ahead of print 2016. DOI: 10.1128/microbiolspec.MCHD-0020-2015.

51. Farahi N, Singh NR, Heard S, et al. Use of 111-Indium-labeled autologous eosinophils to establish the in vivo kinetics of human eosinophils in healthy subjects. Blood. 2012;120:40684071.

52. Parwaresch MR, Walle AJ, Arndt D. The peripheral kinetics of human radiolabelled eosinophils. Virchows Arch B Cell Pathol. 1976;57-66.

53. Felton JM, Lucas $C D$, Rossi AG, et al. Eosinophils in the lung - modulating apoptosis and efferocytosis in airway inflammation. Front Immunol. 2014;5:302.

54. Huber HL; Koessler KK. The pathology of bronchial asthma. Arch intern med. 1922;30:689.

55. FRIGAS E, GLEICH G. The eosinophil and the pathophysiology of asthma. J Allergy Clin Immunol. 1986;77:527-537.

56. Horn BR, Robin ED, Theodore J, et al. Total Eosinophil Counts in the Management of Bronchial Asthma. N Engl J Med. 1975;292:1152-1155.

57. Rothenberg ME. Eosinophilia. N Engl J Med. 1998;338:1592-1600.

58. Jacoby DB, Gleich GJ, Fryer AD. Human eosinophil major basic protein is an endogenous allosteric antagonist at the inhibitory muscarinic M2 receptor. J Clin Invest. 1993;91:13141318.

59. Epstein FH, Lewis RA, Austen KF, et al. Leukotrienes and Other Products of the 5-Lipoxygenase Pathway. N Engl J Med. 1990;323:645-655.

60. Green RH, Brightling CE, McKenna S, et al. Asthma exacerbations and sputum eosinophil counts: a randomised controlled trial. Lancet. 2002;360:1715-1721.

61. Oray M, Abu Samra K, Ebrahimiadib N, et al. Long-term side effects of glucocorticoids. Expert Opin Drug Saf. 2016;15:457-465.

62. Farne HA, Wilson A, Powell C, et al. Anti-IL5 therapies for asthma. Cochrane Database Syst Rev. 2017;9:CD010834.

63. Rothenberg ME. Eosinophilic gastrointestinal disorders (EGID)拧. J Allergy Clin Immunol. 2004;113:11-28. 
64. Kiehl P, Falkenberg K, Vogelbruch M, et al. Tissue eosinophilia in acute and chronic atopic dermatitis: a morphometric approach using quantitative image analysis of immunostaining. Br J Dermatol. 2001;145:720-9.

65. Oldhoff JM, Darsow U, Werfel T, et al. Anti-IL-5 recombinant humanized monoclonal antibody (Mepolizumab) for the treatment of atopic dermatitis. Allergy. 2005;60:693-696.

66. Simpson EL, Bieber T, Guttman-Yassky E, et al. Two Phase 3 Trials of Dupilumab versus Placebo in Atopic Dermatitis. N Engl J Med. 2016;375:2335-2348.

67. Jonstam K, Swanson BN, Mannent LP, et al. Dupilumab reduces local type 2 pro-inflammatory biomarkers in chronic rhinosinusitis with nasal polyposis. Allergy. 2019;74:743-752.

68. Abidi K, Khoudri I, Belayachi J, et al. Eosinopenia is a reliable marker of sepsis on admission to medical intensive care units. Crit Care. 2008;12:R59.

69. Bass DA. Behavior of eosinophil leukocytes in acute inflammation. I. Lack of dependence on adrenal function. J Clin Invest. 1975;55:1229-1236.

70. Scapini P, Marini O, Tecchio C, et al. Human neutrophils in the saga of cellular heterogeneity: insights and open questions. Immunol Rev. 2016;273:48-60.

71. Macallan DC, Asquith B, Zhang $\mathrm{Y}$, et al. Measurement of proliferation and disappearance of rapid turnover cell populations in human studies using deuterium-labeled glucose. Nat Protoc. 2009;4:1313-27. 



\section{Part I}

Neutrophil kinetics 


\section{Chapter 2}

\section{Granulocyte kinetics are best described in a linear conveyor belt model}

Marwan Hassani ${ }^{1}$, Erinke van Grinsven ${ }^{1}$, Tamar Tak ${ }^{1}$, Selma van Staveren ${ }^{1}$, Guus Leijte ${ }^{2}$, Niklas Bruse ${ }^{2}$, Matthijs Kox ${ }^{2}$, Lucie Hustin ${ }^{1}$, Corneli van Aalst ${ }^{1}$, José Borghans ${ }^{3}$, Julia Drylewicz ${ }^{3}$, Rob de Boer ${ }^{3}$, Peter Pickkers', Kiki Tesselaar ${ }^{3}$, Nienke Vrisekoop ${ }^{1}$, Leo Koenderman ${ }^{1}$

1. Department of Respiratory Medicine and Center for Translational Immunology, University Medical Center Utrecht, The Netherlands.

2. Department of Intensive Care and Radboud Center for Infectious Diseases (RCI), Radboud University Medical Center, Nijmegen, The Netherlands.

3. Center for Translational Immunology, University Medical Centre Utrecht, Utrecht, The Netherlands. 


\section{Abstract}

In recent years, new methods to label circulatory neutrophils in vivo have become available and have sparked a fierce debate on the circulatory lifespan of the human neutrophil. Advantages of the new method of in vivo labelling with the stable isotope ${ }^{2} \mathrm{H}$ (deuterium) are the lack of toxicity and no requirement for ex vivo manipulation. However, a disadvantage is the delay between incorporation of the label in the DNA of dividing progenitors in the bone marrow and the measurement of label in the DNA of mature neutrophils in the peripheral blood. The gap in knowledge on neutrophil progenitor proliferation and differentiation in the bone marrow precluded adequate analysis of the kinetics of circulatory neutrophils. Therefore, there is no consensus on the circulatory lifespan, with estimates ranging from 6 hours to 5.4 days.

The current study is the first study to measure the kinetics of mature neutrophils and their different neutrophil progenitors during granulopoiesis in the human bone marrow and blood in healthy volunteers.

$6,6-{ }^{2} \mathrm{H}$-glucose ( $70 \mathrm{~g} /$ individual) pulse-chase labelling was applied in 12 healthy volunteers, and at 1 to 9 days after the labelling, blood and bone marrow samples were simultaneously obtained. With a new flow cytometry strategy, the different progenitor stadia of the neutrophil were sorted and for each the DNA was isolated. The ${ }^{2} \mathrm{H}$-label enrichment in the DNA was determined by a combination of gas chromatography and mass spectrometry.

The kinetics of ${ }^{2} \mathrm{H}$-enrichment over time showed a delay of 2-3 days between label incorporation in the mitotic pool (promyelocytes and myelocytes) and the arrival of labelled cells into the post-mitotic pool (metamyelocytes and banded neutrophils). A similar delay of 2-3 days was found between the occurrence of label in the banded neutrophil population and arrival of labelled cells in the mature neutrophil population, indicating that the cells spend at least 2-3 days in the banded neutrophil stage. Because of the observed delays and similarity in peak enrichments, a linear conveyor belt model (first in is first out principle) fits the data better than an exponential decay model.

This model, together with the differences in population sizes, implies that the circulatory neutrophil population has an average lifespan of at least 2 days. 


\section{Introduction}

The development, differentiation and maturation of neutrophils in the bone marrow has been extensively studied and is well described ${ }^{1}$. This is in contrast to the neutrophil progenitor proliferation rate and neutrophil lifespan. Most studies on neutrophil circulatory lifespan in vivo were performed in the 1950 s to 1970 s, reporting a circulatory half-life between 4 and $18 \mathrm{~h}$ (reviewed $\mathrm{in}^{2}$ ). These estimates were based on the rate at which labelled neutrophils disappear from the circulation. The interpretation of these studies was not without problems. The labels used were radio-active and/or toxic, such as the neurotoxin $\mathrm{DF}^{32} \mathrm{P}$ (diisopropyl fluorophosphates) or the radioactive nucleotide ${ }^{3} \mathrm{H}$-thymidin. The neutrophils were often labelled ex vivo and reinfused into the donor. Besides, because of the toxicity of the labels, the selected donors were often patients suffering from terminal diseases ${ }^{2}$. These shortcomings had probably affected neutrophil viability and/or distribution, and the resulting lifespan estimates are unlikely to represent normal neutrophil turnover in healthy homeostasis. This is supported by in vitro experiments demonstrating that neutrophils are capable of living much longer than the published in vivo half-lives ${ }^{3}$. In suspension, the mean half-life of a healthy neutrophil population was found to be around $48 \mathrm{~h}$, and in three-dimensional fibrin matrices this even increased to $59 \mathrm{~h}^{3}$. Furthermore, in zebrafish and mice it has been observed that photoconverted neutrophils can be traced at different tissue sites until at least $48 \mathrm{~h}$ after photoconversion $\mathrm{n}^{4,5}$. It is important to understand the correct circulatory lifespan in vivo to understand disturbed neutrophil kinetics in disease and to correctly predict the effect of therapies.

Fortunately, new labelling methods have been developed that allow safe, non-toxic labelling of human cells in situ using deuterium $\left({ }^{2} \mathrm{H}\right)$, a stable isotope of hydrogen $(\mathrm{H})$. This isotope is naturally occurring in very low levels, but can be artificially enriched in the human body by administering deuterated water $\left({ }^{2} \mathrm{H}_{2} \mathrm{O}\right)$ or deuterated glucose $\left(6,6-{ }^{2} \mathrm{H}\right.$-glucose) orally or intravenously. The rapid turnover of the body glucose pool allows for a pulse-chase design when using short term (6 hrs) deuterated glucose administration 6 . During the "pulse", the glucose will serve as a precursor for several newly synthesized molecules in the body. Most importantly, the glucose serves as a direct precursor of deoxyribose, and will thus be incorporated in the DNA of dividing cells. The label will stably remain detectable in the DNA of the cells and their progeny for the rest of the cell's life. After the pulse has ended, the enrichment of the cell population of interest is followed over time (the "chase"). At different time points the DNA is isolated from the cell population to measure deuterium enrichment with GCMS. From the enrichment of label over time the kinetics of the cell population can be estimated, aided by mathematical modeling?.

However, the use of mathematical modeling for neutrophil kinetics has proofed to be a challenge. This is the result of the absence of consensus of the basic mechanism underlying these kinetics. Here two opposing models in the literature have been presented: conveyor belt models and ordinary differential equation (ODE) models. Conveyor belt models as first suggested by Cartwright et $\mathrm{al}^{8}$ rely on 'the first in is the first out principle', whereas ODE models assume that maturation time of cells is a matter of chance ${ }^{9}$. Recently, an ODE model was developed by the group of Lahoz-Beneythez to explain the blood neutrophil labelling data on the basis of certain assumptions and 
without arguments to refute a first in is first out principle ${ }^{10}$. Using this ODE model, the authors came to the conclusion that the blood neutrophil half-life is around 19 hours. In this manuscript we will explain why this model is not biologically feasible and hence propose a new linear conveyor belt model instead. Our model is supported by our new deuterium labelling data from human neutrophil progenitors in the bone marrow. In addition, we also measured deuterium enrichment in neutrophils isolated from the oral cavity. Oral neutrophils have been described as a good model for extravasated neutrophils populating the tissues ${ }^{11,12}$.

\section{Materials and Methods}

\section{Deuterium labelling}

Bone marrow, blood, and oral rinse samples were obtained from 12 healthy male volunteers between the age of 18-30 years participating in a human endotoxemia study (ABR NL61136.091.17). The studies were approved by the ethics review board of the Radboud University Medical Center in Nijmegen, the Netherlands, and written informed consent was obtained from all study participants. Subjects were healthy as determined by medical history, physical examination, electrocardiography and hematological laboratory values. Subjects taking prescription drugs were excluded from the study.

All subjects received $6,6-{ }^{2} \mathrm{H}$-glucose (Cambridge Isotope Laboratories Inc., Tewksbury, MA, USA) at 1 to 6 days before the bone marrow aspiration, following a protocol described before ${ }^{18}$ with minor modifications. The subjects received $70 \mathrm{mg} 6,6-{ }^{2} \mathrm{H}$-glucose in twelve oral doses, administered every $30 \mathrm{~min}$, accumulating to a total pulse time of 6 hours.

The bone marrow sampling was performed by aspiration from the posterior iliac crest under local anesthesia. A total volume of $40 \mathrm{ml}$ was collected per aspiration into syringes prefilled with sodium heparin. The bone marrow aspirate (BM) was obtained around 8.00AM. Simultaneously with the bone marrow aspiration, blood samples (BL) were drawn from an arterial catheter (radial artery), using sodium heparin as an anticoagulant. For sampling of oral neutrophils the subjects vigorously rinsed their mouth for $30 \mathrm{sec}$ with $25 \mathrm{ml}$ of an isotonic saline solution, according to a protocol described before ${ }^{12}$.

\section{Cell isolation}

The full blood and heparin-diluted bone marrow suspension samples were counted on a Sysmex XN-450 automated hematology analyzer (Sysmex Corporation, Kobe, Japan). Then the erythrocytes in the bone marrow and blood samples were lysed using ice-cold lysis buffer ( $150 \mathrm{mM} \mathrm{NH}_{4} \mathrm{Cl}, 10 \mathrm{mM} \mathrm{KHCO}_{3}$ and $0.1 \mathrm{mM} \mathrm{Na}_{2}$ EDTA dissolved in $\mathrm{H}_{2} \mathrm{O}$; $\mathrm{pH}$ of 7.4). The remaining leukocytes were washed twice and resuspended in FACS staining buffer $(4 \mathrm{mg} / \mathrm{ml}$ human albumin [Sanquin, Amsterdam, The Netherlands] and $0.32 \%$ $(w / v)$ sodium citrate in PBS).

The oral rinse solutions were centrifuged for $10 \mathrm{~min}$ at room temperature and the cell pellet was filtered twice to remove debris and part of the contaminating epithelial cells. The resulting cell suspensions were counted using the Sysmex XN-450. 


\section{Flow cytometry}

For flow cytometric sorting, the cells were first incubated with anti-CD66b, anti-CD11b, anti-HLA-DR, anti-CD62L, anti-CD16, anti-CD193, anti-CD15, anti-CD56, and anti-CD45 fluorescent antibodies in FACS staining buffer on ice. Oral rinse cells were incubated only with anti-CD16 antibody. Bone marrow and blood cells were then stained with LIVE/ DEAD cell stain (Molecular Probes, Eugene, OR, USA) in PBS on ice. Cell populations were sorted using a FACSAria (BD, Franklin Lakes, NJ, USA). A minimum of 100,000 cells was sorted per population for DNA isolation. When enough material was available, cells were also sorted for preparation of cytospins.

Samples were analyzed on an LSRFortessa flow cytometer (BD). The results were analyzed using FlowJo software (FlowJo, LLC, Ashland, OR, USA).

For analysis of proliferation, immediately after aspiration and filtration a sample of bone marrow cells was treated with FACS ${ }^{\mathrm{TM}}$ Lysing Solution (BD) to lyse the erythrocytes and fix the leukocytes. Cells were washed, incubated with anti-CD66b, anti-CD11b, anti-CD16 and anti-Ki67 antibodies, and washed again. Shortly before analysis on an LSRFortessa flow cytometer (BD) propidium iodide was added to the cells to quantify DNA content.

\section{Measurement of deuterium enrichment by GC-MS}

From the sorted cell populations the DNA was isolated with the NucleoSpin Blood kit (Macherey-Nagel, Oensingen, Switzerland) according to the manufacturer's instructions. The DNA samples were stored at $-20^{\circ} \mathrm{C}$ until GC-MS measurement. The deuterium enrichment in the DNA was measured according to a protocol described before ${ }^{18,21}$. In short, the DNA was enzymatically hydrolyzed to deoxyribonucleotides, derivatized to PFTA derivatives, and analyzed with a combination of gas chromatography and mass spectrometry (GC-MS) with selective ion monitoring (SIM). The measurements were performed at $\mathrm{m} / \mathrm{z}$ ratios $435(\mathrm{M}+0$, unlabelled) and $437(\mathrm{M}+2$, labelled). Because for purine nucleotides the contribution of the salvage pathway is much lower than for pyrimidine nucleotides only the deoxyadenosine derivatives were measured ${ }^{22}$. Deoxyadenosine is mostly synthesized de novo and is thus more likely to derive from ${ }^{2} \mathrm{H}$-glucose.

The obtained $\mathrm{m} / \mathrm{z}$ ratios were calibrated against a standard range of known enrichment to calculate the percentage enrichment. The results in this chapter were corrected for background levels of deuterium ( $0 \%$ enrichment) but were not yet corrected for recycling of label through the nucleotide salvage pathway or for interdonor variability in label availability.

\section{Results and Discussion}

\section{Identification of the progenitor stadia of the neutrophil by flow cytometric analysis}

From twelve healthy volunteers bone marrow samples were obtained. During granulopoiesis in the bone marrow, the maturing neutrophil obtains its characteristic granulation and nuclear segmentation, and the expression levels of multiple surface markers gradually change. Flow cytometric analysis of the surface markers distinguished the maturation stadia of promyelocyte, myelocyte, metamyelocyte, 
banded neutrophil, and mature neutrophil in the bone marrow (Fig. 1). These different neutrophil populations in the bone marrow were enumerated as a percentage of the total neutrophil lineage (Figure 1B). Surprisingly the number of mature neutrophils (CD62 $\mathrm{L}^{\text {high }}$ and $\mathrm{CD} 2 \mathrm{~L}^{\mathrm{dim}}$ ) was 4.5 times larger than the banded neutrophil population $(p=0.002)$.

$\mathbf{A}$

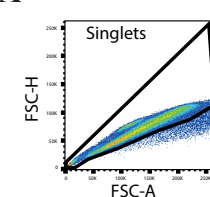

FSC-A
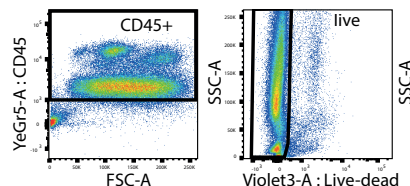

Violet3-A : Live-dead
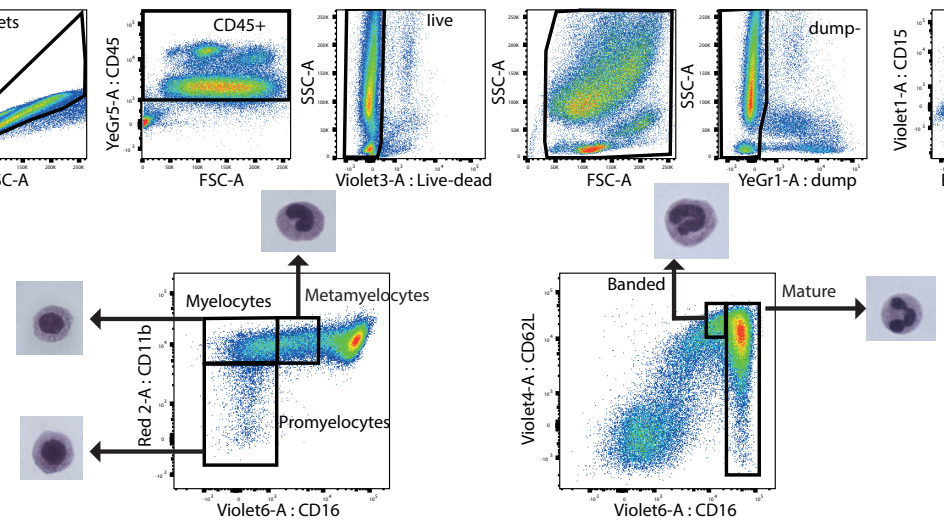

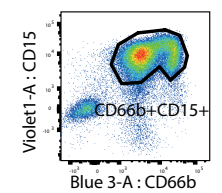

B
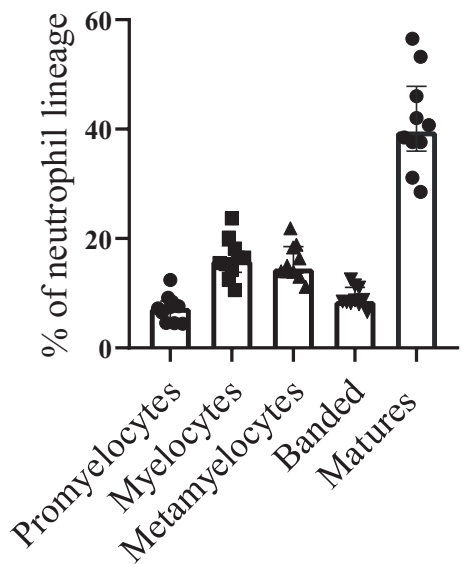

Figure 1: The stadia of granulopoiesis in the bone marrow were identified in flow cytometric analysis.

A: Flow cytometry strategy for distinguishing the different stadia of granulopoiesis from human bone marrow. The representative plots of one BM donor demonstrate the sequential gating steps. The representative micrographs of stained cytospin slides illustrate the morphology of the sorted populations. B: the relative abundance of neutrophils and their progenitors in the bone marrow is shown as a percentage of the total neutrophil lineage $\left.\left(\mathrm{CD} 6 \mathrm{~b}^{+} \mathrm{CD} 15^{+} \mathrm{CD} 193\right)^{-}\right)(\mathrm{n}=12)$. Median with interquartile range (IQR) is also indicated. 


\section{The kinetics of deuterium enrichment in homeostasis}

The healthy volunteers received deuterated glucose at different time points relative to the bone marrow aspiration to obtain the kinetics of the deuterium label over time (Fig. 2A). For every time point, ranging from 1 to 9 days after the labelling procedure, two volunteers were sampled. The samples of day 1 and day 8 after labelling were obtained from the same two donors by repeated bone marrow sampling, and the same accounted for the samples of day 2 and day 9 after labelling.

Fast uplabeling: The different stadia of the neutrophilic lineage were sorted with the gating strategy shown in Figure $1 \mathrm{~A}$ and the DNA was isolated. Within each cell population the deuterium enrichment was determined as the fraction of deuteriumlabelled DNA of the total amount of DNA (Figure 2C). As expected, shortly after the labelling procedure the label was only detected in the DNA of the mitotic pool (promyelocytes and myelocytes). In vivo data in man indicate a mean generation time of these mitotic neutrophil progenitors of around 14 hrs (Stryckmans, Nature 1966) showing the fast turnover of these cells ${ }^{13}$. This is also illustrated by the fact that a short pulse of deuterium-glucose $(6 \mathrm{hrs})$ lead to the maximal enrichment of 0.06 in the DNA of promyelocytes within one day. On day 3 after labelling, the metamyelocyte population became enriched illustrating a fast maturation in early granulopoiesis. Clearly, the fastest labelled promyelocytes and myelocytes had differentiated into metamyelocytes and had advanced into the post-mitotic pool. On day 4 after labelling, the fastest metamyelocytes had matured into banded cells. On day 6 , the mature population in the bone marrow contained a small percentage of labelled cells, that was very similar to the mature populations in the circulation and oral cavity. On day 8 the enrichment of all mature populations of neutrophils had increased to the same levels as found in promyelocytes. So, Figure $2 \mathrm{C}$ clearly shows that the label was following maturation through the post-mitotic differentiation steps in the neutrophilic lineage.

There is an important and surprising finding early in the differentiation of neutrophils that needs to be explained. The amount of ${ }^{2} \mathrm{H}$-enrichment in the DNA did not decrease in the promyelocytes from day 1 to day 2 despite the conclusion that the proliferation of the promyelocyte pool is fast (see above). A possible explanation for this is the putative misconception that promyelocytes and myelocytes in the bone marrow aspirate cannot be part of the non-dividing post-mitotic pool. There are in fact two findings that argue for non-dividing and only maturing (pro)myelocytes. Firstly, the percentage of cycling Ki67/PI positive cells is 'only' $10 \%$ in the total promyelocyte fraction and $<2 \%$ in the myelocytes (see figure $2 \mathrm{D}$ ). Secondly, there is no delay in the downlabeling of the two progenitor populations. It is, therefore, tempting to speculate that in the aspirate of the bone marrow the vast majority of the (pro)myelocytes have entered fast maturation rather than proliferation, and that the majority of the mitotic pool resides in the stroma of the bone marrow rather than the aspirate. If the aspiration and/or subsequent filtering step preferentially collected promyelocytes from the maturing pool, the measured enrichment was an underestimation of the in vivo enrichment of the total promyelocyte pool. This hypothesis is also supported by old studies implying the presence of a non-dividing 
so called lazy pool of (pro)myelocytes ${ }^{14}$. So all the data fit with an hypothesis that the majority of neutrophil progenitors from the mitotic pool that leave the stroma enter a differentiation/maturation program that is described by a conveyor belt. The finding that the peak enrichments are similar in all maturation steps supports this hypothesis. The actual promyelocyte enrichment in vivo is expected to peak during day 0 , when label is incorporated into fast dividing promyelocytes in the stroma of the bone marrow. However, this hypothesis needs to be addressed in future studies.

A

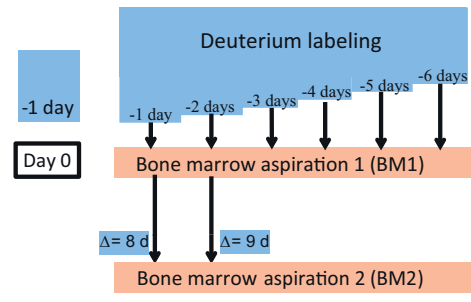

DNA enrichment

C

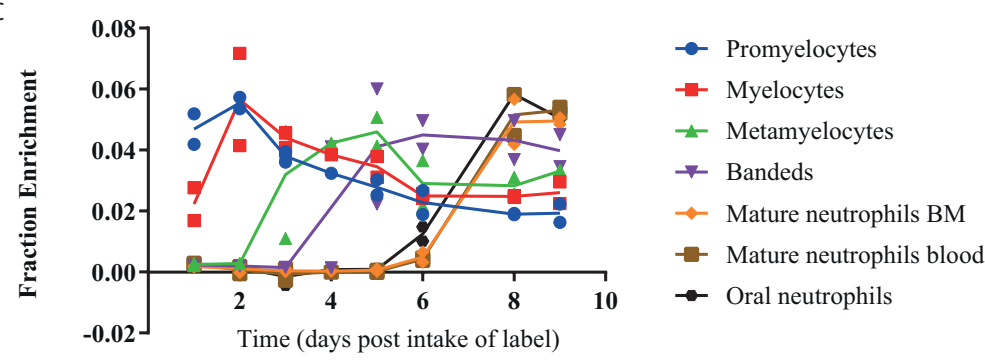

Plasma glucose

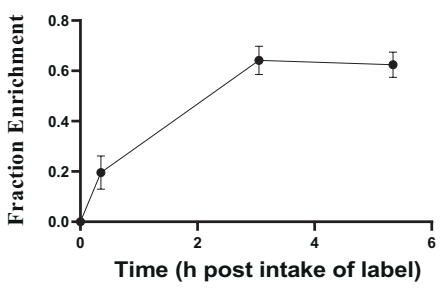

Time (h post intake of label)

$\mathrm{D}$

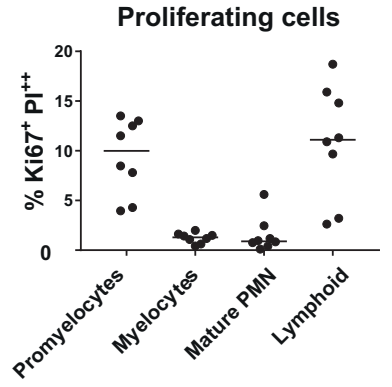

Figure 2: Kinetics of deuterium enrichment during homeostasis.

A: Schematic overview of the experimental design of the entire study. B: Deuterium enrichment of plasma glucose was measured before and at 3 time points during the intake of oral $6,6-{ }^{2} \mathrm{H}_{2}$-glucose to determine the availability of label for each individual C: Deuterium enrichment in the DNA of the sorted cell populations, expressed as the percentage of labelled DNA over total DNA. Twelve healthy subjects received a $6,6{ }^{-2} \mathrm{H}_{2}-$ glucose pulse over the course of 5,5 hours, and 1, 2, 3, 4, 5, 6, 8, or 9 days later bone marrow, blood and oral rinse were sampled. Neutrophilic populations were sorted from the BM1, BL1, and oral rinse samples of the 12 donors, as well as from the BM2, BL2, and oral rinse samples of 4 donors. Thus, a maximum of $\mathrm{n}=16$ measurements was obtained per celltype. The enrichment was measured by GC-MS and corrected 
for background levels of deuterium ( $0 \%$ enrichment), for plasma glucose and for the maximum amount of enrichment possible in granulocytes $(65 \% \text { of all dividing cells })^{6}$. Individual data points are shown. The line for each cell population connects the median values of each day with the other days. D: Percentage of actively proliferating cells in the bone marrow aspirates, measured in flow cytometry. Bone marrow cells were fixed immediately after aspiration. Promyelocytes, myelocytes and mature neutrophils were distinguished based on CD66b, CD11b and CD16 staining. Lymphoid cells were distinguished based on forward and side scatter characteristics. Proliferating cells were recognized as positive for Ki67 and having >2n DNA content based on propidium iodide fluorescence intensity.

Slow down-labeling: a fascinating but complex finding is the slow and comparable down-labeling of basically all neutrophil progenitors/mature cells. Apart from being another argument for the conveyor belt model (see below), it is quite a conundrum as in homeostasis production of cells equals death of the cells. A logical explanation is the presence of 2 or perhaps even more promyelocytes phenotypes characterized by a range of generation times that feed into the conveyor belt with the majority being short (fast cells) but range into long times (slow cells). The 'first in equals first out' principle leads to the situation that on a perfect conveyor belt the enrichment of these fast and slow cells will be similar for all progenitors on the conveyor as can indeed be seen in figure 2C. Following this logic, the area under the curve of the different progenitors is a measure of the generation time of a certain population as the pulse merely follows differentiation. This leads to the conclusion that at equal sizes of the populations the generation time of the banded cells would equal the generation time of mature neutrophils. On the other hand, when the mature neutrophil pool is larger than the banded pool (see Figure 1B) this will lead to the conclusion that generation time of neutrophils relates to that of the banded population by the formula: $\mathrm{Nm} / \mathrm{Nb} \times 2$ days in which $\mathrm{Nm}=$ total number mature neutrophils; $\mathrm{Nb}=$ total number of banded cells, and 2 days being the fastest generation time of banded cells. This latter generation time equals the time between max enrichment in mature cells minus the time max enrichment of the banded cells. These considerations emphasize the importance of an accurate determination of the total pools of mature and banded neutrophils. In our study this equals 1: 4.5 for banded vs mature cells (see figure 2D). Other studies ${ }^{8,15}$ came to other numbers, but no studies have indicated that banded cells were in access to mature cells. This leads to the conclusion that the generation time of a neutrophil is minimally 2 days (see also final section). The last issue to be explained is the finding that the peak height of the mature neutrophils did not "dilute" as a result of a larger number of segmented neutrophils present in the total neutrophil compartment. A possible reason could be that bone marrow aspirates overestimated the relative number of mature neutrophils because of hemodilution (segmented neutrophils from the blood were also aspirated in the syringe). Indeed, bone marrow aspirates consistently contain a larger proportion of mature neutrophils than bone marrow biopsies ${ }^{16}$.

\section{Compartmentalization of labelled neutrophils}

Counterintuitively, the kinetics of neutrophils isolated from the oral cavity equaled that of peripheral blood. This can be explained by multiple not mutually exclusive hypotheses: 1 the oral neutrophils had spent little time in the circulation between leaving the bone marrow and entering the oral cavity, since the label kinetics did not show a delay between the circulatory mature population and the oral neutrophil 
population. Instead, the kinetics of the mature neutrophils in the bone marrow, the circulation, and the oral cavity was highly similar. The similarity in uplabelling suggests that the post-mitotic pool ends with the differentiation from banded to mature neutrophil. Directly after this final step the mature neutrophil enters a compartment with complete exchange as the kinetics are very similar. Thus, the banded neutrophils of the bone marrow feed in to all these populations.

From the dissimilar population size but similar enrichment peak of the three mature neutrophil populations (bone marrow, circulatory and oral) follows that the average lifespan of the three populations is similar. If all three compartments have the same death rate, the same percentage of dead neutrophils needs to be replaced by newly produced neutrophils every day. Hence, while the label passes from the banded to the mature stadium, the dilution or concentration factor will be the same in all three compartments. Consequently, the enrichment peaks of the three neutrophil populations will be the same.

\section{Mathematical modeling. Is ODE the right model?}

The downside of in vivo deuterium labelling is that incorporation of label happens in progenitors in the bone marrow. So, when label is measured in blood, it is impossible to distinguish between labelled cells surviving in the blood from labelled cells that have just appeared from the bone marrow ${ }^{10,17}$. Mathematical modeling is often used to comprehend the data, but unfortunately does not provide full clarity in neutrophil kinetics ${ }^{10}$. Different mathematical models give different conclusions. For example: in lymphocyte kinetics an Ordinary Differential Equation model (ODE) is used to calculate average turnover rate $(p)$ and average rate at which label is lost from the population $(d)^{9}$. In this model both the rate of turnover (or proliferation) and rate of label disappearance is equal and assumed to be stochastic. Although this model is biologically feasible in lymphocyte kinetics, for granulocyte kinetics this might not be the case. That is, the ODE model gives fits of the data with two equally possible estimations as was explained in the article of Lahoz-Beneytez et $\mathrm{al}^{10}$. One estimations assumes that the bone marrow is the slowest compartment, which means that the time between cell divisions is slow (mean of about 99 hours) and/ or very variable ${ }^{10}$. The second estimation assumes that the blood is the slowest compartment, which means that time between different cell divisions in the bone marrow is short ( $<1$ hour). However, both estimations are physiologically untenable (see below).

The first situation, a slow bone marrow compartment, is challenged by the fact that it has been already shown that time between different cell divisions in the bone marrow is not as long as the model suggests: the time between two different myelocyte divisions is around 14 hours in both man and rodents ${ }^{13,18}$. Another argument against a stochastic/ ODE model is that it has been shown that daughter cells of progenitors cells divide with a constant speed without signs of large variations ${ }^{13,19}$. Besides, the first estimation of Lahoz-Beneytez et al. did not account for the neutrophil compartment in the bone marrow (Figure 1B) and the fact that not all progenitors divide (lazy pool) ${ }^{20}$. Finally, their stochastic (ODE) model assumes that all neutrophil progenitors (independent whether they are GMPs, promylocytes or myelocytes) have the same post mitotic pool transit time (time from progenitor to fully mature neutrophil). This concept has been challenged before by other authors ${ }^{21}$. 
The second situation, a fast bone marrow compartment, only fits the data if the (circulatory) segmented neutrophil pool is five times bigger than the mitotic precursors (promyelocytes and myelocytes), which is not wat is reported in the literature or found in our study (Figure 1B) ${ }^{10}$.

Both situations described above give a quite different neutrophil half-life: 19 hours versus $>72$ hours dependent on which compartment (bone marrow versus blood) is the slowest compartment ${ }^{10}$.

In conclusion, a stochastic (ODE) model to describe neutrophil kinetics is incorrect, because the time of division between different neutrophil progenitors is not variable or longer than 24 hours; the neutrophil progenitor pool is not larger than the mature neutrophil pool and the post mitotic pool transit time is not similar for all progenitors.

\section{The linear conveyor belt model as an alternative model for neutrophil kinetics}

An alternative model is the linear conveyor belt model, as was first described by Cartwright and his colleagues in $1964^{8}$. This model assumes that cells divide and differentiate in a subsequent order, which also means that the cell that entered the circulation first, will leave the circulation first. So, according to this "first in, first out" principle, the oldest metamyelocytes in the population will be the first metamyelocytes to differentiate into banded neutrophils and so forth. The idea that the maturity of neutrophil progenitors depends on the cellular age fits well with the gradual acquisition of characteristics such as CD16 expression (Fig. 1B). and nuclear segmentation.

Another characteristic of this model is that the labelled population does not have the same chance to proceed to the next stadium as does the unlabelled population. E.g. on day 2 after label intake, any labelled cells in the myelocytes population will still be young myelocytes. The enrichment measured in the myelocyte population as a whole is high, but because only the oldest myelocytes, which were already produced before the pulse, are ready to advance to the metamyelocyte stadium, zero label is transmitted to the metamyelocyte stage (Figure $2 \mathrm{C}$ ). On day 3 after labelling, the labelled myelocytes have become one day older and some are now ready to advance to the next stage. Consequently, the amount of label in the myelocytes population is decreasing, and the amount of label in the metamyelocyte population is increasing. In conclusion, even though the departure rate of the cells is equal at any given time point, the disappearance rate of the label is not. In contrast, if a random selection of myelocytes would advance to the metamyelocytes stadium every day, label would appear in the metamyelocytes population already on day 1 and 2 after labelling. Furthermore, if a random selection of banded neutrophils would advance to the mature stadium every day, label would most likely appear in the mature population on day 4 and 5 after label intake. Since, we have shown that ${ }^{2} \mathrm{H}$-label is equally transmitted from one progenitor to the other in time (Figure $2 \mathrm{C}$ ), the conveyor belt model seems to be a more biologically realistic model than the ODE model.

In conclusion, in contrast to the above discussed ODE model, the time between two different divisions is not stochastic but fixed ${ }^{19}$. Because of the linear behavior of cell development and kinetics, terms such as half-life are, therefore, incorrect. Better terminology is "mean time in circulation" or "neutrophil lifespan". 


\section{Using the linear conveyor belt model, we propose a neutrophil lifespan of at least} 2 days

A computational method to fit the data with a linear conveyor belt model has unfortunately not been developed yet. Using the data from this study in combination with several biologically feasible assumptions, we can however deduct a blood neutrophil lifespan of at least 2 days.

The first assumption is that the entire segmented neutrophil pool (both blood and bone marrow cells) are originating from the banded neutrophil pool in the bone marrow. The second assumption is that both blood and bone marrow neutrophils have the same lifespan (Figure 2C). The third assumption is that the number of all neutrophil progenitors and mature neutrophils in both pools is stable during homeostasis.

Now, we have shown that there is a ${ }^{2} \mathrm{H}$-label delay of at least 2 days between the mature and the banded neutrophils $\left({ }^{2} \mathrm{H}\right.$-label appears in the banded neutrophils at day 4 and at day 6 for the mature neutrophils). This was similarly shown in our earlier study in which we showed that after endotoxemia label appeared in the banded neutrophils 2 days earlier than it did in the mature neutrophils ${ }^{22}$. With other words, banded neutrophils need at least 2 days to become mature neutrophils. So, in order to prevent an increase in the absolute number of mature neutrophils, the lifespan of all mature neutrophils should also be at least 2 days assuming that the pool sizes of banded cells and mature neutrophils are similar.

This linear conveyor belt model is most likely also applicable for eosinophil progenitors in the human bone marrow ${ }^{23}$.

We aim to apply modeling to our data in the near future, to derive more exact estimates of the lifespan of the mature neutrophil. It is therefore that this thesis focuses more on the description of kinetics rather than the calculations of lifespans.

\section{Acknowledgements}

The authors thank Jeroen van Velzen and Pien van der Burght for help with the flow cytometry sorting. 


\section{References}

1. Lawrence, S. M., Corriden, R. \& Nizet, V. The Ontogeny of a Neutrophil: Mechanisms of Granulopoiesis and Homeostasis. Microbiol. Mol. Biol. Rev. 82, (2018).

2. Tak, T., Tesselaar, K., Pillay, J., Borghans, J. A. M. \& Koenderman, L. What's your age again? Determination of human neutrophil half-lives revisited. J. Leukoc. Biol. 94, 595-601 (2013).

3. van Grinsven, E. et al. A comprehensive three-dimensional assay to assess neutrophil defense against bacteria. J. Immunol. Methods 462, 83-90 (2018).

4. Dixon, G., Elks, P. M., Loynes, C. A., Whyte, M. K. B. \& Renshaw, S. A. A Method for the In Vivo Measurement of Zebrafish Tissue Neutrophil Lifespan. ISRN Hematol. 2012, 1-6 (2012).

5. Hampton, H. R., Bailey, J., Tomura, M., Brink, R. \& Chtanova, T. Microbe-dependent lymphatic migration of neutrophils modulates lymphocyte proliferation in lymph nodes. Nat. Commun. 6, 7139 (2015).

6. Macallan, D. C. et al. Measurement of proliferation and disappearance of rapid turnover cell populations in human studies using deuterium-labeled glucose. Nat. Protoc. 4, 1313-27 (2009).

7. Ahmed, R. et al. Reconciling Estimates of Cell Proliferation from Stable Isotope Labeling Experiments. PLOS Comput. Biol. 11, e1004355 (2015).

8. Cartwright, G. E., Athens, J. W. \& Wintrobe, M. M. The kinetics of granulopoiesis in normal man. Blood 24, 780-803 (1964).

9. Vrisekoop, N. et al. Sparse production but preferential incorporation of recently produced naive T cells in the human peripheral pool. Proc. Natl. Acad. Sci. U. S. A. 105, 6115-20 (2008).

10. Lahoz-Beneytez, J. et al. Human neutrophil kinetics: modeling of stable isotope labeling data supports short blood neutrophil half-lives. Blood 127, 3431-3438 (2016).

11. Cheretakis, C., Dror, Y. \& Glogauer, M. A noninvasive oral rinse assay to monitor engraftment, neutrophil tissue delivery and susceptibility to infection following HSCT in pediatric patients. Bone Marrow Transplant. 36, 227-32 (2005).

12. Akpek, G., Knight, R. D. \& Wright, D. G. Use of oral mucosal neutrophil counts to detect the onset and resolution of profound neutropenia following high-dose myelosuppressive chemotherapy. Am. J. Hematol. 72, 13-9 (2003).

13. Stryckmans, P., Cronkite, E. P., Fache, J., Fliedner, T. M. \& Ramos, J. Deoxyribonucleic acid synthesis time of erythropoietic and granulopoietic cells in human beings. Nature 211, 717-20 (1966).

14. Mary, J. Y. Normal human granulopoiesis revisited. II. Bone marrow data. Biomed. Pharmacother. 39, 66-77 (1985).

15. Donohue, D. M., Reiff, R. H., Hanson, M. L., Betson, Y. \& Finch, C. A. Quantitative Measurement of the Erythrocytic and Granulocytic Cells of the Marrow and Blood1. J. Clin. Invest. 37, 1571-1576 (1958).

16. Loken, M. R., Chu, S.-C., Fritschle, W., Kalnoski, M. \& Wells, D. A. Normalization of bone marrow aspirates for hemodilution in flow cytometric analyses. Cytometry B. Clin. Cytom. 76, 27-36 (2009).

17. Li, K. W., Turner, S. M., Emson, C. L., Hellerstein, M. K. \& Dale, D. C. Deuterium and neutrophil kinetics. Blood 117, 6052-3; author reply 6053-4 (2011).

18. Constable, T. B. \& Blackett, N. M. The cell population kinetics of neutrophilic cells. Cell Tissue Kinet. 5, 289-302 (1972). 
19. Tamar Tak, Giulio Prevedello, Gaël Simon, Noémie Paillon, Ken R. Duffy, L. P. Simultaneous tracking of division and differentiation from individual hematopoietic stem and progenitor cells reveals within-family homogeneity despite population heterogeneity. bioRxiv (2019).

20. Dresch, C., Troccoli, G. \& Mary, J. Y. Growth fraction of myelocytes in normal human granulopoiesis. Cell Tissue Kinet. 19, 11-22 (1986).

21. Dancey, J. T., Deubelbeiss, K. A., Harker, L. A. \& Finch, C. A. Neutrophil kinetics in man. J. Clin. Invest. 58, 705-15 (1976).

22. Tak, T. et al. Human CD62Ldim neutrophils identified as a separate subset by proteome profiling and in vivo pulse-chase labeling. Blood 129, 3476-3485 (2017).

23. Hassani, M. et al. Characterization of the phenotype of human eosinophils and their progenitors in the bonemarrow of healthyindividuals. Haematologica haematol.2019.219048 (2019) doi:10.3324/haematol.2019.219048. 



\section{Chapter 3}

\section{On the origin of low-density neutrophils}

Hassani M. ${ }^{1, *}$, Hellebrekers $P .{ }^{1,2 *}$, Chen $N^{1}{ }^{1}$ van Aalst $C .{ }^{1}$, Bongers $S .{ }^{1,2}$, Hietbrink $F^{2}$, Koenderman L. ${ }^{1,5}$ and Vrisekoop N. ${ }^{1,5}$

1. Department of Respiratory Medicine and Center for Translational Immunology, University Medical Center Utrecht

2. Department of Surgery, University Medical Center Utrecht

* These authors contributed equally to the manuscript.

\$ These authors contributed equally to the manuscript. 


\section{Abstract}

Nowadays the term low(er) density neutrophils (LDN) is used to describe a specific subset of neutrophils with a certain buoyant density, but these LDNs are characterized by a wide variety in both functional and morphological properties, especially in disease. Both immature and activated mature neutrophils are found in the LDN fraction.

Here we elaborate on the origin of LDNs to better understand the variation found in literature. Supplemented with original data, we test the hypothesis that buoyant density of neutrophils is characterized by a spectrum which as a whole shifts to a lower density after activation. Both the $20 \%$ highest density (HDNs) and $20 \%$ lowest density (LDNs) neutrophils from healthy donors were isolated by Percoll of different densities. Using this method we found that LDNs were significantly better in T cell suppression and bacterial containment than their $20 \%$ highest density counterparts. We found no statistically relevant differences in neutrophil survival or bacterial phagocytosis. Stimulation of healthy donor neutrophils with fMLF induced LDNs co-segregating with PBMC after Ficoll separation. These in vitro induced LDNs showed increased activation markers compared to HDNs and were comparable to the activation markers found on the LDN fraction seen in patients with chronic inflammatory conditions such as present in cancer patients.

This all fits with the hypothesis that the density of neutrophils is distributed in a spectrum partially coupled to maturation. Additionally a shift in this spectrum can be induced by in vitro stimulation or by activation in disease. 


\section{Introduction}

Neutrophils are main actors in the innate immune system. Until recently neutrophils were thought to belong to a relative homogeneous population of cells. However, an increasing number of studies now show heterogeneity in morphology, phenotype, function or a combination of these factors ${ }^{1}$. One of the subtypes of neutrophils identified and studied is the low-density neutrophil (LDN). It was first recognized in 1986, when neutrophils co-segregated with mononuclear cells after density-gradient isolation in systemic lupus erythematosus and rheumatoid arthritis patients ${ }^{2}$. Thereafter, the presence of LDNs have been shown in several, mostly chronic, diseases ${ }^{3}$.

Although the term ' $L D N$ ' is used nowadays to indicate a distinct neutrophil subset, it actually refers to a wide variety of neutrophils in different pathological circumstances. In fact, LDNs are poorly defined when it comes to morphology and function. For example, both pro-inflammatory and anti-inflammatory properties have been linked to LDNs in inflammatory diseases ${ }^{3}$. Also, both immature (banded nucleus), as well as hypersegmented neutrophils have been identified in the mononuclear layer ${ }^{3}$. Interpretation of the above mentioned studies in terms of ontogeny of the LDNs in vivo is hampered by the fact that the onset of inflammatory disease is difficult to determine. It is important to study the kinetics of LDNs to discriminate between activation and a separate lineage. In this hybrid review we have combined existing data, found in the literature, with new original experimental data in order to better understand the origin and functional properties of LDNs in health and disease.

\section{Density is a spectrum in neutrophils}

In 1983, Pember et al. showed that heterogeneity of density with a bell-shaped distribution exists within the healthy human circulating neutrophil pool ${ }^{4}$. In addition, in this study density and mean cell volume were found to be connected. A similar distribution of different densities was also found in elicited and non-elicited murine neutrophils, and in neutrophils in allergic human subjects ${ }^{5,6}$.

More recently, Herteman et al. studied LDNs in asthmatic and healthy horses and found that, although their numbers varied between health and disease, the functional characteristics were similar regardless of health status ${ }^{7}$. So, LDNs in asthmatic and healthy horses both had an increased capacity to produce neutrophil extracellular traps, and were similarly more sensitive to activation by phorbol-12-myristate-13-acetate ${ }^{7}$. LDNs in both conditions had more $\mathrm{N}$-formylmethionine-leucyl-phenylalanine (fMLF) receptors and were smaller in size, which according to the authors made them intrinsically different. LDNs were described in several studies in healthy volunteers as well ${ }^{8,9,10}$.

Besides a distribution of buoyant densities, other studies have shown functional heterogeneity within the circulating neutrophil pool of healthy humans ${ }^{11,12,13}$. For instance, in the studies of Eggleton et al. a similar Gaussian-shaped profile on the basis of different cell surface electrical charge was found ${ }^{12,13}$. The presence of heterogeneity in the density and functionality in healthy neutrophils prompted us to test the hypothesis that a spectrum of neutrophil densities associated with different functionalities are already present in the circulation of healthy humans. 
Unstimulated neutrophils from healthy volunteers were centrifuged over different Percoll densities between 1.079 and $1.083 \mathrm{~g} / \mathrm{ml}$. Similarly as in the study by Pember et al. $^{4}$ the number of cells found in the upper ring increased when higher Percoll densities were used for centrifugation, but not all cells shifted up (Figure 1A). Some donor variation was present but in all donors more cells stayed in the ring of Percoll with a high density $(1.083 \mathrm{~g} / \mathrm{ml})$ compared to the Percoll with a low density $(1.079 \mathrm{~g} /$ $\mathrm{ml}$ ) (Figure 1A). This proofed that a natural spectrum in densities was present in the circulating neutrophil pool, even in healthy individuals. Although neutrophil function is known to change with age ${ }^{14}$, we did not find a correlation between total numbers of LDNs or HDNs and the donors age (Supplementary Figure 1). Next, we tested whether the $10-20 \%$ of the lowest density neutrophils (LDNs, typically found on top of Percoll gradient with a density of $1.081 \mathrm{~g} / \mathrm{ml}$ ) had different functional properties compared to the $10-20 \%$ of the highest density neutrophils (HDNs, typically found on the bottom of Percoll gradient with a density of $1.083 \mathrm{~g} / \mathrm{ml}$ ). All neutrophils combined were used as a control, because they also contain the cells with the intermediate densities.

First, a containment assay was used to compare LDN and HDN functionality, as described by us before ${ }^{15}$. This assay mimics the in vivo situation and tests several neutrophil functions: chemotaxis, phagocytosis, bacterial killing and neutrophil survival. The delay in GFP-labeled bacterial outgrowth (lag time) serves as a measure for the antibacterial capacity of the different neutrophils. There was a clear difference in the containment capacity of S. aureus of neutrophils in favor of the $20 \%$ lowest density neutrophils (Figure 1B). The extent of the advantage of the LDNs compared to the HDNs varied between the experiments but was always in favor of the LDNs. The containment capacity of the total neutrophil fractions showed a lag time comparable to LDNs. This implies that HDNs are less capable of containing bacteria compared to the other neutrophils in the density spectrum. To rule out the overrepresentation of eosinophils in the $20 \%$ highest density fraction, the experiment was repeated after sorting out eosinophils by flow cytometry cell sorting, after which the same defect in bacterial containment capacity was seen (data not shown). Knowing that phagocytosis was comparable between the fractions (Supplementary Figure $2 \mathrm{~A}-\mathrm{C}$ ) the enhanced containment of bacteria by LDN was suggested to be an effect of either bacterial killing or neutrophil survival. To differentiate between these possibilities a survival assay was performed which did not show any difference between the different fractions (Supplementary Figure 2D). Therefore, it can be argued that the advantage seen in this bacterial containment assay is due to different capacity in bacterial killing. 
A

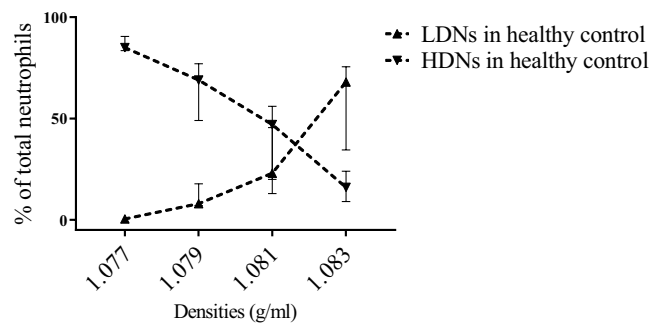

C

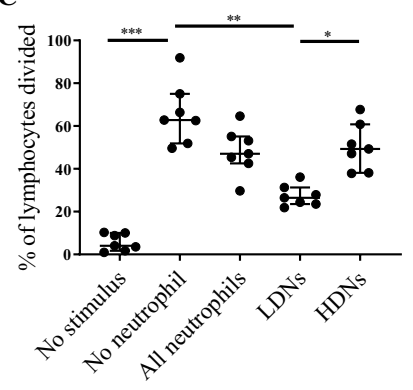

$\mathbf{E}$

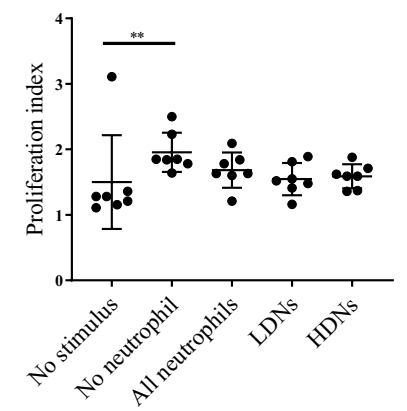

B

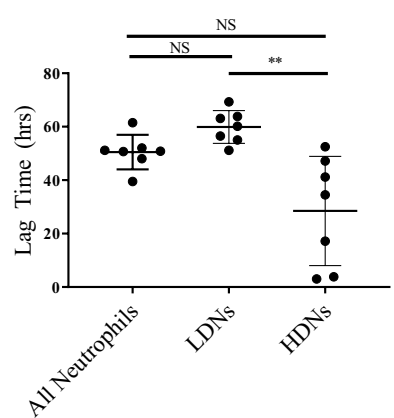

D

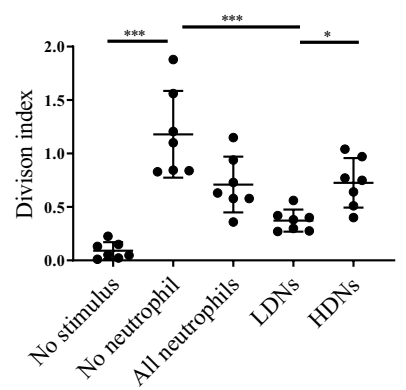

Figure 1. Unstimulated neutrophils display a spectrum of densities.

Neutrophils were first isolated using Ficoll density centrifugation. Thereafter isolated neutrophils were centrifuged on top of Percoll with different densities. A. Depicted is the percentage of total neutrophils in the 'PBMC layer' after density centrifugation with different densities of Percoll $(n=9)$. B. The lag time in which HDN and LDN contain growth of GFP labeled $S$. Aureus $(n=7)$. C. The percentage of the original population of lymphocytes that divided after stimulation in the absence of neutrophils, in the presence of all neutrophils and in the presence of either LDN or HDN of the same donor $(n=7)$. $D$. Division index (average number of cell divisions that a cell in the original population has undergone) of lymphocytes under the same conditions as C. E. Proliferation index (the total number of divisions divided by the number of cells that went into division) of lymphocytes under the same conditions as $\mathrm{C}$. For all graphs median \pm IQR\% is shown. Data are analyzed using Friedman test without correction for multiple comparisons. C-E all conditions were tested, but only statistically significant results are indicated. 
Next, we tested putative differences in the capability to suppress proliferation of lymphocytes. It has been previously shown that different neutrophil phenotypes have the capability to suppress lymphocyte proliferation ${ }^{16,17}$, which has also been linked to LDNs found in a variety of acute and chronic conditions ${ }^{18,19,20,21}$. Indeed, LDNs isolated from healthy donors, showed a better capability to suppress lymphocyte proliferation after 4 days, especially when they are compared with the HDNs (Figure 1C-E). Interestingly, the division index - which takes all cells into account- is clearly different (Figure 1D), while the proliferation index (Figure 1E) -which only takes the dividing cells into account- is not. It is therefore possible that when lymphocytes have "escaped" the suppression, they do not seem to be sensitive for neutrophil mediated suppression anymore. So, from above mentioned data it can be concluded that a property to suppress proliferation of lymphocytes is linked to the intrinsic buoyant density of neutrophils from healthy donors.

\section{Activation of neutrophils in vitro leads to a shift of density}

Both human and murine neutrophils change density when activated by endotoxinactivated serum or fMLF in vitro ${ }^{4}$. Interestingly, this change in density still resulted in a distribution of densities with a bell shape ${ }^{4}$. This finding suggests that activation of neutrophils leads to a change of density for all cells, but only neutrophils that are in the lower density of the Gaussian distribution prior to activation will segregate in the PBMC fraction after Ficoll centrifugation.

Other proof that lower density granulocytes are an effect of activation was shown when neutrophils were incubated with Mycobacterium tuberculosis in vitro ${ }^{22}$. Since LDNs were also found in patients infected with Mycobacterium tuberculosis, it is very plausible that a direct interaction between neutrophils and the bacterium leads to a decrease in density 22 .

Further complexity is introduced when taking into account that neutrophil density gradually decreases when the processing of neutrophils is delayed for more than 6 hours $^{23}$. This "spontaneous" density shift of neutrophils in vitro is likely to contribute to the wide variety of LDN numbers found between different experimental setups.

To reproduce that in vitro activation shifts the buoyant spectrum of freshly isolated neutrophils from healthy volunteers, we stimulated neutrophils with $\mathrm{fMLF}$ and platelet activating factor (PAF) in a concentration range between $10 \mu \mathrm{M}$ and $1 \mathrm{pM}$ (Figure 2A-B). Indeed, stimulation of neutrophils in vitro caused a decrease in density in at least part of the neutrophils. After fMLF stimulation, the fraction of the neutrophils that were found in the PBMC fraction because of a decreased buoyancy $<1.077 \mathrm{~g} / \mathrm{ml}$ followed a bell shaped curve, with the maximum shift at $0.01 \mu \mathrm{M}$ (Figure 2A). In contrast, when stimulated with PAF, in the density shift appeared in a concentration dependent manner with the highest concentration of PAF resulting in the highest percentage of LDNs (Figure 2B). These results complicate the understanding of LDNs even further, because apparently not only the stimulating agent itself is important in the number of LDNs that are generated, but also the concentration of the stimulus. 
A

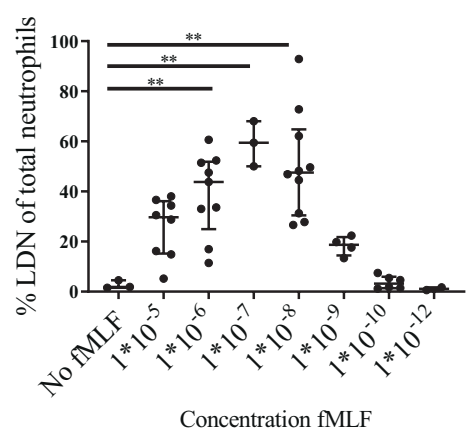

C

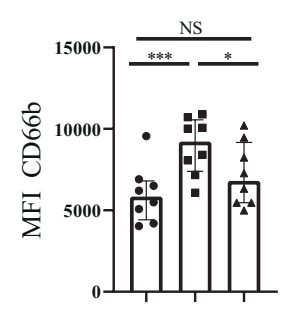

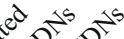

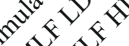

30

कर
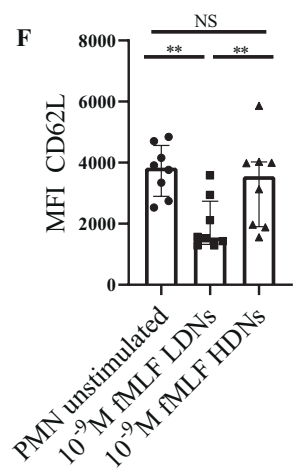

o 0
D
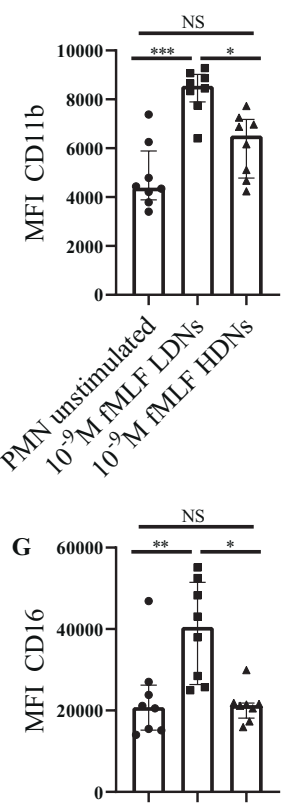

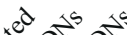

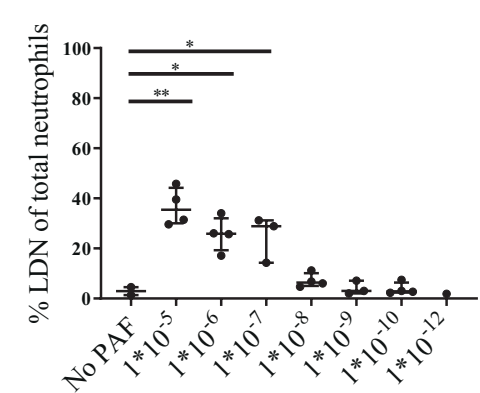

Concentration PAF

E
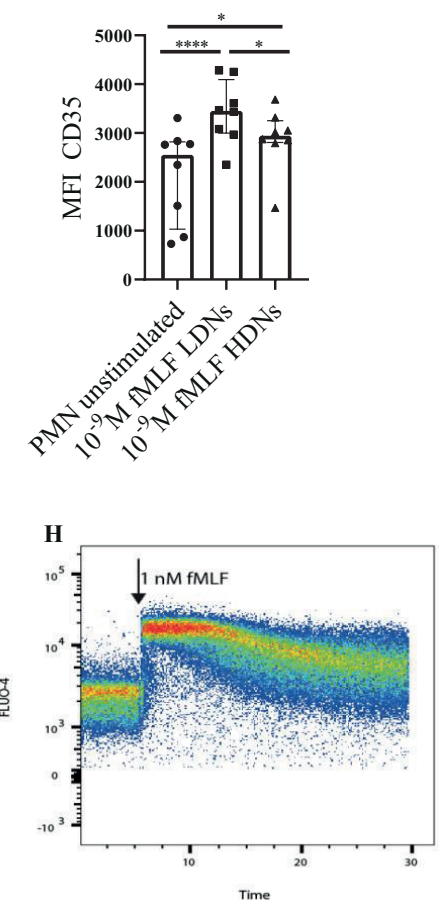

Figure 2. Density shift of neutrophils after stimulation.

Depicted is the percentage of neutrophils in the PBMC fraction after stimulation of neutrophils with $\mathbf{A}$. fMLF or B. PAF C-G Median fluorescence intensity of activation markers on unstimulated neutrophils and LDNs and HDNs after $1 \mathrm{nM}$ fMLF stimulation ( $\mathrm{n}=8$ ). C. CD66b. D. CD11b E. CD35 F. CD62L G. CD16. H. FLUO-4 signal for gated neutrophils is shown before and after adding $1 \mathrm{nM}$ fMLF. Data are depicted as median with interquartile range (IQR). Data were analyzed using Friedman test without correction for multiple comparison. A-B all conditions were tested, but only statistically significant results are indicated. 
Next, fMLF stimulated neutrophils were analyzed after Ficoll Paque density centrifugation, using a multicolor flow cytometry panel. This experiment showed that after $1 \mathrm{nM}$ fMLF stimulation, all neutrophils (LDNs and HDNs) show a change in expression of activation markers ( Figure 2C-G). However, there was a remarkable difference in activation markers between LDNs and HDNs. LDNs showed a higher expression of CD66b, CD11b, CD35 and CD16 and a lower CD62L expression after $1 \mathrm{nM}$ fMLF stimulation (Figure $2 \mathrm{C}-\mathrm{G}$ ). This is in contrast with unstimulated neutrophils of different densities, which did not show a difference in activation markers between LDNs and HDNs (Supplementary Figure 3). A higher concentration of fMLF $(0.1 \mu \mathrm{M})$ also led to an increase of activation markers in both density fractions, but the differences between LDNs and HDNs were less pronounced (Supplementary Figure 4). This could mean that during homeostasis, certain neutrophils are more responsive to a lower concentration (1 $\mathrm{nM}$ ) of fMLF with a subsequent shift in density of only these cells. However, since all neutrophils show a $\mathrm{Ca}^{2+}$ influx with this low concentration of fMLF (Figure $2 \mathrm{H}$ ), another possible explanation could be that all cells shift in density but, as described in paragraph 2 , only the cells on the lower end of the spectrum end up in the PBMC fraction.

\section{LDNs in disease are heterogeneous}

The appearance of neutrophils in the PBMC fraction after Ficoll-Paque centrifugation are described in several inflammatory diseases. However, when different patient groups and different studies are compared, these cells are quite variable in both phenotype and functionality 3,9,20,24,25. In the following paragraphs, the different subsets of LDNs are described, supplemented with own data. A more extensive description of LDN in inflammatory diseases have been summarized recently in an excellent review by Scapini et al $^{3}$.

\section{Are LDNs mainly immature neutrophils?}

In the human bone marrow, a progressive increase of buoyant density was described for maturation of cells within the granulocyte lineage ${ }^{26,27}$. Banded and segmented neutrophils displayed a density above $1.080 \mathrm{~g} / \mathrm{ml}$ while promyelocytes and myelocytes exhibit a density below $1.080 \mathrm{~g} / \mathrm{ml}^{27}$.

Both acute and chronic inflammatory conditions often lead to an enhanced (emergency) granulopoiesis in the bone marrow ${ }^{28,29}$ that will subsequently lead to the appearance of immature neutrophils in the circulation, often referred as a left shift ${ }^{30}$. Similarly, in a model of systemic inflammation, human experimental endotoxemia also leads to the appearance of banded neutrophils in the circulation ${ }^{31}$. Since neutrophil progenitors have a lower buoyant density, it is not surprising that in certain inflammatory conditions such as cancer or sepsis these immature neutrophils are found in the PBMC fraction ${ }^{19,32,33}$.

To test whether density is linked to maturation of neutrophils in healthy volunteers, we used a multicolor flow cytometry analysis on unstimulated neutrophils after Percoll density centrifugation. Although their numbers were small in healthy donors, a striking difference was found in the amount of CD16 ${ }^{\text {dim }} /$ CD62 $\mathrm{L}^{\text {high }}$ banded neutrophils in the $20 \%$ lowest density neutrophils (median of $2.7 \%$ in LDNs versus median of $0.24 \%$ in HDNs, Figure $3 \mathrm{~A})^{34}$. 

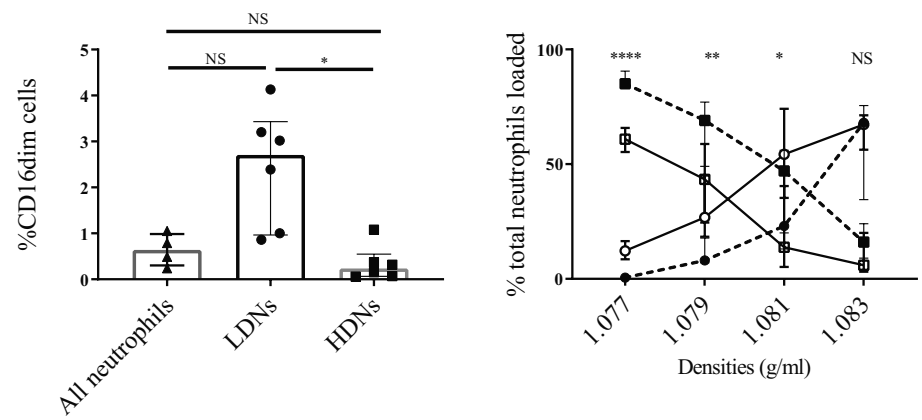

-• LDNs in healthy control

-a. HDNs in healthy control

- - LDNs after LPS

— HDNs after LPS

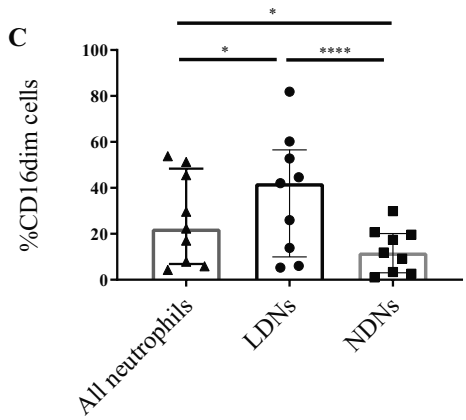

Figure 3. $\mathrm{CD} 16^{\mathrm{dim}}$ neutrophils (banded nucleus) are more abundant in the LDN fraction.

A. Depicted is the percentage of $\mathrm{CD} 16^{\mathrm{dim}}$ neutrophils of healthy donors in all neutrophils, LDN and HDN $(n=6)$. B. Presented is the percentage of the total loaded neutrophils in the PBMC (LDNs) or PMN (HDNs) layer. The dashed lines depict the normal unstimulated situation ( $n=9$, also see Figure $1 A$ ), whereas the solid lines represent the neutrophils of different healthy volunteers after an in vivo LPS challenge $(n=8)$. C. Percentage of CD16 ${ }^{\text {dim }}$ neutrophils in the LDN and HDN fraction and for all neutrophils after LPS challenge $(n=9)$. Data are depicted as median with interquartile range (IQR). Data of $A$ and $C$ were analyzed using Friedman test without correction for multiple comparison. Data of B was analyzed using a Mann Whitney test.

Next, we challenged healthy volunteers with LPS $(2 \mathrm{ng} / \mathrm{kg})$, as a model of controlled acute systemic inflammation as was described before ${ }^{35}$. After LPS more CD16 dim/CD62 Ligh banded neutrophils were recruited to the blood. Significantly more neutrophils appeared on top of the lowest density gradient $(1.077 \mathrm{~g} / \mathrm{ml})$ after LPS compared to the healthy volunteers that is depicted in Figure 1A (Figure 3B). In line with this, more neutrophils appeared on top of the lower Percoll density gradients $(1.079$ and $1.081 \mathrm{~g} / \mathrm{ml})$. After phenotyping the lower and normal density neutrophils, a difference in numbers of CD16 $6^{\text {dim }} / \mathrm{CD}_{2} \mathrm{~L}^{\text {high }}$ neutrophils between the PBMC and the granulocyte fraction after FicollPaque centrifugation (median of $42.0 \%$ in LDNs versus $11.9 \%$ in NDNs) was again apparent (Figure $3 C$ ). Our findings are in line with previous findings in bacterial sepsis where the majority of neutrophils in the PBMC fraction had a banded nucleus ${ }^{32}$. Concluding, our findings indicate that both during homeostasis and acute inflammation, more immature 
(CD16 $\left.{ }^{\mathrm{dim}}\right)$ neutrophils are found in the lower density fraction of neutrophils. This means that not only (early) progenitors (promyelocytes and myelocytes) have a lower density compared to mature neutrophils, but also banded neutrophils.

\section{Are LDNs mainly activated/ degranulated neutrophils?}

As was discussed in section 3, mature neutrophils from healthy individuals decrease their density when activated in vitro, whereas mature neutrophils in inflammatory conditions can already be found in the PBMC fraction at steady state without additional in vitro activation. These inflammatory lower density neutrophils often show signs of activation, especially when compared with neutrophils of healthy donors ${ }^{6,8,22,36}$. Lower density neutrophils from patients with advanced adenocarcinoma in particular displayed higher expression of activation markers like CD11b and CD66b compared to the normal density neutrophils from the same patients ${ }^{37}$. This difference in activation markers between LDNs and NDNs in advanced cancer is remarkably similar to the situation that is present after stimulation of healthy donor neutrophils with $1 \mathrm{nM} \mathrm{fMLF}$ (Figure 2). It is therefore tempting to speculate that during (chronic) inflammation a subset of (mature) neutrophils is more susceptible to activation and that these mature activated neutrophils are the neutrophils found in the PBMC fraction in disease.

It is believed that this activation reflects degranulation in vivo ${ }^{3}$. However, it cannot be excluded that lower density neutrophils are not in vivo activated but rather primed cells that are more susceptible for in vitro manipulation. Arguments for in vivo degranulation are provided by measuring the expression of certain granule markers on the surface of neutrophils, such as CD63 (azurophilic granules), CD66b (specific granules), gelatinase granules (CD11b) and secretory vesicles ${ }^{38,37}$. The extend of degranulation in vivo is poorly understood as the data of the actual content of the different granules and vesicles in neutrophils, e.g. microscopy data, is scarce. Furthermore, existing electron microscopy data of the granular content of LDNs doesn't show signs of significant degranulation ${ }^{39}$. Another argument against degranulation as the cause for the change in buoyant density is the fact that the majority of the in vitro activated neutrophils revert to their original density after only a couple of hours (Supplementary Figure 5) It is quite unlikely for neutrophils to regain granular content in this short time frame. An alternative mechanism could be a change in mean cell volume as a result of water uptake, regulated by aquaporin- $9^{40}$. Changes to a higher forward scatter and a lower side scatter by flow cytometry, which is an argument often used to provide proof of degranulation, could also be caused by this phenomenon ${ }^{24,41}$.

The activated phenotype of circulatory LDNs is perhaps acquired in the tissue. Although, this hasn't been directly shown, neutrophils in the tissue have a very similar phenotype compared to in vitro activated neutrophils ${ }^{42,43}$. This could mean that LDNs are cells which have reverse transmigrated from the endothelial membrane. Moreover, evidence has been provided that homing of neutrophils to the lung tissue is sufficient to adopt an activated phenotype, irrespective of inflammatory disease ${ }^{44}$.

\section{Are LDNs a distinct lineage of cells?}

In systemic lupus erythematosus (SLE) the presence of LDNs has been established by multiple studies ${ }^{25,45,39,46}$. These neutrophils are believed to have a contribution to the pathogenesis of SLE by an enhanced capability to produce type I interferons and 
release neutrophil extracellular traps ${ }^{39,47}$. Moreover, it is suggested that these LDNs have an immature phenotype because of a premature release from the bone marrow, instigated by stimuli which are produced in the course of the disease ${ }^{39}$.

However, other studies have suggested that LDNs in SLE are a complete distinct subset of neutrophils, because they evidently differ from NDNs ${ }^{25,45,39}$. For example, in the study of Singh et al. an increased level of copy number alterations, losses of heterozygosity and microsatellite instability were found in LDNs and not in NDNs ${ }^{45}$. This made the authors suggest that LDNs are an abnormally developed lineage of a neutrophils as a result of genomic damage ${ }^{45}$. Although, this is an interesting model, further research is needed to elucidate these findings. Furthermore, it is still unknown if these findings are specific for SLE or that LDNs from other diseases share these characteristics in genetic variations.

\section{Concluding remarks}

Here we found that circulating neutrophils in healthy individuals are heterogeneous regarding their buoyant density and that low density correlated with the increased responsiveness to $\mathrm{fMLF}$, increased bacterial containment and the capacity to suppress T-cell proliferation. Although it is difficult to compare healthy neutrophils with neutrophils from patients with different inflammatory conditions, it is tempting to speculate that the cells present in the $20 \%$ lowest density fraction of our healthy donors are at least in part comparable with the immunomodulatory cells found in the PBMC fraction in inflammatory conditions such as SLE and cancer. Therefore, we believe that buoyant density is not a distinct feature in a subset of neutrophils found in disease, but rather a spectrum of different densities already found in healthy individuals. An inflammatory condition can subsequently lead to a decrease of the buoyant density of all neutrophils but only the cells on the lower end of the spectrum will shift to the PBMC fraction after Ficoll-Paque density centrifugation (Graphical Abstract).

Neutrophils with immunomodulatory probabilities are accompanied with an intrinsic lower buoyancy in both health and disease. One can only speculate what the underlying mechanism for the spectrum in different buoyant densities is during homeostasis. However, the shift of the spectrum during inflammation can be related to neutrophil maturation stage, activation status and granulation. We do not exclude, however, that separate neutrophil lineages are being recruited and constitute part of the LDN fraction during inflammation. We encourage further studies to focus on mechanistic features that can explain a spectrum in buoyant densities in neutrophils rather than a subdivision of only two subsets (LDNs and NDNs).

\section{Material and Methods}

\section{Human volunteers}

Blood samples were provided by anonymous, healthy volunteers between the age of 18-65 years, male and female, after given written informed consent in accordance to de Declaration of Helsinki. All experiments were performed in accordance with the relevant guidelines and regulations. This study was approved by the University Medical Center Utrecht ethical review committee (METC). 


\section{Experimental endotoxemia model}

The human experimental endotoxemia experiment was induced exactly as described before ${ }^{35}$. In short: a single dose of $2 \mathrm{ng} / \mathrm{kg}$ bodyweight LPS (US Standard Reference Endotoxin Escherichia coli O:113, Lot \#94332B1 obtained from the Pharmaceutical Development Section of the National Institutes of Health, Bethesda, MD) was injected systemically in healthy male and female volunteers with an age between 18 and 30 years old at $\mathrm{t}=0$ hours. At $\mathrm{t}=3$ hours blood was obtained from the same volunteers. The study was approved by the ethics review board of the Radboud University Medical Center and is in compliance with declaration of Helsinki; International Conference on Harmonisation Good Clinical Practice guidelines, and the rulings of the Dutch Medical Research Involving Human Subjects Act. Written informed consent was obtained from all study participants.

\section{Neutrophil isolation}

Human blood samples were collected in sodium heparin tubes (Vacuette ${ }^{\circledR}$ Greiner bio-one, Kremsmünster, Austria). First, whole blood was diluted by adding 1:1 PBS supplemented with $0.32 \% \mathrm{w} / \mathrm{v}$ trisodium citrate (both prepared by the UMCU pharmacy) and $10 \% \mathrm{w} / \mathrm{v}$ human serum albumin (Sanquin, Amsterdam, the Netherlands) (PBS2+), after which it was centrifuged over Ficoll-Paque (Pharmacia, Uppsala, Sweden) for 20 minutes at $760 \times \mathrm{g}$ at $20^{\circ} \mathrm{C}$. The peripheral blood mononuclear cell (PBMC) -layer was harvested to check for neutrophils. Then, the red blood cells in the pellet were lysed for 15-20 minutes in isotonic ice-cold $\mathrm{NH}_{4} \mathrm{Cl}$ solution. Cells were washed twice and After washing the neutrophils were resuspended in PBS2+, and kept on ice until use. Neutrophil counts were measured on an automatic hematology analyzer (CELL-DYN Emerald, Abbott, IL, USA). Isolation of the neutrophil yielded a cell suspension with $>95 \%$ neutrophils in all cases.

\section{Isolation of LDNs after neutrophil stimulation}

Activation agents used for stimulation of the isolated neutrophils were $\mathrm{N}$-formylmethionine-leucyl-phenylalaline (fMLF) (Sigma-Aldrich, St. Louis, USA) and platelet activation factor-16 (PAF) (Calbiochem, EMD Chemicals, Inc., San Diego, CA, USA). For the purpose of the experiments described below each reagent was diluted to $10 \mu \mathrm{M}-1 \mathrm{pM}$. Neutrophils were stimulated for 15 minutes in a water bath at $37^{\circ} \mathrm{C}$. Hereafter, the neutrophils were centrifuged over Ficoll-Paque as described above. The neutrophils that shifted to the 'PBMC' layer were regarded as LDNs, the neutrophils which remained below were regarded as HDNs.

\section{Isolation of LDNs without neutrophil stimulation}

Percoll with different densities was prepared for this experiments by diluting Percoll with a density of $1.128 \mathrm{~g} / \mathrm{ml}$ (MP Biomedicals, LLC, Solon Ohio) with 10xPBS, PBS, $0.32 \%$ $\mathrm{w} / \mathrm{v}$ trisodium citrate (prepared by the UMCU pharmacy) and $10 \% \mathrm{w} / \mathrm{v}$ human serum albumin solution (Sanquin, Amsterdam, the Netherlands) in such a way that it yielded different calculated densities: 1.079, 1.081 and $1.083 \mathrm{~g} / \mathrm{ml}$. Osmolarity and $\mathrm{pH}$ were corrected accordingly to $290 \mathrm{mOsm} / \mathrm{kgH}_{2} \mathrm{O}$ and 7.3 , respectively. Granulocytes that were previously isolated with Ficoll-Paque were, thereafter, centrifuged on different densities of Percoll for 20 minutes at $760 \times \mathrm{g}$ at room temperature. Approximately $10-$ 
$20 \%$ of the lowest density neutrophils, typically found on top of Percoll gradient with a density of $1.081 \mathrm{~g} / \mathrm{ml}$ and approximately $10-20 \%$ of the highest density neutrophils, typically found below the Percoll gradient with a density of $1.083 \mathrm{~g} / \mathrm{ml}$ were used for further experiments. Comparable with the density shifts after stimulation, these cells were similarly named lower density (LDNs) and higher density neutrophils (HDNs) respectively.

\section{Containment assay}

A neutrophil containment assay was used modified from Li et al. ${ }^{48}$ and described before by van Grinsven et $\mathrm{al}^{15}$. In short, a gel with fibrin scaffolds was formed to mimic a tissue-like environment. $100 \mathrm{uL}$ of the fibrin gel was made by mixing $50 \mu \mathrm{L}$ of $5 \times 10^{5}$ neutrophils/ml in Hepes buffer with $50 \mu \mathrm{L}$ Hepes buffer containing $2 \mathrm{mg} / \mathrm{ml}$ fibrinogen (Sigma-Aldrich) with $40 \%$ human pooled serum (Sigma-Aldrich), $1 \mathrm{U} / \mathrm{ml}$ thrombin (Sigma-Aldrich) and $5 \times 10^{5}$ CFU GFP-expressing Staphylococcus aureus. This mix was resuspended before solidification in a 96 well clear bottom polysterene plate (Corning Costar, New York, NY, USA). Neutrophil survival was determined by adding propidium iodide (PI) to separate wells containing neutrophils. The plates were then incubated in $37^{\circ} \mathrm{C}$ and the fluorescence of the GFP or PI in single wells was measured every 20 minutes in the Fluostar Optima or Omega plate reader (BMG technologies, Ortenberg, Germany). Raw data from the experiments were exported to Microsoft Excel and the lag time was determined as described before ${ }^{15}$.

\section{Lymphocyte proliferation suppression assay}

Unstimulated lymphocytes were isolated from the PBMC fraction after centrifugation over Ficoll-Paque. Cells were then stained with 5(6)-Carboxyfluorescein diacetate $\mathrm{N}$-succinimidyl ester (CFSE) (Sigma-Aldrich, St. Louis, USA) and washed twice with RPMI 1640 (Gibco, Introgen, Breda, the Netherlands). Thereafter, cells were cultured for 96 hours in IMDM (Gibco, Introgen, Breda, the Netherlands) supplemented with $5 \%$ FCS and $1 \%$ penicillin/streptomycin (both from Gibco, Introgen, Breda, the Netherlands). 100.000 lymphocytes were activated with anti- $0.15 \mu \mathrm{g} / \mathrm{ml}$ CD3 and 0.2 $\mu \mathrm{g} / \mathrm{ml}$ CD28 (both from Sanquin, Amsterdam, the Netherlands) and incubated with either 200.000 LDNs, HDNs or total neutrophils (as is explained above). After 96 hours proliferation of lymphocytes was assessed by determining CFSE dilution on a BD Canto $\mathrm{II}^{\mathrm{TM}}$ cell analyzer (Becton Dickinson, Mountain view CA, USA). Flow cytometry data was analyzed using the FlowJo ${ }^{\circledR}$ v10 software (FlowJo, LLC, Ashland, OR, USA). The division index, proliferation index and percentage of divided lymphocytes were automatically calculated by an algorithm incorporated in FlowJo.

\section{Phagocytosis assay}

Isolated LDNs and HDNs, and total neutrophils (as a control) were isolated as described above. After isolation the neutrophils were stained with CD45-APC (clone 2D1; BD, San Jose, CA, USA) for HDNs and CD45-PE-Cy7 (clone HI30; BD) for LDNs on ice for 20 minutes in the dark. Hereafter, the cells were washed once and resuspended in $100 \mathrm{uL}$ supplemented Hepes buffer.

Neutrophil suspension with either LDNs, HDNs or a mix with both neutrophil populations in concentration of $5 \times 10^{6}$ neutrophils/mL was prepared in Hepes buffer supplemented 
with $40 \%$ human serum. GFP-expressing Staphylococcus aureus was then added with a multiplicity of infection of 1 or 2 . After gentle resuspension of the mix, it was placed in a shaking incubator (Innova ${ }^{\circledR} 44$, New Brunswick Scientific, Edison, NJ, USA) at $37^{\circ} \mathrm{C}$ and $180 \mathrm{rpm}$. After 40 minutes samples were taken from the suspension and fixed with 1\%PFA (in PBS) on ice.

\section{Flowcytometry analysis}

Cells were analyzed using BD LSRFortessa ${ }^{\mathrm{TM}}$ cell analyzer (Becton Dickinson, Mountain view CA, USA) for multi-color experiments or BD Canto $\mathrm{II}^{\mathrm{TM}}$ cell analyzer (Becton Dickinson) for the two- four color experiments. One million cells were stained with antibodies for 30 minutes in a concentration of 40 million cells per $\mathrm{ml}$ on ice. Cells were washed twice with PBS2+ before analysis. The following antibodies were used: CD35-PE/ clone E11; CD62L-PE-Cy7/clone Dreg56; CD66b-AF647/clone G10F5 (all from Biolegend, San Diego, CA, USA); CD11b-APC-Cy7/clone ICRF44 and CD16-PB/clone $3 G 8$ $(B D$, San Jose, $C A$, USA). Neutrophils were differentiated from remaining contaminating cells according to specific scatter patterns on forward and side scatter, CD66b and on CD16 expression (see Supplementary Figure 6). $\mathrm{Ca}^{2+}$ influx experiments were conducted by loading 1 million cells with $1 \mu \mathrm{M}$ Fluo-4, AM (Thermo Fisher, Massachusetts, USA) for 30 minutes in $37^{\circ} \mathrm{C}$. In the phagocytosis assay neutrophils of different densities were distinguished based on their respective CD45 staining. Banded cells were identified as CD16 ${ }^{\text {dim }}$ and $C D 62 L^{\text {high }}$ (Supplementary Figure 6). For all experiments at least 10.000 events were recorded for data analysis.

\section{Statistical analysis}

Data was plotted and statistical analysis was preformed using GraphPad Prism (GraphPad Software, La Jolla, CA, USA). Results are expressed by median \pm interquartile range or mean $\pm \mathrm{SE}$. A Mann-Whitney test or a Wilcoxon test was used when only two groups were compared. When comparing more than two groups a Friedman with uncorrected Dunn's test was performed. Statistical significance was defined as $p<0.05$.

\section{Acknowledgments}

The authors thank Erinke van Grinsven, who developed the lymphocyte proliferation suppression assay. 


\section{References}

1. Ng LG, Ostuni R, Hidalgo A. Heterogeneity of neutrophils. Nat Rev Immunol. 2019;19:255265.

2. Hacbarth E, Kajdacsy-Balla A. Low density neutrophils in patients with systemic lupus erythematosus, rheumatoid arthritis, and acute rheumatic fever. Arthritis Rheum. 1986;29:1334-42.

3. Scapini P, Marini O, Tecchio C, et al. Human neutrophils in the saga of cellular heterogeneity: insights and open questions. Immunol Rev. 2016;273:48-60.

4. Pember SO, Barnes KC, Brandt SJ, et al. Density heterogeneity of neutrophilic polymorphonuclear leukocytes: gradient fractionation and relationship to chemotactic stimulation. Blood. 1983;61:1105-15.

5. Pember SO, Kinkade JM. Differences in myeloperoxidase activity from neutrophilic polymorphonuclear leukocytes of differing density: relationship to selective exocytosis of distinct forms of the enzyme. Blood. 1983;61:1116-24.

6. Miyagawa $\mathrm{H}$, Okada $\mathrm{C}$, Sugiyama $\mathrm{H}$, et al. Density distribution and density conversion of neutrophils in allergic subjects. Int Arch Allergy Appl Immunol. 1990;93:8-13.

7. Herteman N, Vargas A, Lavoie J-P. Characterization of Circulating Low-Density Neutrophils Intrinsic Properties in Healthy and Asthmatic Horses. Sci Rep. 2017;7:7743.

8. Ssemaganda A, Kindinger $L$, Bergin $P$, et al. Characterization of neutrophil subsets in healthy human pregnancies. PLoS One. 2014;9:e85696.

9. Fu J, Tobin MC, Thomas LL. Neutrophil-like low-density granulocytes are elevated in patients with moderate to severe persistent asthma. Ann Allergy Asthma Immunol. 2014;113:635640.e2.

10. Cloke T, Munder M, Taylor G, et al. Characterization of a novel population of low-density granulocytes associated with disease severity in HIV-1 infection. PLoS One. 2012;7:e48939.

11. Hellebrekers P, Vrisekoop N, Koenderman L. Neutrophil phenotypes in health and disease. Eur J Clin Invest. 2018;48:e12943.

12. Eggleton $P$, Fisher D, Crawford N. Heterogeneity in the circulating neutrophil pool: studies on subpopulations separated by continuous flow electrophoresis. J Leukoc Biol. 1992;51:61725.

13. Eggleton P, Wang L, Penhallow J, et al. Differences in oxidative response of subpopulations of neutrophils from healthy subjects and patients with rheumatoid arthritis. Ann Rheum Dis. 1995;54:916-923.

14. Wenisch C, Patruta S, Daxböck F, et al. Effect of age on human neutrophil function. J Leukoc Biol. 2000;67:40-45.

15. van Grinsven E, Leliefeld PHC, Pillay J, et al. A comprehensive three-dimensional assay to assess neutrophil defense against bacteria. J Immunol Methods. 2018;462:83-90.

16. Pillay J, Tak T, Kamp VM, et al. Immune suppression by neutrophils and granulocytic myeloidderived suppressor cells: similarities and differences. Cell Mol Life Sci. 2013;70:3813-27.

17. Marini $\mathrm{O}$, Costa $\mathrm{S}$, Bevilacqua $\mathrm{D}$, et al. Mature $\mathrm{CD} 10^{+}$and immature $\mathrm{CD} 10$ - neutrophils present in G-CSF-treated donors display opposite effects on T cells. Blood. 2017;129:13431356.

18. Bowers NL, Helton ES, Huijbregts RPH, et al. Immune Suppression by Neutrophils in HIV-1 Infection: Role of PD-L1/PD-1 Pathway. PLoS Pathog. 2014;10:e1003993. 
19. Brandau S, Trellakis S, Bruderek K, et al. Myeloid-derived suppressor cells in the peripheral blood of cancer patients contain a subset of immature neutrophils with impaired migratory properties. J Leukoc Biol. 2011;89:311-317.

20. Rodriguez PC, Ernstoff MS, Hernandez C, et al. Arginase I-Producing Myeloid-Derived Suppressor Cells in Renal Cell Carcinoma Are a Subpopulation of Activated Granulocytes. Cancer Res. 2009;69:1553-1560.

21. Darcy CJ, Minigo G, Piera KA, et al. Neutrophils with myeloid derived suppressor function deplete arginine and constrain T cell function in septic shock patients. Crit Care. 2014;18:R163.

22. Deng $Y$, Ye J, Luo Q, et al. Low-Density Granulocytes Are Elevated in Mycobacterial Infection and Associated with the Severity of Tuberculosis. PLoS One. 2016;11:e0153567.

23. McKenna KC, Beatty KM, Vicetti Miguel $R$, et al. Delayed processing of blood increases the frequency of activated CD11b+CD15+ granulocytes which inhibit T cell function. J Immunol Methods. 2009;341:68-75.

24. Bryk JA, Popovic PJ, Zenati MS, et al. Nature of myeloid cells expressing arginase 1 in peripheral blood after trauma. J Trauma. 2010;68:843-52.

25. Denny MF, Yalavarthi S, Zhao W, et al. A Distinct Subset of Proinflammatory Neutrophils Isolated from Patients with Systemic Lupus Erythematosus Induces Vascular Damage and Synthesizes Type I IFNs. J Immunol. 2010;184:3284-3297.

26. Olofsson T, Gärtner I, Olsson I. Separation of human bone marrow cells in density gradients of polyvinylpyrrolidone coated silica gel (Percoll). Scand J Haematol. 1980;24:254-62.

27. Cowland JB, Borregaard N. Isolation of neutrophil precursors from bone marrow for biochemical and transcriptional analysis. J Immunol Methods. 1999;232:191-200.

28. Ohtsu S, Yagi $\mathrm{H}$, Nakamura $\mathrm{M}$, et al. Enhanced neutrophilic granulopoiesis in rheumatoid arthritis. Involvement of neutrophils in disease progression. J Rheumatol. 2000;27:1341-51.

29. Manz MG, Boettcher S. Emergency granulopoiesis. Nat Rev Immunol. 2014;14:302-314.

30. Honda T, Uehara T, Matsumoto $G$, et al. Neutrophil left shift and white blood cell count as markers of bacterial infection. Clin Chim Acta. 2016;457:46-53.

31. Pillay J, Ramakers BP, Kamp VM, et al. Functional heterogeneity and differential priming of circulating neutrophils in human experimental endotoxemia. J Leukoc Biol. 2010;88:211220.

32. Drifte G, Dunn-Siegrist I, Tissières $P$, et al. Innate Immune Functions of Immature Neutrophils in Patients With Sepsis and Severe Systemic Inflammatory Response Syndrome*. Crit Care Med. 2013;41:820-832.

33. van den Akker ELT, Baan CC, van den Berg B, et al. Ficoll-separated mononuclear cells from sepsis patients are contaminated with granulocytes. Intensive Care Med. 2008;34:912-916.

34. Pillay J, Kamp VM, van Hoffen $E$, et al. A subset of neutrophils in human systemic inflammation inhibits T cell responses through Mac-1. J Clin Invest. 2012;122:327-36.

35. van Lier D, Geven C, Leijte GP, et al. Experimental human endotoxemia as a model of systemic inflammation. Biochimie. Epub ahead of print June 22, 2018. DOI: 10.1016/J. BIOCHI.2018.06.014.

36. Sippel TR, White J, Nag K, et al. Neutrophil Degranulation and Immunosuppression in Patients with GBM: Restoration of Cellular Immune Function by Targeting Arginase I. Clin Cancer Res. 2011;17:6992-7002.

37. Liu Y, Hu Y, Gu F, et al. Phenotypic and clinical characterization of low density neutrophils in patients with advanced lung adenocarcinoma. Oncotarget. 2017;8:90969-90978. 
38. Jog NR, Rane MJ, Lominadze G, et al. The actin cytoskeleton regulates exocytosis of all neutrophil granule subsets. Am J Physiol Physiol. 2007;292:C1690-C1700.

39. Carmona-Rivera C, Kaplan MJ. Low-density granulocytes: a distinct class of neutrophils in systemic autoimmunity. Semin Immunopathol. 2013;35:455-463.

40. Karlsson T, Glogauer M, Ellen RP, et al. Aquaporin 9 phosphorylation mediates membrane localization and neutrophil polarization. J Leukoc Biol. 2011;90:963-973.

41. Lee A-J, Kim S-G. Mean cell volumes of neutrophils and monocytes are promising markers of sepsis in elderly patients. Blood Res. 2013;48:193-7.

42. Buckley $C D$, Ross EA, McGettrick HM, et al. Identification of a phenotypically and functionally distinct population of long-lived neutrophils in a model of reverse endothelial migration. $J$ Leukoc Biol. 2006;79:303-311.

43. Watson F, Robinson JJ, Phelan M, et al. Receptor expression in synovial fluid neutrophils from patients with rheumatoid arthritis. Ann Rheum Dis. 1993;52:354-359.

44. Fortunati E, Kazemier KM, Grutters JC, et al. Human neutrophils switch to an activated phenotype after homing to the lung irrespective of inflammatory disease. Clin Exp Immunol. 2009;155:559-566.

45. Singh N, Traisak P, Martin KA, et al. Genomic alterations in abnormal neutrophils isolated from adult patients with systemic lupus erythematosus. Arthritis Res Ther. 2014;16:R165.

46. van den Hoogen LL, van der Linden M, Meyaard L, et al. Neutrophil extracellular traps and low-density granulocytes are associated with the interferon signature in systemic lupus erythematosus, but not in antiphospholipid syndrome. Ann Rheum Dis. 2019; annrheumdis-2019-215781.

47. van der Linden M, van den Hoogen LL, Westerlaken GHA, et al. Neutrophil extracellular trap release is associated with antinuclear antibodies in systemic lupus erythematosus and antiphospholipid syndrome. Rheumatology. 2018;57:1228-1234.

48. Li Y, Loike JD, Ember JA, et al. The Bacterial Peptide $N$-Formyl-Met-Leu-Phe Inhibits Killing of Staphylococcus epidermidis by Human Neutrophils in Fibrin Gels. J Immunol. 2002;168:816824. 


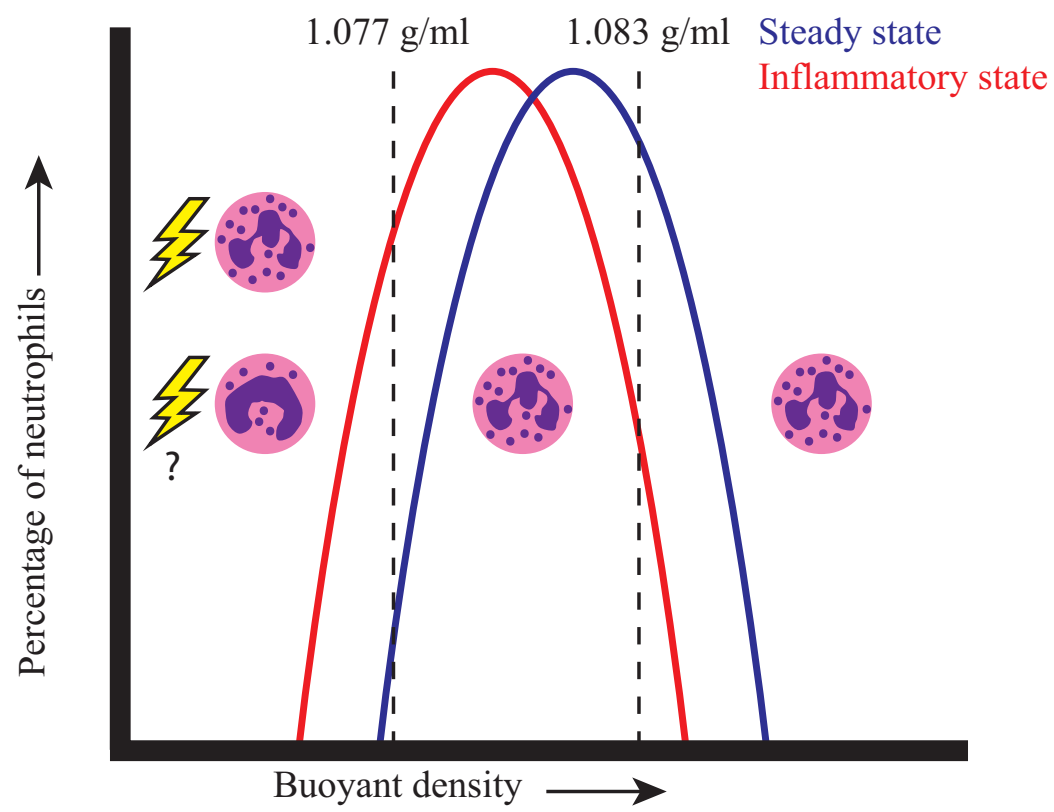

Graphical abstract. Proposed model for neutrophil buoyant density.

Neutrophil density is normally distributed during homeostasis and disease. After activation of neutrophils (in vitro or in vivo) all neutrophil densities shift to the left. In diseases with (chronic) inflammation more LDNs are found in the blood because of i) the appearance of immature neutrophils and ii) because mature neutrophils change in density after degranulation/ activation.

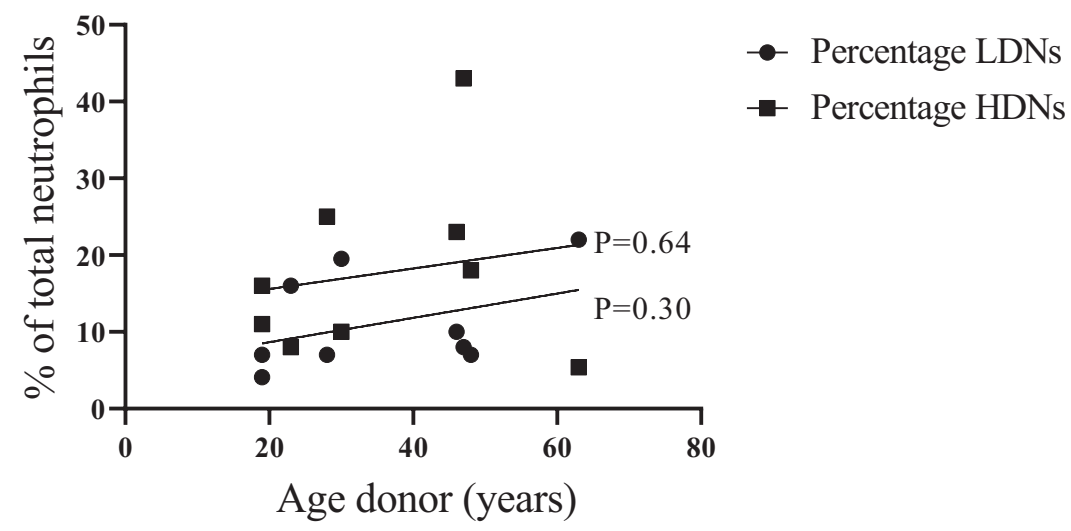

Supplementary Figure 1. Correlation between age and percentage of LDNs and HDNs.

The correlation between the donors age and the percentage of LDNs (circles and grey line) and HDNs (squares and black line) is depicted $(n=9)$. A linear regression was performed to test for significance. 
A

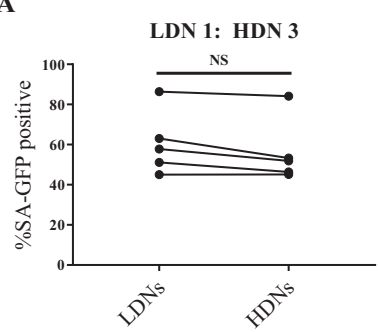

D

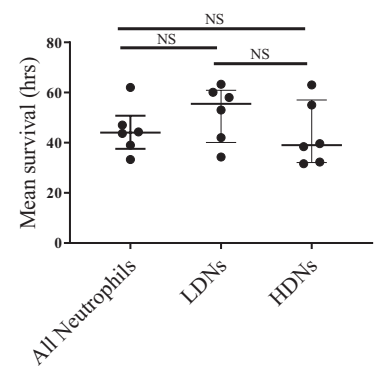

B

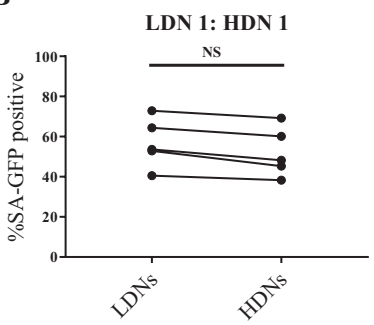

C

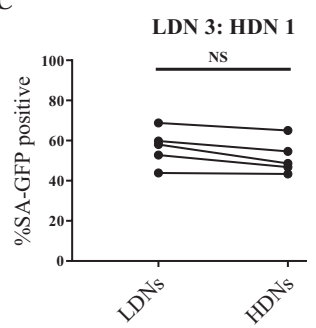

Supplementary Figure 2. Differences in phagocytosis and survival of unstimulated LDNs and HDNs.

A-C Data is depicted as percentage of GFP positive neutrophils and their mean fluorescence intensity $(\mathrm{MFI}, \mathrm{n}=5)$. Different ratios of LDNs/HDNs were titrated in suspension with S. aureus with a MOI of 2. D. Mean survival of all neutrophils, $\operatorname{HDNs}$ or $\operatorname{LDNs}(n=6)$. Data are depicted as median with interquartile range (IQR). Data were analyzed using a Wilcoxon test.
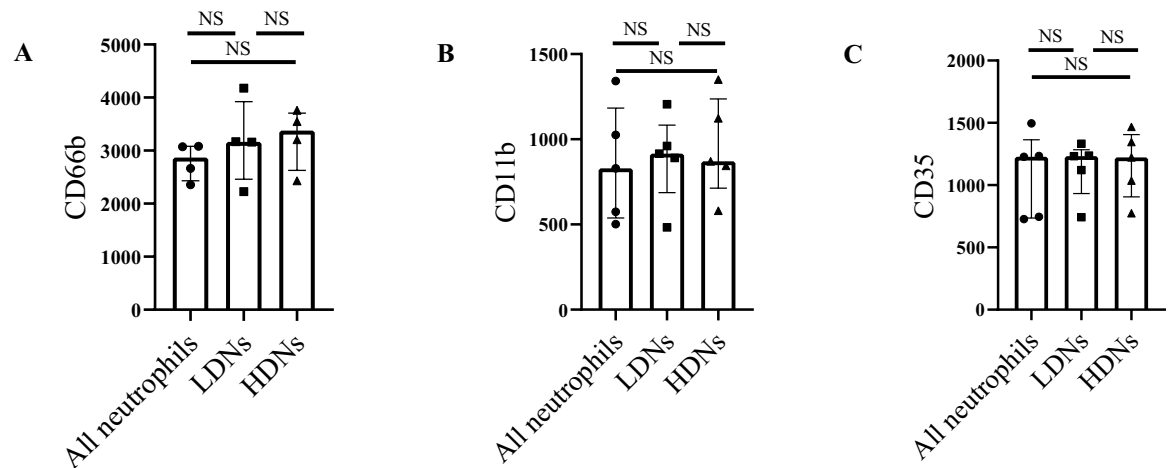

Supplementary Figure 3. Activation markers on unstimulated LDNs and HDNs.

A. CD66b ( $n=4)$. B. CD11b $(n=5)$. C. CD35 $(n=5)$. Data are depicted as median with interquartile range (IQR). Data were analyzed using Friedman test without correction for multiple comparisons. 
A

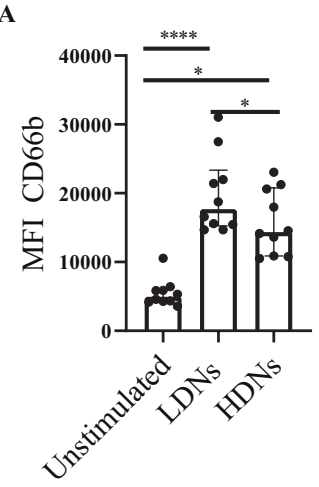

D

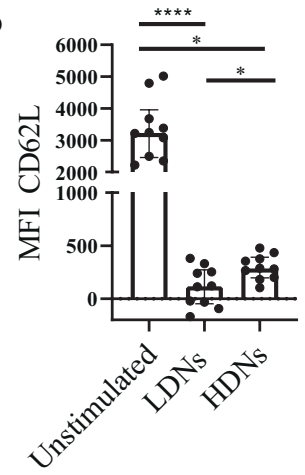

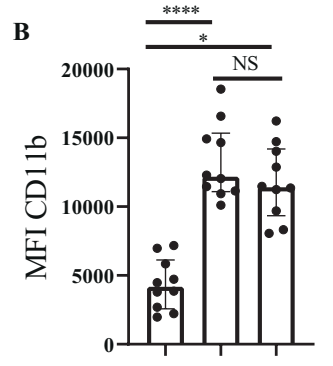
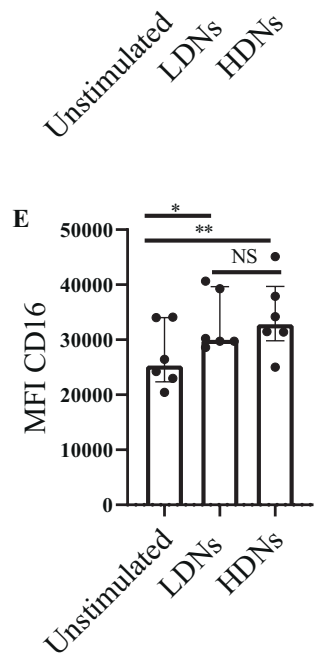

C
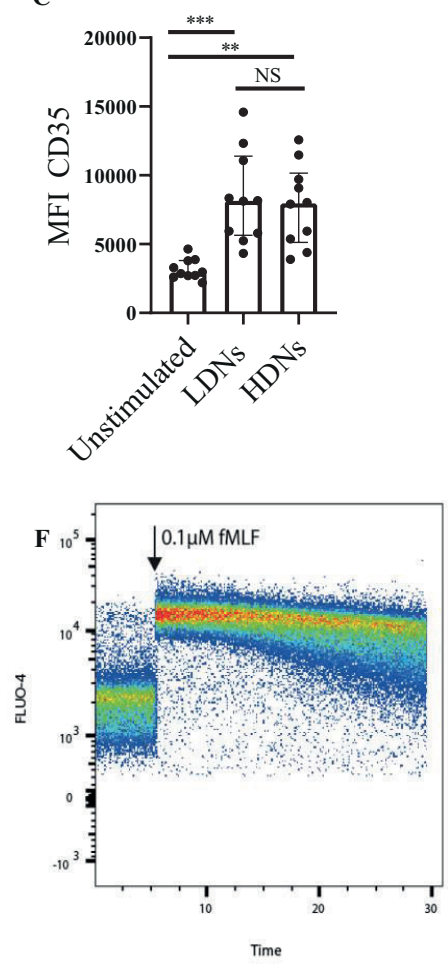

Supplementary Figure 4. Median fluorescence intensity of membrane activation markers after $0.1 \mu \mathrm{M}$ fMLF.

A. $\operatorname{CD} 66 b(n=6)$ B. $\operatorname{CD} 11 b(n=6)$ C. $\operatorname{CD} 35(n=6)$. D. CD62L $(n=6)$ E. CD16 (n=6). Data are depicted as median with interquartile range (IQR). Data were analyzed using Friedman test without correction for multiple comparison. F. Healthy donor neutrophils were stained with Fluo-4 to measure the $\mathrm{Ca}^{2+}$ influx after the addition of $0.1 \mu \mathrm{M}$ fMLF. 
A

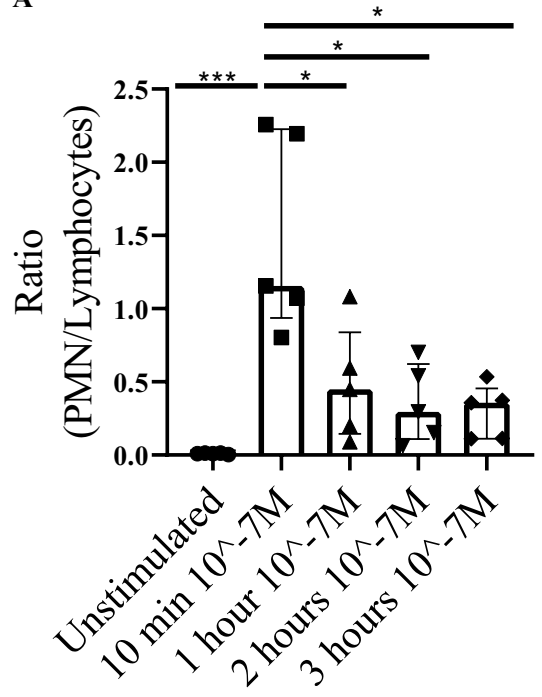

B

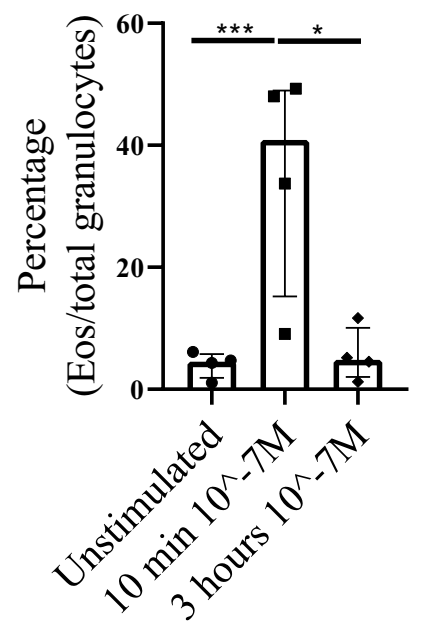

Supplementary Figure 5. Neutrophil density after $10^{-7} \mathrm{M}$ fMLF stimulation in time.

A. Depicted is the ratio of the percentage of granulocytes and lymphocytes in the PBMC fraction over time after incubation with $\mathrm{fMLF}\left(10^{-7} \mathrm{M}, \mathrm{n}=5\right)$ for 10 minutes, 1 hour, 2 hours and 3 hours. B. Percentage of eosinophils in the normal density fraction is depicted for unstimulated blood or for 10 minutes or 3 hours stimulated blood (with $10^{-7} \mathrm{M} \mathrm{fMLF}$ ). Data are depicted as median with interquartile range (IQR). Data were analyzed using Friedman test without correction for multiple comparison. All conditions were tested, but only statistically significant results are indicated. 
A

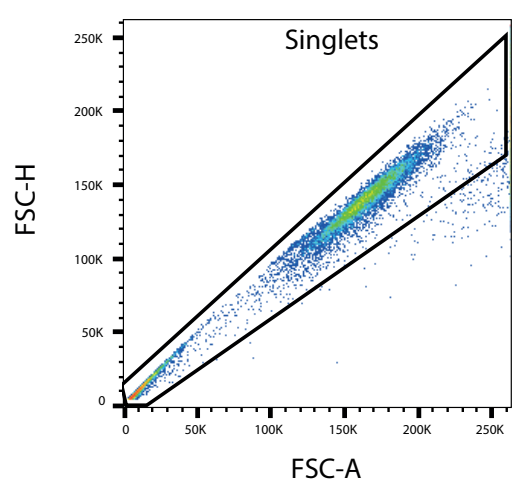

C

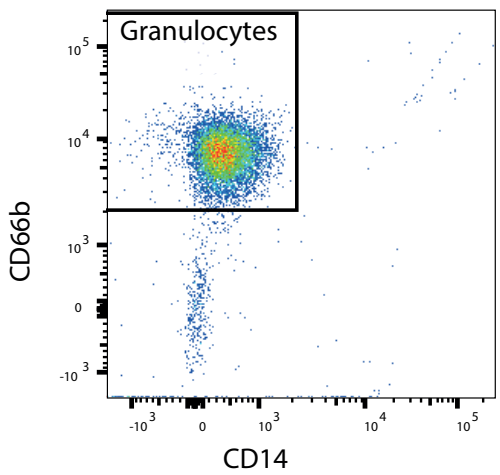

B

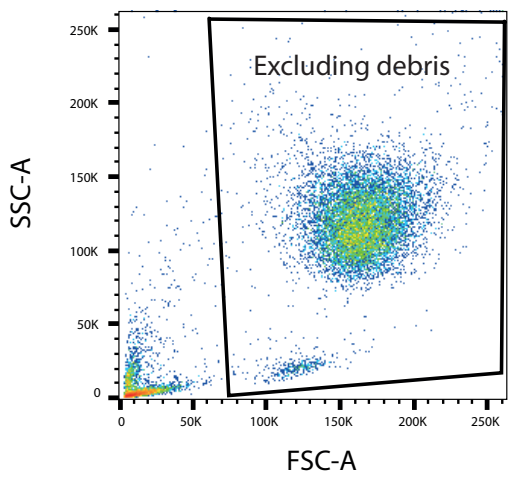

D

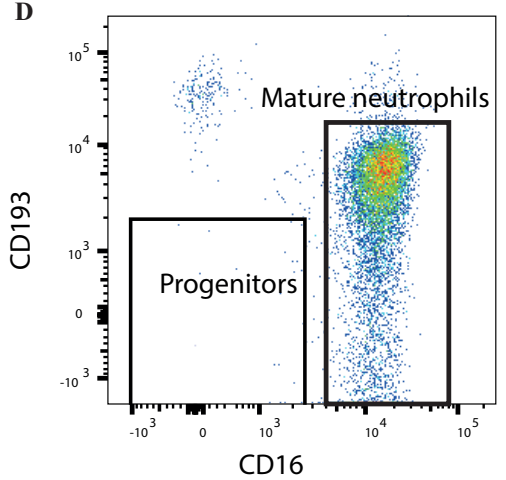

$\mathbf{E}$
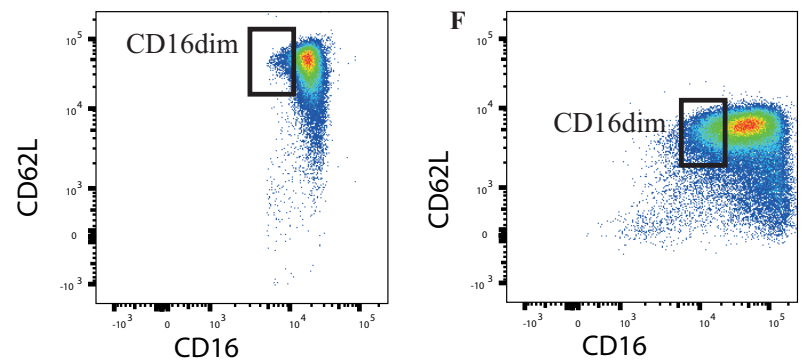

Supplementary Figure 6. Gating strategy for neutrophils.

Neutrophils from the different experimental setups were gated as follows. A. doublets were gated out using FSC-A and FSC-H. B. Next, debris was gated out on the basis of FSC-A and SSC-A. C. Then granulocytes were recognized and gated as $\mathrm{CD}_{6} 6 \mathrm{~b}^{+}$and $\mathrm{CD} 14^{\text {low }}$. D. Mature neutrophils were gated as CD16 ${ }^{\text {high }}$. E. Neutrophils with a banded nucleus were recognized as CD16 dim and CD62 ${ }^{\text {high }}$ in healthy volunteers and F. after LPS administration. 



\section{Chapter 4}

\section{Mepolizumab does not affect neutrophil kinetics in eosinophilic asthma}

M. Hassani ${ }^{1,2}$, T. Tak ${ }^{1,2}$, C. van Aalst ${ }^{1,2}$, S. Van Nederveen ${ }^{3}$, K. Tesselaar ${ }^{1}$, J.A.M. Borghans $^{1}$, N. Vrisekoop ${ }^{1,2}$ and L. Koenderman ${ }^{1,2}$.

1. Center for Translational Immunology, University Medical Center Utrecht, Utrecht, The Netherlands.

2. Department of Respiratory Medicine, University Medical Center Utrecht, Utrecht, The Netherlands.

3. Department of Respiratory Medicine, HAGA hospital, The Hague, The Netherlands. 


\section{Abstract}

Eosinophilic asthma is characterized by the presence of large number of eosinophils $(>3 \%)$ in the bronchial airways. However, even in the case of eosinophilic asthma, neutrophils are often also recruited to the airways. Still, the reason for their recruitment in eosinophilic asthma is largely unknown.

We hypothesized that neutrophils are recruited to the bronchial airways as a response to damage caused by type 2 inflammation (DAMPS). So, according to our hypothesis, treatment with anti-IL-5, which largely abolishes the presence of eosinophils in the airways, would also lead to fewer neutrophils in the sputum and/or difference in neutrophil kinetics.

Therefore, we included patients with moderate to severe eosinophilic asthma who were treated with mepolizumab $(n=10)$ or placebo $(n=10)$. The kinetics of neutrophils were studied after short term treatment with ( $1^{\text {st }}$ phase $)$ and after 84 days of treatment with mepolizumab or placebo $\left(2^{\text {nd }}\right.$ phase $)$ by labelling patients twice with 6,6- ${ }^{2} \mathrm{H}_{2}$-glucose (deuteriated glucose) for 6 hours. After labelling, blood and sputum was collected at different time points and neutrophils were isolated using FACS sorting. DNA ${ }^{2} \mathrm{H}$-enrichment was determined by GC-MS.

We did not find any change in the absolute number and kinetics of blood and sputum neutrophils in patients with eosinophilic asthma after treatment with Mepolizumab (anti-IL-5). This suggests that tissue damage present in eosinophilic asthma does not lead to a considerable change in neutrophil kinetics. On the other hand, three different blood neutrophil populations with distinct kinetics were found in the circulation of these patients. $\mathrm{CD} 16^{\text {neg }} \mathrm{CD} 62 \mathrm{~L}^{\text {neg }}$ were found to be most likely neutrophil progenitors, CD16 ${ }^{\text {high }} \mathrm{CD} 62 \mathrm{~L}^{\text {high }}$ are most likely normal segmented neutrophils and CD16 ${ }^{\text {high }}$ CD62 $\mathrm{L}^{\text {dim }}$ neutrophils are hypersegmented neutrophils. The hypersegmented neutrophils have one day delay in label enrichment compared to the normal segmented neutrophils, which makes them most likely more mature. 


\section{Introduction}

Nowadays, asthma is recognized as a collection of heterogeneous respiratory conditions with distinct endotypes, each considered to exhibit a different pathophysiological mechanism $^{1}$. One of the described endotypes is eosinophilic asthma which is characterized by a dominant eosinophilic bronchial inflammation. Eosinophilic airway inflammation is defined as the presence of eosinophils in the induced sputum with a cut-off value of $3 \%^{2}$. However, in daily practice blood eosinophil counts seem more reliable and useful alternative for sputum inductions, although an explicit cut-off point for eosinophilia is lacking (ranging from 150-450 cells/ $\mu \mathrm{L})^{3,4}$. In eosinophilic asthma, neutrophils are also present in sputum in high numbers (up to $61 \%$ of total cells) 5 . Furthermore, we have shown that neutrophils have an activated phenotype regardless of the number in the sputum or the studied phenotype ${ }^{6}$. Besides, neutrophils most likely play a large role in exacerbations triggered by viral infections, also in eosinophilic asthma ${ }^{7}$. Consequently, in eosinophilic asthma, it is possible that neutrophils are recruited as a response to eosinophilic inflammation. Since, neutrophils are known to be recruited as a response to damage associated molecular patterns (DAMPs) ${ }^{8}$, it is likely that damage caused by type 2 or eosinophilic inflammation can secondary lead to neutrophil recruitment. Therefore, we wondered whether eosinophil targeted therapy by anti-IL-5 antibodies would indirectly affect neutrophil kinetics in patients with eosinophilic asthma.

Hence we studied the kinetics of neutrophils in the blood and sputum of eosinophilic asthma patients, using an vivo labelling method ${ }^{9}$. This method makes use of the fact that ${ }^{2} \mathrm{H}_{2}-6,6$-glucose is built into the ribose moiety of the DNA of dividing cells, including cells within the mitotic pool of neutrophils progenitors. Afterwards, a pulsechase experiment is conducted by following the enrichment of ${ }^{2} \mathrm{H}$ in DNA of neutrophils at different time points.

\section{Materials and Methods}

\section{Subjects}

20 patients with moderate to severe eosinophilic asthma (EA) were enrolled at the respiratory medicine department of the University Medical Center in Utrecht and of the HAGA Hospital in the Hague (ABR NL57535.041.16). All participants gave a written informed consent in accordance to the Declaration of Helsinki (Forteleza, 2013). The study was approved by the local medical ethical committee (IRB).

All participants were between the age of 18-70 years and were diagnosed with asthma according to GINA guidelines. Moreover, patients had at least one blood eosinophil number of $0.25 \times 10^{6} / \mathrm{ml}$ in the past year prior to enrollment. For the complete inclusion and exclusion criteria see chapter 9 of this thesis. Because, the sputum samples lacked data points between day 8 and 13 after label intake, we included 9 additional eosinophilic asthma patients from an earlier study (EHLA, ABR NL44779.041.13). These 9 patients had similar inclusion and exclusion criteria, but were not randomized for mepolizumab or placebo (table 1). 


\begin{tabular}{|l|c|c|c|}
\hline Patient characteristics & Placebo & Mepolizumab & EHLA \\
\hline $\mathbf{N}$ & 10 & 10 & 9 \\
\hline Female (\%) & $5(50 \%)$ & $6(60 \%)$ & $4(44 \%)$ \\
\hline $\begin{array}{l}\text { Age at inclusion, } \\
\text { years (IQR) }\end{array}$ & $51(33-65)$ & $57(46-62)$ & $56(40-72)$ \\
\hline BMI, kg/m² (IQR) & $26.2(23.5-34.0)$ & $28.9(24.6-35.1)$ & $27.2(23.2-32.2)$ \\
\hline $\begin{array}{l}\text { Blood eosinophil count at } \\
\text { inclusion 106/ml, (IQR) }\end{array}$ & $0.49(0.28-0.80)$ & $0.40(0.22-0.70)$ & $0.54(0.10-2.2)$ \\
\hline $\begin{array}{l}\text { Blood neutrophil count at } \\
\text { inclusion 10\%/ml, (IQR) }\end{array}$ & $3.45(3.01-4.07)$ & $3.45(3.00-3.51)$ & $4.60(2.60-9.40)$ \\
\hline
\end{tabular}

Table 1. Patient characteristics in the placebo and mepolizumab group.

Data represent median values (interquartile range).

\section{Deuterium labelling procedure and randomization of subjects for mepolizumab or placebo}

Patients were labelled with 12 half-hourly doses of $6,6-{ }^{2} \mathrm{H}_{2}$-glucose amounting to a total of $1 \mathrm{~g} / \mathrm{kg}$ bodyweight (Cambridge Isotope Laboratories, Tewksbury, MA, USA). Patients were blindly randomized and were treated with $100 \mathrm{mg}$ mepolizumab or placebo every 4 weeks for a total of 4 administrations (see Table 1 for baseline characteristics). The first administration of placebo or mepolizumab was four days after the first label intake. The labelling procedure was performed twice per patient: at the start of the study $(t=$ 0 days, phase 1 ) and after 84 days ( $t=0$ days, phase 2 ), see Figure 1 . A total of ten blood samples was taken from all patients during the course of the study, of which two are directly before the start of both labelling procedures (Figure 1). Furthermore, three sputum samples per patient were obtained. For blood and sputum, the time points varied between volunteers, but were largely similar between the mepolizumab and placebo group to maximize the total number of data points for each group. 
A

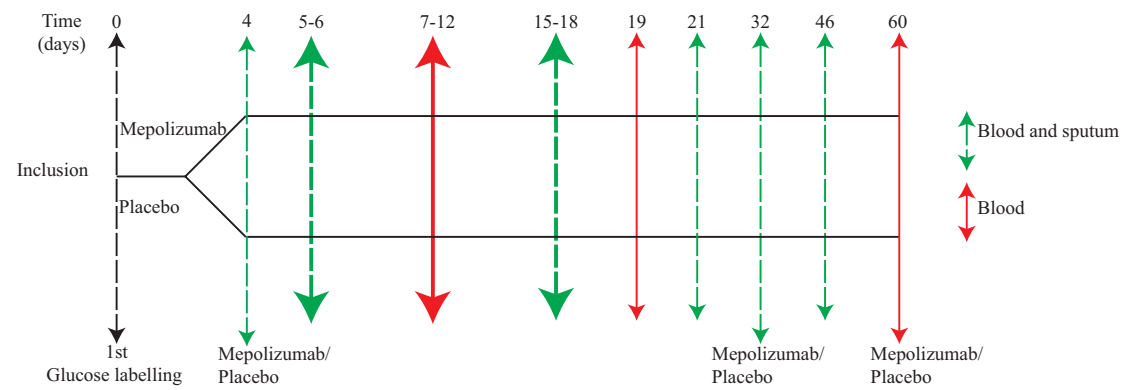

2nd phase

B

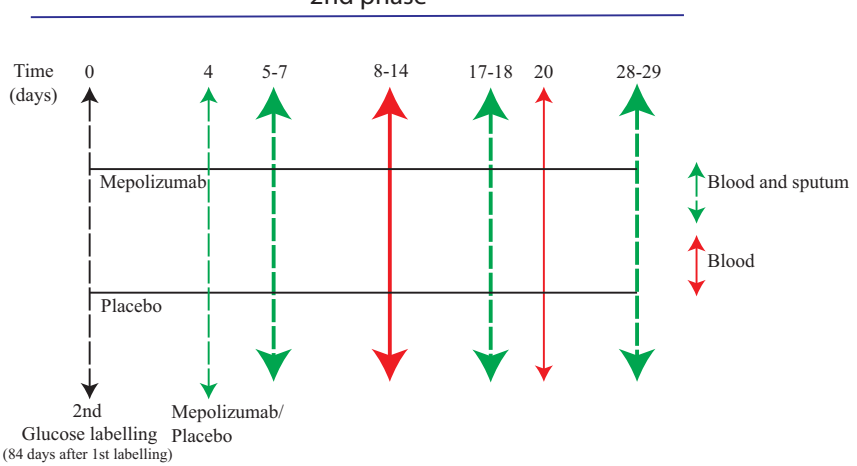

Figure 1. Flowchart of study design,

A. During the $1^{\text {st }}$ phase of the study 20 asthma patients were enrolled. At day 0 , all patients were labelled with deuterium glucose and baseline blood sample was withdrawn. Thereafter patients were blindly randomized for the mepolizumab ( $n=10$ ) or placebo group ( $n=10)$. In both groups only blood (red arrows) or blood and sputum (green arrows) samples were taken on different days, starting from day 4 after label intake. $1^{\text {st }}$ phase ended at day 60 after label intake. Mepolizumab or placebo was administered for the $1^{\text {st }}$ time at day 4 after label intake. This was followed by a $2^{\text {nd }}$ and $3^{\text {rd }}$ administration of mepolizumab or placebo, each time 28 days later (at day 32 or 60 respectively). B. $2^{\text {nd }}$ phase started at 84 days after the $1^{\text {st }}$ label intake. At this day all patients were again labelled with deuterium glucose and blood was withdrawn. 4 days after the $2^{\text {nd }}$ label intake, the $4^{\text {th }}$ and last mepolizumab or placebo dose was administered. Again either blood only (red arrows) or blood and sputum (green arrows) were taken on several following days.

\section{Blood and sputum collection and processing}

At several time points either blood only (sodium heparin tubes) or a combination of blood and sputum was obtained, see Figure 1. Erythrocytes in blood were lysed in $150 \mathrm{mM} \mathrm{NH}_{4} \mathrm{Cl}, 10 \mathrm{mM} \mathrm{KHCO}_{3}$ and $0.1 \mathrm{mM} \mathrm{NA}_{2}$ EDTA-buffer as described before ${ }^{10}$. Sputum induction was performed as described before ${ }^{6}$. In short, induced sputum samples, obtained after $3-5 \%$ saline inhalation, were processed in $0.1 \%$ sputolysin 
and filtered through a $48 \mu \mathrm{M}$ filter. Blood and sputum leukocytes were washed and resuspended in a staining buffer (PBS2+) consisting of PBS supplemented with $0.32 \%$ $\mathrm{w} / \mathrm{v}$ trisodium citrate and $10 \% \mathrm{w} / \mathrm{v}$ human plasma albumin solution.

\section{Neutrophil nuclear morphology}

Nuclear morphology was determined by manual counting of May-Grünwald-Giemsastained cytospins in a blinded manner. A strict definition was used to identify a separation between lobes in such a way that these were only scored when the connection between lobes was $33 \%$ of the width of the adjacent lobes ${ }^{11}$.

\section{Isolation of neutrophil DNA}

In PBS2+ suspended sputum and blood samples were stained with the following antibodies: CD16-PE/Cy7 (clone 3g8), CD34-Alexa Fluor 700 (clone 581), IL-3R $\alpha$ Brilliant Violet 605 (clone 6H6), CD14 APC-H7 (clone MPHIP9), IL-5R $\alpha-P E$ (clone 26815) and CD9-Cy2 (clone S32). In addition, blood samples were also stained with CD193-Alexa Fluor 647 (clone 5E8) and CD62L-PerCP/Cy5.5 (clone DREG-56) while sputum samples were additionally stained with CD206-PerCP/Cy5.5 (clone 15-2) and CD45-Krome Orange (clone J33).

Thereafter, three neutrophil populations in blood and one neutrophil populations in sputum were collected using an Ariall or ARIAIII FACS sorter. In blood two segmented neutrophil populations were identified based on doublet exclusion, FSC/SSC properties, CD14, ${ }^{-}$CD193- CD16 ${ }^{\text {high }}$ and either CD62 $\mathrm{L}^{\text {high }}$ or CD62 $\mathrm{L}^{\text {dim }}$ (Supplementary Figure 1) ${ }^{12}$. Additionally, SSChigh, CD14 CD16- CD193 and CD62 L $^{-}$neutrophils progenitors were sorted. Sputum neutrophils were gated similarly, with addition of a Live/DEAD stain and CD45 stain to exclude necrotic cells and non-leukocytes (Supplementary Figure 2). Cellular DNA of highly pure sorted neutrophil populations $(>99 \%)$ was isolated from fresh samples using a NucleoSpin blood kit according to producers' instructions.

\section{Determination of DNA ${ }^{2} \mathrm{H}$ enrichment and data analysis}

Collected DNA was enzymatically hydrolysed into deoxy ribonucleotides and derivatized to pentafluoro triacetate derivates (PFTA) as described previously ${ }^{13}$. Thereafter the quantity of ${ }^{2} \mathrm{H}$-labelled and unlabelled adenosine derivatives was measured on an Agilent 7980A/5975C GC-MS in negative chemical ionization mode scanning for $\mathrm{m} / \mathrm{z} 435(\mathrm{M}+0$, unlabelled) and $\mathrm{m} / \mathrm{z} 437(\mathrm{M}+2$, labelled). The resulting enrichments were corrected for natural background enrichment, availability of $6,6-{ }^{2} \mathrm{H}_{2}$-glucose in plasma and the ratio adenosine that is de novo synthesized/ obtained by base salvage $(0.65)$ as published previously ${ }^{9}$. FlowJo v10 was used to analyze the flow cytometry data. Statistical analysis was performed using GraphPad Prism 8.0. 


\section{Results and Discussion}

\section{Presence of three different neutrophil populations in patients with eosinophilic asthma}

We have distinguished three different blood neutrophil populations in our gating strategy based on CD16 and CD62L expression (Supplementary Figure 1) ${ }^{11}$. The "normal" segmented neutrophil population was recognized as CD16 ${ }^{\text {high }}$ and CD62 $\mathrm{L}^{\text {high }}$. CD16 ${ }^{\text {high }}$ and CD62 $L^{\text {dim }}$ cells were also found in the blood of eosinophilic asthma patients. Finally, we also gated for $\mathrm{CD} 16^{\text {neg }}$ and $\mathrm{CD} 62 \mathrm{~L}^{\text {neg }}$ cells which were suspected to be neutrophil progenitors (Supplementary Figure 1$)^{14}$. These three populations were FACS sorted and cytospsins were stained with May-Grünwald-Giemsa and assessed morphologically (Supplementary Figure 1G-I). Indeed, CD16 ${ }^{\text {neg }}$ and CD62 ${ }^{\text {neg }}$ neutrophils had an immature morphology (round nucleus), while CD16 $6^{\text {high }}$ neutrophils had a segmented nucleus. When both CD16 ${ }^{\text {high }}$ neutrophil populations were directly compared on the basis of nuclear morphology, it could be shown that CD16 ${ }^{\text {high }}$ / CD62 $\mathrm{L}^{\text {dim }}$ neutrophils had more segmentation compared to $\mathrm{CD} 16^{\text {high }} / \mathrm{CD} 62 \mathrm{~L}^{\text {high }}$ neutrophils (Figure $2 \mathrm{C}$ ) and were considered to be "hypersegmented neutrophils" as was published before ${ }^{15}$.In sputum, neutrophils were gated as CD16 ${ }^{\text {pos }}$ and CD9 ${ }^{\text {neg }}$ and cytospins of these cells were also assessed morphologically (Supplementary Figure 2). Despite that sputum neutrophils have lower CD16 expression, compared to blood neutrophils, morphologically they had no immature morphology (Supplementary Figure 2F).

\section{Neutrophil numbers are not affected by treatment with mepolizumab}

Determination of the total blood neutrophil count prior to and after 42 days, 56 days and 80 days of treatment with mepolizumab or placebo (Figure $2 \mathrm{~A}$ ), revealed no significant differences (Figure $2 \mathrm{~A}$ ) between the two groups. Also, the percentage of $\mathrm{CD} 16^{\text {high }}$ and $\mathrm{CD} 62 \mathrm{~L}^{\mathrm{dim}}$ neutrophils and the percentage of neutrophil progenitors (Figure $2 \mathrm{~B}$ and $2 \mathrm{D}$ respectively) did not change significantly during and after treatment with mepolizumab. Finally, also the percentage of neutrophils of total leukocytes (CD45 pos cells) in the sputum did not change after treatment with mepolizumab (Figure 2E). This suggests that neutrophil turnover and survival are not likely affected by antiIL-5 treatment but to be certain we also determined ${ }^{2} \mathrm{H}$-label in all above mentioned neutrophil populations. 
A
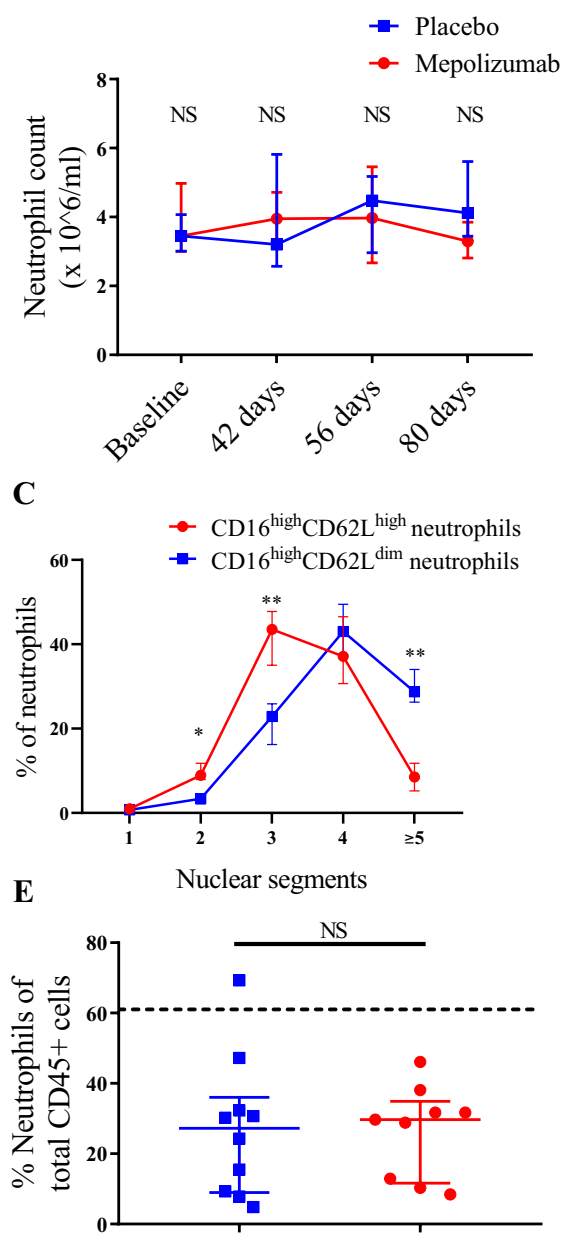

B

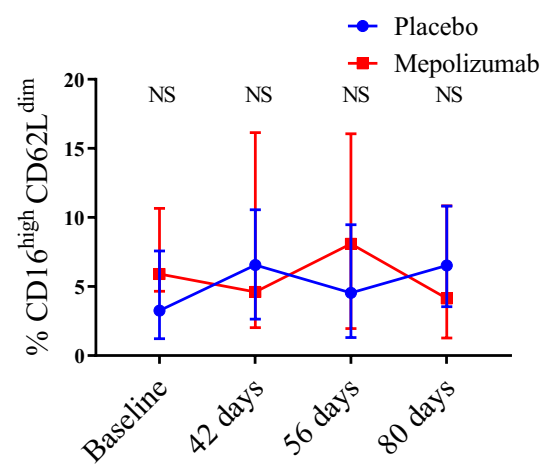

D

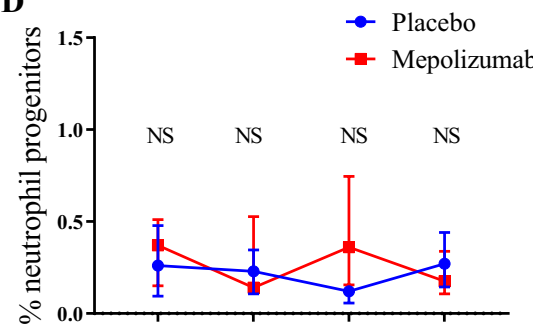

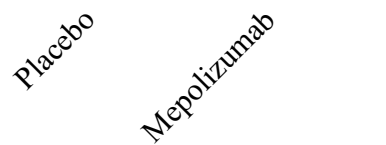

Figure 2. Neutrophil numbers in blood and sputum.

A. The absolute number of blood neutrophils was determined before and several days after administration of mepolizumab (red) or placebo (blue). Similarly, percentage of CD16 $6^{\text {pos }}$ and CD62 Lim neutrophils (B) and neutrophil progenitors (C) was determined before and several days after administration of mepolizumab (red) or placebo (blue). D. From 10 patients in the placebo group and 9 patients in the mepolizumab group the percentage of neutrophils of total leukocytes in the sputum was determined after at least 42 days after mepolizumab (red) or placebo (blue). The dotted line is set at $61 \%$ (cut-off for neutrophilic/ mixed type asthma). Median with interquartile range is indicated in each graph. A Mann Whitney test was performed to compare placebo with mepolizumab at each time point and a Wilcoxon test was performed to compare baseline with the other time points within the same group. NS not significant. 


\section{Neutrophil kinetics in blood and sputum are not altered after treatment with mepolizumab}

Next, we compared the ${ }^{2} \mathrm{H}$-enrichment of blood and sputum neutrophil DNA in 10 patients treated with placebo and 10 patients treated with mepolizumab. Within each group, multiple samples were taken on different days in order to have as much time points as possible. Furthermore, patients of both groups were labelled twice (4 days prior to and 80 days after the first administration of mepolizumab or placebo) in order to have information about short term and long-term effect of anti-IL-5 (Figure 1). For the sputum samples the time points were strategically chosen close to and far from both labelling days, because we hypothesized that precisely these days would show putative differences in label enrichment.

During both labelling phases, progression of ${ }^{2} \mathrm{H}$-label in the DNA of $\mathrm{CD} 16^{\text {high }} \mathrm{CD} 62 \mathrm{~L}^{\text {high }}$ (normal) neutrophils was largely similar between the mepolizumab and the placebo group (Figure $3 \mathrm{~A}$ and $3 \mathrm{~B}$ ). Also, when ${ }^{2} \mathrm{H}$-enrichement in multiple time points from day 8 and beyond was compared between the two groups, there were no statistically significant differences. So, the slight differences seen between the two groups were most likely caused by variance within donors rather than differences between the altered treatment. We came to a similar conclusion when we compared ${ }^{2} \mathrm{H}$ enrichment in the DNA of $C D 16^{\text {high }} \mathrm{CD} 62 \mathrm{~L}^{\mathrm{dim}}$ (Figure $3 \mathrm{C}$ and $3 \mathrm{D}$ ) and $\mathrm{CD} 16^{\text {neg }} \mathrm{CD} 62 \mathrm{~L}^{\text {neg }}$ (progenitors, Figure $3 \mathrm{E}$ and $3 \mathrm{~F}$ ) neutrophils. Lastly, also ${ }^{2} \mathrm{H}$-enrichement of sputum neutrophils was largely similar between the two groups (Figure $3 \mathrm{G}$ and $3 \mathrm{H}$ ).

Interestingly, a decrease in eosinophil inflammation seen after mepolizumab ${ }^{16}$, did not lead to a difference in the number or kinetics of neutrophils in blood and sputum.

\section{Neutrophil progenitors and hypersegmented neutrophils have different kinetics compared to "normal" neutrophils}

Since, neutrophil kinetics was not altered by mepolizumab in both phases of this study, we decided to pool all time points from both treatment groups and phases of the study in order to investigate possible differences in kinetics between the different neutrophil populations in the blood with as many data points as possible. Doing so, we discovered that the two blood neutrophil populations $C D 16^{\text {high }} C D 62 L^{\text {dim }}$ and $C D 16^{\text {neg }} C D 62 L^{\text {neg }}$ (neutrophil progenitors) both exhibit different kinetics compared to the "normal" $\mathrm{CD} 16^{\text {high }} \mathrm{CD} 62 \mathrm{~L}^{\text {high }}$ neutrophils (Figure 4A). The neutrophil progenitors were indeed earlier ${ }^{2} \mathrm{H}$-labelled, with the highest enrichment during the earliest time point ( 3 after label intake). It is, however, very likely that the peak enrichment for the progenitors was missed, because the earlier time points (day 1 and 2 after label intake) were not sampled. In mice the peak enrichment for preNeus (also neutrophil progenitors) in the bone marrow was reached 24 hours after BrdU pulse intake ${ }^{17}$. We have shown similar kinetics for neutrophil progenitors (neutrophilic promyelocytes and myelocytes) in chapter 2 , however the peak was extended to 48 hours after label intake in humans. Therefore, it can be argued that neutrophil progenitors display similar kinetics independent of location (blood versus bone marrow aspiration).

CD16 ${ }^{\text {high }}$ CD62 $L^{\text {dim }}$ neutrophils in asthma patients have a morphology with a hypersegmented nucleus, similar as was seen in healthy volunteers that underwent endotoxemia challenge ${ }^{18}$. These hypersegmented neutrophils seen after endotoxemia, which is a model for acute inflammation, did not seem to exhibit a difference in kinetics 
compared to normal segmented neutrophils but the different time points were two days apart. In this study analyzing time points 1 day apart, the hypersegmented neutrophils displayed a small delay of approximately one day compared to normal segmented neutrophils (CD16 ${ }^{\text {high }} \mathrm{CD} 62 \mathrm{~L}^{\text {high }}$ neutrophils, Figure $4 \mathrm{~A}$ ). This finding fits with the hypothesis that hypersegmented neutrophils in asthma are more matured than normal segmented neutrophils. This discovery is in line with earlier published data on CD62 $\mathrm{L}^{\mathrm{dim}}$ and CXCR4 ${ }^{\text {high }}$ neutrophils which were considered to be aged neutrophils, ready to home back to the bone marrow in order to be eliminated ${ }^{19}$. However, the alternative hypothesis of hypersegmented neutrophils as separate subset ${ }^{15}$ cannot be ruled out. This second hypothesis is supported by the clear difference in proteome between mature and hypersegmented cells ${ }^{18}$.

A

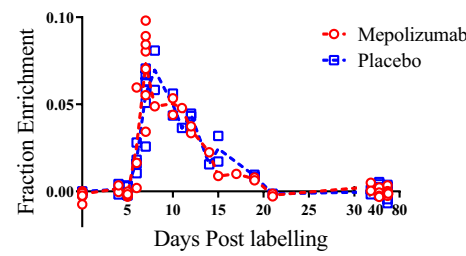

C CD62L ${ }^{\text {dim }}$ neutrophils blood 1st phase

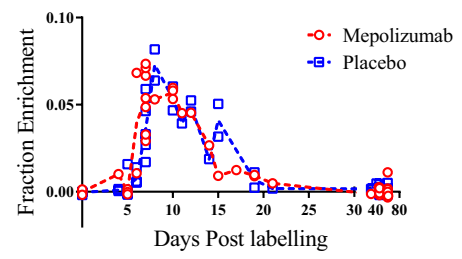

$\mathbf{E}$

Neutrophil progenitors blood 1st phase

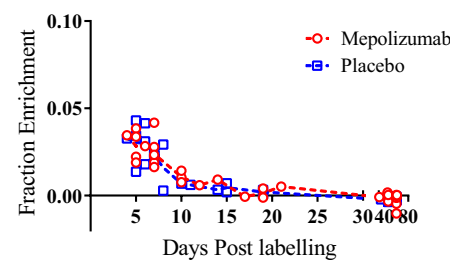

G

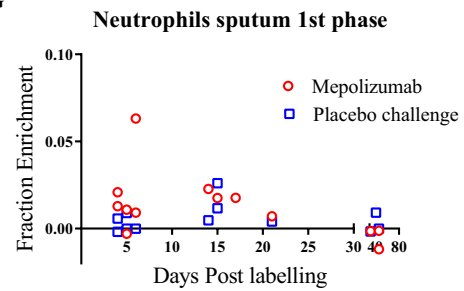

B CD62L $\mathrm{L}^{\text {high }}$ neutrophils blood 2nd phase

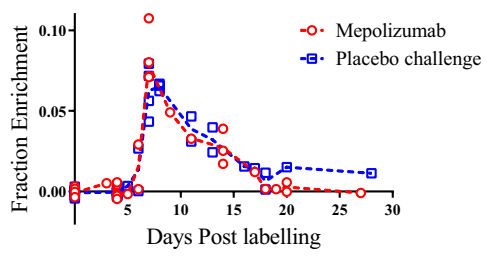

D CD62 $L^{\text {dim }}$ neutrophils blood 2nd phase

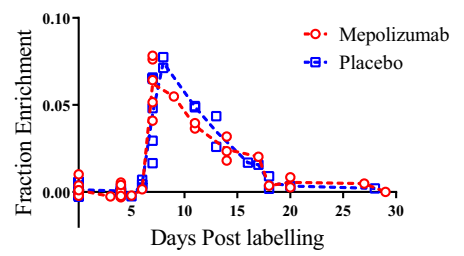

$\mathbf{F}$ Neutrophil progenitors blood 2nd phase

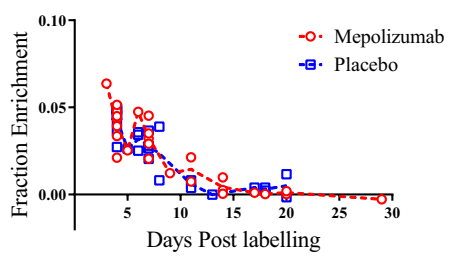

H

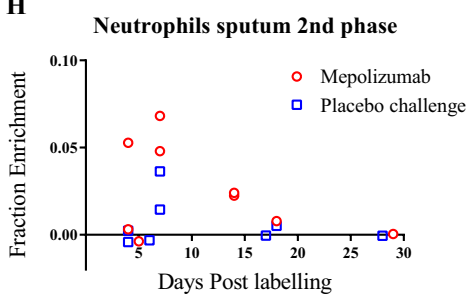

Figure 3. Deuterium enrichment of blood and sputum neutrophil DNA.

A. The ratio of ${ }^{2} \mathrm{H}$-labelled and total DNA of normal blood neutrophils (CD16 ${ }^{\text {high }}$ and $\mathrm{CD} \mathrm{L}^{\mathrm{L}}{ }^{\text {high }}$ ) was determined before label intake and at several days after label intake for patients in the placebo (blue) and the mepolizumab group (red). This ratio was corrected for each individual's plasma glucose enrichment 
and the factor 0.65 (only $65 \%$ of the adenosine moieties can be enriched with deuterium when there is $100 \%$ deuterium availability). B. This was similarly done for "normal" blood neutrophils in both groups after the second label intake. This was equally done for $\mathrm{CD} 16^{\text {high }} \mathrm{CD} 62 \mathrm{~L}^{\mathrm{dim}}$ neutrophils during both phases ( $C$ and $\mathbf{D}$ ) and for neutrophil progenitors ( $\mathbf{E}$ and $\mathbf{F}$ ) and finally also for sputum neutrophils ( $\mathbf{G}$ and $\mathbf{H}$ ). In A-F the individual data points are plotted together with median with interquartile range. A dotted line connects the median values of each day with the other day. G-H only the individual data points are shown.

\section{Sputum neutrophils follow the same kinetics as blood neutrophils}

In almost all patients sufficient neutrophils in the sputum were sampled at three different time points in order to quantify ${ }^{2} \mathrm{H}$-enrichement in the DNA. All sputum samples were pooled together in one graph, because, there were no evident differences between the two treatment groups or the two phases of the study (Figure 3G-H), (Figure 4B). These sputum samples were compared to the paired blood samples of the $\mathrm{CD} 16^{\text {high }} \mathrm{CD} 62 \mathrm{~L}^{\text {high }}$ and the $\mathrm{CD} 16^{\text {high }} \mathrm{CD} 62 \mathrm{~L}^{\text {dim }}$ neutrophils (Figure 4B). We also included blood and sputum data points from an earlier study in which similar (non-randomized for treatment with mepolizumab or placebo) eosinophilic asthma patients (EHLA study) were enrolled in order to have more information about ${ }^{2} \mathrm{H}$-enrichment between day 8 and 13 after label intake. This revealed that at all time points the sputum samples showed almost identical ${ }^{2} \mathrm{H}$-enrichment as the blood neutrophils. This finding implies that sputum cells are not long-lived in the sputum or bronchial airways and are most likely continuously replaced by neutrophils from the circulation. Alternatively, a continuous exchange of neutrophils from blood and sputum cannot be ruled out. Whatever, the hypothesis the similar label kinetics of neutrophils in the two compartments implies that neutrophil circulatory time is similar to the neutrophil time of survival in the tissues. This outcome is in contrast to what was displayed in mice, in which neutrophils are believed to stay as long as 6 days in the lungs before they are cleared ${ }^{20}$. 
A

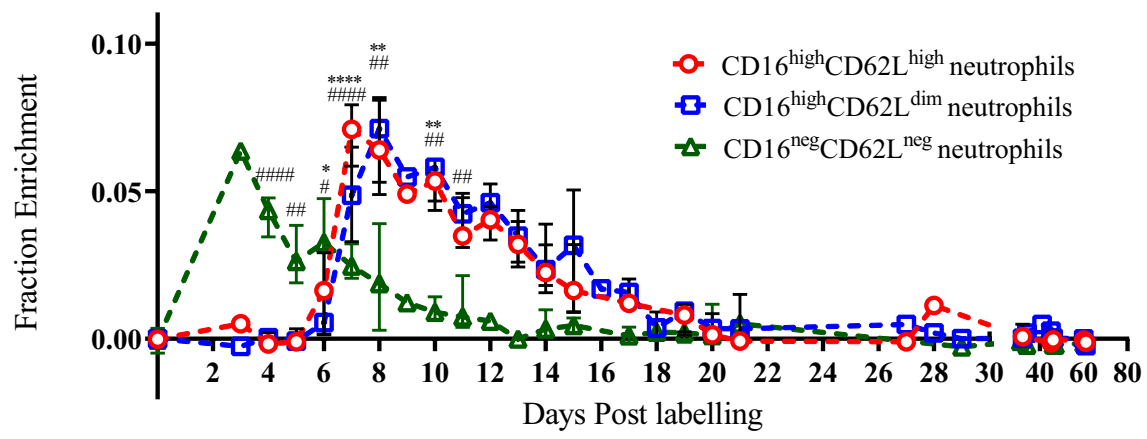

B

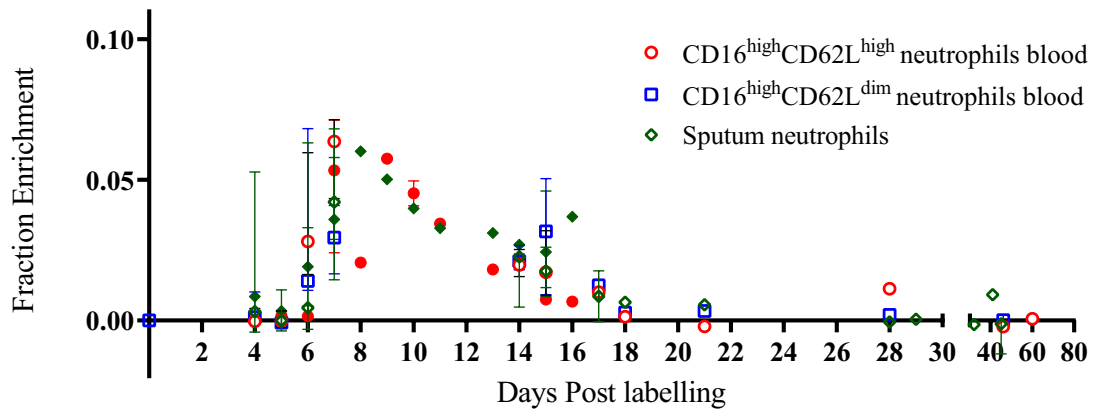

Figure 4. Deuterium enrichment of the pooled data from paired blood and sputum neutrophils.

A. All data points of ${ }^{2} \mathrm{H}$-enrichment from the three neutrophil populations in the blood (from Figure 3) were pooled and plotted in this graph. Green open triangles represent the neutrophil progenitors $\left(C D 16^{\text {neg }} C D 62 L^{\text {neg }}\right)$, red open circles represent the normal segmented neutrophils (CD16 high $C D 62 L^{\text {high }}$ ) and the blue open squares represent the hypersegmented neutrophils. A repeated measures 2-way ANOVA with Turkey's correction for multiplicity was performed to compare differences in enrichment between the three neutrophil populations within each time point. \# indicates $p \leq 0.05$ for $\mathrm{CD} 16^{\text {high }} \mathrm{CD} 62 \mathrm{~L}^{\text {high }}$ versus $\mathrm{CD} 16^{\text {neg }} \mathrm{CD} 62 \mathrm{~L}^{\text {neg }}$, * indicates $\mathrm{p} \leq 0.05$ for $\mathrm{CD} 16^{\text {high }} \mathrm{CD} 62 \mathrm{~L}^{\text {high }}$ versus $\mathrm{CD} 16^{\text {high }} \mathrm{CD} 62 \mathrm{~L}^{\text {dim }}$; \#\# indicates $p \leq 0.01$ for $C D 16^{\text {high }} C D 62 L^{\text {high }}$ versus $C D 16^{\text {neg }} C D 62 L^{\text {neg }}, * *$ indicates $p \leq 0.01$ for $C D 16^{\text {high }} C D 62 L^{\text {high }}$ versus $C D 16^{\text {high }} C D 62 L^{\text {dim }}$, \#\#\#\# indicates $p \leq 0.001$ for $C D 16^{\text {high }} C D 62 L^{\text {high }}$ versus $C D 16^{\text {neg }} C D 62 L^{\text {neg, }}, * * * *$ indicates $p \leq 0.001$ for $\mathrm{CD} 16^{\text {high }} \mathrm{CD} 62 \mathrm{~L}^{\text {high }}$ versus $\mathrm{CD} 16^{\text {high }} \mathrm{CD} 62 \mathrm{~L}^{\text {dim }}$. B. This was similarly done for sputum neutrophils (open red circles indicate segmented neutrophils from blood, blue open squares indicate the hypersegmented neutrophils, while the open green diamonds indicate the paired neutrophils from the sputum samples). Additional data points from an earlier asthma study were added ( $n=9)$ in order to have more data points per day. These data points are indicated with closed symbols (closed red circles for segmented blood neutrophils and closed green diamonds for sputum neutrophils). Medians with interquartile range are indicated. All data points were statistically tested, but only statistically significant results are indicated. 


\section{In conclusion}

Taken together, the absolute number and kinetics of blood and sputum neutrophils in patients with eosinophilic asthma are not altered by treatment with Mepolizumab (anti-IL-5). This implies to tissue damage present in eosinophil asthma does not lead to an appreciable change in neutrophil kinetics. On the other hand, three different blood neutrophil populations with distinct kinetics were found in the circulation of these patients. $\mathrm{CD} 16^{\mathrm{neg}} \mathrm{CD} 62 \mathrm{~L}^{\text {neg }}$ neutrophils have an immature nuclear morphology and incorporate label as early as three days after label intake. Therefore, it is very likely that these cells are circulatory neutrophil progenitors (neutrophil promyelocytes and myelocytes). $\mathrm{CD} 16^{\text {high }} \mathrm{CD} 62 \mathrm{~L}^{\text {high }}$ are most likely normal segmented neutrophils and $\mathrm{CD} 16^{\text {high }} \mathrm{CD} 62 \mathrm{~L}^{\text {dim }}$ neutrophils are hypersegmented neutrophils. These hypersegmented neutrophils have one day delay in label enrichment compared to the normal segmented neutrophils, which makes them most likely more mature. Finally, blood and sputum neutrophils follow the same enrichment, indicating that neutrophils have a short residence time in the sputum or are in complete exchange between both compartments.

\section{Acknowledgements}

The authors thank Jeroen van Velzen and Pien van der Burght for help with the flow cytometry sorting. 


\section{References}

1. Brooks CR, Van Dalen CJ, Harding E, Hermans IF, Douwes J. Effects of treatment changes on asthma phenotype prevalence and airway neutrophil function. BMC Pulm Med [Internet]. 2017 [cited 2020 Jan 27];17:169. Available from: http://www.ncbi.nlm.nih.gov/ pubmed/29202821

2. Simpson JL, McElduff P, Gibson PG. Assessment and reproducibility of non-eosinophilic asthma using induced sputum. Respiration [Internet]. 2010 [cited 2020 Jan 27];79:147-51. Available from: http://www.ncbi.nlm.nih.gov/pubmed/19816004

3. Fowler SJ, Tavernier G, Niven R. High blood eosinophil counts predict sputum eosinophilia in patients with severe asthma. J Allergy Clin Immunol [Internet]. 2015 [cited 2020 Jan 27];135:822-4.e2. Available from: http://www.ncbi.nlm.nih.gov/pubmed/25445828

4. Yancey SW, Keene ON, Albers FC, Ortega H, Bates S, Bleecker ER, et al. Biomarkers for severe eosinophilic asthma. J Allergy Clin Immunol [Internet]. 2017 [cited 2020 Jan 27];140:150918. Available from: http://www.ncbi.nlm.nih.gov/pubmed/29221581

5. Simpson JL, Scott R, Boyle MJ, Gibson PG. Inflammatory subtypes in asthma: assessment and identification using induced sputum. Respirology [Internet]. 2006 [cited 2019 Dec 5];11:54-61. Available from: http://www.ncbi.nlm.nih.gov/pubmed/16423202

6. Tak T, Hilvering B, Tesselaar K, Koenderman L. Similar activation state of neutrophils in sputum of asthma patients irrespective of sputum eosinophilia. Clin Exp Immunol [Internet]. 2015 [cited 2020 Jan 27];182:204-12. Available from: http://www.ncbi.nlm.nih. gov/pubmed/26148992

7. Denlinger LC, Sorkness RL, Lee WM, Evans MD, Wolff MJ, Mathur SK, et al. Lower airway rhinovirus burden and the seasonal risk of asthma exacerbation. Am J Respir Crit Care Med [Internet]. 2011;184:1007-14. Available from: http://www.ncbi.nlm.nih.gov/ pubmed/21816938

8. Pittman K, Kubes P. Damage-associated molecular patterns control neutrophil recruitment. J Innate Immun [Internet]. 2013 [cited 2020 Jan 30];5:315-23. Available from: http://www. ncbi.nlm.nih.gov/pubmed/23486162

9. Macallan DC, Asquith B, Zhang Y, de Lara C, Ghattas H, Defoiche J, et al. Measurement of proliferation and disappearance of rapid turnover cell populations in human studies using deuterium-labeled glucose. Nat Protoc [Internet]. 2009 [cited 2019 Sep 3];4:1313-27. Available from: http://www.nature.com/articles/nprot.2009.117

10. Chernyshev A V, Tarasov PA, Semianov KA, Nekrasov VM, Hoekstra AG, Maltsev VP. Erythrocyte lysis in isotonic solution of ammonium chloride: theoretical modeling and experimental verification. J Theor Biol [Internet]. 2008 [cited 2019 Dec 12];251:93-107. Available from: http://www.ncbi.nlm.nih.gov/pubmed/18083194

11. van Grinsven E, Textor J, Hustin LSP, Wolf K, Koenderman L, Vrisekoop N. Immature Neutrophils Released in Acute Inflammation Exhibit Efficient Migration despite Incomplete Segmentation of the Nucleus. J Immunol [Internet]. 2019 [cited 2020 Mar 11];202:207-17. Available from: http://www.ncbi.nlm.nih.gov/pubmed/30504419

12. Pillay J, Kamp VM, van Hoffen E, Visser T, Tak T, Lammers J-W, et al. A subset of neutrophils in human systemic inflammation inhibits $T$ cell responses through Mac-1. J Clin Invest [Internet]. 2012 [cited 2019 Aug 5];122:327-36. Available from: http://www.jci.org/ articles/view/57990 
13. Busch R, Neese RA, Awada M, Hayes GM, Hellerstein MK. Measurement of cell proliferation by heavy water labeling. Nat Protoc [Internet]. 2007 [cited 2019 Sep 3];2:3045-57. Available from: http://www.ncbi.nlm.nih.gov/pubmed/18079703

14. Cortjens B, Ingelse SA, Calis JC, Vlaar AP, Koenderman L, Bem RA, et al. Neutrophil subset responses in infants with severe viral respiratory infection. Clin Immunol [Internet]. 2017 [cited 2020 Jan 28];176:100-6. Available from: http://www.ncbi.nlm.nih.gov/ pubmed/28108365

15. Pillay J, Tak T, Kamp VM, Koenderman L. Immune suppression by neutrophils and granulocytic myeloid-derived suppressor cells: similarities and differences. Cell Mol Life Sci [Internet]. 2013 [cited 2018 Oct 4];70:3813-27. Available from: http://www.ncbi.nlm.nih. gov/pubmed/23423530

16. Hassani M, Koenderman L. Immunological and hematological effects of IL-5(R $\alpha)$-targeted therapy: An overview. Allergy [Internet]. 2018 [cited 2019 Nov 7];73:1979-88. Available from: http://www.ncbi.nlm.nih.gov/pubmed/29611207

17. Evrard M, Kwok IWH, Chong SZ, Teng KWW, Becht E, Chen J, et al. Developmental Analysis of Bone Marrow Neutrophils Reveals Populations Specialized in Expansion, Trafficking, and Effector Functions. Immunity [Internet]. 2018 [cited 2018 Jul 24];48:364-379.e8. Available from: http://www.ncbi.nlm.nih.gov/pubmed/29466759

18. Tak T, Wijten P, Heeres M, Pickkers P, Scholten A, Heck AJR, et al. Human CD62 Ldim neutrophils identified as a separate subset by proteome profiling and in vivo pulse-chase labeling. Blood [Internet]. 2017 [cited 2020 Jan 14];129:3476-85. Available from: http:// www.ncbi.nlm.nih.gov/pubmed/28515092

19. Casanova-Acebes M, Pitaval C, Weiss LA, Nombela-Arrieta C, Chèvre R, A-González N, et al. Rhythmic modulation of the hematopoietic niche through neutrophil clearance. Cell [Internet]. 2013 [cited 2020 Jan 31];153:1025-35. Available from: http://www.ncbi.nlm. nih.gov/pubmed/23706740

20. Cheretakis C, Leung R, Sun CX, Dror Y, Glogauer M. Timing of neutrophil tissue repopulation predicts restoration of innate immune protection in a murine bone marrow transplantation model. Blood [Internet]. 2006 [cited 2020 Jan 31];108:2821-6. Available from: http://www. ncbi.nlm.nih.gov/pubmed/16804110 
A

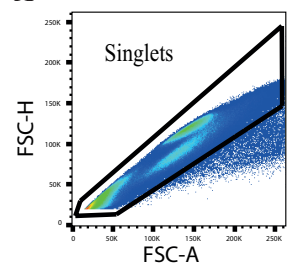

$\mathbf{E}$

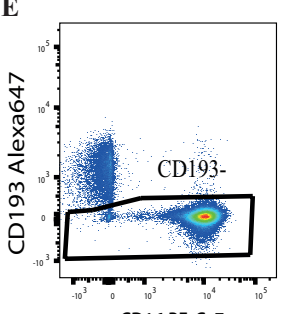

CD16 PE-Cy7
B

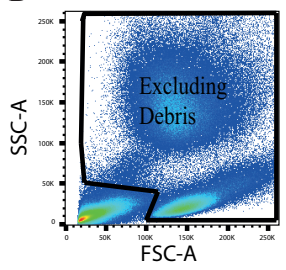

F

$\mathbf{F}$

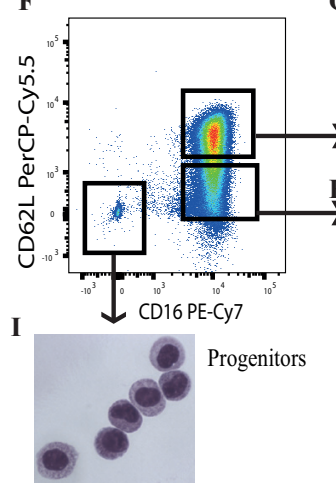

$\mathrm{C}$

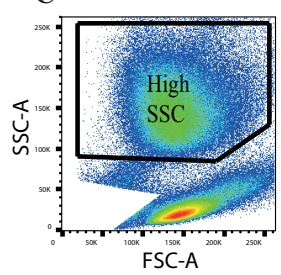

G
D

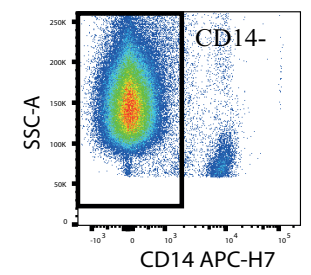

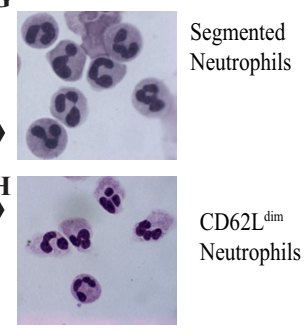

Supplementary Figure 1. Gating strategy for FACS sorting neutrophils in the blood.

Three distinct neutrophil populations were identified by gating on singlets on the basis of FSC-A and FSC- $\mathrm{H}$ (A), debris is excluded on the basis of FSC-A and SSC-A (B), subsequently only high SSC-A cells are gated (C), CD14 high cells are excluded (D), CD193 ${ }^{\text {pos }}$ cells were excluded (E) and finally within CD16 and CD62L plot three neutrophil populations were gated (F). Segmented neutrophils were gated as $C D 16^{\text {high }} \mathrm{CD} 62 \mathrm{~L}^{\text {high }}$ $(\mathrm{G})$, hypersegmented neutrophils were gated as $\mathrm{CD} 16^{\text {high }} \mathrm{CD} 62 \mathrm{~L}^{\text {dim }}$ neutrophils $(\mathrm{H})$ and finally neutrophil progenitors were gated as $\mathrm{CD} 16^{\text {neg }} \mathrm{CD} 62 \mathrm{~L}^{\text {neg. }}$. These cells were FACS sorted and cytospins were stained with May-Grünwald Giesma (MMG) and microscopically evaluated. Three examples of representative microscopic images (objective 100x) of cytospins of sorted neutrophils are shown (G-I). 
A

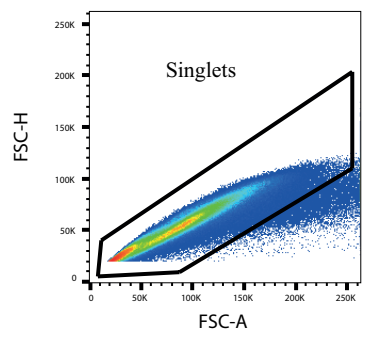

D

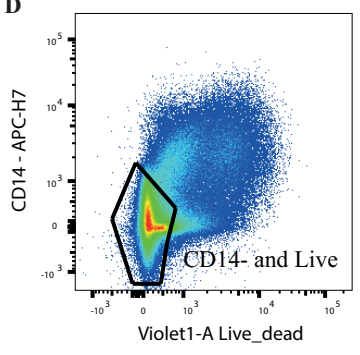

B

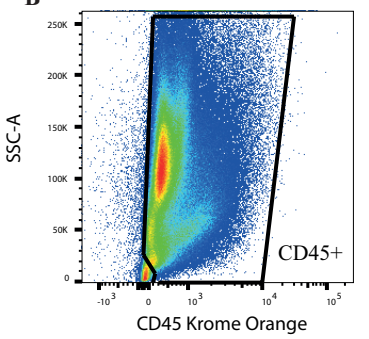

$\mathbf{E}$

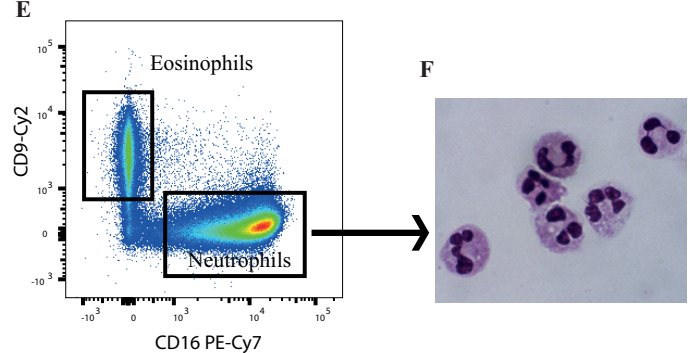

Supplementary Figure 2. Gating strategy for FACS sorting neutrophils in the sputum.

Sputum neutrophils were gated after gating singlets (A), CD45pos cells (B), SSC high cells $(\mathbf{C})$ and CD14 ${ }^{\text {neg }}$ and negative for live/dead stain (D). Finally, neutrophils were gated as CD16 ${ }^{\text {pos }}$ and CD $9^{\text {neg }}$ cells and FACS sorted (E). A representative microscopic image (100x objective) of an MMG stained cytospin of sorted neutrophils is shown (F). 


\section{Unique cross-talk between GM-CSF and CXCR4 signaling in human neutrophils.}

Marwan Hassani ${ }^{1}$, Daan van Spengler ${ }^{1}$, Lucas Picavet ${ }^{2}$, Naomi van Leeuwenstijn ${ }^{1}$, Deon Kanters ${ }^{1}$, Nienke Vrisekoop ${ }^{1}$, Leo Koenderman ${ }^{1}$

1. Department of Respiratory Medicine and Center for Translational Immunology, University Medical Center Utrecht, The Netherlands.

2. Center for Translational Immunology, University Medical Centre Utrecht, Utrecht, The Netherlands. 


\section{Abstract}

The CXCL12/CXCR4 signaling axis is considered to be important in the regulation of neutrophil trafficking from and to the bone marrow. The consensus in the literature is that only in vitro aged neutrophils have significant expression of CXCR4 on the surface membrane and hence can respond to CXCL12.

Here we have investigated the role of CXCR4 in freshly isolated neutrophils. Moreover, we studied the putative cross-talk between cytokine receptors and CXCR4 signaling in human neutrophils.

Freshly isolated neutrophils respond to CXCL12 with a marked increase in the cytosolic free $\mathrm{Ca}^{2+}$ concentration $\left(\left[\mathrm{Ca}^{2+}\right]_{\mathrm{i}}\right)$, despite a very low expression of CXCR4 on the membrane. This change in $\left[\mathrm{Ca}^{2+}\right]_{i}$ was uniquely and rapidly (15-30 minutes) blocked by the GM-CSF receptor signaling through its common ß-chain. This inhibition is initiated via the activation of JAK2 and is not associated with a decrease of CXCR4 expression on the membrane. A putative role for the actin cytoskeleton in the inhibition of CXCR4 was demonstrated by marked changes upon addition of modulators of the actincytoskeleton.

Our study demonstrates that cross-talk between the GM-CSF and the CXCR4 receptor on human neutrophils drastically changes CXCL12 induced signaling. This finding might be clinically relevant, as it might underlie the mechanism by which treatment with GM-CSF induce acute egress of neutrophils from the bone marrow to the blood. 


\section{Introduction}

Fifty chemokines and twenty chemokine receptors are known in the human immune system. These receptors are sub-categorized into four groups: C, CC, CX3C and CXC, based on the pattern of cysteine residues of their ligands ${ }^{1}$. CXCR4 is part of the CXC receptor family and specifically binds CXCL12, previously known as stromal derived factor-1 $\alpha$ (SDF-1 $\alpha)^{2}$. CXCR4 is expressed on the surface of most peripheral white blood cells, but large amounts of receptor protein are stored intracellularly ${ }^{3}$. The initial identification of this receptor was, together with CCR5, related to its role in entry of HIV into $\mathrm{CD} 4^{+}$cells ${ }^{4}$. Furthermore, expression of CXCR4 on various cancer cells is considered to play an important role in cancer metastasis ${ }^{5}$.

In neutrophils the CXCL12/CXCR4 signaling axis is considered to be involved in the egress and homing of neutrophils and their progenitors from and to the bone marrow ${ }^{6}$. The importance of CXCR4 in the regulation of neutrophil egress from the bone marrow is clearly illustrated in patients suffering from WHIM syndrome ${ }^{7}$. These patients have a mutated hyperactive (gain-of-function) CXCR4 receptor, resulting in a strongly reduced release from the bone marrow of leukocytes, including neutrophils, into the circulation; a mechanism referred to as myelokathexis ${ }^{7}$.

On the other hand, several studies have suggested that CXCR4 expression increases during neutrophil aging and that senescent neutrophils with high CXCR4 expression home back to the bone marrow in order to be cleared ${ }^{8,9,10}$. This mechanism was supported by the finding that freshly isolated peripheral blood neutrophils exhibited a very low if any expression of CXCR4. As a result these cells do not seem to be responsive to CXCL12 in terms of chemotaxis ${ }^{11,12}$. It was also suggested that CXCL12 did not activate signal transduction pathways in freshly isolated neutrophils. These studies showed that $\mathrm{PI3K} / \mathrm{AKT} / \mathrm{mTOR}$ and ERK1/2 pathways in neutrophils were not activated by CXCL12 ${ }^{11,13}$. Similar to all G-protein coupled receptors, CXCR4 activation also leads to the dissociation of the trimeric G-protein liberating the $\alpha$ and by subunits. The $\alpha$-subunit in turn will activate phospholipase $C$ leading to production of inositol-trisphosphate (Ins3P) and 1,2-diacylglycerol. Ins3P triggers the mobilization of $\mathrm{Ca}^{2+}$ from the endoplasmic reticulum into the cytosol ${ }^{14}$. $\mathrm{Ca}^{2+}$ is an important second messenger involved in several neutrophil responses such as the migration machinery, actin polarization and focal adhesion dynamics of a cell ${ }^{15}$. However, actin reorganization per se is also possible in the absence $\mathrm{Ca}^{2+}$ signalling ${ }^{16}$. Besides, several cytokines such as GM-CSF, G-CSF and IL-4 are able to inhibit GPCR signaling, presumably by activating beta-arrestins via activation of GRK which will consequently lead to GPCR internalization ${ }^{17}$.

The aim of this article was to gain better insights into the role of CXCR4 in activation of freshly isolated peripheral blood neutrophils from healthy humans. This article will show that freshly isolated neutrophils show a clear $\left[\mathrm{Ca}^{2+}\right]_{i}$ response after stimulation with CXCL12, whereas this chemokine does not lead to significant chemotaxis in these neutrophils. Moreover, we show that this CXCL12 induced $\mathrm{Ca}^{2+}$ influx is rapidly and specifically inhibited by GM-CSF. 


\section{Materials and methods}

\section{Blood processing and granulocyte isolation}

Blood was obtained from healthy donors at the university medical center Utrecht (UMCU) in sodium heparin tubes (Vacuette ${ }^{\circledR}$ Greiner bio-one, Kremsmünster, Austria). Erythrocytes were lysed using an ice-cold isotonic lysis bufferconsisting of $150 \mathrm{mMNH}_{4} \mathrm{Cl}, 10 \mathrm{mMKHCO}_{3}$ and $0.1 \mathrm{mM} \mathrm{NA}_{2}$ EDTA. Leukocytes were then washed and suspended in a staining buffer, (PBS2+) containing PBS supplemented with $0.32 \% \mathrm{w} / \mathrm{v}$ trisodium citrate (both prepared by the UMCU pharmacy) and $10 \% \mathrm{w} / \mathrm{v}$ human serum albumin (Sanquin, Amsterdam, the Netherlands). Granulocytes were isolated using blood of healthy volunteers which was diluted 1:1 with PBS2+ and overlaid on top of Ficoll-Paque and centrifuged for 20 min at $760 \mathrm{xg}$ at $20{ }^{\circ} \mathrm{C}$. Erythrocytes were similarly lysed as described above. All preparations contained $>95 \%$ granulocytes.

\section{Neutrophil stimulation}

Prior to stimulation, neutrophils were suspended in HEPES buffer (20 mM HEPES, 132 $\mathrm{mM} \mathrm{NaCl}, 6 \mathrm{mM} \mathrm{KCl}, 1 \mathrm{mM} \mathrm{MgSO} 4,1.2 \mathrm{mM} \mathrm{KH} 2 \mathrm{PO}$ ) containing $5 \mathrm{mM}$ glucose,1.0 mM $\mathrm{CaCl}_{2}$ and $0,5 \%$ human serum albumin. Then neutrophils were stimulated with several reagents (Table 1). Stimulation of neutrophils was performed in an incubator, set at $37^{\circ} \mathrm{C}$.

\section{ROS production}

Production of ROS was studied by incubating freshly isolated neutrophils in a 96well plate in the presence of, Amplex Red $(20 \mu \mathrm{M}$, Molecular Probes, Leiden, The Netherlands), and Horseradish peroxidase, HRP (0.25 U/ml, Sigma Chemical, St. Louis, MO, USA). Production of fluorescent resorufin, formed from Amplex Red in the presence of HRP after peroxidation, was measured during 1 hour at $37^{\circ} \mathrm{C}$ using a fluoroluminometer FluostarOptima (BMG labtech, Ortenberg, Germany).

\section{Western Blotting}

Isolated and stimulated neutrophils or PBMCs (7-10 million per sample) were washed and resuspended in $100 \mu$ ice cold PBS containing ( 1 tablet per $10 \mathrm{ml}$ ) protease inhibitor cocktail (Sigma-Aldrich, St. Louis, MO, USA) and 10 mM DFP (Sigma-Aldrich, St. Louis, MO, USA). Thereafter the samples were lysed with $200 \mu \mathrm{L} 4 \mathrm{x}$ Laemmli sample buffer ( $62.5 \mathrm{mM}$ Tris-HCl pH 6.8, 1\% SDS and 10\% glycerol) containing 2 mM DTT (Duchefa Farma, Haarlem, the Netherlands) and 1 mM PMSF (Sigma-Aldrich, St. Louis, MO, USA) and boiled for 8 min at $98^{\circ} \mathrm{C}$. $40 \mu \mathrm{l}$ sample was loaded each time. Proteins were separated by SDS-PAGE (Bio-Rad, Hercules, CA, USA) and transferred to a polyvinylidene difluoride membrane (Merck, Darmstadt, Germany). The blots were blocked in hybridization buffer (10 mM Tris, $150 \mathrm{mM} \mathrm{NaCl}$ and $0.1 \%$ Tween-20) containing 10\% milk powder (ELK, Campina, The Netherlands) for $1 \mathrm{~h}$ followed by incubation with polyclonal anti-CXCR4 (AHP442, BioRad, Hercules, CA, USA) or anti-phospho-ERK1/2 (Cell signaling technology, Danvers, MA, USA) or anti-ERK1/2 (Cell signaling technology, Danvers, MA, USA) in hybridization buffer with $1 \%$ ELK overnight at $4^{\circ} \mathrm{C}$. The secondary Swine anti rabbit-HRP antibody (1:2000) (Santa Clara, CA, USA) was incubated in hybridization buffer containing 1\% ELK for 1 hour at ambient temperature. Detection of all Western blots were performed by enhanced chemiluminescence (Thermo Fisher Scientific, Waltham, MA, USA). 


\section{Flow cytometry and ImageStream}

After lysing erythrocytes as is described above, 1 million leukocytes, in a concentration of 40 million per $\mathrm{ml}$ were resuspended in PBS2+, also containing $5 \%$ normal mouse serum (Bio-Connect Huissen, the Netherlands) and 5\% FcR blocking reagent (Miltenyi Biotec, Bergisch Gladbach, Germany). Cells were stained with anti-CXCR4: PE (12G5, Biolegend, San Diego, CA, USA) or IgG1 isotype PE labeled (11711, R \& D Systems, Minneapolis, MN, USA). Moreover, the following antibodies and stainings were used: CD16-FITC (clone 3g8), CD14-FITC (clone M5E2), CD35-FITC (clone E11), CD62L-BV650 (clone DREG56), CD66b-PerCPCy5.5 (clone G10F5), CD49d-PECy7 (clone 9F10), CD64AF647 (clone 10.1), CD11b-AF700 (clone CBRM1/5), CD11b-AF750 (clone Bear1), CD14-eF450 (clone 61D3), CD16-KO (clone 3g8) ,7-AAD and CD3-FITC (clone UCHT1). Canto II cell analyzer or LSRFortessa (Becton Dickinson, Mountain view CA, USA) was used to quantitatively measure surface expression whereas the Amnis ImageStreamX Mk II Imaging Flow Cytometer (Luminexcorp, Austin, TX, USA) was used to visualize CXCR4 expression on the surface membrane.

\section{Neutrophil chemotaxis assay}

Isolated neutrophils were resuspended inside a fibrin gel (containing $2 \mathrm{mg} / \mathrm{ml}$ fibrinogen (Sigma-Aldrich) with $1 \mathrm{U} / \mathrm{ml}$ thrombin (Sigma-Aldrich)) and placed in the center of a 10 $\mu$-slide chemotaxis chamber (ibidi GmbH, Fitchburg, WI, USA). The chemotactic agent (CXCL12 or fMLF) was added in the chamber above the neutrophils, while Hepes buffer was added below. Cells were visualized under a bright field microscope ( $5 x$ magnification) and movement was tracked using special software as was published before ${ }^{18}$.

\section{Statistical analyses}

FlowJo v10 (LLC, Ashland, OR, USA) was used to analyze the flow cytometry data. Statistical analysis was performed using GraphPad Prism 8.01 (Graphpad Software, La Jolla, CA, USA). Data are presented as median \pm interquartile range. A Wilcoxon matched-pairs signed rank test was performed to compare two groups. Comparisons between more than two groups were made using Friedman tests with multiple comparisons. A p-value $\leq 0.05$ was considered statistically significant. 

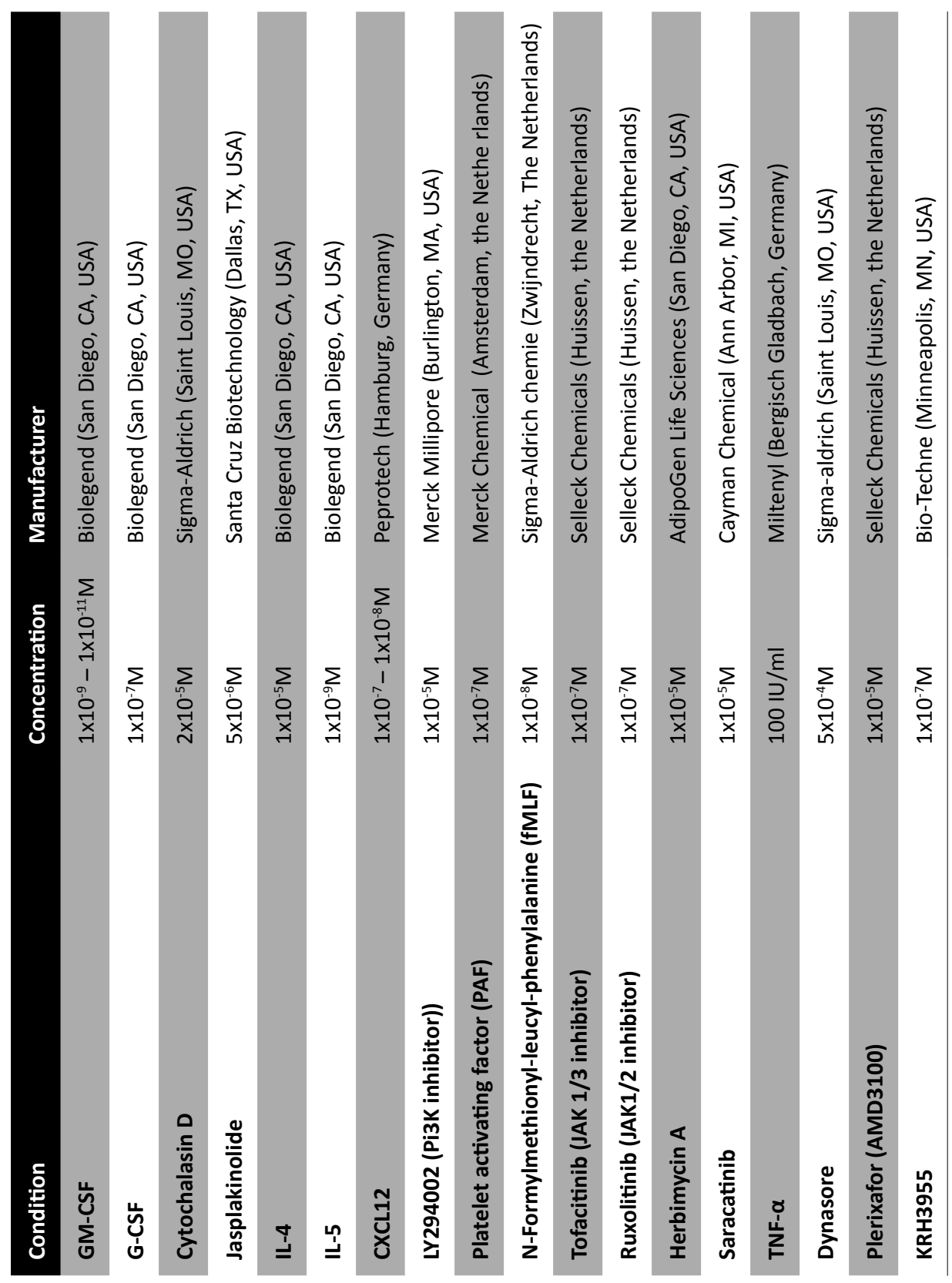

Table 1. The different conditions used in several experiments. 


\section{Results}

\section{CXCR4 is lowly expressed on freshly isolated neutrophils}

Freshly isolated total leukocytes from healthy volunteers were stained with anti-CXCR4 or an IgG1 isotype and expression of both antibodies was measured by flow cytometry. The expression of CXCR4 was determined on different leukocytes, which were identified on the basis of FSC-A and SSC-A characteristics (monocytes, lymphocytes and granulocytes) or on the basis of auto-fluorescence characteristics (distinction of neutrophils from eosinophils). This showed that freshly isolated neutrophils compared to other leukocytes had a low expression of CXCR4 on the surface membrane (Figure 1A). Next, by measuring samples with the ImageStream, we visualized surface CXCR4 expression on neutrophils (recognized based on CD16 staining) and lymphocytes (recognized based on CD3 staining) which remarkably revealed that besides a low CXCR4 expression in neutrophils, this expression seemed to be focal in contrast to a more homogeneous surface expression on lymphocytes (Figure 1B). Finally we compared the total CXCR4 protein in PBMCs (monocytes and lymphocytes) and granulocytes which similarly showed a higher expression on PBMCs compared to granulocytes (Figure 1C), indicating that CXCR4 is overall lower expressed on fresh neutrophils compared to lymphocytes.

A

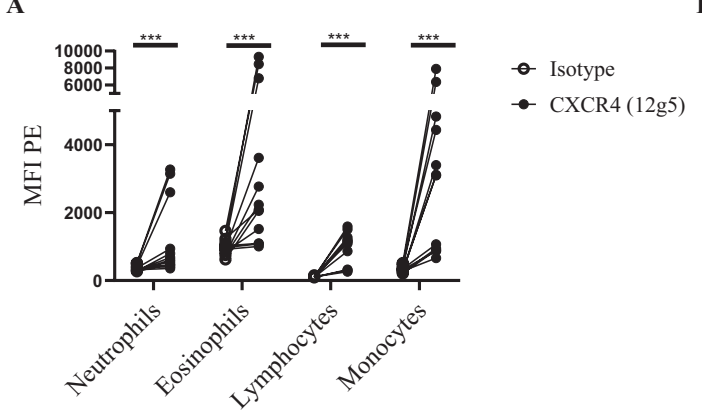

B

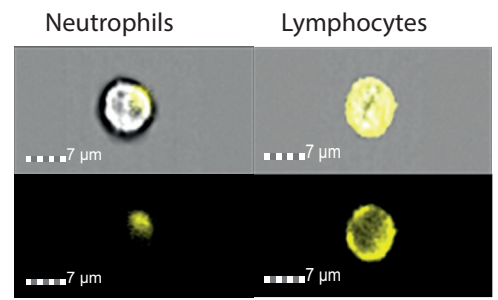

C PBMC Donor 1 Donor 2

42

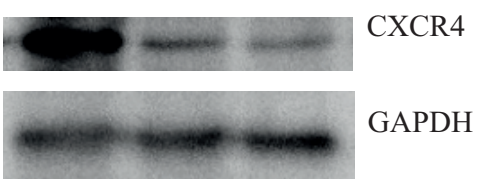

Figure 1: CXCR4 expression on freshly isolated leukocytes. A. Median fluorescence intensity of CXCR4 (12G5) on the surface of different freshly isolated leukocytes was compared with isotype expression $(n=12)$. Cells were gated based on FSC/SSC characteristics and auto fluorescence (eosinophils). B. A representative image of CXCR4 expression on the surface membrane is shown for neutrophils (left) and lymphocytes (right). Image was acquired on the ImageStream with 60x magnification. C. A representative western blot is shown for total CXCR4 protein and GAPDH expression on freshly isolated PBMCs and on granulocytes of two donors. 


\section{Freshly isolated neutrophils respond to CXCL12}

Next, we found that neutrophils, stimulated with CXCL12, showed an obvious increase in cytosolic free $\mathrm{Ca}^{2+}\left(\left[\mathrm{Ca}^{2+}\right]_{\mathrm{i}}\right)$, despite the low membrane expression of CXCR4 (Figure $2 \mathrm{~A}$ and 2D). Since, many chemokines are known to be promiscuous for their receptors, we made sure that this increase in $\left[\mathrm{Ca}^{2+}\right]_{i}$ is initiated by CXCR4 specifically by showing inhibition by the CXCR4 specific antagonists AMD3100 ${ }^{19}$ and $\mathrm{KRH}_{3955^{20}}$. Indeed both CXCR4 antagonists abolished the CXC12 induced increase in $\left[\mathrm{Ca}^{2+}\right]_{\mathrm{i}}$, without an effect on PAF induced change in $\left[\mathrm{Ca}^{2+}\right]_{\mathrm{i}}$ (Figure $2 \mathrm{~B}-\mathrm{D}$ ). Moreover, stimulating freshly isolated neutrophils with CXCL12 led to a very modest phosphorylation of ERK1/2 (Figure 2E) as this p-ERK signal was much less strong compared to stimulation with fMLF (Figure $2 \mathrm{E})$, demonstrating that the ERK response to CXCL12 is only minor. Furthermore, CXCL12 was able to prime ROS production after co-stimulation with $\mathrm{fMLF}$, albeit to a lesser extent than PAF (Figure 2F). Additionally, stimulation of neutrophils with CXCL12 also led to minor activation of neutrophils in terms of expression of activation markers detectable on the cell surface (Supplementary Figure 1). Surprisingly, when we tested chemotaxis to CXCL12 of freshly isolated neutrophils and 6 hours in vitro aged neutrophils, we did not find any suggestion of chemotaxis or even chemokinesis in response to this chemokine (Supplementary Figure 2). Neutrophils did, however, show a strong chemotaxis towards fMLF.

\section{CXCL12 induced increase in $\left[\mathrm{Ca}^{2+}\right]_{i}$ in neutrophils is completely and specifically inhibited by GM-CSF}

Most neutrophils responded to CXCL12 with a normal $\mathrm{Ca}^{2+}$-response, but remarkably in certain donors not all cells responded (Figure 2D). Therefore, we tested the hypothesis whether an additional priming step with GM-CSF, a known priming agent for granulocytes ${ }^{21}$, would increase the number of neutrophils that respond to CXCL12. Much to our surprise, and completely against our expectation, pre-incubating neutrophils with GM-CSF completely abolished the change in $\left[\mathrm{Ca}^{2+}\right]_{i}$, induced by CXCL12 (compare Figure $3 \mathrm{~A}$ with Figure $3 \mathrm{~B}$ ). We tested whether this inhibition was specific for GM-CSF or that other granulocyte priming cytokines, such as TNF- $\alpha^{22}$ or G-CSF ${ }^{23}$ induced a similar inhibitory effect on CXCR4 function. Both TNF- $\alpha$ and G-CSF were not affecting CXCL12 induced change in $\left[\mathrm{Ca}^{2+}\right]_{\mathrm{i}}$ (Figure $3 \mathrm{C}$ ). Vice versa, GM-CSF did not inhibit an increase $\left[\mathrm{Ca}^{2+}\right]_{i}$ induced by other $\mathrm{G}$-protein coupled receptors (Supplementary Figure 3A-C) such as for PAF (Figure 3B) or for fMLF (data not shown).

Next, we compared CXCR4 expression on the surface membrane of freshly isolated unstimulated neutrophils with neutrophils that were stimulated with GM-CSF (Figure 3D). These experiments showed that CXCR4 expression was not affected. Also, the total CXCR4 protein quantity did not decrease after stimulating neutrophils with GM-CSF (Figure 3E). Therefore, it is likely that CXL12 is still able to bind CXCR4, but its effect on $\left.\left[\mathrm{Ca}^{2+}\right]_{\mathrm{i}}\right)$, is inhibited by GM-CSF induced signaling. 
A
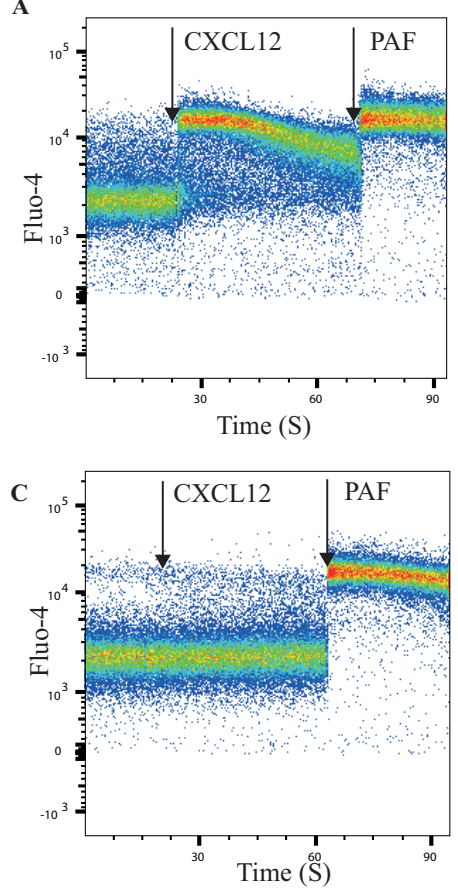

$\mathbf{E}$

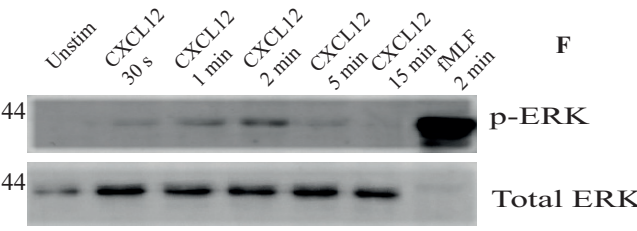

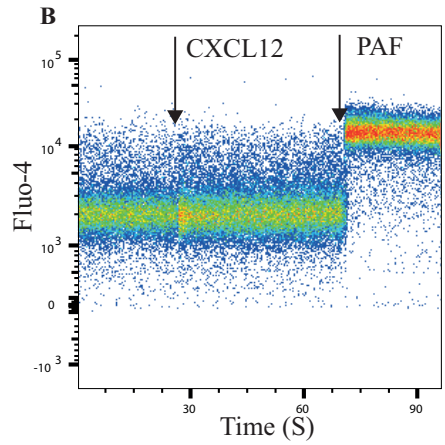

D
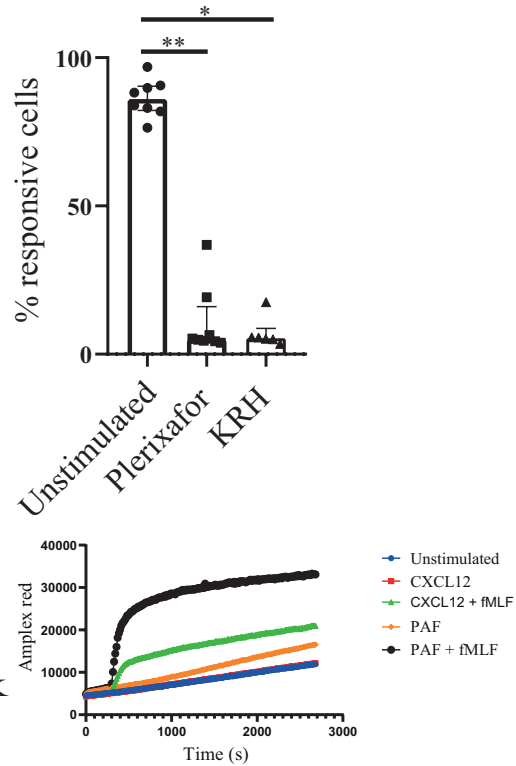

Figure 2. CXCL12 induced $\mathrm{Ca}^{2+}$-influx in freshly isolated neutrophils.

A. Freshly isolated leukocytes were loaded with FLUO-4 and $\mathrm{Ca}^{2+}$-influx was determined on gated neutrophils after stimulation with $100 \mathrm{nM} \mathrm{CXCL12}$ and after $100 \mathrm{nM}$ PAF. A representative FACS plot is shown. B. Total FLUO-4 loaded leukocytes were pre-incubated with $10 \mu \mathrm{M}$ AMD3100 (plerixafor) for 15 minutes at $37^{\circ} \mathrm{C}$ and $\mathrm{Ca}^{2+}$-influx was similarly measured after stimulation with $100 \mathrm{nM} \mathrm{CXCL12}$ and 100 nM PAF. C. Total leukocytes were pre-incubated with $100 \mathrm{nM}$ KRH3955 for 15 minutes at $37^{\circ} \mathrm{C}$ and $\mathrm{Ca}^{2+}$ influx was similarly measured after stimulation with $100 \mathrm{nM} \mathrm{CXCL12}$ and $100 \mathrm{nM}$ PAF. D. The amount of FLUO-4 positive neutrophils after CXCL12 stimulation (\%responsive neutrophils) was quantified prior to stimulation with plerixafor $(n=8), K R H 3955(n=6)$ or on unstimulated neutrophils (15 minutes on $37^{\circ} \mathrm{C}$ only, $n=8$ ). Median with interquartile range is indicated. A Wilcoxon matched-pairs signed rank test was performed ${ }^{*} P \leq 0.05 * * P \leq 0.01$. E. Representative western blot is shown for $p$-ERK and total ERK expression in freshly isolated neutrophils at different time points after stimulation with $100 \mathrm{nM}$ CXCL12 or 2 minutes after $10 \mathrm{nM}$ fMLF. F. ROS-production was measured in freshly isolated neutrophils by 
measuring amplex red fluorescence on a fluoro-luminometer during at least 1 hour: blue line represents unstimulated neutrophils, red after $100 \mathrm{nM} \mathrm{CXCL12,} \mathrm{green} 100 \mathrm{nM} \mathrm{CXCL12} \mathrm{followed} \mathrm{by} 10 \mathrm{nM}$ fMLF, orange $10 \mathrm{nM}$ PAF and black $10 \mathrm{nM}$ PAF followed by $10 \mathrm{nM}$ fMLF. The lines are formed by the medians of three independent experiments.

A

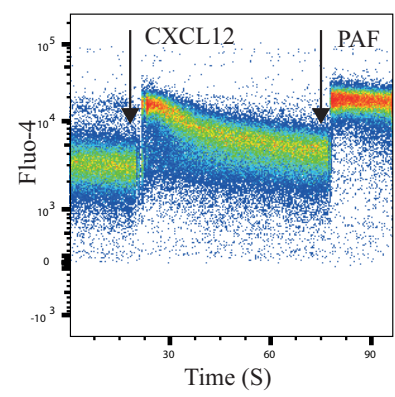

D

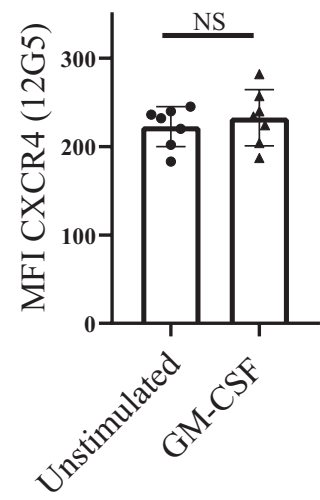

B

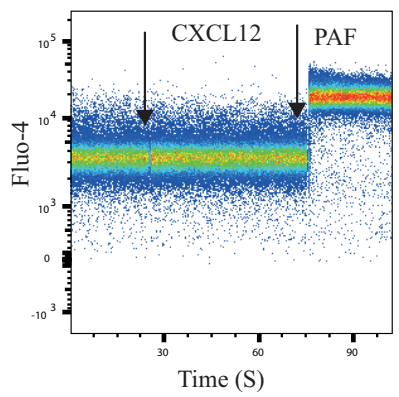

$\mathbf{E}$
C

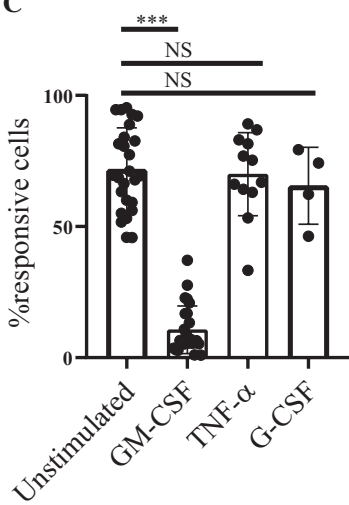

GMCSF

and CXCL12

42

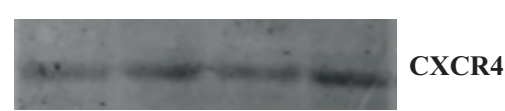

26

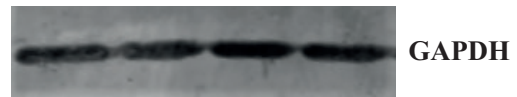

Figure 3. The effect of GM-CSF on CXCR4 signaling and expression.

A. Priorly unstimulated neutrophils were FLUO-4 loaded and $\mathrm{Ca}^{2+}-$ influx was measured after stimulation with $100 \mathrm{nM}$ CXCL12 and $100 \mathrm{nM}$ PAF. A representative FACS plot is shown. B. Neutrophils were stimulated with $1 \mathrm{nM} \mathrm{GM}$-CSF for 30 minutes at $37^{\circ} \mathrm{C}$ and $\mathrm{Ca}^{2+}$-influx was similarly measured after stimulation with $100 \mathrm{nM}$ CXCL12 and $100 \mathrm{nM}$ PAF. A representative FACS plot is shown. C. The percentage of responsive neutrophils to $100 \mathrm{nM}$ CXCL12 was quantified for priorly unstimulated neutrophils $(n=24)$ or neutrophil stimulated with $1 \mathrm{nM} \mathrm{GM-CSF}$ (30 minutes, $\mathrm{n}=24), 100 \mathrm{IU} / \mathrm{ml}$ TNF- $\alpha$ (15 minutes, $\mathrm{n}=12$ ) or $100 \mathrm{nM} \mathrm{G-CSF}$ (30 minutes, $\mathrm{n}=4$ ). D. MFI of CXCR4 was measured on unstimulated neutrophils or neutrophils priorly stimulated with $1 \mathrm{nM} \mathrm{GM-CSF} \mathrm{(30} \mathrm{minutes,} n=7)$. E. A representative western blot is shown for total CXCR4 protein and GAPDH on freshly isolated unstimulated neutrophils or neutrophils that were stimulated with $100 \mathrm{nM} \mathrm{CXCL12} \mathrm{(15} \mathrm{minutes),} 1 \mathrm{nM} \mathrm{GM-CSF} \mathrm{(30} \mathrm{minutes)} \mathrm{or} 1 \mathrm{nM}$ GM-CSF (30 minutes) followed by $100 \mathrm{nM}$ CXCL12 (15 minutes). C-D. The median with interquartile range is indicated. A Wilcoxon matched-pairs signed rank test was performed. NS. Not significant. ${ }^{* * * P} \leq 0.001$. 


\section{GM-CSF blocks CXCL12 induced $\left[\mathrm{Ca}^{2+}\right]_{i}$ through the activation of JAK2}

Next, we tested whether the effect of GM-CSF on CXCR4 was dose dependent. Low doses of GM-CSF ( $\leq 1 \mathrm{pM}$ ) did not inhibit CXCL12 induced $\mathrm{Ca}^{2+}$ signal. Hereafter, a dose dependent inhibition was induced by GM-CSF with maximum at $1 \mathrm{nM}$ (Figure 4A). The inhibitory effect of GM-CSF on CXCR4 took at least 5-15 minutes of pre-incubation before it became significant (Figure 4B). Adding GM-CSF directly before stimulation with CXCL12 did not inhibit the increase in $\left[\mathrm{Ca}^{2+}\right]_{i}($ Figure $4 \mathrm{~B}, \mathrm{t}=0)$. This timeframe fits with the minimum time that is needed to phosphorylate juxtamembranous tyrosine kinases associated with the ß-chain of the GM-CSF receptor ${ }^{24}$. This implied that the effect of GM-CSF was induced by the common ß-chain, which is shared with the IL-3 and IL-5 receptor ${ }^{25}$. Indeed, when eosinophils were pre-incubated with IL-5 and GM-CSF, a similar inhibition of CXCR4 was induced (Supplementary Figure $3 \mathrm{D}-\mathrm{F}$ ).

A

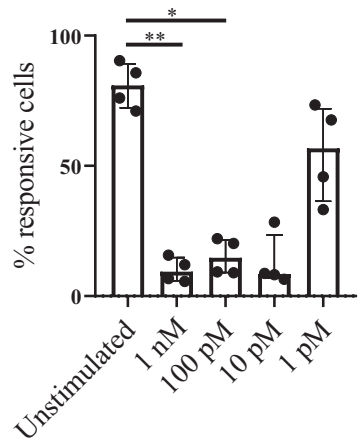

D
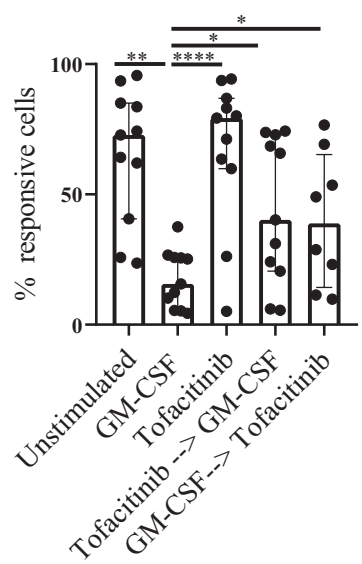

B
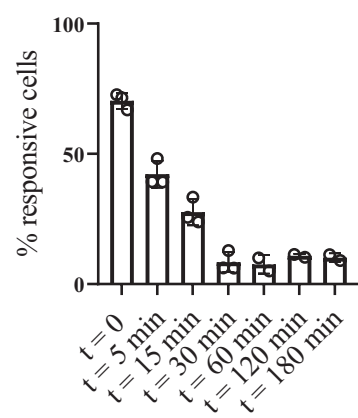

$\mathbf{E}$
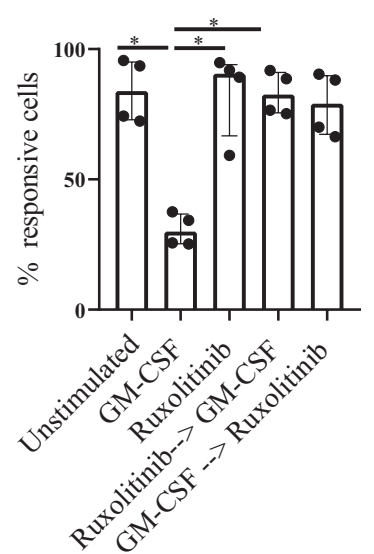

C
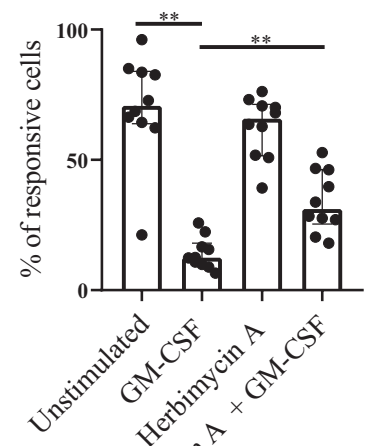

Figure 4. GM-CSF inhibits CXCL12 induced $\mathrm{Ca}^{2+}$-influx via activation of tyrosine kinases JAKs.

A. Percentage of FLUO-4 positive neutrophils after stimulation with $10 \mathrm{nM} \mathrm{CXCL12}$ was determined in unstimulated neutrophils $(n=4)$ and neutrophils that were priorly stimulated with GM-CSF at concentrations ranging from $1 \mathrm{nM}$ to $1 \mathrm{pM}$ for 30 minutes. B. The percentage responsive neutrophils after stimulation with $10 \mathrm{nM} \mathrm{CXCL12}$ was determined after priorly stimulating neutrophils with $1 \mathrm{nM}$ GM-CSF for a different amount of time $(n=3)$, ranging from directly after $(t=0)$ to $t=180$ minutes. $C$. The 
percentage responsive neutrophils was determined after activation with $10 \mathrm{nM} \mathrm{CXCL12} \mathrm{in} \mathrm{unstimulated}$ neutrophils $(n=10)$ or after pre-incubating neutrophils with GM-CSF only (100 pM, 30 minutes), Herbimycin-A only (10 $\mu \mathrm{M}, 15$ minutes) or GM-CSF (100 pM, 30 minutes) followed by Herbimycin-A $(10 \mu \mathrm{M}, 15$ minutes). D. Percentage responsive neutrophils was determined after activation with $10 \mathrm{nM}$ CXCL12 in unstimulated neutrophils $(n=11)$ or neutrophils that were pre-incubated with GM-CSF (100 pM, 30 minutes), Tofacitinib ( $100 \mathrm{nM}, 30$ minutes), Tofacitinib ( $100 \mathrm{nM}, 30$ minutes) followed by GM-CSF (100 pM, 30 minutes) or vice versa $(n=8)$. E. This was similarly done as is described in $\mathbf{D}$ but with $100 \mathrm{nM}$ Ruxolitinib $(n=4)$. Median with interquartile range is indicated. A Wilcoxon matched-pairs signed rank test was performed. Only statistically significant results are indicated. ${ }^{*} \mathrm{P} \leq 0.05^{* *} \mathrm{P} \leq 0.01$ and ${ }^{* * *} \mathrm{P} \leq$ 0.001 .

The importance of these tyrosine kinases in the GM-CSF-induced inhibition came from experiments applying the general tyrosine kinase inhibitor herbimycin-A. When, neutrophils were pre-incubated with herbimycin-A, the inhibitory effect of GM-CSF on CXCR4 was largely eliminated (Figure 4C). More specifically, inhibition of JAKs with either tofacitinib ${ }^{26}$ or ruxolitinib ${ }^{27}$ also largely reduced the inhibitory effect of GM-CSF on CXCR4 (Figure 4D-E). Surprisingly, stimulating neutrophils with GM-CSF first during at least 30 minutes, followed by incubating neutrophils with either JAK inhibitors also restored CXL12 induced increase in $\left[\mathrm{Ca}^{2+}\right]_{i}$ (Figure 4D-E). This implied that the effect of GM-CSF on the CXCR4 receptor was reversible. Downstream signaling induced by GM-CSF such as GRK3 or PI3K did not seem to be involved, because inhibition of these proteins by specific inhibitors did not reduce the inhibitory effect of GM-CSF (Supplementary Figure 4A-B). Finally, also inhibition of Src by saracatinib did not reverse GM-CSF effect on CXCR4 (Supplementary Figure 4C).

\section{GM-CSF-induced modification of the actin cytoskeleton blocks CXCL12 induced $\left[\mathrm{Ca}^{2+}\right]_{i}$}

Next, we set out experiments to find out how GM-CSF specifically inhibited CXCR4 signaling. We first tested the hypothesis that CXCR4 expression was not affected by GM-CSF by using AF647 labeled CXCL12. Addition, of this labeled agonist showed a small increase of fluorescence on neutrophils irrespective of GM-CSF stimulation or not, while this increase was not seen in neutrophils previously incubated with AMD3100 or KRH3955 (Supplementary Figure 5).

We next hypothesized that CXCR4 receptor mobility might play a role, since this is important for CXCR4 activation ${ }^{28,29,30}$. A first set of experiments tested whether the inhibition of actin polymerization, caused by GM-CSF, would abrogate its inhibitory effect on CXCR4. Indeed, the effect of GM-CSF was reversed significantly when neutrophils were stimulated with cytochalasin $D^{31}$, a potent inhibitor of actin polymerization, (Figure 5A). Vice versa, when we stimulated neutrophils with jasplakinolide, an agent that causes actin polymerization ${ }^{32}$, a similar inhibition of CXCL12 induced $\mathrm{Ca}^{2+}$-influx was seen as with stimulation with GM-CSF which indicated that actin polymerization might be important (Figure 5B). Also incubating neutrophils with dynasore, an inhibitor of clathrin dependent endocytosis ${ }^{33}$, strongly reduced CXCL12 induced $\mathrm{Ca}^{2+}$-influx (Figure 5C) while it did not inhibit the $\mathrm{Ca}^{2+}$ influx of PAF, fMLF, IL-8 or Gro- $\alpha$ (data not shown). All these findings, strongly suggested that the mobility of the CXCR4 receptor specifically was important for its function and that this is reduced by the effect of GM-CSF. 
A
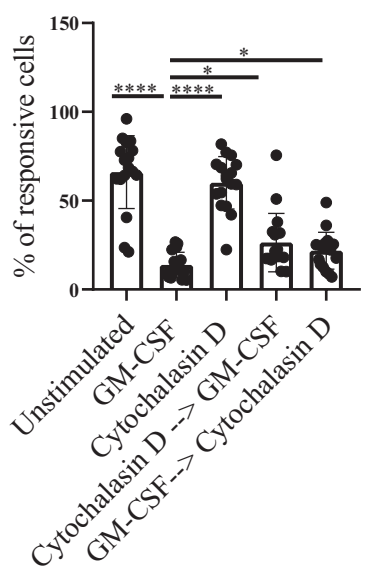

B
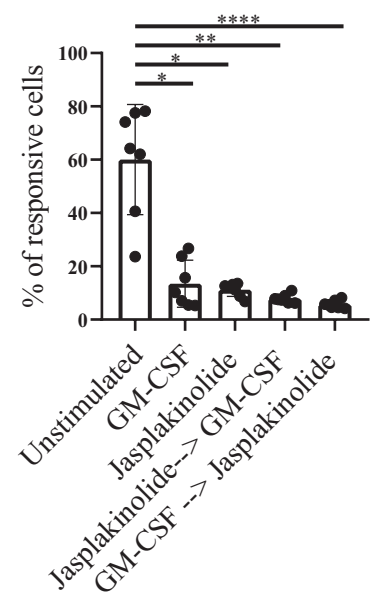

C
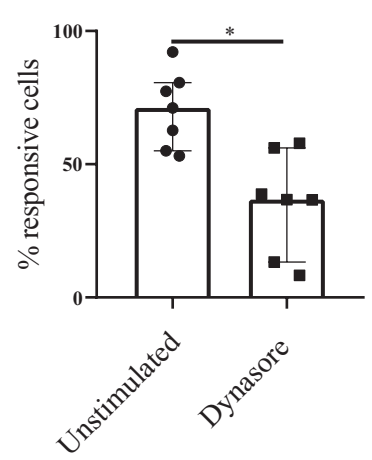

Figure 5. The role of the actin cytoskeleton on GM-CSF induced inhibition of CXCR4.

A. Percentage of FLUO-4 positive neutrophils after stimulation with $10 \mathrm{nM} \mathrm{CXCL12}$ was determined in unstimulated neutrophils $(n=7)$ and neutrophils that were priorly stimulated with GM-CSF (100 pM,

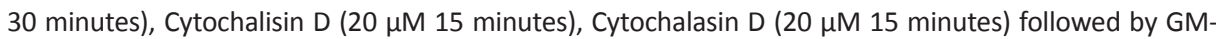
CSF (100 pM, 30 minutes) or vice versa. B. This was similarly done as described in A but with $5 \mu \mathrm{M}$ Jasplakinolide (15 minutes) instead of Cytochalasin D. C. Percentage of FLUO-4 positive neutrophils after activation with $10 \mathrm{nM} \mathrm{CXCL12}$ was determined in unstimulated neutrophils (only 15 minutes at $37^{\circ} \mathrm{C}$ ) and neutrophils that were priorly stimulated with $500 \mu \mathrm{M}$ Dynasore ( 15 minutes at $37^{\circ} \mathrm{C}$ ). Median with interquartile range is indicated. A Wilcoxon matched-pairs signed rank test was performed. Only statistically significant results are indicated. $* P \leq 0.05 * * P \leq 0.01$ and $* * * * P \leq 0.0001$.

\section{Discussion}

To our knowledge, this is the first study in which the functionality, signaling and expression of CXCR4 on freshly isolated human neutrophils were studied in depth. This is also the first study in which it is demonstrated that a short term ( $5-30 \mathrm{~min}$ ) incubation with GM-CSF (or more specifically the common ß-chain of the receptors for GM-CSF, IL-3 and IL-5) was able to rapidly block $\mathrm{Ca}^{2+}$-signaling caused by CXCL12 binding to CXCR4. A similar report was published several years ago in which longterm stimulation ( 24 hours) with GM-CSF (but also IL-4) resulted in CXCR4 inhibition ${ }^{34}$. However, our findings are different from this earlier report, because it is: i. more rapid (after 15 minutes), ii. it is unique for GM-CSF (not IL-4), iii. it is not induced by receptor internalization (Figure 3D and supplementary Figure 5) and iv. GRK3 seems not be involved (Supplementary Figure 4A).

Our finding might be clinically relevant, because CXCR4 is important in neutrophil trafficking from and to the bone marrow ${ }^{6}$. Therefore, GM-CSF induced inhibition of CXCR4 might be able to cause acute egress of neutrophils from the bone marrow to the blood, similar as is seen with plerixafor ${ }^{35}$. This is exactly what was found in early phase-1 trial showing that already 4 hours after the first dose of GM-CSF in cancer patients 
a doubling of neutrophil count in the blood was observed ${ }^{36}$. This finding cannot be caused by an increase of (emergency) granulopoiesis, as this would take more time ${ }^{37}$. Instead, it is possible that this rapid release of neutrophils to the blood is caused by the inhibition of CXCR4 receptor on (segmented) neutrophils. This acute effect of GMCSF could be used in several disorders (e.g. neutropenia caused by chemotherapy, congenital neutropenias etc.) in order to rapidly mobilize neutrophils from the bone marrow to the blood and hence decrease the chances of infectious complications. However, this effect should first be (re)evaluated in another study performed in healthy volunteers with treatments with recombinant GM-CSF.

Freshly isolated neutrophils respond to CXCL12 with an increase in $\left[\mathrm{Ca}^{2+}\right]_{i}$, which is accompanied by a very minor increase in expression of activation markers (Supplementary Figure 1). We also showed the ability of CXCL12 to prime the reactive oxygen species (ROS) in response to fMLF (Figure 2). However, we did not find any suggestions that CXCL12 caused chemotaxis or significant chemokinesis in freshly isolated neutrophils (Supplementary Figure 2). It is, therefore, tempting to speculate that only low amounts of receptor surface expression are needed to cause $\mathrm{Ca}^{2+}$-influx, whereas for other receptor functionality like chemotaxis, a higher receptor expression and or signal strength are needed. Since CXCR4 expression increases in time ${ }^{10,11}$, it is possible that other signaling mechanisms are merely revealed in aged neutrophils. Indeed, in contrast to our finding, in vitro aged neutrophils (18 hours!) responded to CXCL12 with a chemotactic response ${ }^{11}$. It is possible that neutrophils need to age even longer (at least 18 hours) in order to respond to CXCL12 in this context. Also phosphorylation of ERK induced in CXCL12 is much more evident in aged neutrophils compared to freshly isolated neutrophils ${ }^{11}$. This also indicated that higher receptor expression is needed to activate several signaling mechanisms.

GM-CSF inhibited CXCL12-induced increase in $\left[\mathrm{Ca}^{2+}\right]_{i}$ via the activation of JAK2 (Figure 4). This seems surprising as G-CSF is not able to block CXCR4, whereas it is also able to phosphorylate and activate JAKs in other cells ${ }^{38}$. However, in contrast to GM-CSF which has been shown to selectively activate JAK2 in human neutrophils ${ }^{39}$, G-CSF is not able to phosphorylate JAK2 in mature human neutrophils ${ }^{40}$. It is, therefore, likely that GM-CSF induced inhibition of CXCR4 is initiated through the activation of JAK2. This hypothesis is supported by the finding that Ruxolitinib (a selective JAK1/2 inhibitor) ${ }^{27}$ is more potent in the restoration of GM-CSF induced inhibition of CXCR4 (Figure 4) than Tofacitinib (a selective JAK1/3 inhibitor with less selectivity against JAK2 $)^{41}$. Alternatively, it is possible that activation of JAKs through GM-CSF leads to other signaling mechanisms downstream when compared to signaling through the G-CSF receptor, but this should be evaluated further.

Inhibition of actin polymerization by pretreatment with cytochalasin $D$ reversed the effect of GM-CSF inhibition on CXCR4 implying the importance of this mechanism. This was supported by the finding that jasplakinolide also inhibited the CXCL12 induced $\mathrm{Ca}^{2+}$ influx in neutrophils. Both findings suggest that modification of the actin cytoskeleton (polymerization) caused by GM-CSF ${ }^{42}$ is involved in the inhibition of CXCR4.This putative modification of the actin cytoskeleton might result in the inhibition of CXCR4 receptor dimerization $^{28}$ or receptor internalization ${ }^{29}$, since both mechanisms were found to be selectively important in CXCR4 signaling. A logical next step would be to study receptor 
dimerization by FRET assays in neutrophils before and after GM-CSF stimulation in order to test whether CXCL12 is still able to induce dimerization of CXCR4. Alternatively, single particle tracking ${ }^{43}$ allows the detection of CXCR4 internalization or clustering after stimulation with CXCL12 which might also be inhibited as a result of GM-CSF activation.

Finally, we showed that other trimeric G-protein coupled receptors: CXCR1/2, FPR1/2 and the PAF receptor were not inhibited by the effect of GM-CSF. This suggests that whatever the mode of action for GM-CSF is, its inhibitory effect seems specific for CXCR4. A specific inhibitory effect of a cytokine receptor on a chemokine receptor in neutrophils is not unprecedented, as TNF $\alpha$ has also been described to specifically inhibit the action of CXCR2 receptor including a change in $\left[\mathrm{Ca}^{2+}\right]_{i}^{44}$. However, the inhibitory effect of TNF $\alpha$ was accomplished by a different mechanism, because TNF $\alpha$ induced enhanced shedding of CXCR2 receptor through the action of serine protease(s) resulting in decreased CXCR2 expression on neutrophils ${ }^{44}$. We, however, did not find a difference in CXCR4 expression or a difference in fluorescent CXCL12 binding after stimulation with GM-CSF which strongly argues against a down regulation of CXCR4 on the cell membrane.

In conclusion, we have shown that $\mathrm{CXCL} 12$ induced $\left[\mathrm{Ca}^{2+}\right]_{i}$ in neutrophils is rapidly and specifically inhibited by activation of the common ß-chain of the GM-CSF receptor. Therefore, our study demonstrates that cross-talk between cytokine and chemokine receptors drastically changes their signaling. This implies that modulation of cytokines by currently developed biologicals should be evaluated in the proper inflammatory context. 


\section{References}

1. Griffith, J. W., Sokol, C. L. \& Luster, A. D. Chemokines and chemokine receptors: positioning cells for host defense and immunity. Annu. Rev. Immunol. 32, 659-702 (2014).

2. Oberlin, E. et al. The CXC chemokine SDF-1 is the ligand for LESTR/fusin and prevents infection by T-cell-line-adapted HIV-1. Nature 382, 833-5 (1996).

3. Förster, R. et al. Intracellular and surface expression of the HIV-1 coreceptor CXCR4/fusin on various leukocyte subsets: rapid internalization and recycling upon activation. J. Immunol. 160, 1522-31 (1998).

4. Bleul, C. C., Wu, L., Hoxie, J. A., Springer, T. A. \& Mackay, C. R. The HIV coreceptors CXCR4 and CCR5 are differentially expressed and regulated on human T lymphocytes. Proc. Natl. Acad. Sci. U. S. A. 94, 1925-30 (1997).

5. MA, M., N, A. \& AR, B. CXCR4 and CCR7: Two Eligible Targets in Targeted Cancer Therapy. Cell Biol. Int. 40, (2016).

6. Eash, K. J., Means, J. M., White, D. W. \& Link, D. C. CXCR4 is a key regulator of neutrophil release from the bone marrow under basal and stress granulopoiesis conditions. Blood 113, 4711-9 (2009).

7. Hernandez, P. A. et al. Mutations in the chemokine receptor gene CXCR4 are associated with WHIM syndrome, a combined immunodeficiency disease. Nat. Genet. 34, 70-4 (2003).

8. Martin, C. et al. Chemokines acting via CXCR2 and CXCR4 control the release of neutrophils from the bone marrow and their return following senescence. Immunity 19, 583-93 (2003).

9. Suratt, B. T. et al. Role of the CXCR4/SDF-1 chemokine axis in circulating neutrophil homeostasis. Blood 104, 565-71 (2004).

10. Nagase, H. et al. Cytokine-mediated regulation of CXCR4 expression in human neutrophils. J. Leukoc. Biol. 71, 711-7 (2002).

11. Weisel, K. C. et al. Modulation of CXC chemokine receptor expression and function in human neutrophils during aging in vitro suggests a role in their clearance from circulation. Mediators Inflamm. 2009, 790174 (2009).

12. De Filippo, K. \& Rankin, S. M. CXCR4, the master regulator of neutrophil trafficking in homeostasis and disease. Eur. J. Clin. Invest. 48 Suppl 2, e12949 (2018).

13. Crump, M. P. et al. Solution structure and basis for functional activity of stromal cell-derived factor-1; dissociation of CXCR4 activation from binding and inhibition of HIV-1. EMBO J. 16, 6996-7007 (1997).

14. Putney, J. W. \& Tomita, T. Phospholipase C signaling and calcium influx. Adv. Biol. Regul. 52, 152-164 (2012).

15. Tsai, F.-C. et al. A polarized Ca2+, diacylglycerol and STIM1 signalling system regulates directed cell migration. Nat. Cell Biol. 16, 133-44 (2014).

16. Kutsuna, $\mathrm{H}$. et al. Actin reorganization and morphological changes in human neutrophils stimulated by TNF, GM-CSF, and G-CSF: the role of MAP kinases. Am. J. Physiol. Cell Physiol. 286, C55-64 (2004).

17. Ferguson, S. S. et al. Role of beta-arrestin in mediating agonist-promoted G protein-coupled receptor internalization. Science 271, 363-6 (1996).

18. van Grinsven, E. et al. Immature Neutrophils Released in Acute Inflammation Exhibit Efficient Migration despite Incomplete Segmentation of the Nucleus. J. Immunol. 202, 207217 (2019). 
19. Hendrix, C. W. et al. Safety, pharmacokinetics, and antiviral activity of AMD3100, a selective CXCR4 receptor inhibitor, in HIV-1 infection. J. Acquir. Immune Defic. Syndr. 37, 1253-62 (2004).

20. Murakami, T. et al. The novel CXCR4 antagonist KRH-3955 is an orally bioavailable and extremely potent inhibitor of human immunodeficiency virus type 1 infection: comparative studies with AMD3100. Antimicrob. Agents Chemother. 53, 2940-8 (2009).

21. Coffer, P. J. \& Koenderman, L. Granulocyte signal transduction and priming: cause without effect? Immunol. Lett 57, 27-31 (1997).

22. Lloyds, D., Brindle, N. P. \& Hallett, M. B. Priming of human neutrophils by tumour necrosis factor-alpha and substance $\mathrm{P}$ is associated with tyrosine phosphorylation. Immunology $\mathbf{8 4}$, 220-6 (1995).

23. Balazovich, K. J., Almeida, H. I. \& Boxer, L. A. Recombinant human G-CSF and GM-CSF prime human neutrophils for superoxide production through different signal transduction mechanisms. J. Lab. Clin. Med. 118, 576-84 (1991).

24. Okuda, K. et al. Granulocyte-macrophage colony-stimulating factor, interleukin-3, and steel factor induce rapid tyrosine phosphorylation of p42 and p44 MAP kinase. Blood 79, 2880-7 (1992).

25. Broughton, S. E. et al. The betac receptor family - Structural insights and their functional implications. Cytokine 74, 247-258 (2015).

26. Furuya, M. Y. et al. Tofacitinib inhibits granulocyte-macrophage colony-stimulating factorinduced NLRP3 inflammasome activation in human neutrophils. Arthritis Res. Ther. 20, 196 (2018).

27. Gunawan, A. S. et al. Ruxolitinib, a potent JAK1/JAK2 inhibitor, induces temporary reductions in the allelic burden of concurrent CSF3R mutations in chronic neutrophilic leukemia. Haematologica 102, e238-e240 (2017).

28. Ge, B. et al. Single-molecule imaging reveals dimerization/oligomerization of CXCR4 on plasma membrane closely related to its function. Sci. Rep. 7, 16873 (2017).

29. E, B. et al. CXCR4 Signaling Is Controlled by Immobilization at the Plasma Membrane. Biochim. Biophys. Acta 1863, (2016).

30. DeNies, M. S., Rosselli-Murai, L. K., Schnell, S. \& Liu, A. P. Clathrin Heavy Chain Knockdown Impacts CXCR4 Signaling and Post-translational Modification. Front. cell Dev. Biol. 7, 77 (2019).

31. M, S. Action of Cytochalasin D on Cytoskeletal Networks. J. Cell Biol. 92, (1982).

32. Bengtsson, T., Orselius, K. \& Wetterö, J. Role of the actin cytoskeleton during respiratory burst in chemoattractant-stimulated neutrophils. Cell Biol. Int. 30, 154-63 (2006).

33. Macia, E. et al. Dynasore, a cell-permeable inhibitor of dynamin. Dev. Cell 10, 839-50 (2006).

34. Wang, J. et al. Role of tyrosine phosphorylation in ligand-independent sequestration of CXCR4 in human primary monocytes-macrophages. J. Biol. Chem. 276, 49236-43 (2001).

35. Hendrix, C. W. et al. Pharmacokinetics and safety of AMD-3100, a novel antagonist of the CXCR-4 chemokine receptor, in human volunteers. Antimicrob. Agents Chemother. 44, 1667-73 (2000).

36. Lieschke, G. J. et al. Phase I study of intravenously administered bacterially synthesized granulocyte-macrophage colony-stimulating factor and comparison with subcutaneous administration. Cancer Res. 50, 606-14 (1990).

37. Wood, A. J. J., Lieschke, G. J. \& Burgess, A. W. Granulocyte Colony-Stimulating Factor and Granulocyte-Macrophage Colony-Stimulating Factor. N. Engl. J. Med. 327, 28-35 (1992). 
38. Marino, V. J. \& Roguin, L. P. The granulocyte colony stimulating factor (G-CSF) activates Jak/STAT and MAPK pathways in a trophoblastic cell line. J. Cell. Biochem. 103, 1512-1523 (2008).

39. Al-Shami, A., Mahanna, W. \& Naccache, P. H. Granulocyte-Macrophage Colony-stimulating Factor-activated Signaling Pathways in Human Neutrophils. J. Biol. Chem. 273, 1058-1063 (1998).

40. Shimoda, K. et al. G-CSF Induces Tyrosine Phosphorylation of the JAK2 Protein in the Human Myeloid G-CSF Responsive and Proliferative Cells, but Not in Mature Neutrophils. Biochem. Biophys. Res. Commun. 203, 922-928 (1994).

41. Clark, J. D., Flanagan, M. E. \& Telliez, J.-B. Discovery and Development of Janus Kinase (JAK) Inhibitors for Inflammatory Diseases. J. Med. Chem. 57, 5023-5038 (2014).

42. Gomez-Cambronero, J., Horn, J., Paul, C. C. \& Baumann, M. A. Granulocyte-macrophage colony-stimulating factor is a chemoattractant cytokine for human neutrophils: involvement of the ribosomal p70 S6 kinase signaling pathway. J. Immunol. 171, 6846-55 (2003).

43. Brandsma, A. M. et al. Mechanisms of inside-out signaling of the high-affinity IgG receptor FcyRI. Sci. Signal. 11, eaaq0891 (2018).

44. Asagoe, K. et al. Down-regulation of CXCR2 expression on human polymorphonuclear leukocytes by TNF-alpha. J. Immunol. 160, 4518-25 (1998). 
A

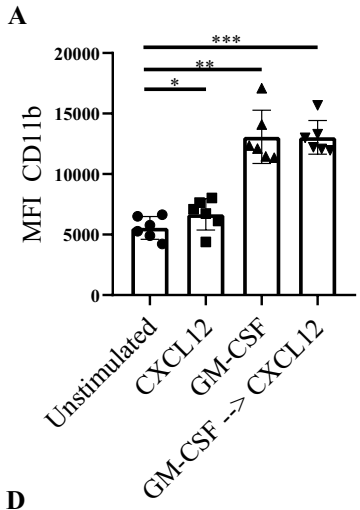

D
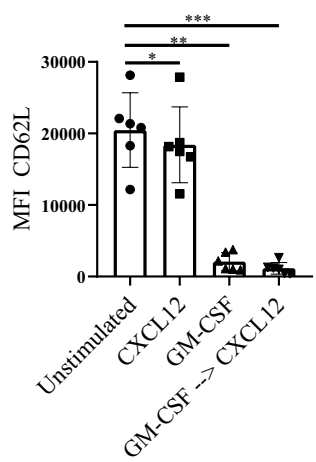

B

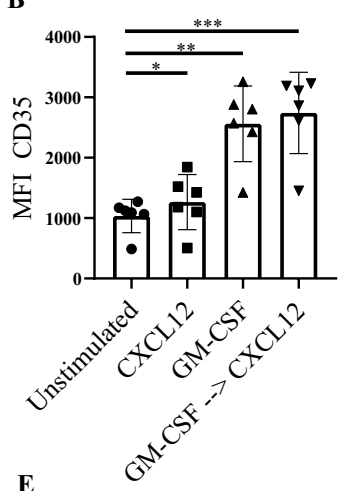

$\mathbf{E}$

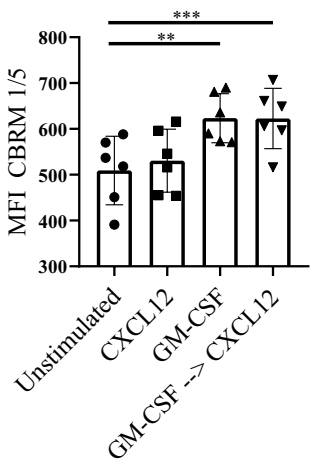

C
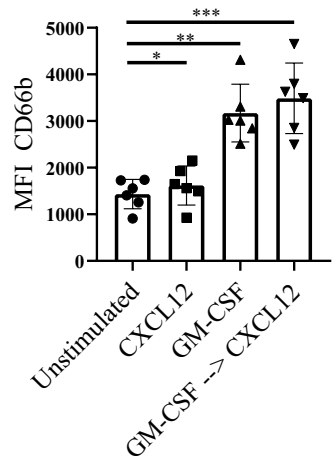

Supplementary Figure 1. CXCL12 induces a small activation in freshly isolated neutrophils.

Median fluorescence intensity of different activation markers was determined on neutrophils in unstimulated whole blood $\left(15\right.$ minutes at $37^{\circ} \mathrm{C}$ ), or whole blood that was stimulated with $50 \mathrm{nM} \mathrm{CXCL12}$ $\left(15\right.$ minutes at $\left.37^{\circ} \mathrm{C}\right), 1 \mathrm{nM} \mathrm{GM}-\mathrm{CSF}\left(45\right.$ minutes at $\left.37^{\circ} \mathrm{C}\right)$ or with $\mathrm{GM}-\mathrm{CSF}\left(1 \mathrm{nM}, 30\right.$ minutes at $\left.37^{\circ} \mathrm{C}\right)$ followed by $50 \mathrm{nM} \mathrm{CXCL12}\left(15\right.$ minutes at $\left.37^{\circ} \mathrm{C}\right)$. Red blood cells were thereafter lysed and total leukocytes were stained with multiple antibodies. Neutrophils were gated based on FSC/SSC and CD66b and CD16 expression. A. CD11b, B. CD35, C. CD66b. D. CD62L and E. CD11b (clone CBRM1/5 which only recognizes the active epitope of MAC1 complex). Median with interquartile range is indicated. A Wilcoxon matchedpairs signed rank test was performed. Only statistically significant results are indicated. ${ }^{*} \mathrm{P} \leq 0.05$ $* * \mathrm{P} \leq 0.01$ and $* * * \mathrm{P} \leq 0.001$. 
A

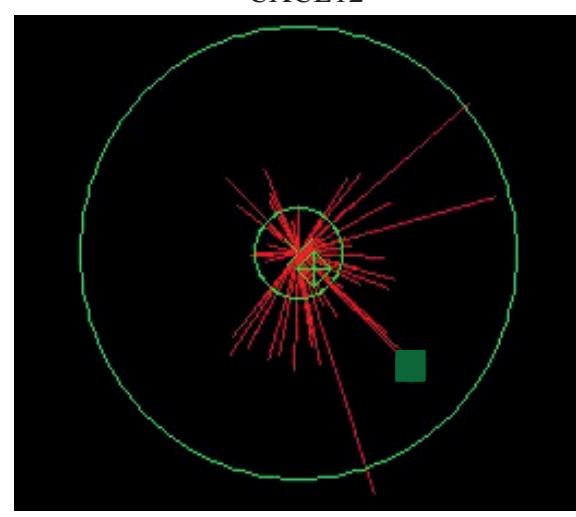

Buffer

C

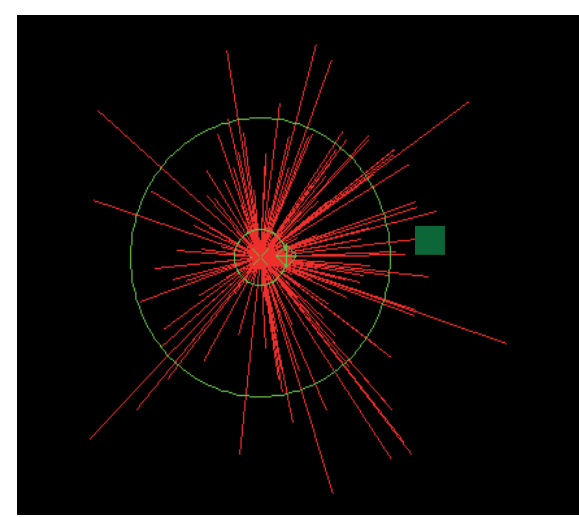

Buffer
B

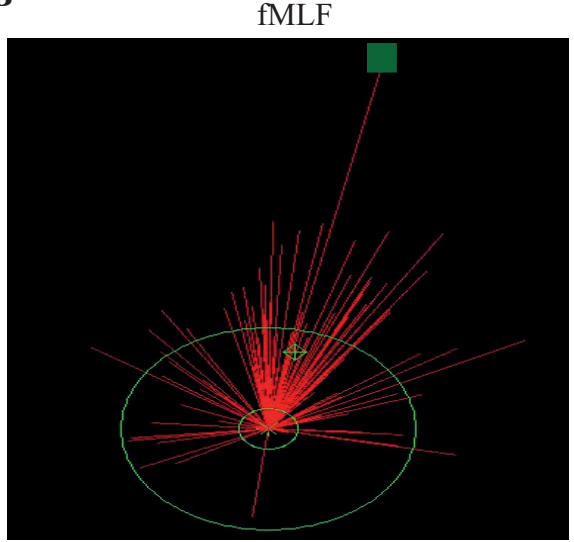

Buffer

D

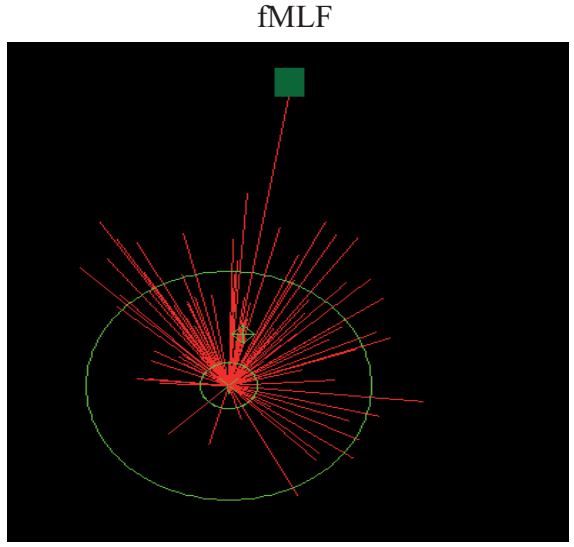

Buffer

Supplementary Figure 2. Neutrophil chemotaxis to CXCL12 and fMLF.

Isolated neutrophils were placed in the center of a $10 \mu$-Slide chemotaxis chamber inside a fibrin gel. The chemotactic agent (CXCL12 or fMLF) was added in the compartment above the neutrophils, while buffer was added below. Movement (direction and distance) of each cell is indicated in a red line. The average distance and direction is indicated by a green square.

A. Isolated neutrophils were aged in vitro for 6 hours and thereafter placed in the chemotaxis assay with $100 \mathrm{nM}$ CXCL12 above or buffer below. B. similar as in A, but neutrophils were now placed with $10 \mathrm{nM}$ fMLF. C. in vitro aged neutrophils (6 hours) were stimulated with $1 \mathrm{nM} \mathrm{GM-CSF}$ for at least 30 minutes and thereafter placed in the chemotaxis assay with $100 \mathrm{nM} \mathrm{CXCL12} \mathrm{(above)} \mathrm{or} \mathrm{buffer} \mathrm{(below).} \mathrm{D.} \mathrm{similar}$ as C, but neutrophils were now placed with $10 \mathrm{nM} \mathrm{fMLF}$. 
A
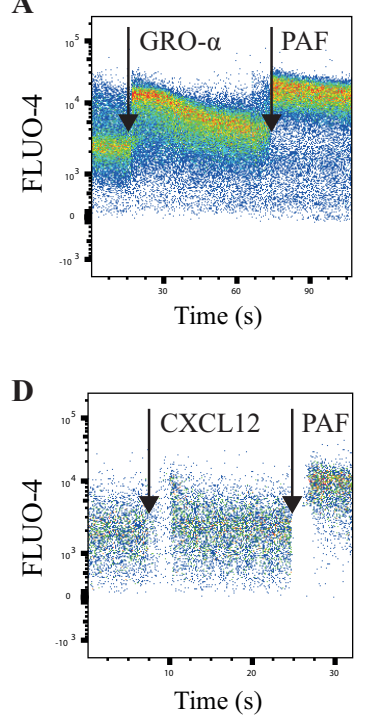

B

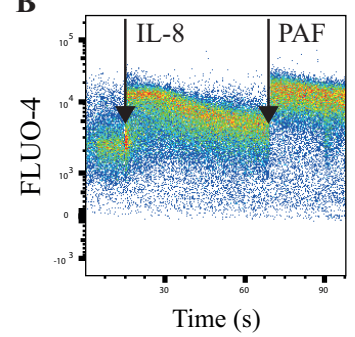

$\mathbf{E}$

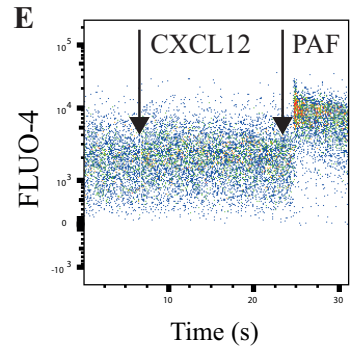

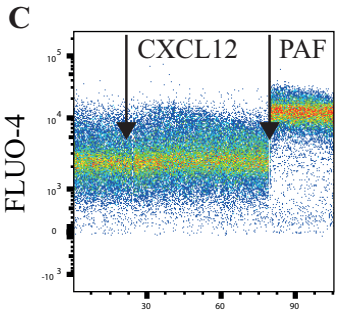

Time (s)

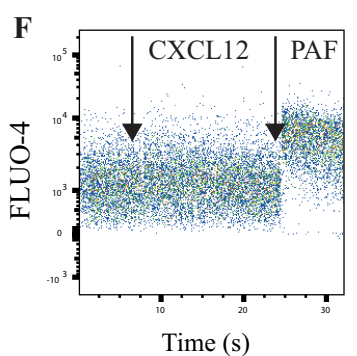

Supplementary Figure 3. CXCL12 induced $\mathrm{Ca}^{2+}$-influx is uniquely inhibited by the common B-chain.

A-C. Total leukocytes were pre-incubated with $1 \mathrm{nM}$ GM-CSF for 30 minutes and thereafter $\mathrm{Ca}^{2+}$-influx was determined on neutrophils after stimulation with $10 \mathrm{nM}$ Gro- $\alpha$ (A), $10 \mathrm{nM} \mathrm{IL-8} \mathrm{(B)} \mathrm{or} 10 \mathrm{nM}$ CXCL12 (C). D. CXCL12 induced $\mathrm{Ca}^{2+}$-influx is also seen in eosinophils. E. This CXCL12 induced $\mathrm{Ca}^{2+}$-influx in eosinophils is similarly inhibited by $1 \mathrm{nM}$ GM-CSF or F. $1 \mathrm{nM} \mathrm{IL-5.}$ 
A
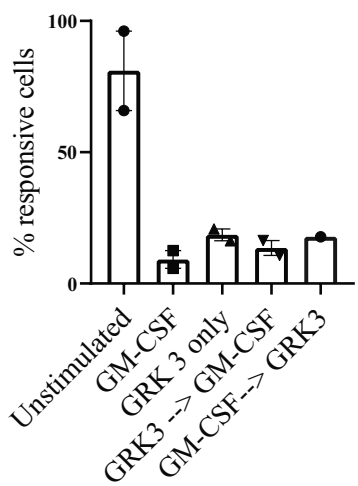

B
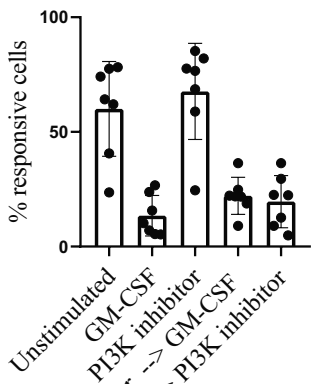

(i)
C
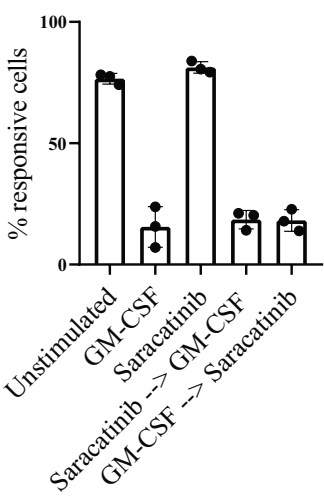

Supplementary Figure 4. Failed GM-CSF inhibitors.

A. Percentage of FLUO-4 positive neutrophils after stimulation with $10 \mathrm{nM} \mathrm{CXCL12} \mathrm{was} \mathrm{determined} \mathrm{in}$ unstimulated neutrophils $(n=2)$ and neutrophils that were priorly stimulated with GM-CSF (100 pM, 30 minutes), GRK1/3 inhibitor: CMPD101 (100 $\mu \mathrm{M} 15$ minutes) or GM-CSF (100 pM, 30minutes) followed by GRK1/3 inhibitor ( $100 \mu \mathrm{M}, 15$ minutes) or vice versa. This was similarly done, but with the B. Pi3K inhibitor (LY294002, $\mathrm{n}=7,10 \mu \mathrm{M}$ for 15 minutes) and C. with Saracitinib ( $\mathrm{n}=2,10 \mu \mathrm{M}$ for 15 minutes). Median with interquartile range is also indicated.

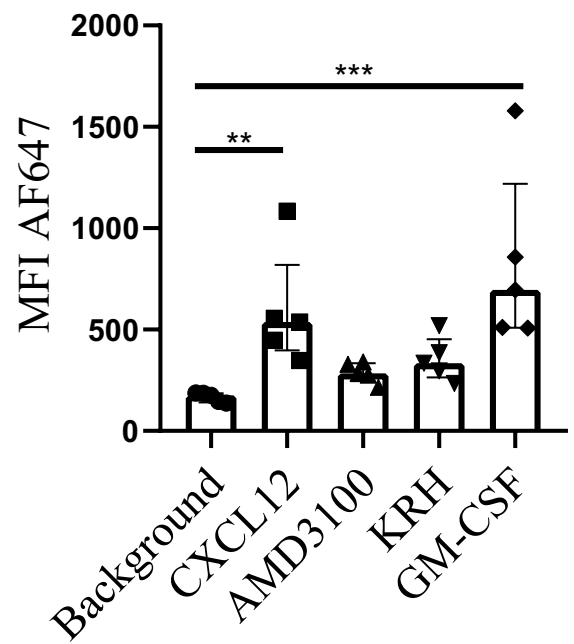

\section{Supplementary Figure 5. Median fluorescent intensity of AF647 labeled CXCL12.}

Total leukocytes were stimulated with 10 nM AF647 labeled CXCL12 (Almac group, Souderton, PA, USA) for 10 minutes at room temperature. Thereafter cells were washed and MFI of AF647 was determined on unstimulated neutrophils or neutrophils that were pre-incubated with AMD3100, KRH395 or GM$\operatorname{CSF}(n=5)$. Median with interquartile range is also indicated. A Friedman test with multiple comparisons was performed. Only statistically significant results are indicated. ${ }^{* *} \mathrm{P} \leq 0.01$ and ${ }^{* * *} \mathrm{P} \leq 0.001$. 



\section{Part II}

Eosinophil kinetics 


\section{Chapter 6}

\section{Immunological and hematological effects of IL-5(R $\alpha)$ targeted therapy: an overview}

M. Hassani ${ }^{1}$, L. Koenderman ${ }^{1}$

1. Department of Respiratory Medicine, Laboratory of Translational Immunology, University Medical Centre Utrecht, Utrecht, the Netherlands. 


\section{Abstract}

IL-5 is an important cytokine for priming and survival of mature eosinophils, and for proliferation and maturation of their progenitors. Hence, IL-5(R $\alpha)$ targeting will be increasingly used in diseases where eosinophils are the key immune effector cells such as eosinophilic asthma (EA), hyper eosinophilic syndrome (HES), eosinophilic esophagitis (EE) and eosinophilic granulomatosis with polyangitis (EGPA). Therefore, several neutralizing monoclonal antibodies directed against IL-5 (mepolizumab and reslizumab) and its receptor IL-5R (benralizumab) have found or will find their way to the clinic. While the clinical effect of these drugs has been extensively investigated and reviewed, the understanding of the underlying immunological and hematological mechanisms remains less clear.

This review will discuss the translational outcomes of treatment with these monoclonal antibodies in humans in order to shed light on the mechanisms underlying the main immunological and hematological findings from these clinical trials in humans. 


\section{Introduction}

Therapies targeted on the IL-5 pathway are a good extension in the treatment of patients with severe eosinophilic asthma. ${ }^{1}$ This therapeutical approach has also shown promising results in the treatment of nasal polyps, hyper eosinophilic syndrome, eosinophilic granulomatosis with polyangiitis and other hyper eosinophilic disorders ${ }^{2-4}$. For patients with severe eosinophilic asthma treatment with IL-5 targeting drugs results in a lower rate of exacerbations and a decrease in use of glucocorticoids. However, the quality of life and pre-bronchodilator FEV1 hardly improves in a clinically relevant way. ${ }^{1,5-13}$ In addition, only half of the patients with eosinophilic granulomatosis with polyangiitis reached a remission. ${ }^{3}$ The lack of a complete clinical response in both diseases are difficult to understand as long as some important immunological and hematological issues of the therapy remain to be established. One of the key questions is whether eosinophils that remain in the body during IL-5 targeted therapy are an intrinsically different non-responsive subset or residual 'normal' cells, supporting the view that IL-5 is not critical in human eosinophilopoiesis. A third possibility is that residual eosinophils are found because of under dosing of the monoclonal antibodies such as recently suggested. ${ }^{3,14,15}$ To gather translational data on IL-5 inhibition: a systemic literature search in PubMed was performed on October 2017 with the following query: (mepolizumab OR nucala OR reslizumab OR benralizumab OR cinqaero OR "anti-il-5" OR "anti-il-5r" OR "anti il-5" OR "anti il 5" OR "anti interleukin 5" OR "anti-interleukin 5" OR "anti-interleukin-5"). This query yielded 749 articles that were assessed for relevance and validity on the basis of title and abstract firstly and on full text secondly.
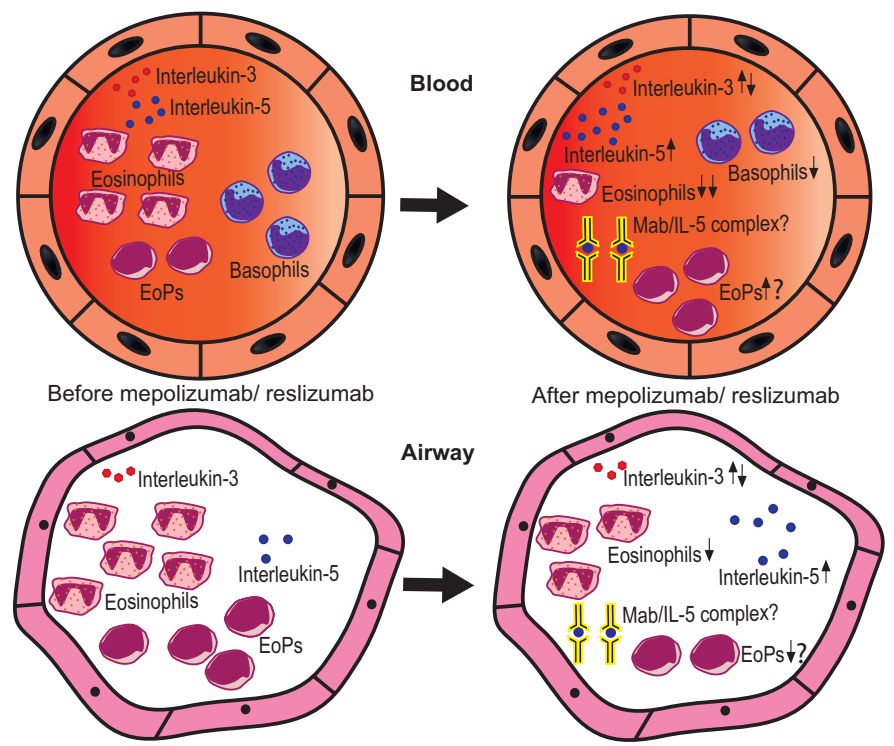

\section{Graphical abstract.}

IL-5 targeted therapy results in an attenuation of eosinophils and basophils in blood and an attenuation of eosinophils and EoPs in the air-ways. Plasma and sputum levels of IL-5 increase, possibly due to the formation of immune complexes between IL-5 and anti-IL-5. 


\section{The function of interleukin-5 in homeostasis}

\section{Receptor of IL-5 and its signal transduction}

Interleukin-5 is a cytokine which is produced as a dimer and secreted by multiple cells such as Th2 cells, mast cells, ILC2 cells and eosinophils. ${ }^{16}$ It binds to the IL-5-specific $\alpha$ subunit- interleukin-5R $\alpha$ (CD125)- that is part of a heterodimeric receptor with the common $\beta$ subunit (CD131). This latter subunit is shared with the heterodimeric IL-3 receptor (CD123) and GM-CSF receptor (CD116). ${ }^{17}$ The common $\beta$ subunit doesn't express any ligand binding site but confers high-affinity ligand binding to intracellular signaling. ${ }^{18}$ Upon binding of IL-5 to its receptor, juxtamembranous tyrosine kinases phosphorylate the $\beta c$ receptor upon which at least three major signaling pathways are activated: JAK/ STAT, MAPK and PI-3K. ${ }^{19}$ All three pathways eventually lead to rapid reprogramming of gene expression, and a plethora of cellular responses ranging from proliferation of eosinophil progenitors to priming of cytotoxicity by mature cells. ${ }^{20}$ Interestingly, IL-5 with a charge reversal mutation at position $12(\mathrm{E} 12 \mathrm{~K})^{21}$ and GM-CSF with a similar mutation at position 21 (E21R), ${ }^{22}$ which are important for receptor binding, fail to stimulate tyrosine phosphorylation but can still affect survival. ${ }^{21}$ This finding reveals the existence of two distinct mechanisms of receptor activation, one of which is $\alpha$-chain specific.

\section{Expression of IL-5 receptors}

In humans the effects of IL-5 are restricted to basophils and eosinophils. ${ }^{23}$ The expression of IL-5R $\alpha$ on basophils is 3-fold lower compared to mature eosinophils and their differentiation is not dependent on this cytokine ${ }^{24}$. Therefore, the receptor is best characterized in the context of eosinophils. The receptor is both expressed on mature eosinophils and their progenitors including the eosinophil lineage specific myeloblast (CD34+ and IL-5R $\alpha+$ cells). ${ }^{25}$ Since stimulation of CD34+/IL-5R $\alpha+$ cells only produces eosinophils, it is yet not clear at what stage the IL-5R $\alpha$ is upregulated on basophils. ${ }^{25}$ In tissue the level of IL-5R $\alpha$ on eosinophils is lower compared to blood eosinophils. ${ }^{26,}$

${ }^{27}$ It seems plausible that the receptor is shed after migration to the tissue, because the amount of soluble IL-5R $\alpha$ is increased in tissue. This hypothesis is supported by the finding that IL-5R $\alpha$ is shed from the surface of healthy control eosinophils in vitro upon interaction with IL-5. This is, probably due to proteolytic cleavage. ${ }^{28}$ Whether IL$5 \mathrm{R} \alpha$ expression is also found on type 2 innate lymphoid cells (ILC2) is still a subject of debate, because the results in literature are inconsistent. ${ }^{29,} 30$

\section{The role of IL-5R in differentiation and proliferation}

IL-5 stimulates eosinophil colony formation in bone marrow samples in vitro. IL-3 and GM-CSF can also give rise to eosinophil colonies in vitro, but unlike the other cytokines IL-5 is the most eosinophil specific. ${ }^{31,32}$ The concentrations of IL-3 and GM-CSF in vitro needs to be tenfold higher in order to create eosinophils from marrow mononuclear cells instead of solely neutrophils or monocytes. ${ }^{32}$ The formation of basophil colonies is also stimulated by IL-5 in HL-60 cells albeit to a lesser extent. ${ }^{32,33}$

In vivo, the situation is more complex. Murine experiments show a central role of IL-5 for reactive eosinophilia rather than differentiation per se, as IL-5 knockout mice do contain mature eosinophils in peripheral blood. They, however, do not exhibit eosinophilia after parasite infection. In culture systems of human CD34+ progenitor 
cells IL-5 only induces transient proliferation and maturation of eosinophil precursors, which also suggests that IL-5 is a relatively late-acting factor for eosinophil proliferation rather than a factor for early differentiation. ${ }^{34}$ All these experiments imply that other factors drive differentiation of eosinophil precursors and IL-5 is a major growth factor for IL-5R expressing cells, at least in mice. ${ }^{34}$

\section{The IL-5R and survival}

In vitro the presence of IL-5 and GM-CSF increases mature eosinophil survival up to 10 days by inhibition of apoptosis. ${ }^{35-37}$ The apoptotic eosinophils are normally recognized and phagocytosed by macrophages or to a lesser extent by small-airway epithelial cells in a process generally referred to as efferocytosis. ${ }^{38,39}$ In vivo the average circulatory lifespan of eosinophils was estimated to be between 11 and 63 hours. This large range is probably due to ex vivo manipulation of cells, ${ }^{40}$ the use of toxic labe ${ }^{40-42}$ and the difference in kinetics between homeostasis and pathological conditions. ${ }^{43}$ The effect of IL-5 on these kinetics is unknown, but a clear negative correlation between eosinophil apoptosis and sputum IL-5 levels was found in asthmatic patients, which suggests that IL-5 is involved in inhibition of apoptosis in eosinophils in vivo. ${ }^{44}$ Another important receptor involved in eosinophil survival is Siglec-8. In contrast to IL-5, GM-CSF and IL-3, activation of Siglec-8 by Mab or glycan ligands induced cell death. ${ }^{45}$ Interestingly this ROS dependent regulated cell death only occurred after priming of cells with IL-5, GM-CSF or IL-33. ${ }^{46}$ This finding suggests that steady state and resident eosinophils are less susceptible for Siglec- 8 induced cell death. ${ }^{45}$

\section{IL-5 targeted therapy: How and what are the options or alternatives?}

Mepolizumab and reslizumab are both humanized monoclonal antibodies (Mab) that bind to and block the function of circulating IL-5 and consequently prevent binding of IL-5 to its receptor. Mepolizumab is an IgG1 kappa Mab registered to be administered via a subcutaneous injection with a fixed dose of $100 \mathrm{mg}$ whereas reslizumab is a IgG4 kappa Mab which is registered to be injected intravenously with a weight adjusted concentration of $3 \mathrm{mg} / \mathrm{kg} .{ }^{47}$ Benralizumab is an IgG1 kappa antibody directed against the IL-5R $\alpha$. The published data imply that benralizumab can completely deplete eosinophils and their (late) bone marrow progenitors by induction of antibody-dependent cell-mediated cytotoxicity (ADCC) executed by natural killer-cells and/or macrophages both in vitro and in non-human primates in vivo. ${ }^{24}$ Since phase 3 trials show promising results, this drug will probably be approved soon in the treatment of severe eosinophilic asthma as well. ${ }^{1}$ Omalizumab is a humanized monoclonal antibody that binds to $C \varepsilon 3$ of IgE and blocks this protein from binding to its receptor that is located on mast cells and basophils. ${ }^{48}$ The required doses are dependent on body weight and serum levels of IgE. Since IgE is important in the Th2 allergic immune response, omalizumab is prescribed for patients with severe allergic asthma with high levels of aero-allergen specific IgE. ${ }^{49}$ Both anti-IL-5 and anti-IgE target Th2 mediated inflammation and are therefore eligible for treating patients with eosinophilic and allergic asthma. A systematic review of available literature has shown that mepolizumab and omalizumab have similar therapeutic effects and tolerability in responsive patients with severe asthma. ${ }^{50}$ Since eosinophilic inflammation isn't necessarily mediated through the "classical" IgE pathway, ${ }^{51}$ anti-IL-5 seems to be favorable in all patients with signs of eosinophilic inflammation, but a direct clinical comparison hasn't been performed yet. 


\section{Key therapeutic effects of IL-5 inhibition in eosinophilic driven diseases}

All asthma trials have in common that the effect of IL-5(R) targeted therapy primarily leads to a decrease in disease exacerbations and steroid dependence rather than a direct effect on asthma characteristics such as changes in BHR and lung function. ${ }^{1}$ For chronic rhinosinusitis with polyposis both reslizumab and mepolizumab led to an improvement in symptoms for most, but not all patients. ${ }^{52,53}$ Besides this clinical effect, surgery was no longer required in $30 \%$ of patients treated with mepolizumab. ${ }^{54}$ For eosinophilic esophagitis, anti-IL-5 didn't lead to improvement of symptoms or histological remission. ${ }^{55}$ Treating patients with hyper eosinophilic syndrome (HES) with mepolizumab resulted in a lower dependence of prednisolone in less than half of the patients. ${ }^{4}$ Similarly, less than half of the eosinophilic granulomatosis and polyangitis (EGPA) patients treated with mepolizumab reached complete remission. ${ }^{3}$ Finally in atopic dermatitis mepolizumab did not lead to any clinical significant results, even though eosinophils may play a role in this disease..$^{56}$

\section{Interleukin-5 targeted therapy: difference between mice and men}

There are key differences between murine and human data regarding pathogenesis of asthma and IL-5 targeted therapy. ${ }^{23,57-59}$ Therefore this review focusses on human data rather than murine data.

\section{Effect of anti-IL5(R) on eosinophil and basophil numbers in peripheral blood and tissues}

There hasn't been a study performed that directly compared mepolizumab and reslizumab in a randomized controlled clinical trial. However, the results in the separate trials were comparable when it comes to reducing the number of circulating eosinophils (table 1). ${ }^{5-11}$ Still, it was speculated that in a certain group of patients with steroid unresponsive asthma, the fixed lower concentration of mepolizumab (100 mg) might be insufficient to reduce the number of eosinophils in the tissues. It was even speculated based on preliminary studies that this low concentration might lead to formation of immune complexes between IL- 5 and mepolizumab potentially activating tissue eosinophils. ${ }^{14,15}$ It was indeed found that lower concentrations of antibody were associated with lower reduction of both circulating and tissue eosinophils (Pavord et al 2012 table 1). ${ }^{6,60}$ However, no data to date have directly measured the formation of immune complexes under these conditions and it remains to be established whether immune complexes are a worry in IL-5 targeted therapy.

Benralizumab depletes circulating eosinophils completely, likely through ADCC, although in some patients a small number circulatory cells still remained. ${ }^{10,12,13,61}$ However, the number of mucosal and/or submucosal airway eosinophils was less affected by the treatment when compared to the effect on the number of circulating cells. ${ }^{13,62}$ It is too early to speculate how to interpret this counterintuitive finding, but the concept of the parallel presence of homeostatic and inflammatory eosinophils in the airways of eosinophilic asthmatics might shed some light on the tissue dwelling cells in the future. ${ }^{63}$ Next to altering eosinophil levels, $750 \mathrm{mg}$ of mepolizumab also reduced the number of basophils in the tissue and bone marrow, although this reduction was less pronounced compared to eosinophils. ${ }^{64} \mathrm{It}$ is, therefore, likely that basophils rely on different cytokines such as IL-3. ${ }^{65,66}$

The effect of mepolizumab on mast cells in tissue remains uncertain, since inconsistent 
results have been published. ${ }^{24,64,67}$ Evidence that mast cells express the IL-5R $\alpha$ receptor has only been provided in vitro in very limited number of studies. ${ }^{68}$

Surprisingly, even though the expression of IL-5R $\alpha$ is 3 -fold lower on basophils, still a complete depletion of also basophils in peripheral blood is seen after treatment with benralizumab. ${ }^{24,62}$ This suggests that even though basophils rely on IL-3, a low membrane expression of IL-5R $\alpha$ can already induce clearance through ADCC.

\section{IL-5 is not essential for eosinophil and basophil differentiation and production during homeostasis.}

It is now tempting to speculate why in response to all above mentioned therapies a small amount of eosinophils can still be found in sputum or bronchial tissue in patients with asthma. 5, 6, 8, 13, 62 Also treatment of patients with eosinophilic esophagitis with mepolizumab, induced a reduction in the number of eosinophils in circulation and in esophageal tissue when compared to placebo but did not lead to complete depletion of the cells. Interestingly, the number of homeostatic eosinophils in the duodenum was not altered by mepolizumab even after treatment with a high concentration of mepolizumab (1500 mg). ${ }^{69}$ It is possible that IL-5R $\alpha$ expression can be redundant by co-expression of IL-3 and GM-CSF receptors and the presence of sufficient amounts of IL-3 or GM-CSF. It might even be that certain tissue eosinophils lose their IL-5R $\alpha$ such as described for cells found in the BAL after segmental allergen challenge. ${ }^{70}$ To date, the data in the literature are not yet sufficient to reach such conclusion, but it is relevant to emphasize that tissue eosinophils are found in homeostasis without any indication that IL-5 is driving this response. The IL-5R $\alpha$ expression on these resident eosinophils of the duodenum seems lower, compared to circulating eosinophil . ${ }^{69}$ This is not likely caused by homologous desensitization as it is completely unclear what the source of IL-5 would be under these conditions, but ILC- 2 cells are a putative source. ${ }^{71}$

\section{Effect of anti-IL5(receptor)Mab's on eosinophil progenitors in and outside the bone marrow}

Eosinophilopoiesis takes place in the bone marrow particularly under homeostasis. Under pathological conditions such as found in severe asthma, eosinophil progenitors (CD34+ and IL-5R $\alpha$ positive progenitors) have also been found in tissue and blood. This supports the hypothesis that extramedullary eosinophilopoiesis can take place. ${ }^{72}$ In fact, in severe prednisolone dependent asthma the amount of eosinophil-lineage-committed progenitor cells in sputum was over 700-fold higher compared to healthy controls, supporting a role of extramedullary hematopoiesis in eosinophil driven disease. ${ }^{73}$

In the bone marrow mepolizumab (administered $750 \mathrm{mg}$ intravenously) reduced both the mature and late immature eosinophils (myelocytes and metamyelocytes) significantly compared to placebo while the number of EoPs (early progenitors) wasn't attenuated in blood or bone marrow. ${ }^{74}$ It can be hypothesized that IL-5 mainly influences eosinophil proliferation and maturation of relatively late progenitors rather than the reduction of early progenitors themselves. ${ }^{74}$ However, the relative amount of late immature and mature eosinophils in the bone marrow was still high after two months of therapy (mean of $1.9 \%$ and $1.1 \%$ respectively). This lack of complete depletion of relatively late progenitors might be due to a role of other cytokines, such as IL-3 and GM-CSF. ${ }^{74}$ Indeed, treatment with GM-CSF also leads to eosinophilia. ${ }^{75}$ 


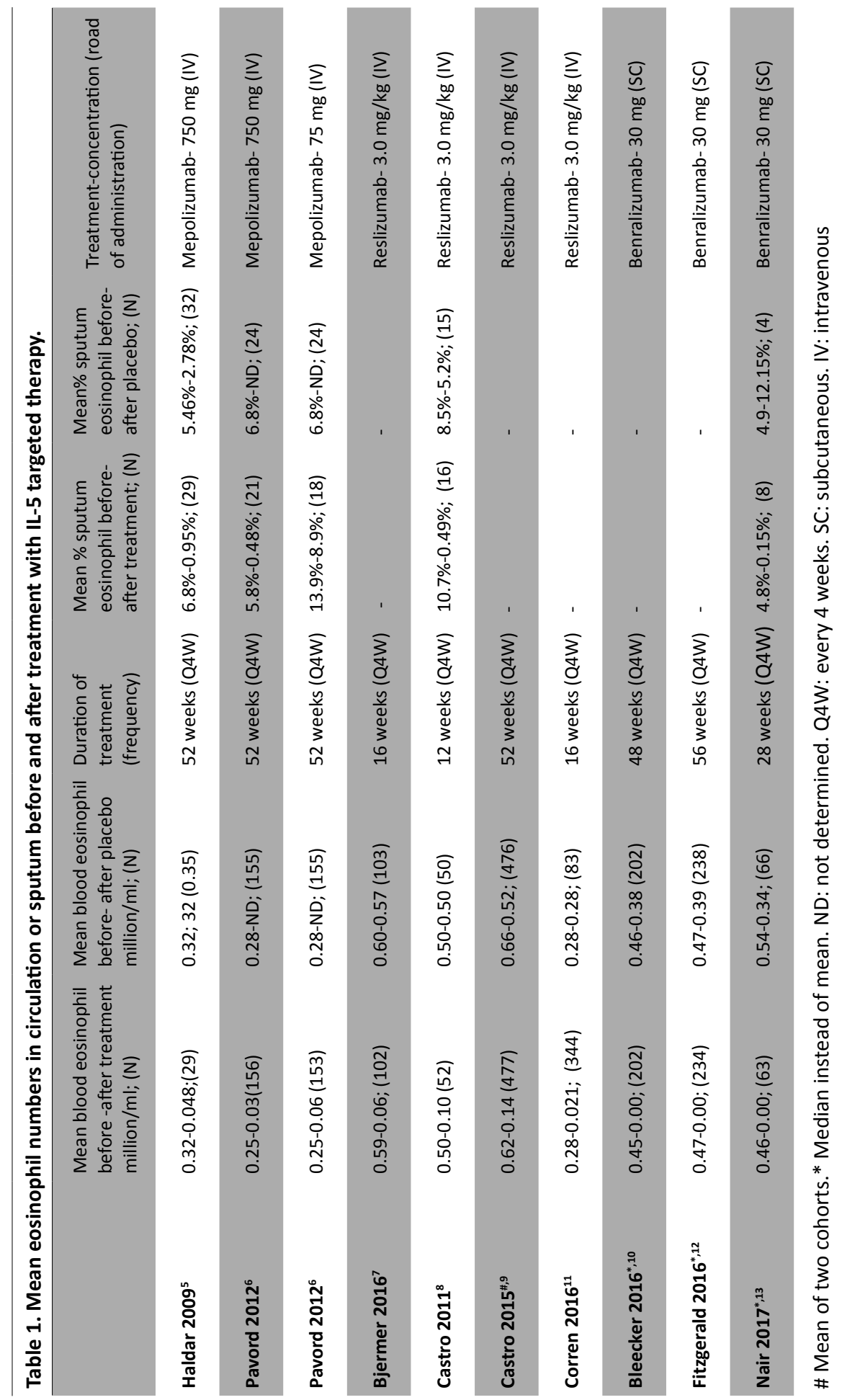


Sehmi et al. showed an increase of blood levels of eosinophil lineage committed progenitors compared to basal levels in patients who received 6 months of $100 \mathrm{mg}$ mepolizumab by subcutaneous injection and not in patients who received placebo. ${ }^{73}$ This finding contradicts the fact that IL-5 can upregulate its own receptor on CD34+ cells, since a reduction rather than in increase of EoPs would be expected. ${ }^{73,76}$ Another finding of the same study was that the number of EoPs and mature eosinophils in sputum was not attenuated in these patients with severe asthma. The authors hypothesized that this might be due to insufficient bioavailability within the tissue of $100 \mathrm{mg}$ mepolizumab opposed to $750 \mathrm{mg}$. Indeed, in a comparable cohort of patients with severe asthma and prednisolone dependence, the same investigators showed that the number of early progenitors (EoPs) in blood wasn't attenuated by a low dose of mepolizumab in contrast to a weight adjusted relatively high dose of intravenous reslizumab. ${ }^{14}$ The authors speculated that if also mepolizumab would have been administered high enough (weight adjusted) the number of EoPs would in fact be diminished with a consequent attenuation of sputum eosinophils. This hypothesis is, however, contradicted by the finding of an earlier study in which unaltered levels of EoPs in blood and bone marrow were found after treatment with $750 \mathrm{mg}$ Mepolizumab. ${ }^{74}$ In sputum the amount of CD34+/IL-5R $\alpha+$ cells was also attenuated after treatment with $750 \mathrm{mg}$ mepolizumab, but this was also seen with placebo. ${ }^{74}$

In contrast to the findings obtained with anti-IL-5 antibodies, a complete depletion of eosinophils and their early progenitors in the bone marrow was seen in a very small cohort of 4 patient with asthma that were treated with a single dose of 1 $\mathrm{mg} / \mathrm{kg}$ benralizumab (anti-IL-5R $\alpha$ ) via an intravenous injection. ${ }^{62}$ It is possible that this difference in the amount of bone marrow eosinophils and late progenitors in comparison with mepolizumab is caused by antibody dependent cell mediated cytotoxicity. ${ }^{24}$ Similarly EoPs are also strongly attenuated in blood and sputum after treatment with benralizumab. ${ }^{61}$

\section{Effect of anti-IL5(receptor) on cellular priming and activation}

IL-5 targeted therapy also affects the release of eosinophil basic proteins in these compartments. For instance, it has been described that eosinophil cationic protein (ECP) is found in enhanced levels in serum of asthma patients. ${ }^{77}$ Also increased concentrations of major basic protein $(\mathrm{MBP}){ }^{78}$ eosinophil cationic protein $(\mathrm{ECP})^{79}$ and eosinophilic peroxidase (EPO) were found in tissue in this patient group. ${ }^{70,77}$ These cytotoxic proteins can affect extracellular matrix (ECM)proteins in the reticular basement membrane (EMB). ${ }^{80}$ In addition, they can modulate TGF- $\beta 1$ expression by airway eosinophils. The concentrations of these mediators are blunted in BAL-fluid of patients with atopic asthma after treatment with mepolizumab. ${ }^{80}$ This suggests that anti-IL-5 also reduces the degree of activation of eosinophils in the tissue. This hypothesis is supported by the finding that treatment with anti-IL-5 leads to inhibition of the activation of eosinophils by anti-IL-5 in a segmental antigen challenge model. ${ }^{81}$ In this study the intermediate upregulation of $\beta_{2}$-integrin and the upregulation of P-selectin glycoprotein ligand-1 (PSGL-1) on circulating eosinophils was decreased after mepolizumab. ${ }^{81}$ Eosinophils of patients treated with mepolizumab have also showed a reduced eotaxin-induced shape change ex vivo. ${ }^{82}$

Treatment with anti-IL5R $\alpha$ antibodies (benralizumab) also caused a decreased 
concentration of ECP and eosinophil-derived neurotoxin (EDN) in serum, meaning that despite $A D C C$ no harmful proteins are released in blood. ${ }^{83}$

All of the above suggest that anti-IL-5(R $\alpha)$ not only attenuates eosinophil numbers but also reduces priming and/or activation of eosinophils and can therefore diminish inflammation and remodeling as well.

Despite these findings the situation with priming and activation in vivo is more complex than these in vitro data suggest. This complexity is illustrated by the finding that the expression of activation associated markers on eosinophils are not all pointing at complete suppression of eosinophil priming by IL-5(R $\alpha)$ targeted therapy. This is particularly shown by the expression of $\beta_{1}$-integrin in their active configuration in blood eosinophils, which was not altered by treatment with mepolizumab. ${ }^{81}$

Also the amount of major basic protein (MBP) in tissue was not found to drop with reduction of eosinophils. ${ }^{64} \mathrm{~A}$ possible explanation could be that the remaining eosinophils are still able to produce sufficient amounts of MBP or there is a different source of MBP-1. ${ }^{84}$ Alternative tissue sources of MBP other than placenta are poorly defined although mRNA of the MBP gene (PRG1) has been found in multiple tissues. ${ }^{85}$ Similarly unexpected was the finding that the rise of bronchoalveolar eosinophils after an allergen challenge was strongly reduced after administration of $750 \mathrm{mg}$ of mepolizumab intravenously whereas the remaining eosinophils still showed an IL-5 signature (enhanced expression of CD23, CD44, and CD69)..$^{70}$

\section{Effect of IL-5(R $\alpha)$ targeted therapy on IL-5 levels in blood and tissue}

Treatment with anti-IL-5 (mepolizumab or reslizumab) eventually results in an increase of plasma levels of IL-5 (bound to Mab?) in patients with asthma, hypereosinophilic syndrome and eosinophilic gastroenteritis even though there might be a slight decrease just after the start of treatment. ${ }^{82,86}$ This increase was also partially found in sputum of patients with asthma treated with mepolizumab but was surprisingly not seen in patients treated with reslizumab. ${ }^{14}$ The authors of the latter article concluded that this difference was probably due to a low local concentration of mepolizumab (and not of reslizumab) which consequently would have resulted in the formation of immune complexes because the target antigen would still be in excess. These presumably long lived immune complexes, would then be a stable source of IL-5. Although this may be a plausible hypothesis, direct proof is lacking. An increase of the plasma level of IL-5 was also seen with high doses of mepolizumab (750 mg) or an intermediate dose with reslizumab $(1 \mathrm{mg} / \mathrm{kg})$ in a small study. ${ }^{82,86}$ These data do not rule out the formation of immune complexes, but alternative hypotheses such as the existence of a feedback/feedforward mechanism are equally plausible. Indeed after treatment with mepolizumab there was a suggestion that both IL-5 producing CD4 and $\mathrm{CD}^{+}$cells increased in peripheral blood. ${ }^{82}$ This result has not been reproduced in another smaller study with reslizumab. ${ }^{86}$ It is, therefore, unfortunate that in the study of Mukherjee et al. no plasma levels of IL-5 were presented. ${ }^{14}$ An argument against a dominant role of immune complexes in modulating IL-5 levels in vivo came from data of a study on treating patients with benralizumab. Also in these patients treatment with this antibody independently of the dose $(25-200 \mathrm{mg})$ resulted in a clear increase of serum level of IL-5 while treating a similar cohort of patients with placebo did not. ${ }^{83}$ The increase in serum/plasma IL-5 regardless of its cause during treatment with 
anti-IL-5(R $\alpha)$ might have clinical consequences. For, there's a possibility that after termination of therapy a rebound eosinophilia may arise. This has been suggested before for patients with hyper eosinophilic syndrome, eosinophilic esophagitis and nasal polyps receiving a single dose of reslizumab (varying dose of $1-3 \mathrm{mg} / \mathrm{kg}$ ). ${ }^{53}$, 86-88 Remarkably this rebound eosinophilia was absent in asthma patients treated with a higher single dose of reslizumab: $1 \mathrm{mg} / \mathrm{kg}$ instead of $0.3 \mathrm{mg} / \mathrm{kg} .{ }^{88}$ Moreover in two studies in which patients were treated with multiple doses of mepolizumab at a higher dose of $10 \mathrm{mg} / \mathrm{kg}$ or $750 \mathrm{mg}$ a clear rebound phenomenon was also absent. ${ }^{82,89}$ Apparently, multiple pathways are in control of eosinophilia. Interesting in this regard is the finding that treatment with GM-CSF induces eosinophilia but only transiently. ${ }^{90}$ Nonetheless, these data implicate that discontinuation of the anti-IL-5 therapy should always be well monitored, since in some patients aggravation of symptoms have been described, particularly when steroids were tapered before cessation of the IL-5(R $\alpha)$ targeted therapy. ${ }^{82,89}$

\section{Before mepolizumab/ reslizumab}

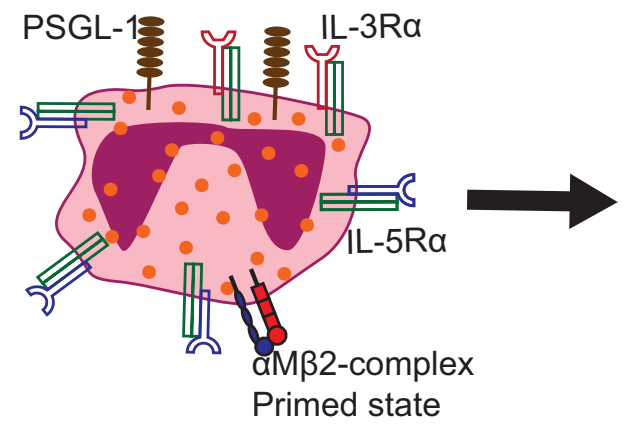

\section{After mepolizumab/ reslizumab}

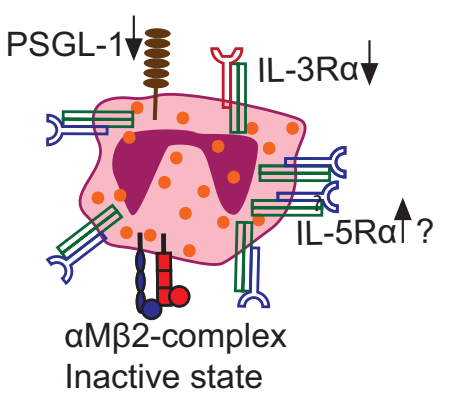

Figure 1.

Treatment with anti-IL -5 results in a decrease in IL -3R $\alpha$ expression on eosinophils in blood, while the expression of IL -5R $\alpha$ might increase. Besides eosinophils change from a primed state to a more inactive state, which is characterized by a reduced eotaxin-induced shape change and smaller size of eosinophils; downregulation of PSGL -1 and a change of configuration of the $(\alpha M) \beta 2$-complex.

\section{Effect of IL-5(R $\alpha)$ targeted therapy on IL-5R $\alpha$ and IL-3R $\alpha$ expression levels on eosinophils}

A next level of complexity in IL-5(R $\alpha)$ targeted therapy is the influence on the expression of cytokine receptors on eosinophils in vivo. Unfortunately, the effect of IL- 5 targeted therapy on IL-5R $\alpha$ expression on eosinophils hasn't been sufficiently established. The only data available imply that anti-IL-5 therapy resulted in a small reduction of the IL-5R $\alpha$ on eosinophils but the results were not convincing. ${ }^{82}$ This study of Stein et al. evaluated patients with different forms of eosinophilic inflammation that were treated with a high dose of mepolizumab. They found an $18 \%$ reduction of IL-5R $\alpha$-expression on blood eosinophils together with an increase rather than a decrease of plasma IL$5 .^{82}$ However, in two other placebo controlled studies performed in different cohorts this effect of anti-IL-5 on the expression of IL-5R was absent. ${ }^{77,91} \mathrm{~A}$ last relevant study 
was performed by Kelly et al. showing the same but not statistically significant trend of an increase of IL-5R $\alpha$ on eosinophils in asthma patients that were treated with a single dose of $750 \mathrm{mg}$ of mepolizumab after an antigen challenge. ${ }^{70} \mathrm{It}$ is not completely clear why IL-5R $\alpha$ expression on eosinophils would increase after anti-IL-5, but an autoregulatory pathway could be a possible explanation. Another explanation could be that IL-5 induced receptor shedding is naturally inhibited when less IL-5 is available, but since there's an increase of IL-5 after IL-5 inhibition this explanation is less likely. Surprisingly and still not fully understood in the study of Kelly et al was the downregulation of IL-3R $\alpha$ expression on blood eosinophils in asthma patients after treatment with mepolizumab. ${ }^{70}$ The authors speculated that this change might be induced by mepolizumab during eosinophilopoiesis by yet poorly understood mechanism(s). It might also be possible that IL-5 neutralization could have led to selective homing of IL-3R $\alpha^{\text {high }}$ cells into the tissue. ${ }^{70}$ Supporting this hypothesis was the finding that IL-3R $\alpha$ expression on BAL eosinophils after the antigen challenge was not attenuated. Alternatively, one might argue that the low expression was caused by in situ eosinophilopoiesis from mobilized (early) progenitors under the influence of IL-3 and/or GM-CSF instead of IL-5 leading to cells with specific expression profiles. This hypothesis is supported by a recent study ${ }^{63}$ potentially showing the existence of two distinct eosinophil subsets also in humans. This study of Mesnil et al. found difference in IL-3R $\alpha$ expression between lung parenchymal and peribronchial eosinophils of healthy human subjects and asthmatic patients. ${ }^{63} \mathrm{It}$ 's therefore tempting to speculate that these resident eosinophils are more dependent on IL-3 instead of IL-5. This might explain why tissue eosinophilia persists in patients treated with IL-5(R $\alpha)$ targeted therapy despite low levels of eosinophils in peripheral blood. ${ }^{69}$

\section{Conclusion}

\section{How to link the clinical action of IL-5(R $\alpha)$ targeted therapy with the cell biology of IL-5?}

Mechanisms of anti-IL-5 treatment are diverse and not fully understood (see graphical abstract and figure 1).

It's tempting to speculate why IL-5 targeted therapy is only beneficial for a part of the disease spectrum in patients with eosinophilic driven diseases. Firstly, eosinophils seem, especially in the tissue, only partially dependent on IL-5. It is still unclear whether these cells are the resident cells ${ }^{63}$ or that redundancy with IL-3 and GM-CSF is present in the system. Several important issues need to be addressed before robust conclusions can be drawn. These include: 1 . demonstration of the relative importance of local eosinophilopoiesis in affected tissues outside the bone marrow; 2 . direct identification of IL-5/anti-IL-5 immune complexes during anti-IL-5 therapy and 3. local survival of eosinophils due to other cytokines than IL-5 such as IL-3 or GM-CSF. It is also important to emphasize that inhibiting IL-5 doesn't lead to complete disarmament of eosinophils which means that remaining eosinophils are still able to release toxins that can cause tissue damage. Lastly, it's possible that eosinophil dominated inflammation in the tissue is not exclusively mediated by eosinophils. This latter observation is illustrated for eosinophilic asthma where patients might still experience symptoms and exacerbations because of neutrophils. ${ }^{92}$ Also a (near to) complete depletion of 
eosinophils in patients with eosinophilic asthma receiving benralizumab doesn't clearly lead to a better improvement in the reduction of asthma exacerbations compared to the treatment with anti-IL5. ${ }^{1}$ Interestingly, patients with severe "eosinophilic" asthma that are treated with dupilumab (anti-IL-4 receptor $\alpha$-subunit shared between IL-4 and IL-13 receptors ) show a comparable or maybe an even better clinical improvement while the number of eosinophils in circulation and in sputum remains unchanged. ${ }^{93}$ This indicates that even within the spectrum of Th2-driven diseases the pathogenesis of inflammation leading to tissue damage is complex. It is, therefore, not particularly surprising that single mediator antagonists only affect part of the disease spectrum in patients with complex immune mediated diseases. 


\section{References}

1. Farne HA, Wilson A, Powell C, Bax L, Milan SJ. Anti-IL5 therapies for asthma. Cochrane. Database. Syst. Rev 2017;9:CD010834

2. Rivero A, Liang J. Anti-IgE and Anti-IL5 Biologic Therapy in the Treatment of Nasal Polyposis: A Systematic Review and Meta-analysis. Ann. Otol. Rhinol. Laryngol 2017;126(11):739-747.

3. Wechsler ME, Akuthota P, Jayne D, Khoury P, Klion A, Langford CA, et al. Mepolizumab or Placebo for Eosinophilic Granulomatosis with Polyangiitis. N. Engl. J. Med 2017;376(20):19211932.

4. Rothenberg ME, Klion AD, Roufosse FE, Kahn JE, Weller PF, Simon HU, et al. Treatment of patients with the hypereosinophilic syndrome with mepolizumab. N. Engl. J. Med 2008;358(12):1215-1228.

5. Haldar P, Brightling CE, Hargadon B, Gupta S, Monteiro W, Sousa A, et al. Mepolizumab and exacerbations of refractory eosinophilic asthma. N. Engl. J. Med 2009;360(10):973-984.

6. Pavord ID, Korn S, Howarth P, Bleecker ER, Buhl R, Keene ON, et al. Mepolizumab for severe eosinophilic asthma (DREAM): a multicentre, double-blind, placebo-controlled trial. Lancet 2012;380(9842):651-659.

7. Bjermer L, Lemiere C, Maspero J, Weiss S, Zangrilli J, Germinaro M. Reslizumab for Inadequately Controlled Asthma With Elevated Blood Eosinophil Levels: A Randomized Phase 3 Study. Chest 2016;150(4):789-798.

8. Castro M, Mathur S, Hargreave F, Boulet LP, Xie F, Young J, et al. Reslizumab for poorly controlled, eosinophilic asthma: a randomized, placebo-controlled study. Am. J. Respir. Crit Care Med 2011;184(10):1125-1132.

9. Castro M, Zangrilli J, Wechsler ME, Bateman ED, Brusselle GG, Bardin P, et al. Reslizumab for inadequately controlled asthma with elevated blood eosinophil counts: results from two multicentre, parallel, double-blind, randomised, placebo-controlled, phase 3 trials. Lancet Respir. Med 2015;3(5):355-366.

10. Bleecker ER, FitzGerald JM, Chanez P, Papi A, Weinstein SF, Barker P, et al. Efficacy and safety of benralizumab for patients with severe asthma uncontrolled with high-dosage inhaled corticosteroids and long-acting beta2-agonists (SIROCCO): a randomised, multicentre, placebo-controlled phase 3 trial. Lancet 2016;388(10056):2115-2127.

11. Corren J, Weinstein S, Janka L, Zangrilli J, Garin M. Phase 3 Study of Reslizumab in Patients With Poorly Controlled Asthma: Effects Across a Broad Range of Eosinophil Counts. Chest 2016;150(4):799-810.

12. FitzGerald JM, Bleecker ER, Nair P, Korn S, Ohta K, Lommatzsch M, et al. Benralizumab, an anti-interleukin-5 receptor alpha monoclonal antibody, as add-on treatment for patients with severe, uncontrolled, eosinophilic asthma (CALIMA): a randomised, double-blind, placebo-controlled phase 3 trial. Lancet 2016;388(10056):2128-2141.

13. Nair P, Wenzel S, Rabe KF, Bourdin A, Lugogo NL, Kuna P, et al. Oral Glucocorticoid-Sparing Effect of Benralizumab in Severe Asthma. N. Engl. J. Med 2017;376(25):2448-2458.

14. Mukherjee M, Aleman PF, Kjarsgaard M, Salter B, Nair G, LaVigne N, et al. Weight-adjusted Intravenous Reslizumab in Severe Asthma with Inadequate Response to Fixed-dose Subcutaneous Mepolizumab. Am. J. Respir. Crit Care Med 2017.

15. Mukherjee M, Lim HF, Thomas S, Miller D, Kjarsgaard M, Tan B, et al. Airway autoimmune responses in severe eosinophilic asthma following low-dose Mepolizumab therapy. Allergy Asthma Clin. Immunol 2017;13:2. 
16. Nussbaum JC, Van Dyken SJ, von MJ, Cheng LE, Mohapatra A, Molofsky AB, et al. Type 2 innate lymphoid cells control eosinophil homeostasis. Nature 2013;502(7470):245-248.

17. Broughton SE, Nero TL, Dhagat U, Kan WL, Hercus TR, Tvorogov D, et al. The betac receptor family - Structural insights and their functional implications. Cytokine 2015;74(2):247-258.

18. Adachi T, Alam R. The mechanism of IL-5 signal transduction. Am. J. Physiol 1998;275(3 Pt 1):C623-C633.

19. Martinez-Moczygemba M, Huston DP. Biology of common beta receptor-signaling cytokines: IL-3, IL-5, and GM-CSF. J. Allergy Clin. Immunol 2003;112(4):653-665.

20. Coffer PJ, Koenderman L. Granulocyte signal transduction and priming: cause without effect? Immunol. Lett 1997;57(1-3):27-31.

21. McKinnon M, Page $K$, Uings IJ, Banks M, Fattah D, Proudfoot AE, et al. An interleukin 5 mutant distinguishes between two functional responses in human eosinophils. J. Exp. Med 1997;186(1):121-129.

22. Hercus TR, Bagley CJ, Cambareri B, Dottore M, Woodcock JM, Vadas MA, et al. Specific human granulocyte-macrophage colony-stimulating factor antagonists. Proc. Natl. Acad. Sci. U. S. A 1994;91(13):5838-5842.

23. Takatsu K. Interleukin-5 and IL-5 receptor in health and diseases. Proc. Jpn. Acad. Ser. B Phys. Biol. Sci 2011;87(8):463-485.

24. Kolbeck R, Kozhich A, Koike M, Peng L, Andersson CK, Damschroder MM, et al. MEDI-563, a humanized anti-IL-5 receptor alpha mAb with enhanced antibody-dependent cell-mediated cytotoxicity function. J. Allergy Clin. Immunol 2010;125(6):1344-1353.

25. Mori Y, Iwasaki H, Kohno K, Yoshimoto G, Kikushige Y, Okeda A, et al. Identification of the human eosinophil lineage-committed progenitor: revision of phenotypic definition of the human common myeloid progenitor. J. Exp. Med 2009;206(1):183-193.

26. Gregory B, Kirchem A, Phipps S, Gevaert P, Pridgeon C, Rankin SM, et al. Differential regulation of human eosinophil IL-3, IL-5, and GM-CSF receptor alpha-chain expression by cytokines: IL-3, IL-5, and GM-CSF down-regulate IL-5 receptor alpha expression with loss of IL-5 responsiveness, but up-regulate IL-3 receptor alpha expression. J. Immunol 2003;170(11):5359-5366.

27. Liu LY, Sedgwick JB, Bates ME, Vrtis RF, Gern JE, Kita H, et al. Decreased expression of membrane IL-5 receptor alpha on human eosinophils: I. Loss of membrane IL-5 receptor alpha on airway eosinophils and increased soluble IL-5 receptor alpha in the airway after allergen challenge. J. Immunol 2002;169(11):6452-6458.

28. Liu LY, Sedgwick JB, Bates ME, Vrtis RF, Gern JE, Kita H, et al. Decreased expression of membrane IL-5 receptor alpha on human eosinophils: II. IL-5 down-modulates its receptor via a proteinase-mediated process. J. Immunol 2002;169(11):6459-6466.

29. Wright AKA, Weston C, Rana BMJ, Brightling CE, Cousins DJ. Human group 2 innate lymphoid cells do not express the IL-5 receptor. J Allergy Clin Immunol 2017;140(5):1430-1433.e1434.

30. Smith SG, Chen R, Kjarsgaard M, Huang C, Oliveria JP, O'Byrne PM, et al. Increased numbers of activated group 2 innate lymphoid cells in the airways of patients with severe asthma and persistent airway eosinophilia. J Allergy Clin Immunol 2016;137(1):75-86.e78.

31. Sanderson CJ, Warren DJ, Strath M. Identification of a lymphokine that stimulates eosinophil differentiation in vitro. Its relationship to interleukin 3 , and functional properties of eosinophils produced in cultures. J. Exp. Med 1985;162(1):60-74.

32. Clutterbuck EJ, Hirst EM, Sanderson CJ. Human interleukin-5 (IL-5) regulates the production of eosinophils in human bone marrow cultures: comparison and interaction with IL-1, IL-3, IL-6, and GMCSF. Blood 1989;73(6):1504-1512. 
33. Denburg JA, Silver JE, Abrams JS. Interleukin-5 is a human basophilopoietin: induction of histamine content and basophilic differentiation of HL-60 cells and of peripheral blood basophil-eosinophil progenitors. Blood 1991;77(7):1462-1468.

34. Sanderson CJ. Interleukin-5, eosinophils, and disease. Blood 1992;79(12):3101-3109.

35. Yamaguchi Y, Suda T, Ohta S, Tominaga K, Miura Y, Kasahara T. Analysis of the survival of mature human eosinophils: interleukin-5 prevents apoptosis in mature human eosinophils. Blood 1991;78(10):2542-2547.

36. Horie $S$, Okubo $Y$, Hossain $M$, Sato $E$, Nomura $H$, Koyama $S$, et al. Interleukin-13 but not interleukin-4 prolongs eosinophil survival and induces eosinophil chemotaxis. Intern. Med 1997;36(3):179-185.

37. Owen WF, Jr., Rothenberg ME, Silberstein DS, Gasson JC, Stevens RL, Austen KF, et al. Regulation of human eosinophil viability, density, and function by granulocyte/macrophage colony-stimulating factor in the presence of 3T3 fibroblasts. J. Exp. Med 1987;166(1):129 141.

38. Kankaanranta H, Moilanen E, Zhang X. Pharmacological regulation of human eosinophil apoptosis. Curr. Drug Targets. Inflamm. Allergy 2005;4(4):433-445.

39. Duffin R, Leitch AE, Fox S, Haslett C, Rossi AG. Targeting granulocyte apoptosis: mechanisms, models, and therapies. Immunol. Rev 2010;236:28-40.

40. Farahi N, Singh NR, Heard S, Loutsios C, Summers C, Solanki CK, et al. Use of 111-Indiumlabeled autologous eosinophils to establish the in vivo kinetics of human eosinophils in healthy subjects. Blood 2012;120(19):4068-4071.

41. Steinbach KH, Schick P, Trepel F, Raffler H, Dohrmann J, Heilgeist G, et al. Estimation of kinetic parameters of neutrophilic, eosinophilic, and basophilic granulocytes in human blood. Blut 1979;39(1):27-38.

42. Parwaresch MR, Walle AJ, Arndt D. The peripheral kinetics of human radiolabelled eosinophils. Virchows Arch. B Cell Pathol 1976(1):57-66.

43. Dale DC, Hubert RT, Fauci A. Eosinophil kinetics in the hypereosinophilic syndrome. J. Lab Clin. Med 1976;87(3):487-495.

44. Xu J, Jiang F, Nayeri F, Zetterstrom O. Apoptotic eosinophils in sputum from asthmatic patients correlate negatively with levels of IL-5 and eotaxin. Respir. Med 2007;101(7):14471454.

45. Kano G, Almanan M, Bochner BS, Zimmermann N. Mechanism of Siglec-8-mediated cell death in IL-5-activated eosinophils: role for reactive oxygen species-enhanced MEK/ERK activation. J Allergy Clin Immunol 2013;132(2):437-445.

46. Na HJ, Hudson SA, Bochner BS. IL-33 enhances Siglec-8 mediated apoptosis of human eosinophils. Cytokine 2012;57(1):169-174.

47. Menzella F, Lusuardi M, Galeone C, Taddei S, Facciolongo N, Zucchi L. Mepolizumab for severe refractory eosinophilic asthma: evidence to date and clinical potential. Ther. Adv. Chronic. Dis 2016;7(6):260-277.

48. Shields RL, Whether WR, Zioncheck K, O'Connell L, Fendly B, Presta LG, et al. Inhibition of allergic reactions with antibodies to IgE. Int. Arch. Allergy Immunol 1995;107(1-3):308-312.

49. Caruso M, Morjaria J, Emma R, Amaradio MD, Polosa R. Biologic agents for severe asthma patients: clinical perspectives and implications. Intern. Emerg. Med 2017.

50. Cockle SM, Stynes G, Gunsoy NB, Parks D, Alfonso-Cristancho R, Wex J, et al. Comparative effectiveness of mepolizumab and omalizumab in severe asthma: An indirect treatment comparison. Respir Med 2017;123:140-148. 
51. Morita $\mathrm{H}$, Moro $\mathrm{K}$, Koyasu S. Innate lymphoid cells in allergic and nonallergic inflammation. J Allergy Clin Immunol 2016;138(5):1253-1264.

52. Gevaert P, Van Bruaene N, Cattaert T, Van Steen K, Van Zele T, Acke F, et al. Mepolizumab, a humanized anti-IL-5 mAb, as a treatment option for severe nasal polyposis. J Allergy Clin Immunol 2011;128(5):989-995.e981-988.

53. Gevaert P, Lang-Loidolt D, Lackner A, Stammberger H, Staudinger H, Van ZT, et al. Nasal IL-5 levels determine the response to anti-IL-5 treatment in patients with nasal polyps. J. Allergy Clin. Immunol 2006;118(5):1133-1141.

54. Bachert C, Sousa AR, Lund VJ, Scadding GK, Gevaert P, Nasser S, et al. Reduced need for surgery in severe nasal polyposis with mepolizumab: Randomized trial. J Allergy Clin Immunol 2017;140(4):1024-1031.e1014.

55. Sawas T, Dhalla S, Sayyar M, Pasricha PJ, Hernaez R. Systematic review with meta-analysis: pharmacological interventions for eosinophilic oesophagitis. Aliment Pharmacol Ther 2015;41(9):797-806.

56. Oldhoff JM, Darsow U, Werfel T, Katzer K, Wulf A, Laifaoui J, et al. Anti-IL-5 recombinant humanized monoclonal antibody (mepolizumab) for the treatment of atopic dermatitis. Allergy 2005;60(5):693-696.

57. Kumar RK, Foster PS. Are mouse models of asthma appropriate for investigating the pathogenesis of airway hyper-responsiveness? Front Physiol 2012;3:312.

58. Lee JJ, Jacobsen EA, Ochkur SI, McGarry MP, Condjella RM, Doyle AD, et al. Human versus mouse eosinophils: "that which we call an eosinophil, by any other name would stain as red". J. Allergy Clin. Immunol 2012;130(3):572-584.

59. Kobayashi T, lijima K, Kita H. Marked airway eosinophilia prevents development of airway hyper-responsiveness during an allergic response in IL-5 transgenic mice. J. Immunol 2003;170(11):5756-5763.

60. Pouliquen IJ, Kornmann O, Barton SV, Price JA, Ortega HG. Characterization of the relationship between dose and blood eosinophil response following subcutaneous administration of mepolizumab. Int. J. Clin. Pharmacol. Ther 2015;53(12):1015-1027.

61. Sehmi R, Lim HF, Mukherjee M, Huang C, Radford K, Newbold P, et al. Benralizumab attenuates airway eosinophilia in prednisone-dependent asthma. J Allergy Clin Immunol 2018.

62. Laviolette M, Gossage DL, Gauvreau G, Leigh R, Olivenstein R, Katial R, et al. Effects of benralizumab on airway eosinophils in asthmatic patients with sputum eosinophilia. $J$. Allergy Clin. Immunol 2013;132(5):1086-1096.

63. Mesnil C, Raulier S, Paulissen G, Xiao X, Birrell MA, Pirottin D, et al. Lung-resident eosinophils represent a distinct regulatory eosinophil subset. J. Clin. Invest 2016;126(9):3279-3295.

64. Flood-Page PT, Menzies-Gow AN, Kay AB, Robinson DS. Eosinophil's role remains uncertain as anti-interleukin-5 only partially depletes numbers in asthmatic airway. Am. J. Respir. Crit Care Med 2003;167(2):199-204.

65. Kampfer SS, Odermatt A, Dahinden CA, Fux M. Late IL-3-induced phenotypic and functional alterations in human basophils require continuous IL-3 receptor signaling. J. Leukoc. Biol 2017;101(1):227-238.

66. Yamada T, Sun Q, Zeibecoglou K, Bungre J, North J, Kay AB, et al. IL-3, IL-5, granulocytemacrophage colony-stimulating factor receptor alpha-subunit, and common beta-subunit expression by peripheral leukocytes and blood dendritic cells. J. Allergy Clin. Immunol 1998;101(5):677-682. 
67. Otani IM, Anilkumar AA, Newbury RO, Bhagat M, Beppu LY, Dohil R, et al. Anti-IL-5 therapy reduces mast cell and IL-9 cell numbers in pediatric patients with eosinophilic esophagitis. J. Allergy Clin. Immunol 2013;131(6):1576-1582.

68. Dahl C, Hoffmann HJ, Saito H, Schiotz PO. Human mast cells express receptors for IL-3, IL-5 and GM-CSF; a partial map of receptors on human mast cells cultured in vitro. Allergy 2004;59(10):1087-1096.

69. Conus S, Straumann A, Bettler E, Simon HU. Mepolizumab does not alter levels of eosinophils, T cells, and mast cells in the duodenal mucosa in eosinophilic esophagitis. J. Allergy Clin. Immunol 2010;126(1):175-177.

70. Kelly EA, Esnault S, Liu LY, Evans MD, Johansson MW, Mathur S, et al. Mepolizumab Attenuates Airway Eosinophil Numbers, but Not Their Functional Phenotype in Asthma. Am. J. Respir. Crit Care Med 2017.

71. Nussbaum JC, Van Dyken SJ, von Moltke J, Cheng LE, Mohapatra A, Molofsky AB, et al. Type 2 innate lymphoid cells control eosinophil homeostasis. Nature 2013;502(7470):245-248.

72. Gauvreau GM, Denburg JA. Hemopoietic progenitors: the role of eosinophil/basophil progenitors in allergic airway inflammation. Expert. Rev. Clin. Immunol 2005;1(1):87-101.

73. Sehmi R, Smith SG, Kjarsgaard M, Radford K, Boulet LP, Lemiere C, et al. Role of local eosinophilopoietic processes in the development of airway eosinophilia in prednisonedependent severe asthma. Clin. Exp. Allergy 2016;46(6):793-802.

74. Menzies-Gow A, Flood-Page P, Sehmi R, Burman J, Hamid Q, Robinson DS, et al. Anti-IL-5 (mepolizumab) therapy induces bone marrow eosinophil maturational arrest and decreases eosinophil progenitors in the bronchial mucosa of atopic asthmatics. J. Allergy Clin. Immunol 2003;111(4):714-719.

75. Leentjens J, Kox M, Koch RM, Preijers F, Joosten LA, van der Hoeven JG, et al. Reversal of immunoparalysis in humans in vivo: a double-blind, placebo-controlled, randomized pilot study. Am. J. Respir. Crit Care Med 2012;186(9):838-845.

76. Tavernier J, Van der Heyden J, Verhee A, Brusselle G, Van O, X, Vandekerckhove J, et al. Interleukin 5 regulates the isoform expression of its own receptor alpha-subunit. Blood 2000;95(5):1600-1607.

77. Buttner C, Lun A, Splettstoesser T, Kunkel G, Renz H. Monoclonal anti-interleukin-5 treatment suppresses eosinophil but not T-cell functions. Eur. Respir. J 2003;21(5):799-803.

78. Frigas E, Loegering DA, Solley GO, Farrow GM, Gleich GJ. Elevated levels of the eosinophil granule major basic protein in the sputum of patients with bronchial asthma. Mayo Clin. Proc 1981;56(6):345-353.

79. Virchow JC, Jr., Holscher U, Virchow C, Sr. Sputum ECP levels correlate with parameters of airflow obstruction. Am. Rev. Respir. Dis 1992;146(3):604-606.

80. Flood-Page P, Menzies-Gow A, Phipps S, Ying S, Wangoo A, Ludwig MS, et al. Anti-IL-5 treatment reduces deposition of ECM proteins in the bronchial subepithelial basement membrane of mild atopic asthmatics. J. Clin. Invest 2003;112(7):1029-1036.

81. Johansson MW, Gunderson KA, Kelly EA, Denlinger LC, Jarjour NN, Mosher DF. Anti-IL-5 attenuates activation and surface density of beta(2) -integrins on circulating eosinophils after segmental antigen challenge. Clin. Exp. Allergy 2013;43(3):292-303.

82. Stein ML, Villanueva JM, Buckmeier BK, Yamada Y, Filipovich AH, Assa'ad AH, et al. Anti-IL-5 (mepolizumab) therapy reduces eosinophil activation ex vivo and increases IL-5 and IL-5 receptor levels. J. Allergy Clin. Immunol 2008;121(6):1473-1483, 1483. 
83. Pham TH, Damera G, Newbold P, Ranade K. Reductions in eosinophil biomarkers by benralizumab in patients with asthma. Respir. Med 2016;111:21-29.

84. Acharya KR, Ackerman SJ. Eosinophil granule proteins: form and function. J. Biol. Chem 2014;289(25):17406-17415.

85. The human protein atlas. In: PRG2 gene; 2018.

86. Kim YJ, Prussin C, Martin B, Law MA, Haverty TP, Nutman TB, et al. Rebound eosinophilia after treatment of hypereosinophilic syndrome and eosinophilic gastroenteritis with monoclonal anti-IL-5 antibody SCH55700. J. Allergy Clin. Immunol 2004;114(6):1449-1455.

87. Klion AD, Law MA, Noel P, Kim YJ, Haverty TP, Nutman TB. Safety and efficacy of the monoclonal anti-interleukin-5 antibody $\mathrm{SCH} 55700$ in the treatment of patients with hypereosinophilic syndrome. Blood 2004;103(8):2939-2941.

88. Kips JC, O'Connor BJ, Langley SJ, Woodcock A, Kerstjens HA, Postma DS, et al. Effect of SCH55700, a humanized anti-human interleukin-5 antibody, in severe persistent asthma: a pilot study. Am. J. Respir. Crit Care Med 2003;167(12):1655-1659.

89. Haldar P, Brightling CE, Singapuri A, Hargadon B, Gupta S, Monteiro W, et al. Outcomes after cessation of mepolizumab therapy in severe eosinophilic asthma: a 12-month follow-up analysis. J. Allergy Clin. Immunol 2014;133(3):921-923.

90. Olver IN, Hercus T, Lopez A, Vadas M, Somogyi AA, Doyle I, et al. A phase I study of the GMCSF antagonist E21R. Cancer Chemother. Pharmacol 2002;50(3):171-178.

91. Conus S, Straumann A, Simon HU. Anti-IL-5 (mepolizumab) therapy does not alter IL-5 receptor alpha levels in patients with eosinophilic esophagitis. J. Allergy Clin. Immunol 2009;123(1):269-270.

92. Denlinger LC, Sorkness RL, Lee WM, Evans MD, Wolff MJ, Mathur SK, et al. Lower airway rhinovirus burden and the seasonal risk of asthma exacerbation. Am. J. Respir. Crit Care Med 2011;184(9):1007-1014.

93. Wenzel S, Castro M, Corren J, Maspero J, Wang L, Zhang B, et al. Dupilumab efficacy and safety in adults with uncontrolled persistent asthma despite use of medium-to-highdose inhaled corticosteroids plus a long-acting beta2 agonist: a randomised double-blind placebo-controlled pivotal phase 2 b dose-ranging trial. Lancet 2016;388(10039):31-4 


\title{
Chapter 7
}

\section{Characterization of the phenotype of human eosinophils and their progenitors in the bone marrow of healthy individuals}

\begin{abstract}
Marwan Hassani, ${ }^{1,2}$, Selma van Staveren, ${ }^{1,2,3}$, Erinke van Grinsven, ${ }^{1,2}$, Marije Bartels, ${ }^{4}$ Kiki Tesselaar, ${ }^{2}$, Guus Leijte, ${ }^{5}$ Matthijs Kox, ${ }^{5}$, Peter Pickkers ${ }^{5}$ Nienke Vrisekoop, ${ }^{1,2}$, Leo Koenderman, ${ }^{1,2}$
\end{abstract}

1. Department of Respiratory Medicine, University Medical Centre Utrecht, Utrecht, The Netherlands.

2. Laboratory of Translational Immunology, University Medical Centre Utrecht, Utrecht, The Netherlands. 3. TI-COAST, Science Park 904, 1098 HX Amsterdam, the Netherlands 4. Department of Pediatric Hematology, University Medical Centre Utrecht, Utrecht, The Netherlands. 5. Department of Intensive Care and Radboud Center for Infectious Diseases (RCI), Radboud University Medical Center, Nijmegen, The Netherlands 
Eosinophils are important innate effector cells in the protection of the host against invading multicellular parasites, but also play a deleterious role in allergic diseases. Previously, the earliest committed progenitor for eosinophils was identified to be $\mathrm{CD} 4^{+}$and IL-5R $\alpha^{+}$. However, the knowledge concerning unique membrane markers to identify the subsequent maturation stages of eosinophils has been very limited. In this study we have identified multiple membrane makers that distinguish these different middle-late maturation stages of eosinophils in the bone marrow (BM) of healthy human volunteers.

BM and peripheral blood were collected from 12 healthy volunteers. Eosinophilic promyelocytes, myelocytes, metamyelocytes and mature eosinophils were morphologically identified and FACS sorted according to their CD11b/CD62L expression. In addition, the expression of other antibodies against known membrane markers on eosinophils: LAIR1 (CD305), Alpha-4 (CD49d), CCR3 (CD193), FcgRIII (CD64), CR1 (CD35), CEACAM- 8 (CD66b), IL-5R $\alpha$ (CD125) and IL-3R $\alpha$ (CD123) was determined.

Classically, eosinophils are considered as essential innate effector cells in protection of the host against invading multicellular parasites (particularly helminths) by releasing a variety of preformed potent granular mediators and the production of toxic reactive oxygen species. ${ }^{1}$ The synergism between these two mechanisms sufficiently arms these cells to kill large pathogens, at least in vitro. ${ }^{2}$ At the same time, clonal proliferation of eosinophil precursors can lead to chronic eosinophilic leukemia, a disease accompanied by cardiovascular, respiratory and gastrointestinal symptoms. ${ }^{3}$

Despite the consensus regarding the importance of these cells in homeostasis and disease, remarkably little is known about their life cycle and maturation in the bone marrow (BM) in homeostasis ("health"). Albeit, it has been shown that IL-5 receptor expression in BM-derived CD34 ${ }^{+}$myeloblasts defines the characterization of the earliest committed eosinophil precursor. ${ }^{4,5}$ Nevertheless, the fate of these myeloblasts and the subsequent maturation of eosinophil progenitors in the BM has not been studied so far. In the present study, we have developed a method to identify the different middle-late maturation stages of eosinophils in the BM of healthy volunteers by flow cytometry analysis. This knowledge provides a first critical step in delineating mechanisms underlying normal eosinophil development and eosinophilic disorders. 
Table 1. P-values of the comparison of the different surface markers between all stages of eosinophil maturation.

\begin{tabular}{|c|c|c|c|c|c|c|c|c|c|c|c|c|c|c|}
\hline$\stackrel{d}{\stackrel{7}{0}}$ & ন্. & ठ̀ & o̊̀ & in & 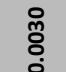 & ठํ. & ठั & $\begin{array}{l}\vec{I} \\
0\end{array}$ & $\begin{array}{l}\circ \\
\stackrel{1}{0}\end{array}$ & $\begin{array}{l}\text { ô } \\
\text { O. } \\
\text { o. }\end{array}$ & $\stackrel{o}{0}_{0}^{0}$ & 芩 & ס̊ & : \\
\hline$\underset{0}{\tilde{O}}$ & ठ் & ठ் & 잉 & $\stackrel{\vec{n}}{\tilde{O}}$ & ôे & ఫे & $\stackrel{\infty}{\stackrel{\infty}{0}}$ & 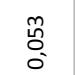 & $\begin{array}{l}\infty \\
\stackrel{-}{0}\end{array}$ & 옹 & ठ̊̊̊ & $\begin{array}{l}\stackrel{o}{a} \\
\hat{o} \\
0\end{array}$ & : & $\stackrel{m}{0}_{0}^{0}$ \\
\hline 영 & 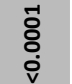 & ষ্் & $\begin{array}{l}\text { ô } \\
\text { Oे. } \\
\text { O. }\end{array}$ & 宫 & $\begin{array}{l}0 \\
\stackrel{0}{0} \\
0 \\
0\end{array}$ & $\begin{array}{l}\text { ț } \\
\text { : }\end{array}$ & $\underset{\text { Ñ }}{0}$ & & 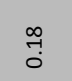 & 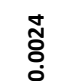 & 홍 & बें & 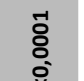 & ذ్: \\
\hline 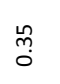 & $\begin{array}{l}\stackrel{0}{0} \\
\stackrel{0}{0}\end{array}$ & ठ̀̊ & $\begin{array}{l}\overrightarrow{0} \\
\text { ò } \\
\dot{v}\end{array}$ & mo & $\begin{array}{l}\infty \\
: \\
0 \\
0\end{array}$ & $\begin{array}{l}\text { مٌ } \\
\text { ơ }\end{array}$ & 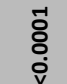 & $\begin{array}{l}\text { ồ } \\
\text { O̊. }\end{array}$ & 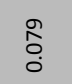 & م̊̊ & $\begin{array}{l}\text { वे } \\
\text { ᄋ̆ }\end{array}$ & $\stackrel{\substack{m \\
0}}{0}$ & 离 & : \\
\hline ర్రి & $\begin{array}{l}-1 \\
\text { ¿ } \\
\dot{0} \\
\mathrm{v}\end{array}$ & $\begin{array}{l}\text { no } \\
\text { o. }\end{array}$ & $\stackrel{\sim}{0}$ & 응 & $\begin{array}{l}\qquad 0 \\
0 \\
0\end{array}$ & 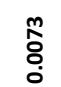 & 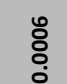 & & $\stackrel{\text { }}{\tilde{O}}$ & & ¿্் & \begin{tabular}{l}
0 \\
\multirow{2}{0}{} \\
0
\end{tabular} & $\underset{\tilde{\Xi}}{\tilde{\Xi}}$ & z \\
\hline 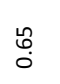 & ồ & ठ্் & $\begin{array}{l}\text { 일 } \\
\text { ơ }\end{array}$ & $\stackrel{m}{m}$ & 웅 & ठั & $\begin{array}{l}\text { \&̊․ } \\
\text { ס्र }\end{array}$ & $\begin{array}{l}\infty \\
\stackrel{\infty}{0} \\
0 \\
0\end{array}$ & $\overline{0}$ & $\stackrel{\widetilde{N}}{0}$ & ¿̈ & $\stackrel{\infty}{\sim}$ & : & ठั \\
\hline$\underset{0}{0}$ & ذั & $\stackrel{n}{0}$ & ڤ̆ & o & 蒿 & ণ্ণ & $\stackrel{\text { å }}{0}$ & $\begin{array}{l}0 \\
\infty \\
0 \\
0\end{array}$ & ֻे & ठัે & $\begin{array}{l}\mathscr{0} \\
:\end{array}$ & $\stackrel{m}{0}$ & $\begin{array}{l}\text { थू } \\
\text { o. }\end{array}$ & $\begin{array}{l}\text { N } \\
0 \\
0\end{array}$ \\
\hline $\begin{array}{l}\text { g. } \\
0 \\
0\end{array}$ & ปั & वे & $\begin{array}{l}\overrightarrow{0} \\
80 \\
\dot{0}\end{array}$ & $\begin{array}{l}\hat{o ̂} \\
\text { o. }\end{array}$ & ชั & वे & $\begin{array}{l}\overrightarrow{0} \\
\dot{0} \\
\dot{v}\end{array}$ & $\begin{array}{l}\overrightarrow{\tilde{z}} \\
\text { : }\end{array}$ & స్ & ồ & స̃ & 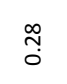 & : & ¿̊̀ \\
\hline ồ & ₹ี & $\underset{\text { ָ̦ }}{\text { N }}$ & 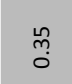 & 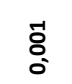 & ö & ठ் & ठํํ & के & ֻे & $\begin{array}{l}\stackrel{0}{0} \\
\stackrel{0}{0}\end{array}$ & ষ̊ & $\begin{array}{l}\stackrel{2}{0} \\
\hat{0}\end{array}$ & $\begin{array}{l}\text { ơ } \\
0 \\
0\end{array}$ & 今ิ \\
\hline $\begin{array}{l}0 \\
\stackrel{0}{0}\end{array}$ & 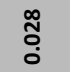 & $\begin{array}{l}0 \\
\stackrel{0}{0} \\
0\end{array}$ & 之 & $\begin{array}{c}\text { \& } \\
0 \\
0\end{array}$ & ন্ & 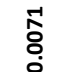 & 之 & 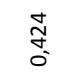 & $\underset{\text { ָ̃ }}{0}$ & 之 & 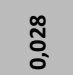 & 之 & ¿̈ & $\hat{z}$ \\
\hline$\underset{0}{\tilde{O}}$ & ర్త & $\begin{array}{l}0 \\
\text { ô } \\
0\end{array}$ & ठ̊ & oิ & 苂 & $\begin{array}{l}\text { 옴 } \\
\text { O. }\end{array}$ & ठ̊ & ö & :̊ & $\begin{array}{l}\stackrel{2}{0} \\
\stackrel{0}{0}\end{array}$ & $\begin{array}{l}\overrightarrow{0} \\
\text { o. }\end{array}$ & $\begin{array}{l}\text { \%े } \\
0 \\
0\end{array}$ & ठิ & ठ̀̊ \\
\hline 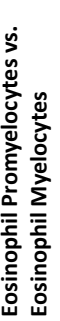 & 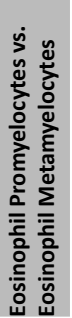 & 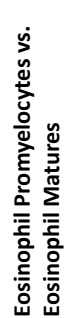 & 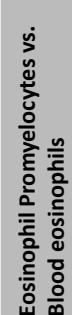 & 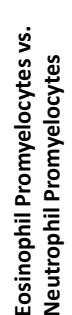 & 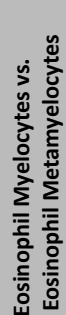 & 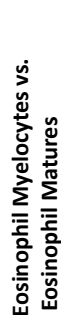 & 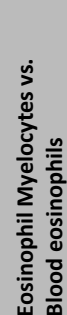 & 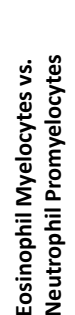 & 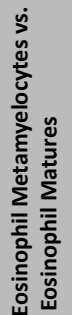 & 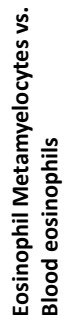 & 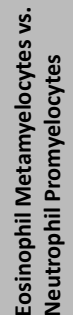 & 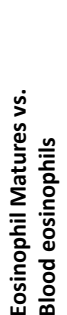 & 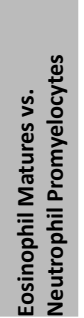 & 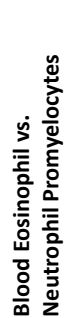 \\
\hline
\end{tabular}

All p-values were calculated using a Friedman's test followed by an uncorrected Dunn's test. 
Blood and bone marrow samples were collected from 12 healthy male volunteers. All individuals had no prior history of atopic disease. Bone marrow aspirates were obtained from the iliac crest and blood was obtained by venipuncture. Blood was collected in sodium heparin tubes (Vacuette ${ }^{\circledR}$ Greiner bio-one, Kremsmünster, Austria) and BM aspirates were collected in Falcon tubes, which were prefilled with isotonic sodium heparin (sodium heparin $150 \mathrm{El} / \mathrm{ml}: \mathrm{BM}=1: 3$ ). Subsequently, erythrocytes were lysed using an ice cold lysis buffer ( $150 \mathrm{mM} \mathrm{NH}_{4} \mathrm{Cl}, 10 \mathrm{mM} \mathrm{KHCO}_{3}$ and $0.1 \mathrm{mM} \mathrm{NA}_{2}$ EDTA). Hereafter, the remaining leukocytes were washed and resuspended in a staining buffer consisting of PBS supplemented with $0.32 \% \mathrm{w} / \mathrm{v}$ trisodium citrate (both prepared by the pharmacy of the University Medical Centre Utrecht) and $10 \% \mathrm{w} / \mathrm{v}$ human pasteurized plasma solution (Sanquin, Amsterdam, the Netherlands). One million cells at a concentration of 40 million cells per $\mathrm{mL}$ were stained with antibodies for 30 minutes on ice, and washed twice before analysis on the LSR-Fortessa flow cytometer (Becton Dickinson (BD), Mountain View CA, USA). The following antibodies were used for staining: from BD: IL-3R $\alpha$-PerCP-Cy5.5 (clone 7G3), IL-5R $\alpha$-BV421 (clone A14), CD62L-BV650 (clone DREG-56) and LAIR1-PE (clone DX26); from Biolegend (San Diego, CA, USA): CD64-APC (clone 10.1), CD193-BV510 (clone 5E8), Siglec-8-PE (clone 7C9), CD66b PerCP-Cy5.5(clone G10F5), CD16-BV785 (clone 3G8), CD49d-PE-Cy7 (clone 9F10) and CD35-FITC (clone E11) and from Beckman Coulter (Pasadena, CA, USA): CD11b-APC-AF750 (clone Bear1). Viability of the whole bone marrow sample was assessed separately by staining in PBS with the Fixable Violet Dead Cell Stain Kit (Life technologies, Thermo Fisher Scientific, Waltham, MA, USA). Different cell populations were sorted using an Aria III FACS sorter (BD, Mountain View, CA, USA) and stained with May-Grünwald-Giemsa. Cells were then microscopically scored on the basis of nuclear morphology and cytosolic/granule staining.

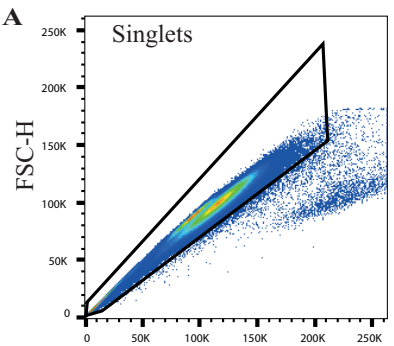

FSC-A

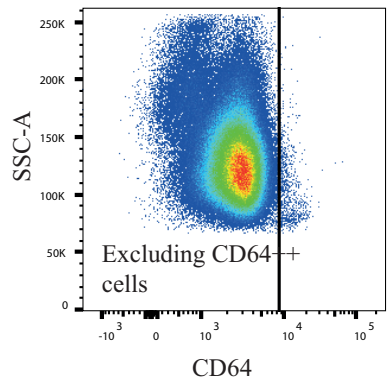

CD64

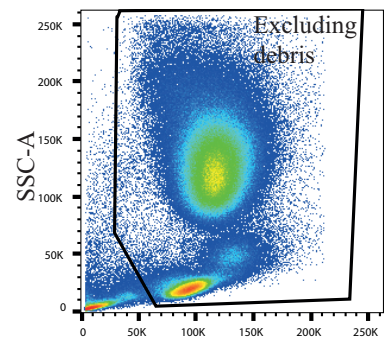

FSC-A

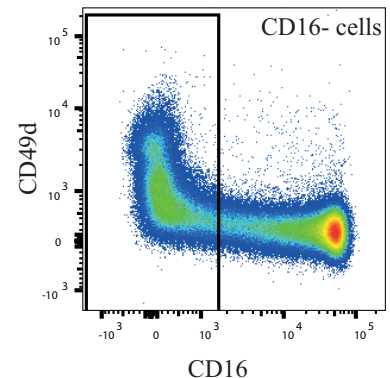

CD16

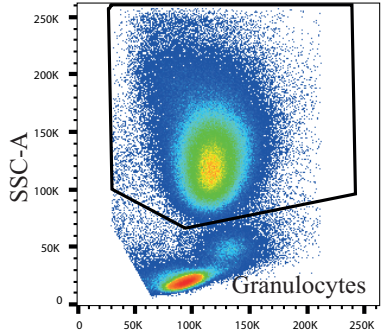

FSC-A

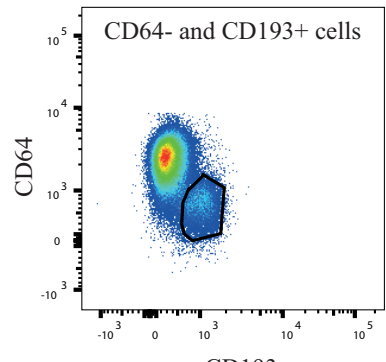

CD193 


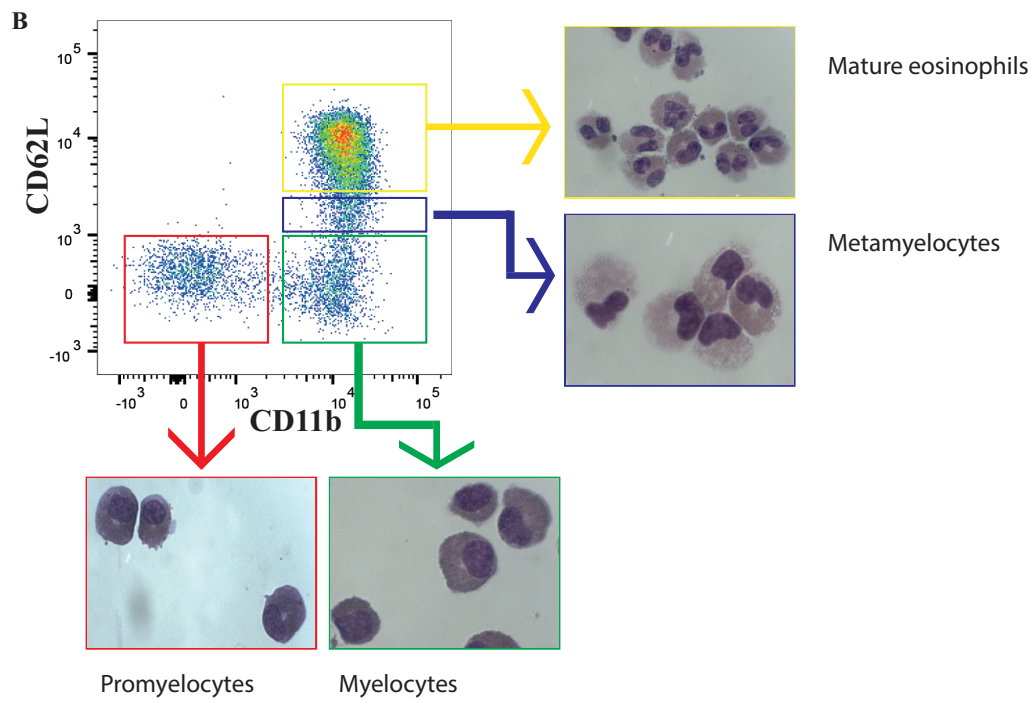

Figure 1. Gating strategy to identify eosinophils in blood and bone marrow.

A. Example of a gating strategy to identify all eosinophils and their progenitors in the bone marrow. Granulocytes in blood and BM were identified on the basis of FSC/SSC. After that monocytes were excluded using CD64. Subsequently eosinophils and their progenitors were identified as CD16, CD64 low and $\mathrm{CD}_{193}{ }^{+}$cells.

B. After gating all eosinophils and their progenitors, the different stages of eosinophil maturation can be identified using CD62L and CD11b. Representative cytospins of sorted cells after staining with MayGrünwald-Giemsa (Objective used: 100x, Axioskop 40, Light microscopy, Carl Zeiss, Goettingen, Germany) of the different stages are shown for each gate.

Characterization of the male volunteers was: age $23 \pm 1$ years (mean \pm SEM), weight $85 \pm 2 \mathrm{~kg}$, height $1.84 \pm 0.02 \mathrm{~m}$ and BMI $25.0 \pm 0.4 \mathrm{~kg} / \mathrm{m} 2$. The concentration of blood eosinophils was $0.14 \pm 0.091$ million cells $/ \mathrm{ml}$.

Bone marrow and blood eosinophils and their middle-late precursors were first identified based on FSC/SSC. Dead cells were excluded based on FSC and SSC. Next, cells with a high SSC were gated followed by exclusion of monocytes using CD64 expression. Subsequently eosinophils and their progenitors were identified as CD16, CD64 ${ }^{\text {low }}$ and $C D 193^{+}$cells (Figure 1A). We applied two markers, CD11b and CD62L to distinguish between immature and mature eosinophils. These two markers provided a similar expression pattern as is seen with CD16 and CD11b in neutrophils (Supplementary Figure 1). ${ }^{6}$ Analyzing both markers in a biplot revealed 4 different populations of cells in the eosinophil gate (Figure 1B). We applied FACS sorting and microscopic evaluation of cytospin preparations to isolate and morphologically assess these 4 different groups. Based on different nuclear morphology and cytosolic/granule staining as described before ${ }^{7}$, we observed that $\mathrm{CD}_{11} \mathrm{~b}^{-}$and $\mathrm{CD}^{\mathrm{C}} \mathrm{L}^{-}$ cells showed characteristics of eosinophilic promyelocytes, CD11 bright and CD62L' cells of eosinophilic myelocytes, CD11 bright and CD62L ${ }^{\text {dim }}$ cells of eosinophilic metamyelocytes and finally CD11 b ${ }^{\text {bright }}$ and CD62 $\mathrm{L}^{\text {bright }}$ cells resembled mature eosinophils (Figure $1 \mathrm{~B}$ and Supplementary Figure 1). A direct comparison of cytospins from sorted neutrophilic and 
eosinophilic precursors showed that eosinophilic precursors were characterized by the eosinophils-specific (dark) orange granules that were lacking in neutrophil precursors (Supplementary Figure 1). The purity of the different sorted populations was at least $80 \%$. Staining cells separately for viability showed a viability of at least $95 \%$ and non-viable cells were eventually gated out on the basis of FSC and SSC and not analyzed.

After validating our gating strategy, we then assessed putative changes in expression of eosinophil surface markers during maturation (Figure 2 and Table 1). Cell surface expression of the eotaxin receptor CCR3 (CD193) and siglec- 8 were both upregulated during maturation. The comparison of these markers with neutrophils and neutrophil progenitors, which do not express these proteins, ${ }^{8,9}$ indicated that the eosinophilic promyelocytes or myelocytes also expressed both proteins at low levels (Figure 2 and Supplementary Figure 2). We also gated eosinophils and their progenitors independently of CD193 by using only CD64 and CD49d, because the initial gating strategy involved CD193 (Supplementary Figure 1). This alternative method showed a similar CD11b and CD62L biplot for eosinophil progenitors demonstrated in Figure 1B. With this gating strategy we could still detect a higher expression of CD193 on eosinophilic promyelocytes compared to neutrophilic promyelocytes (Figure 2A). The expression of the $\alpha$ chain of VLA-4 (CD49d) and CEACAM-8 (CD66b) reached peak levels during the myelocyte stage and the expression of both proteins was maintained throughout maturation. (Figure $2 \mathrm{C}$ and $2 \mathrm{G}$ ). This is in marked contrast to neutrophils, which lose CD49d expression after the myelocyte stage..$^{10}$ The $\alpha$-chain of the IL-5 receptor (CD125) was expressed during all stages of maturation, but the expression was highest on mature eosinophils in the BM and circulation (Figure 2D). The expression of the $\alpha$ chain of the IL-3 receptor (CD123) was quite variable amongst the different maturation stages (Figure 2E). However, the overall expression was low limiting the interpretation of this finding.

Similarly to neutrophils, ${ }^{11}$ the expression of complement receptor 1 (CD35) was late during maturation of eosinophils and was only found on mature eosinophils (Figure 2F). LAIR1 (CD305) was expressed on all stages of eosinophil maturation, but its expression was highest during the promyelocyte and myelocyte stages. This expression pattern is comparable with neutrophils. ${ }^{12}$ Finally, the expression of Fcy receptor I (CD64) was only found on eosinophil promyelocytes albeit at a low level. This is in contrast to neutrophils where CD64 expression is maintained until the metamyelocyte stage. ${ }^{13}$

This is the first study characterizing different stages of eosinophil maturation in the human BM during homeostasis using the expression of a variety of membrane markers. Using this method we did not find clear indications for banded eosinophils as has been described for neutrophils. ${ }^{14} \mathrm{~A}$ limitation of this study, however, is the lack of other validation methods such as IL-5 responsiveness or differences in transcriptome of the different sorted populations. Nonetheless the distinct nuclear morphology and cytosol staining of the sorted populations in combination with a gradual increase in expression of several eosinophil markers on the different maturation stages will facilitate future studies focusing on the differentiation in eosinophilic diseases.

Concerning the eosinophil maturation process, we propose a model in which eosinophil progenitors are proliferating and maturing as being on a linear "conveyor belt". A comparable model was first described for neutrophils in 1964 by Cartwright et al. ${ }^{15}$ It assumes that cells gradually go through each phase: proliferation and maturation of promyelocytes and myelocytes in a mitotic pool that is followed by maturation of metamyelocytes and 
mature cells in a post-mitotic pool. This hypothesis is supported by the observations that various surface markers such as CD193, IL-5R $\alpha$ and Siglec- 8 are gradually upregulated upon maturation and reach highest levels in mature circulatory eosinophils.
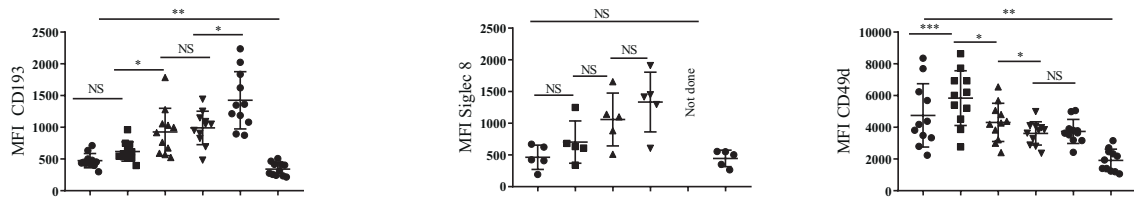

D

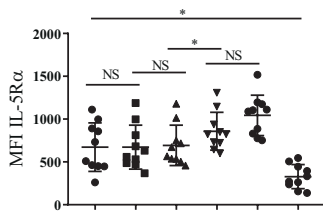

G

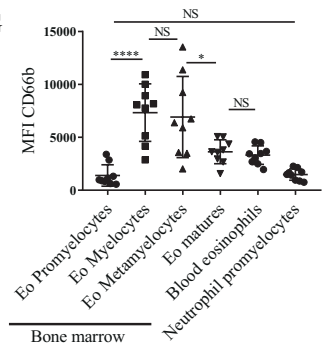

E

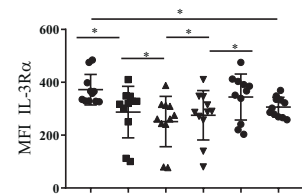

H

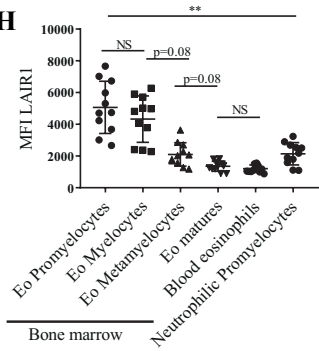

$\mathbf{F}$

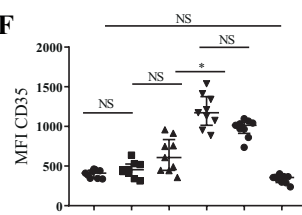

I

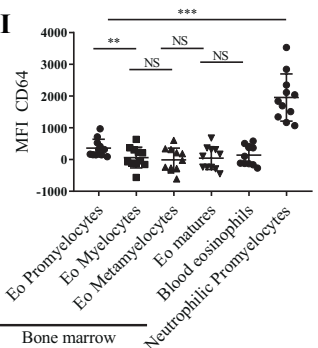

\section{$\mathbf{J}$}

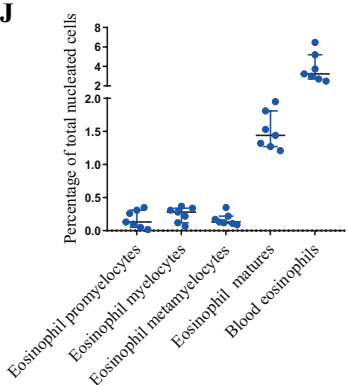

Figure 2. Expression of surface markers on eosinophil progenitors and mature cells.

Cells are gated like shown in Figure 1. Eosinophilic promyelocytes are CD11b and CD62L, eosinophilic myelocytes are $\mathrm{CD}_{11} \mathrm{~b}^{+}$and $\mathrm{CD} 62 \mathrm{~L}$; eosinophilic metamyelocytes are $\mathrm{CD} 11 \mathrm{~b}^{+}$and $\mathrm{CD} 6 \mathrm{~L}^{\mathrm{dim}}$ and mature eosinophils (in blood and BM) are CD11 b+ and CD62L'. Median fluorescence intensity (without subtraction of FMO or isotype's fluorescence ) is plotted for the different surface markers of eosinophils in $\mathrm{BM}$ and blood, and their progenitors in the BM at baseline. Neutrophilic promyelocytes have been added as a negative control as a comparator. A. CCR3 (CD193), B. Siglec-8, C. CD49d, D. IL-5R (CD125), E. IL-3R $\alpha$ (CD123), F. CR1 (CD35), G. CEACAM-8 (CD66b), H. LAIR1 (CD305), I. FcgRI (CD64). J. The percentage of total nucleated cells for the eosinophil precursors and eosinophils in blood and bone marrow is depicted. All results are depicted as medians \pm IQR. A Friedman test with a uncorrected Dunn's test was performed. NS: not significant $* P \leq 0.05,{ }^{* *} \mathrm{P} \leq 0.01$ and ${ }^{* * *} \mathrm{P} \leq 0.001$. 


\section{Acknowledgments}

The authors thank Manita Bremmers, the physician assistant who performed the bone marrow aspirations. 


\section{References}

1. Meeusen EN, Balic A. Do eosinophils have a role in the killing of helminth parasites? Parasitol Today 2000;16(3):95-101.

2. Yazdanbakhsh M, Tai PC, Spry CJ, Gleich GJ, Roos D. Synergism between eosinophil cationic protein and oxygen metabolites in killing of schistosomula of Schistosoma mansoni. J Immunol 1987;138(10):3443-7.

3. Xiao Z, Zhang Y, Wang J, Hao Y. Study on Clinical and Laboratory Characteristics of Chronic Eosinophilic Leukemia CEL/Hypereosinophilic Syndrome (CEL/HES). Blood;110(11):.

4. Sehmi R, Wood LJ, Watson R, et al. Allergen-induced increases in IL-5 receptor alphasubunit expression on bone marrow-derived CD34+ cells from asthmatic subjects. A novel marker of progenitor cell commitment towards eosinophilic differentiation. J Clin Invest 1997;100(10):2466-2475.

5. Mori Y, Iwasaki H, Kohno K, et al. Identification of the human eosinophil lineage-committed progenitor: revision of phenotypic definition of the human common myeloid progenitor. J Exp Med 2009;206(0022-1007 (Linking)):183-193.

6. Loken MR, van de Loosdrecht A, Ogata K, Orfao A, Wells DA. Flow cytometry in myelodysplastic syndromes: Report from a working conference. Leuk Res 2008;32(1):5-17.

7. Buitenhuis M. Cell Signalling During Human Eosinophil Differentiation. In: Methods in molecular biology (Clifton, N.J.) p53-70.

8. Höchstetter R, Dobos G, Kimmig D, Dulkys Y, Kapp A, Elsner J. The CC chemokine receptor 3 CCR3 is functionally expressed on eosinophils but not on neutrophils. Eur J Immunol 2000;30(10):2759-2764.

9. Floyd $\mathrm{H}, \mathrm{Ni} \mathrm{J}$, Cornish AL, et al. Siglec-8. A novel eosinophil-specific member of the immunoglobulin superfamily. J Biol Chem 2000;275(2):861-6.

10. Lund-Johansen F, Terstappen LWMM. Differential surface expression of cell adhesion molecules during granulocyte maturation. J Leukoc Biol 1993;54(1):47-55.

11. Ahearn JM, Fearon DT. Structure and function of the complement receptors, CR1 (CD35) and CR2 (CD21). Adv Immunol 1989;46183-219.

12. Verbrugge A, de Ruiter T, Geest C, Coffer PJ, Meyaard L. Differential expression of leukocyteassociated Ig-like receptor-1 during neutrophil differentiation and activation. J Leukoc Biol 2006;79(4):828-836.

13. Kerst JM, van de Winkel JG, Evans AH, et al. Granulocyte colony-stimulating factor induces hFc gamma RI (CD64 antigen)-positive neutrophils via an effect on myeloid precursor cells. Blood 1993;81(6):1457-64.

14. Schudel. L. Leitfaden der Blutmorphologie: Manual of blood morphology. 11 edn. Zurich: Thieme 1965.

15. Cartwright GE, Athens JW, Wintrobe MM. The kinetics of granulopoiesis in normal man. Blood 1964;24780-803. 
A

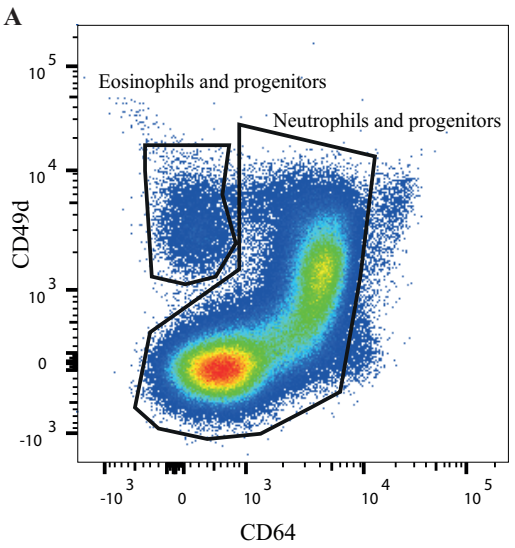

Eosinophil lineage

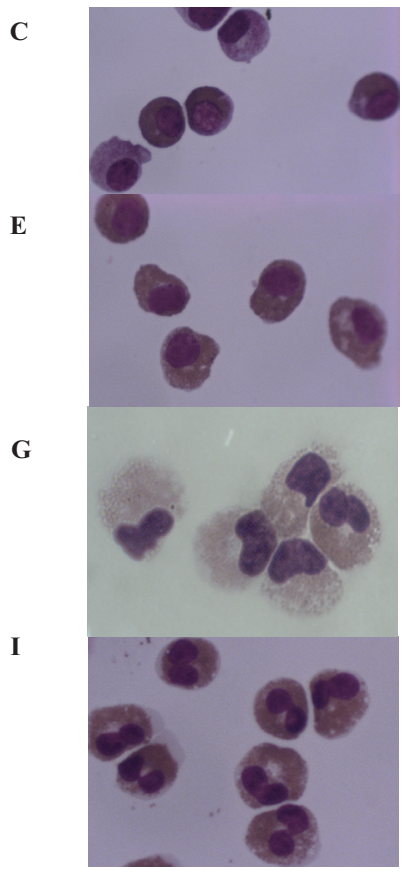

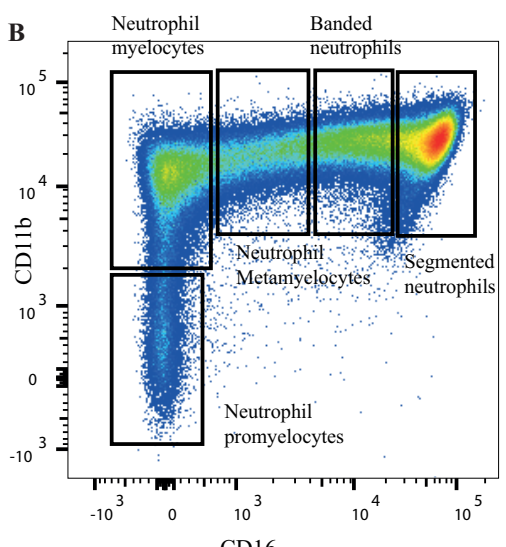

CD16

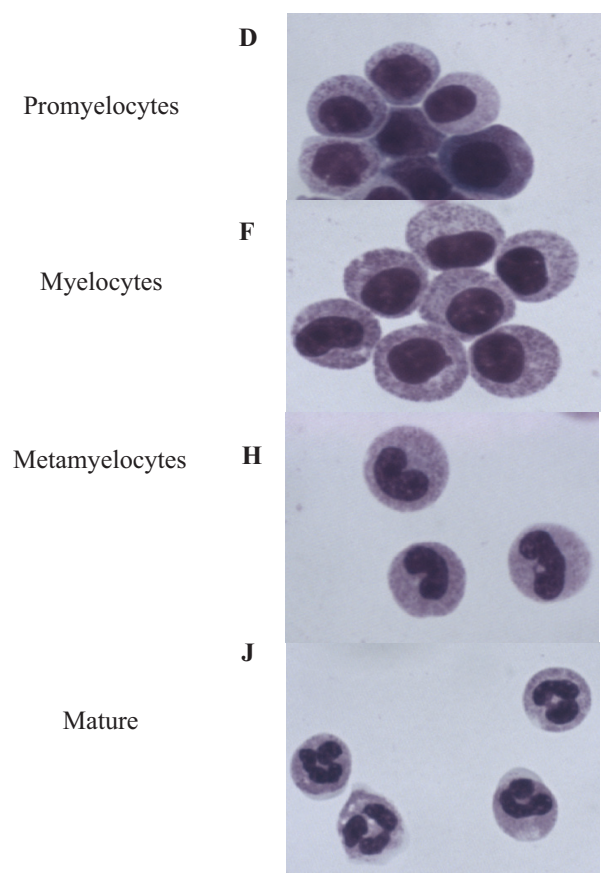

Supplementary Figure 1. Alternative gating of eosinophil and neutrophils and their progenitors in the bone marrow.

A. An example of gating eosinophils and neutrophils and their progenitors is shown. This plot was obtained after gating cells subsequently for singlets, excluding debris, high SSC and excluding CD64 ${ }^{++}$ like is shown in Figure 1. However CD64 and CD49d is now used instead of CD193 to distinguish both neutrophil and eosinophil lineages.

B. After gating all neutrophils and their progenitors as is shown in Supplementary Figure $1 \mathrm{~A}$, neutrophil precursors are gated like described before. ${ }^{6}$ Neutrophilic promyelocytes are $\mathrm{CD}^{-} 6^{-}$and $\mathrm{CD}_{11 \mathrm{~b}}$, 
neutrophilic myelocytes are $\mathrm{CD}_{16}{ }^{-}$and $\mathrm{CD} 11 \mathrm{~b}^{+}$, neutrophil metamyelocytes are $\mathrm{CD} 16^{\mathrm{dim}}$ and $\mathrm{CD} 11 \mathrm{~b}^{+}$, neutrophil bandeds are $\mathrm{CD} 16^{+}$and $\mathrm{CD} 11 \mathrm{~b}^{+}$and mature neutrophils are $\mathrm{CD} 16^{++}$and $\mathrm{CD} 11 \mathrm{~b}^{+}$.

C-J. Representative cytospins of sorted neutrophilic and eosinophilic precursors and mature cells are shown after staining with May-Grünwald-Giemsa (Objective used: 100x, Axioskop 40, Light microscopy, Carl Zeiss, Goettingen, Germany). The distinction between eosinophilic promyelocytes (C) and myelocytes (E) and neutrophilic promyelocytes (D) and myelocytes (F) can be clearly seen as eosinophilic precursors contain the for eosinophils specific (dark) orange granules that are lacking in the neutrophilic precursors. 

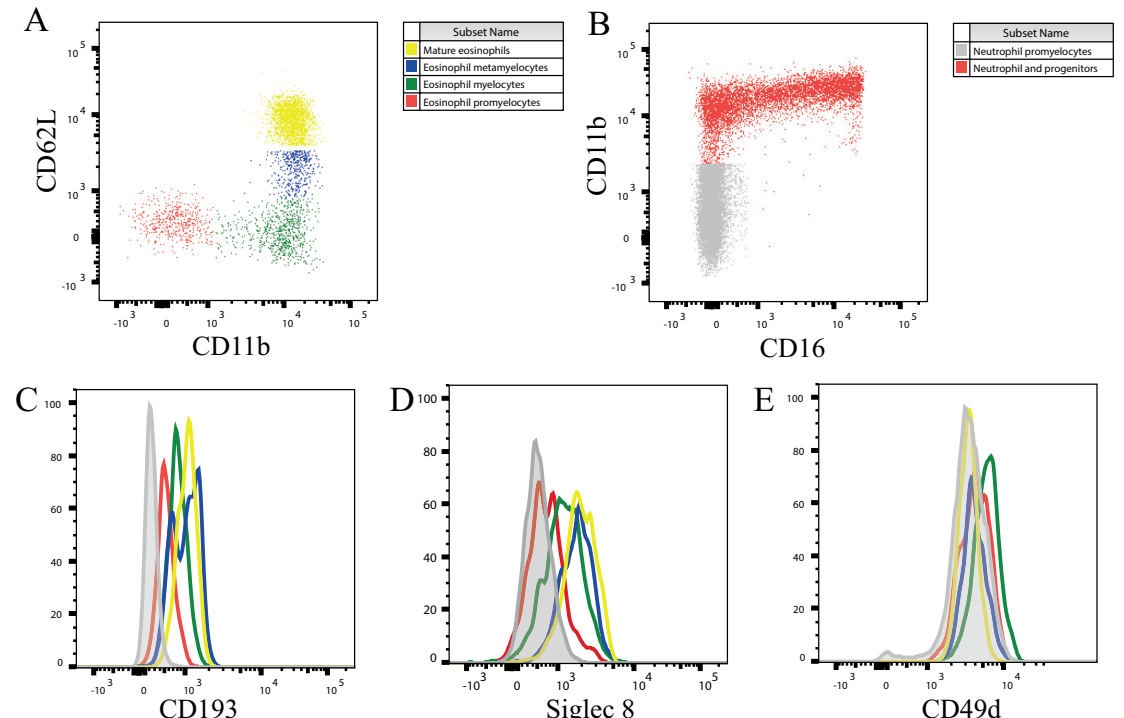

F

G
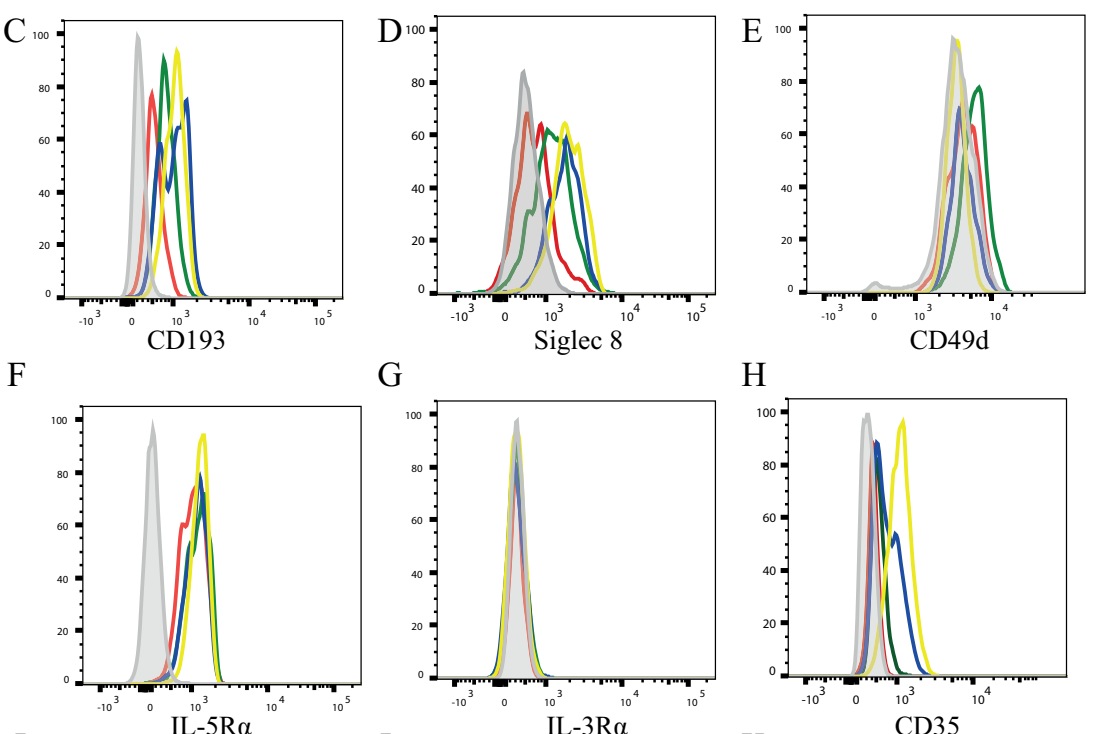

$\mathrm{H}$
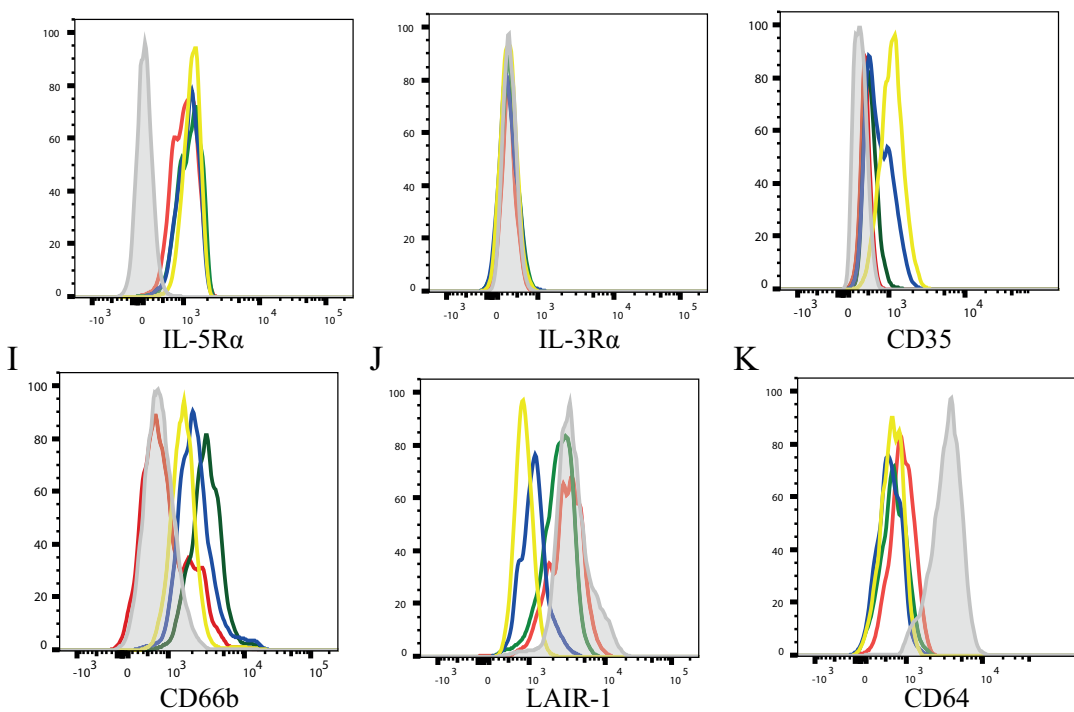

K
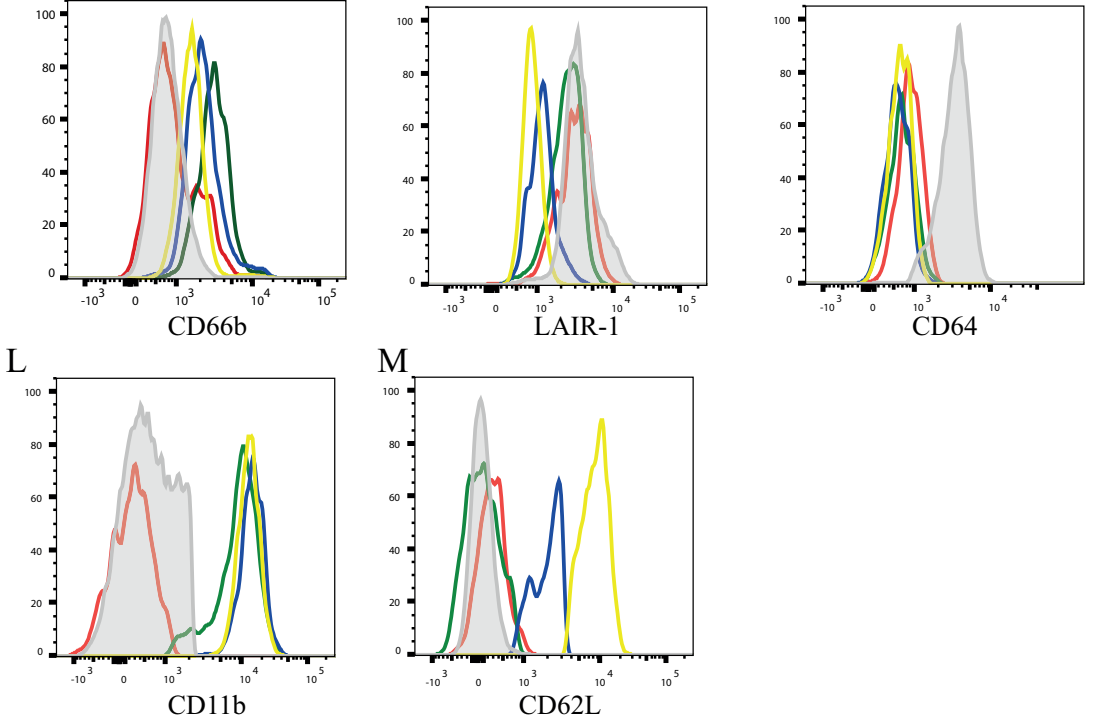
Supplementary Figure 2. Representative histograms for all measured expression markers on the different eosinophil maturation stages and neutrophilic promyelocytes.

A-B. Eosinophils and their progenitors are gated like shown in Figure 1 and neutrophilic promyelocytes are gated like shown in Supplementry Figure 1. Yellow histograms depict mature eosinophils, blue depict eosinophilic metamyelocytes, green depict eosinophilic myelocytes, red depict eosinophilic promyelocytes and grey depict neutrophilic promyelocytes. C. CCR3 (CD193), D. Siglec-8, E. CD49d, F. IL-5R $\alpha$ (CD125), G. IL-3R $\alpha$ (CD123), H. CR1 (CD35), I. CEACAM-8 (CD66b), J. LAIR1 (CD305), K. FcgRI (CD64), L. MAC-1 (CD11b), M. L-Selectin (CD62L). 


\section{Chapter 8}

\section{Differentiation and activation of eosinophils in the human bone marrow during experimental human endotoxemia}

Marwan Hassani, $M D^{1,2}$, Guus Leijte, $M D^{3}$, Niklas Bruse, MSc ${ }^{3}$, Matthijs Kox, PhD', Peter Pickkers, $M D, P h D^{3}$, Nienke Vrisekoop, $P h D^{1,2}$ and Leo Koenderman, $P h D^{1,2}$

1. Department of Respiratory Medicine, University Medical Centre Utrecht, Utrecht, the Netherlands.

2. Laboratory of Translational Immunology, University Medical Centre Utrecht, Utrecht, the Netherlands. 3. Department of Intensive Care and Radboud Center for Infectious Diseases, Radboud University Medical Center, Nijmegen, The Netherlands 


\section{Abstract}

Acute infection is characterized by eosinopenia. However, the underlying mechanism(s) are poorly understood and it is unclear whether decreased mobilization/production of eosinophils in the bone marrow (BM) and/or increased homing to the tissues play a role.

The objective of this study was to investigate the differentiation and activation status of eosinophils in the human BM and blood upon experimental human endotoxemia, a standardized, controlled and reproducible model of acute systemic inflammation.

A BM aspirate and venous blood was obtained from 7 healthy volunteers before, four hours after, and one week after intravenous challenge with $2 \mathrm{ng} / \mathrm{kg}$ endotoxin. Early progenitors (CD34+/ IL-5R $\alpha+$ ), eosinophil promyelocytes, myelocytes, metamyelocytes and mature eosinophils were identified and quantified in the bone marrow and blood samples using flowcytometry based on specific eosinophil markers (CD193 and IL-5R $\alpha$ ). Activation status was assessed using antibodies against known markers on eosinophils: Alpha-4 (CD49d), CCR3 (CD193), CR1 (CD35), CEACAM- 8 (CD66b), CBRM 1/5 (activation epitope of MAC-1) and by plasma cytokine analysis.

Four hours after endotoxin administration, numbers of mature eosinophils in the blood and in the BM markedly declined compared with baseline, whereas numbers of all eosinophil progenitors did not change. The remaining eosinophils did not show signs of activation or degranulation despite significantly increased circulating levels of eotaxin-1. Furthermore, the expression of CD49d and CD193 on eosinophils was lower compared to baseline, but normalized after 7 days.

Together these data imply that circulatory eosinopenia after an innate immune challenge is mediated by CD49d mediated homing of eosinophils to the tissues. 


\section{Introduction}

Eosinophils are cytotoxic granulocytes that are typically involved in host defense against multicellular parasites ${ }^{1}$. Apart from their immune function, eosinophils are also thought to be important for homeostatic functions ${ }^{2}$, such as maintenance of metabolic homeostasis ${ }^{3}$, tissue remodeling ${ }^{4}$ and T-cell selection in the thymus ${ }^{5}$. On the other hand, eosinophils play a pathological role in diseases such as asthma and eosinophilic esophagitis ${ }^{6,7}$. In asthma these cells are recruited to the airways after allergic or nonallergic stimuli and are responsible for bronchial airway inflammation ${ }^{8}$. In eosinophilic esophagitis, eosinophils are present in the esophageal tissue but their role in disease pathogenesis remains unresolved ${ }^{7}$. Most of these Type 2 (T2) driven diseases are accompanied with eosinophilia in the circulation ${ }^{9,10}$.

In contrast, eosinophil numbers are markedly reduced in response to bacterial infection ${ }^{11}$. This phenomenon was initially believed to result from enhanced release of adrenal glucocorticoids ${ }^{12}$. However, adrenalectomized mice subjected to acute pneumococcal infection still displayed eosinopenia in the absence of enhanced circulating corticosterone levels ${ }^{11}$. Therefore, the disappearance of eosinophils from the circulation must be mediated by a different mechanism. So far, this mechanism has not been elucidated and especially hard to address in humans. Understanding of the underlying mechanism might teach us more about type 2 immunity, since there is clear cross-talk between T2 immunity and innate immune responses. This is clearly illustrated by studies showing that exposure to environmental LPS can affect the clinical course of allergic diseases, including asthma ${ }^{13}$. In accordance, it was demonstrated that CD14, a co-receptor for LPS, is one of the risk genes for asthma ${ }^{14}$.

We have previously published that experimental human endotoxemia (a model of acute systemic inflammation in humans in vivo) results in eosinopenia within 2 hours after endotoxin administration ${ }^{15}$. However, we did not establish whether this was due to decreased mobilization/production of eosinophils in the bone marrow (BM) or a result of increased homing from the blood to the tissues. In the present study, we investigated the maturation and activation status of eosinophils in the BM and blood compartments of healthy volunteers during homeostasis and during experimental endotoxemia in order to delineate the mechanisms underlying the previously observed eosinopenia. These data were complemented by determining eosinophil modulating cytokines and chemokines in blood plasma.

\section{Materials and methods}

\section{Ethics, subjects and study design}

In this study, we included 7 healthy male volunteers who participated in a human endotoxemia study performed at the Radboud university medical center in Nijmegen, the Netherlands (ref. no. NL61136.091.17). This study was approved by the local ethics review board (CMO Arnhem-Nijmegen; reference no. 2017-3607) and was conducted in compliance with declaration of Helsinki (Forteleza, 2013); International Conference on Harmonisation Good Clinical Practice guidelines, and the rulings of the Dutch Medical Research Involving Human Subjects Act. Written informed consent was obtained from 
all study participants. All individuals were thoroughly screened and found to be healthy. Among the exclusion criteria were use of medication, history or signs of atopic disease, and signs of acute infection. All subjects underwent an intravenous endotoxin challenge according to a standardized protocol described in detail elsewere ${ }^{16}$. In short, a single dose of $2 \mathrm{ng}$ per kilogram of bodyweight lipopolysaccharide (LPS, derived from Escherichia coli O:113, Lot \#94332B1, National Institutes of Health, Bethesda, MD) was injected ( $t=0$ hours) to induce a controlled systemic inflammatory response. Bone marrow and blood were sampled at three timepoints: at baseline ( 7 days before endotoxin administration), 4 hours after endotoxin administration, and in 6 out of 7 volunteers 7 days after endotoxin administration (Figure 1).

\section{Sample processing}

Blood was collected in sodium heparin tubes (Vacuette ${ }^{\circledR}$ Greiner bio-one, Kremsmünster, Austria) and bone marrow aspirates were collected in syringes containing a sodium heparin solution (3:1 ratio). Thereafter, erythrocytes were lysed using an ice cold isotonic lysis buffer consisting of $150 \mathrm{mM} \mathrm{NH}_{4} \mathrm{Cl}, 10 \mathrm{mM} \mathrm{KHCO}_{3}$ and $0.1 \mathrm{mM} \mathrm{NA}_{2}$ EDTA. Leukocytes were washed and resuspended in a staining buffer consisting of PBS supplemented with $0.32 \% \mathrm{w} / \mathrm{v}$ trisodium citrate and $10 \% \mathrm{w} / \mathrm{v}$ human plasma albumin solution. Samples were immediately transported on ice to the UMCU and further processed within 90 min.

For determination of plasma cytokines, ethylenediaminetetraacetic acid (EDTA) anticoagulated blood were centrifuged directly after withdrawal $\left(2000 \times \mathrm{g}\right.$ at $4{ }^{\circ} \mathrm{C}$ for 10 $\mathrm{min})$, and stored at $-80^{\circ} \mathrm{C}$ until further analysis.

\section{Flow cytometry}

One million cells were stained with antibodies for 30 minutes at a concentration of 40 million cells per ml on ice in the UMCU and washed twice before analysis on the LSR Fortessa flow cyometer (BD, Mountain View CA, USA). An overview of the antibodies that were used for staining is provided in Supplementary Table 1. Eosinophils and their progenitors in blood and bone marrow were identified as published before (supplementary Figure $1 \mathrm{~A}$ and $1 \mathrm{~B})^{17}$. Also early eosinophil progenitors (EoPs) were identified as was published before (supplementary Figure 1C and 1D) ${ }^{18}$.

\section{Cytokine analysis}

Cytokine concentrations were determined in one batch using a multiplex assay (Milliplex, Millipore, Billerica, MA, USA) according to the manufacturers' guidelines.

\section{Data analysis and representation}

FlowJo v10 (LLC, Ashland, OR, USA) was used to analyze the flow cytometry data and to determine the different median fluorescence intensities. Statistical analysis was performed using GraphPad Prism 7.04 (Graphpad Software, La Jolla, CA, USA). Data are presented as median [interquartile range]. A Wilcoxon matched-pairs signed rank test was performed to compare two groups. A p-value of less than 0.05 was considered statistically significant. 


\section{Results and Discussion}

\section{Subject characteristics}

Blood and BM samples were collected from 7 healthy male volunteers at baseline (day 0 ) and 4 hours after endotoxin administration (day 7), and from 6 volunteers one week after endotoxin administration (day 14, Figure 1A). Age was 23 [19-28] years (median, [Q1-Q3]), weight 85 [85-87] kg, height 1.84 [181-185] m and BMI 25.0 [24.8-25.9] kg/ $\mathrm{m}^{2}$. Blood eosinophil count at baseline was $0.19[0.17-0.26] \times 10^{6} / \mathrm{ml}$. No adverse effects occurred during the conduct of the study.

\section{$\mathbf{A}$}

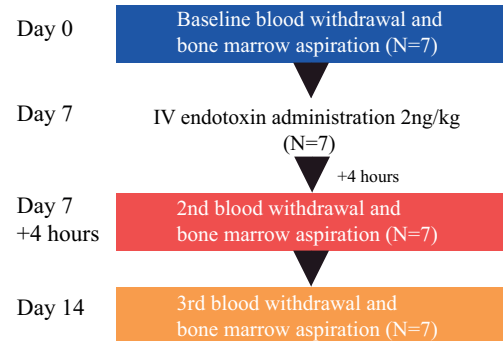

B

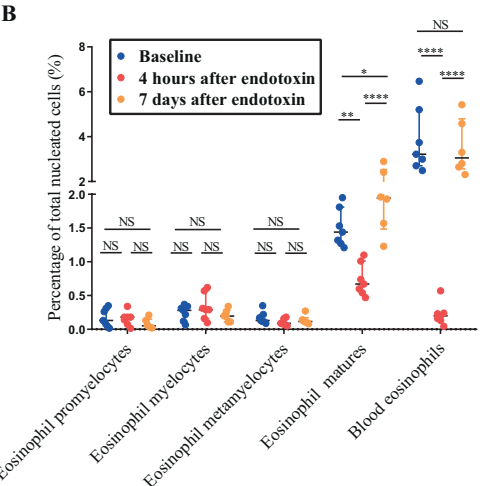

E
C

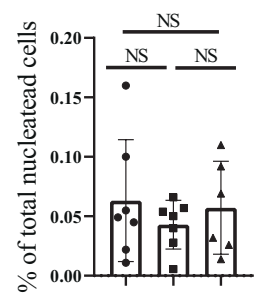

को को के के
D
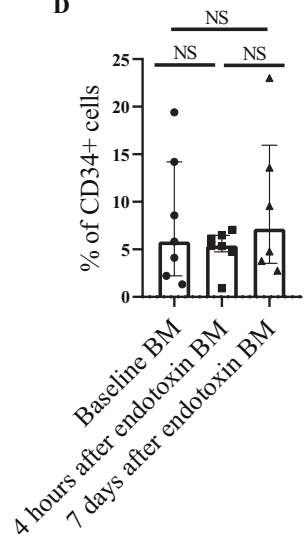
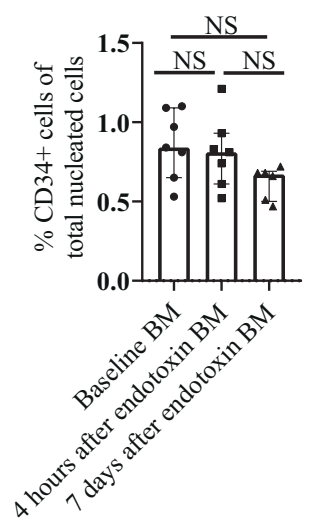

Figure 1. Study flow chart and the number of eosinophils and their progenitors after endotoxin challenge.

A. Study design. 7 healthy volunteers underwent a baseline bone marrow aspiration and vena puncture (blue). 7 volunteers were challenged with an intraveneous dose of $2 \mathrm{ng} / \mathrm{kg}$ lipopolysaccharide (LPS) at day 7 and a $2^{\text {nd }}$ bone marrow aspiration and blood withdrawal 4 hours later (red). Finally, 6 volunteers underwent a final bone marrow aspiration and blood withdrawal at day 14 (orange).

B. Number of eosinophils in the bone marrow and blood, and their progenitors (from eosinophil promyelocyte stage and further) in the bone marrow are depicted as a percentage of total nucleated cells. C. Number of EoPs (CD34+ and IL-5R $\alpha+$ cells) in the bone marrow are depicted as a percentage of total nucleated cells or $\mathbf{D}$. as a percentage of CD34+ cells. 
E. Number of CD34+ cells are depicted as a percentage of total nucleated cells.

Results are plotted as median \pm IQR. A Wilcoxon matched-pairs signed rank test was performed. NS: not significant. ${ }^{*} \mathrm{P} \leq 0.05^{* *} \mathrm{P} \leq 0.01$ and ${ }^{* * * *} \mathrm{P} \leq 0.0001$.

\section{Decrease of the number of mature eosinophils in bone marrow and blood during experimental human endotoxemia}

We have previously shown that the number of eosinophils in the circulation markedly decrease during experimental human endotoxemia within 2 hours $^{15}$. This decrease remained present for at least 8 hours and was restored to baseline levels after 24 hours $^{15}$. To determine whether this eosinopenia is mainly a result of decreased production in the bone marrow or increased homing to the tissue, we studied the effect of endotoxin administration on eosinophils and their progenitors in the BM. At baseline, the median percentages of total nucleated cells [Q1-Q3] of promyelocytes, myelocytes, metamyelocytes and mature eosinophils in the BM were $0.13 \%$ [0.05-0.31\%] 0.28\% [0.12-0.34\%] 0.22\%, [0.11-0.35\%] and 1.4\% [1.3-1.8\%], respectively (Figure 1B). The median percentage of blood eosinophils at baseline was $3.2 \%$ [2.7-5.2\%]. Four hours after endotoxin administration, the percentage of progenitors (promyelocytes, myelocytes and metamyelocytes) in BM did not significantly change (Figure 1B). Also, the number of early eosinophil progenitors (EoPs) as a percentage of total white blood cells (WBC) or as a percentage of total $\mathrm{CD} 34+$ cells in the BM did not change (Figure $1 \mathrm{C}$ and $1 \mathrm{D}$ respectively). Furthermore, the proportion of BM CD34+ cells within the total WBC was also not attenuated after endotoxin administration. However, one should take into account that only one time point ( $t=4$ hours) was measured and changes might have occurred at other moments. On the other hand, in our earlier work, we have established that the number of eosinophils in the circulation was already restored within 24 hours after endotoxin administration, which also suggested no alterations in BM eosinophilopoiesis ${ }^{15}$.

In contrast, the percentage of mature eosinophils in the BM decreased significantly to $0.67 \%[0.54-1.0 \%]$, albeit to a lesser extent than in the circulation which showed a decrease to $0.20 \%$ [0.13-0.24\%] (see figure 1B). The absolute number of mature eosinophils also decreased in blood and bone marrow after endotoxemia despite an increase of the total white blood cell count in the peripheral blood (Supplementary Figure 2). One week after the endotoxin challenge, the number of cells both in the circulation and in the BM was normalized (Figure 1B). So, endotoxin administration most likely resulted in mobilization of only the mature eosinophils from the BM to the circulation. The situation in the peripheral blood was more complex and likely mediated by an additional mechanism: cells may disappear from the circulation due to increased margination in e.g. the sinusoids of liver and spleen, ${ }^{19,20}$ or by transmigration and homing into distant tissues such as gut and adipose tissue (see review) ${ }^{2}$. Indeed, more circulatory eosinophils transmigrated into the tissue of patients with asthma in response to a bronchial segmental challenge with endotoxin, ${ }^{21}$ which is possibly initiated by the release of chemoattractants by neutrophils. ${ }^{22}$ 
A

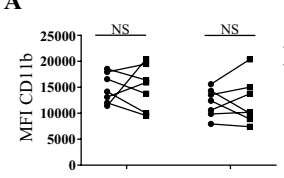

D
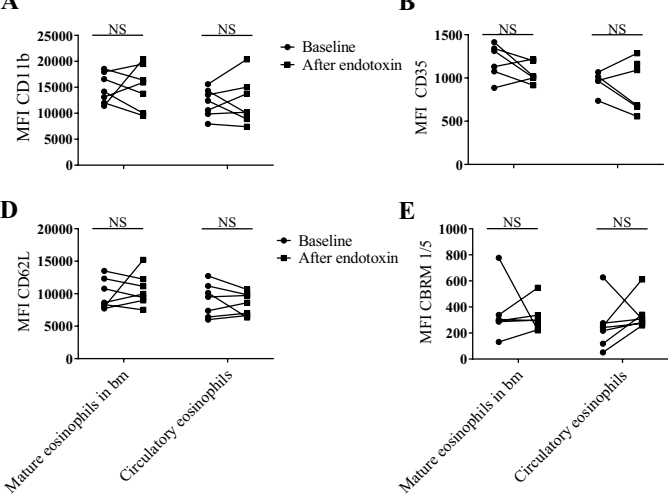

B

(

$\mathbf{E}$

E

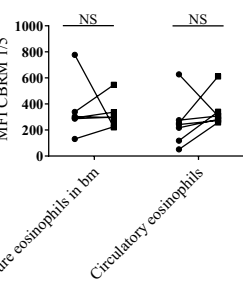

(20)

C

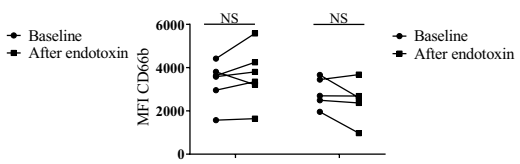

- Baseline

F

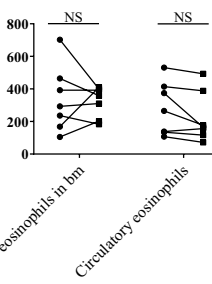

- Baseline

$\rightarrow$ After endotoxir

G

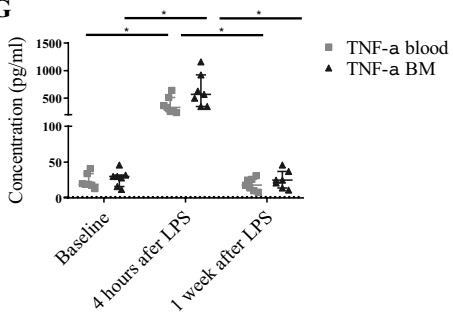

Figure 2. Change of surface markers on mature eosinophils in the BM and circulation

after endotoxin administration. Median fluorescence intensity is plotted for the different surface markers of mature eosinophils in bone marrow and blood at baseline and 4 hours after endotoxin.

A.CD11b. B.CD35. C. CD66b. D. CD62L and E. CBRM 1/5 (antibody that only recognizes the activated epitope of Mac-1) F. CD64. Individual data points are shown. G. Level of TNF- $\alpha$ in plasma and bone marrow supernatant is plotted for the different time points (baseline, 4 hours after endotoxemia and 1 week after endotoxemia). Median with interquartile range is indicated.

A Wilcoxon matched-pairs signed rank test was performed. NS: not significant, $* P \leq 0.05$.

Effect of acute experimental inflammation on surface markers of eosinophils To address whether endotoxin leads to activation or degranulation of eosinophils in the $\mathrm{BM}$ or blood, a selection of surface markers was measured: CD11b, CD35, CD66b, CD62L and CBRM 1/5 (recognizes an activation epitope of MAC-1). No differences in marker expression on the different eosinophil precursors were measured during experimental endotoxemia (Supplementary Figure 3). The same was true for most surface proteins on mature eosinophils in the BM and circulation (Figure 2). We have also measured activation markers on mature eosinophils in the circulation of 6 new donors at baseline and after endotoxemia on a fast ( $<25 \mathrm{~min}$ ) and fully automated flow cytometer (Aquios, Beckman Coulter, Pasadena, CA, USA) as was described in detail before ${ }^{23}$ in order to decrease the effects of in vitro manipulation of cells to a minimum. Again no change in expression of activation markers after endotoxemia was found (Supplementary Figure 4 ), indicating that in vitro manipulation of eosinophils did not play a significant role in our protocol. 
Therefore, blood and BM eosinophils did not seem to exhibit an activated or degranulated phenotype during endotoxemia. This is surprising, as TNF- $\alpha$ increased after endotoxin administration in blood and in the bone marrow (see Figure 2G) ${ }^{24}$. This cytokine can stimulate eosinophils in vitro which can lead to degranulation ${ }^{25}$. However, the concentration of TNF- $\alpha$ used in vitro $(100 \mathrm{ng} / \mathrm{ml})$ was much higher than the concentration reached in vivo $(0.5 \mathrm{ng} / \mathrm{ml} \text {, see Figure } 2 \mathrm{G})^{24,25}$.

On the other hand, following endotoxin administration some other markers showed slight but significant alterations. For instance, CD193 expression on mature eosinophils in the BM was attenuated after endotoxin challenge (Figure 3A). This difference was not observed in the circulation (Figure $3 A$ ). One week after endotoxin challenge, expression of CD193 on BM mature eosinophils returned to normal levels (Figure 3A). In an earlier study we have found that CD193 is slightly upregulated upon maturation of eosinophils in the bone marrow ${ }^{17}$. So, a possible explanation as to why CD193 expression is reduced after endotoxin administration, is 'rejuvenation' of the BM compartment, since it is plausible that only the most mature eosinophils are released into the circulation during acute inflammation. The unchanged CD193 expression on circulatory eosinophils supports this hypothesis.

Additionally, we found that the expression of CD49d on only circulatory eosinophils was also attenuated after endotoxin administration (Figure 3B). This decrease returned to normal after one week. The decrease in CD49d expression on blood eosinophils may imply that CD49d is involved in the recruitment of eosinophils to tissues, leaving behind the cells with the lowest expression as also suggested by the work of Johansson and colleagues $^{26}$. This is also supported by the finding that treatment of human umbilical vein endothelial cells (HUVEC cells) with LPS resulted in adherence of eosinophils to these cells via the interaction between VLA-4 (integrin dimer composed of CD49d and CD29) and VCAM-127.

\section{Effect of endotoxin administration on plasma cytokine levels}

The eosinophil specific chemokines, eotaxin 1, 2 and 3 were measured in plasma at baseline, 4 hours and 1 week after experimental endotoxemia. Eotaxin 3 was not detectable at all 3 time points (Figure 3C). Eotaxin 1 and 2 both increased in blood following endotoxemia, but this increase was only statistically significant for eotaxin-1 (Figure $3 C$ ), although eotaxin-2 showed an obvious trend $(p=0.078)$. Both chemokines returned to baseline level after 1 week (Figure $3 C$ ). Since, we also observed a decrease in CD49d expression on circulatory eosinophils (Figure 3B), it is tempting to speculate that systemic endotoxin challenged led to eosinophil transmigration via VLA-4 to the tissues in response to local production of eotaxin-128. In contrast, L-selectin did not seem to be involved in transmigration of eosinophils as its expression did not change (Figure 2D and Supplementary Figure 4C). This is different from the role of L-Selectin in neutrophils where it has been shown that LPS can activate neutrophils through this receptor. ${ }^{29}$ 
A

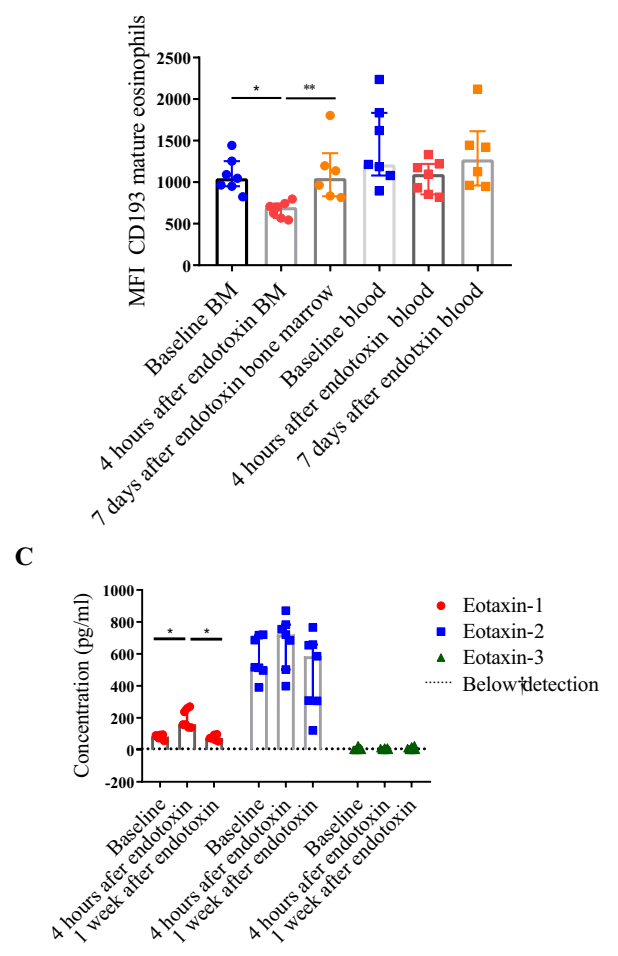

B

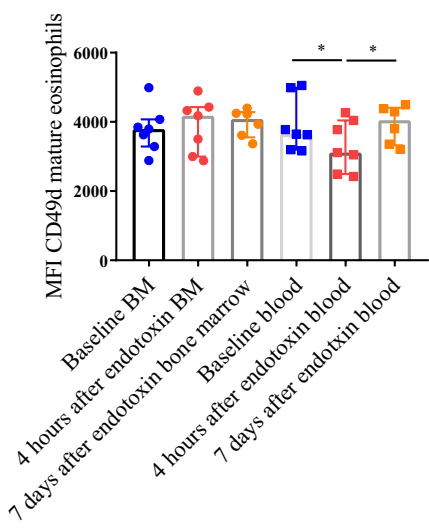

D

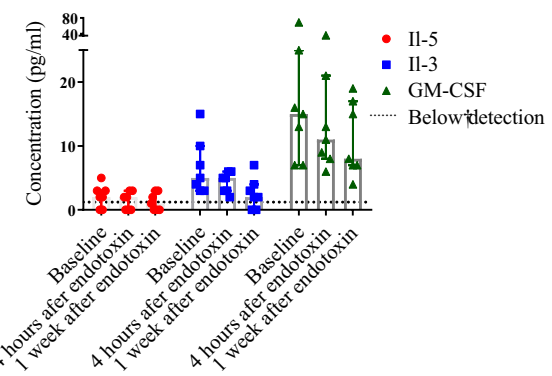

Figure 3. Expression of CD193 and CD49d a on mature eosinophils and cytokine concentration in plasma before and after endotoxin.

Median fluorescence intensity (MFI) is plotted for two different surface markers of mature eosinophils in bone marrow and blood at baseline (blue), 4 hours after endotoxin administration (red) and 1 week after endotoxin administration (orange): A. CD193, B. CD49d. C. Eotaxin 1-3 concentrations in the plasma are indicated. Red shows eotaxin-1, blue eotaxin-2 and green eotaxin-3. D. IL-5, IL-3 and GM-CSF concentrations in the plasma are also indicated. Red IL-5, blue IL-3 and green GM-CSF. Medians \pm IQR are indicated. A Wilcoxon matched-pairs signed rank test was performed. All conditions were tested, but only statistical significant results are indicated.

${ }^{*} \mathrm{P} \leq 0.05$ and ${ }^{* *} \mathrm{P} \leq 0.01$.

The plasma levels of IL-3, IL-5 and GM-CSF were close to their detection limits in the plasma during steady state (Figure 3D). Following 4 hours and 1 week after endotoxemia, the level of these cytokines did not show evident alterations (Figure 3D).

Our findings implicate that the eosinophil compartment is influenced by endotoxin induced systemic inflammation beyond the Th2/ILC2 induced allergic type of response. This non-allergic mechanism that is present during endotoxin-induced innate immune responses might be important in homing of eosinophils to tissues that contain these cells also in homeostasis such as gut ${ }^{30}$, uterus ${ }^{31}$, thymus ${ }^{5}$ and adipose tissue. ${ }^{3}$ It is intuitive that such homeostatic homing is not associated with cytotoxic activation of 
the cells as the risk for collateral damage to the tissue is too large. Similar mechanisms might also contribute to recruitment of eosinophils to tissues in allergic diseases as the gene for the LPS co-receptor CD14 is a risk gene for allergic asthma. ${ }^{14}$ However, in our model it is unknown whether LPS can interact with the airway epithelium. Therefore, the mechanisms induced by LPS induced systemic inflammation might not be part of the mechanisms operational in allergic disease where epithelial derived cytokines/ alarmins are involved in the control of eosinophil homing from the periphery to the airways.

In conclusion, we propose a model in which experimental human endotoxemia induces a partial mobilization of mature eosinophils from the BM into the circulation and a large mobilization of circulatory eosinophils into tissues without signs of activation, degranulation or enhanced production of eosinophil precursors. This mechanism is probably regulated through VLA-4 and eotaxin-1.

\section{Acknowledgments}

The authors thank Manita Bremmers, the physician assistant who performed the bone marrow aspirations. The authors thank Jelle Gerretsen of the department of intensive care medicine Nijmegen for his help during the preparation of the samples. 


\section{References}

1. Huang L, Appleton JA. Eosinophils in Helminth Infection: Defenders and Dupes. Trends Parasitol [Internet]. 2016 [cited 2019 Sep 23];32:798-807. Available from: http://www.ncbi. nlm.nih.gov/pubmed/27262918

2. Marichal T, Mesnil C, Bureau F. Homeostatic Eosinophils: Characteristics and Functions. Front Med [Internet]. 2017 [cited 2018 Oct 8];4:101. Available from: http://www.ncbi.nlm. nih.gov/pubmed/28744457

3. Wu D, Molofsky AB, Liang H-E, Ricardo-Gonzalez RR, Jouihan HA, Bando JK, et al. Eosinophils Sustain Adipose Alternatively Activated Macrophages Associated with Glucose Homeostasis. Science (80- ) [Internet]. 2011 [cited 2018 Jul 31];332:243-7. Available from: http://www. ncbi.nlm.nih.gov/pubmed/21436399

4. Lacy P, Moqbel R. Immune effector functions of eosinophils in allergic airway inflammation. Curr Opin Allergy Clin Immunol [Internet]. 2001 [cited 2018 Jul 31];1:79-84. Available from: http://www.ncbi.nlm.nih.gov/pubmed/11964674

5. Throsby M, Herbelin A, Pléau JM, Dardenne M. CD11C+ eosinophils in the murine thymus: developmental regulation and recruitment upon MHC class I-restricted thymocyte deletion. J Immunol [Internet]. 2000 [cited 2018 Jul 31];165:1965-75. Available from: http://www. ncbi.nlm.nih.gov/pubmed/10925279

6. Gleich GJ. The eosinophil and bronchial asthma: current understanding. J Allergy Clin Immunol [Internet]. 1990 [cited 2018 Jul 27];85:422-36. Available from: http://www.ncbi. nlm.nih.gov/pubmed/2406322

7. Blanchard C, Stucke EM, Rodriguez-Jimenez B, Burwinkel K, Collins MH, Ahrens A, et al. A striking local esophageal cytokine expression profile in eosinophilic esophagitis. J Allergy Clin Immunol [Internet]. 2011 [cited 2018 Jul 27];127:208-217.e7. Available from: http:// www.ncbi.nlm.nih.gov/pubmed/21211656

8. McBrien CN, Menzies-Gow A. The Biology of Eosinophils and Their Role in Asthma. Front Med [Internet]. 2017 [cited 2018 Jul 31];4:93. Available from: http://www.ncbi.nlm.nih. gov/pubmed/28713812

9. Konikoff MR, Blanchard C, Kirby C, Buckmeier BK, Cohen MB, Heubi JE, et al. Potential of Blood Eosinophils, Eosinophil-Derived Neurotoxin, and Eotaxin-3 as Biomarkers of Eosinophilic Esophagitis. Clin Gastroenterol Hepatol [Internet]. 2006 [cited 2019 Sep 23];4:1328-36. Available from: http://www.ncbi.nlm.nih.gov/pubmed/17059896

10. Coumou H, Bel EH. Improving the diagnosis of eosinophilic asthma. Expert Rev Respir Med [Internet]. 2016 [cited 2019 Sep 23];10:1093-103. Available from: http://www.ncbi.nlm. nih.gov/pubmed/27624868

11. Bass DA. Behavior of eosinophil leukocytes in acute inflammation. I. Lack of dependence on adrenal function. J Clin Invest [Internet]. 1975 [cited 2019 Sep 23];55:1229-36. Available from: http://www.ncbi.nlm.nih.gov/pubmed/1133170

12. HILLS AG, FORSHAM PH, FINCH CA. Changes in circulating leukocytes induced by the administration of pituitary adrenocorticotrophic hormone in man. Blood [Internet]. 1948 [cited 2019 Sep 23];3:755-68. Available from: http://www.ncbi.nlm.nih.gov/pubmed/18869766

13. von Mutius E, Braun-Fahrländer C, Schierl R, Riedler J, Ehlermann S, Maisch S, et al. Exposure to endotoxin or other bacterial components might protect against the development of atopy. Clin Exp Allergy [Internet]. 2000 [cited 2018 Aug 1];30:1230-4. Available from: http:// www.ncbi.nlm.nih.gov/pubmed/10971468 
14. Zhao L, Bracken MB. Association of CD14 -260 (-159) C\&gt;T and asthma: a systematic review and meta-analysis. BMC Med Genet [Internet]. 2011 [cited 2018 Aug 1];12:93. Available from: http://www.ncbi.nlm.nih.gov/pubmed/21745379

15. Kamp VM, Leentjens J, Pillay J, Langereis JD, de Kleijn S, Kox M, et al. Modulation of granulocyte kinetics by GM-CSF/IFN- $\gamma$ in a human LPS rechallenge model. J Leukoc Biol [Internet]. 2013 [cited 2018 Jul 23];94:513-20. Available from: http://www.ncbi.nlm.nih. gov/pubmed/23794709

16. van Lier D, Geven C, Leijte GP, Pickkers P. Experimental human endotoxemia as a model of systemic inflammation. Biochimie [Internet]. 2018 [cited 2018 Jul 19]; Available from: https://www.sciencedirect.com/science/article/pii/S0300908418301718?via\%3Dihub

17. Hassani M, vanStaverenS, van Grinsven E, Bartels M, TesselaarK, Leijte G, etal. Characterization of the phenotype of human eosinophils and their progenitors in the bone marrow of healthy individuals. Haematologica [Internet]. 2019 [cited 2019 Sep 19]; haematol.2019.219048. Available from: http://www.ncbi.nlm.nih.gov/pubmed/31101758

18. Mori Y, Iwasaki H, Kohno K, Yoshimoto G, Kikushige $Y$, Okeda A, et al. Identification of the human eosinophil lineage-committed progenitor: revision of phenotypic definition of the human common myeloid progenitor. J Exp Med [Internet]. 2009;206:183-93. Available from: http://www.ncbi.nlm.nih.gov/pubmed/19114669

19. Konno M, Matsuda S, Kariyone S. [Eosinophil kinetics]. Nihon Rinsho [Internet]. 1993 [cited 2018 Feb 27];51:747-51. Available from: http://www.ncbi.nlm.nih.gov/pubmed/8492452

20. Farahi N, Singh NR, Heard S, Loutsios C, Summers C, Solanki CK, et al. Use of 111-Indiumlabeled autologous eosinophils to establish the in vivo kinetics of human eosinophils in healthy subjects. Blood [Internet]. 2012;120:4068-71. Available from: http://www.ncbi. nlm.nih.gov/pubmed/22993388

21. Berger $M$, de Boer JD, Bresser P, van der Poll T, Lutter R, Sterk PJ, et al. Lipopolysaccharide amplifies eosinophilic inflammation after segmental challenge with house dust mite in asthmatics. Allergy [Internet]. 2015 [cited 2018 Jul 27];70:257-64. Available from: http:// www.ncbi.nlm.nih.gov/pubmed/25381858

22. Nishihara F, Nakagome K, Kobayashi T, Noguchi T, Araki R, Uchida Y, et al. Trans-basement membrane migration of eosinophils induced by LPS-stimulated neutrophils from human peripheral blood in vitro. ERJ Open Res [Internet]. 2015 [cited 2018 Jul 27];1:00003-2015. Available from: http://www.ncbi.nlm.nih.gov/pubmed/27730145

23. Spijkerman R, Hesselink L, Hellebrekers P, Vrisekoop N, Hietbrink F, Leenen LPH, et al. Automated flow cytometry enables high performance point-of-care analysis of leukocyte phenotypes. J Immunol Methods [Internet]. 2019 [cited 2019 Dec 12];474. Available from: https://pubmed.ncbi.nlm.nih.gov/31419409-automated-flow-cytometry-enables-highperformance-point-of-care-analysis-of-leukocyte-phenotypes/

24. van der Meer W, Pickkers P, Scott CS, van der Hoeven JG, Gunnewiek JK. Hematological indices, inflammatory markers and neutrophil CD64 expression: comparative trends during experimental human endotoxemia. J Endotoxin Res [Internet]. 2007 [cited 2018 Oct 19];13:94-100. Available from: http://www.ncbi.nlm.nih.gov/pubmed/17621550

25. Horie S, Gleich GJ, Kita H. Cytokines directly induce degranulation and superoxide production from human eosinophils. J Allergy Clin Immunol [Internet]. 1996 [cited 2018 Sep 26];98:371-81. Available from: http://www.ncbi.nlm.nih.gov/pubmed/8757214

26. Johansson MW. Activation states of blood eosinophils in asthma. Clin Exp Allergy [Internet]. 2014 [cited 2019 Oct 2];44:482-98. Available from: http://doi.wiley.com/10.1111/cea.12292 
27. Dobrina A, Menegazzi R, Carlos TM, Nardon E, Cramer R, Zacchi T, et al. Mechanisms of eosinophil adherence to cultured vascular endothelial cells. Eosinophils bind to the cytokineinduced ligand vascular cell adhesion molecule- 1 via the very late activation antigen-4 integrin receptor. J Clin Invest [Internet]. 1991 [cited 2018 Jul 27];88:20-6. Available from: http://www.ncbi.nlm.nih.gov/pubmed/1711540

28. Jia GQ, Gonzalo JA, Hidalgo A, Wagner D, Cybulsky M, Gutierrez-Ramos JC. Selective eosinophil transendothelial migration triggered by eotaxin via modulation of Mac-1/ICAM1 and VLA-4/VCAM-1 interactions. Int Immunol [Internet]. 1999 [cited 2018 Sep 25];11:110. Available from: http://www.ncbi.nlm.nih.gov/pubmed/10050668

29. Malhotra R, Priest R, Bird MI. Role for L-selectin in lipopolysaccharide-induced activation of neutrophils [Internet]. Vol. 593, Biochem. J. 1996 [cited 2018 Oct 5]. p. 589-93. Available from: https://www.ncbi.nlm.nih.gov/pubmed/8973571

30. Mishra A, Hogan SP, Lee JJ, Foster PS, Rothenberg ME. Fundamental signals that regulate eosinophil homing to the gastrointestinal tract. J Clin Invest [Internet]. 1999 [cited 2018 Oct 5];103:1719-27. Available from: http://www.ncbi.nlm.nih.gov/pubmed/10377178

31. Robertson SA, Mau VJ, Young IG, Matthaei KI. Uterine eosinophils and reproductive performance in interleukin 5-deficient mice. J Reprod Fertil [Internet]. 2000 [cited 2018 Oct 5];120:423-32. Available from: http://www.ncbi.nlm.nih.gov/pubmed/11058459 


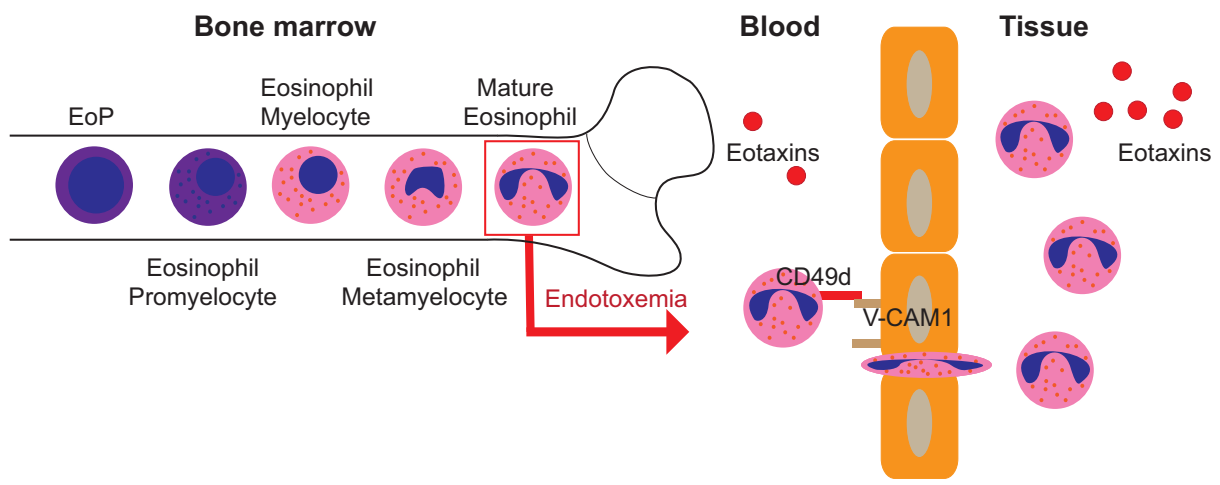

\section{Graphical abstract}

\begin{tabular}{|l|l|l|l|}
\hline Antibody & Fluorochrome & Clone & Company \\
\hline IL-5R $\alpha$ & BV421 & A14 & BD \\
\hline CD66b & PerCP-Cy5.5 & G10F5 & BD \\
\hline CD64 & APC & 10.1 & Biolegend \\
\hline CD193 & BV510 & $5 E 8$ & Biolegend \\
\hline CD11b & AF700 & CBRM 1/5 & Biolegend \\
\hline CD62L & BV650 & DREG-56 & Biolegend \\
\hline CD35 & FITC & E11 & Biolegend \\
\hline CD11b & APC-AF750 & Bear1 & Beckman Coulter \\
\hline CD16 & BV785 & 3G8 & Sony Biotechnology \\
\hline CD49d & PE-Cy7 & 9F10 & Sony Biotechnology \\
\hline
\end{tabular}

Supplementary Table 1. Overview of used antibodies for staining 
$\mathbf{A}$
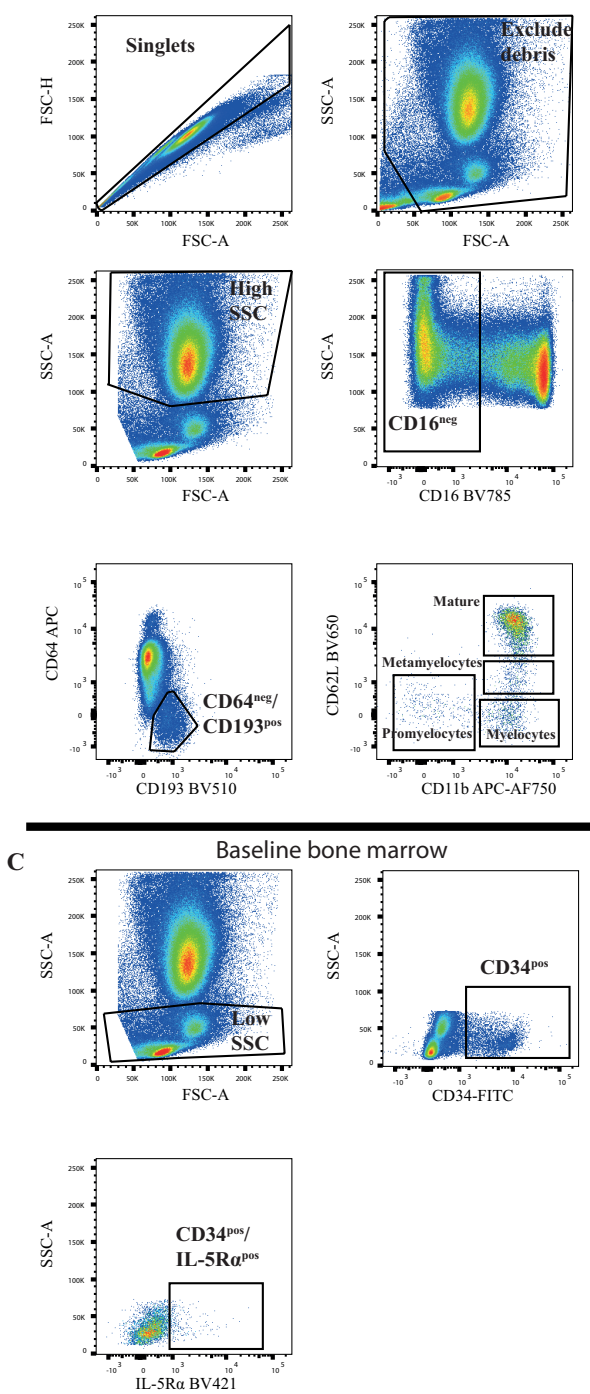

B

4 hours after endotoxin bone marrow
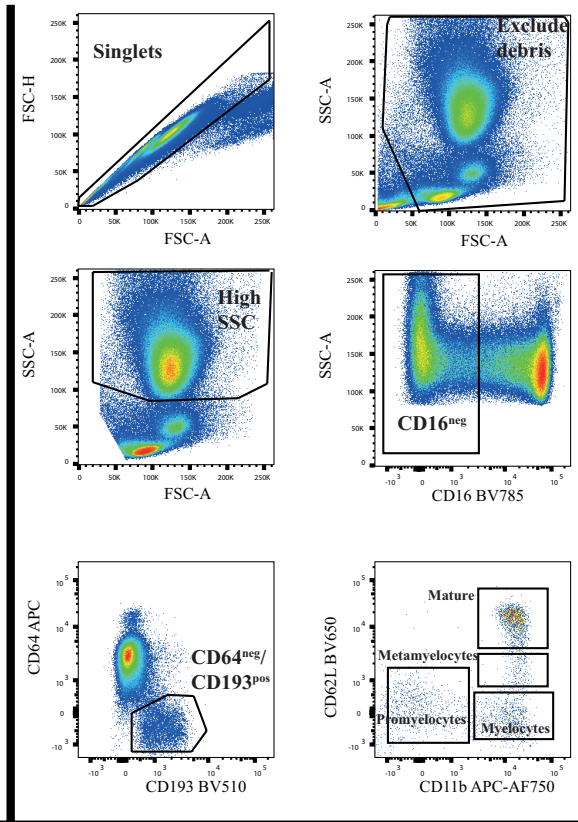

D

4 hours after endotoxin bone marrow

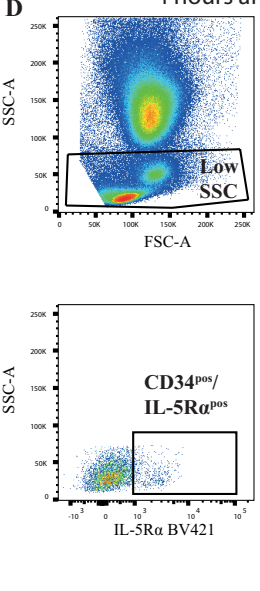

\section{Supplementary Figure 1. Gating strategy for eosinophils and their progenitors in BM and blood.}

A. Eosinophils and their progenitors in the bone marrow at baseline were identified as follows: first singlets were gated using FSC-H and FSC-A, secondly debris was excluded using SSC-A and FSC-A, thirdly only cells with a high SSC-A cells were gated, fourthly CD16 $6^{\text {neg }}$ cells were gated, fifthly eosinophils and their progenitors were gated as $\mathrm{CD} 193^{\text {pos }}$ and $\mathrm{CD} 64^{\text {neg }}$ and finally eosinophil promyelocytes were gated as $C D 11 b^{\text {neg }}$ and $C D 62 L^{\text {neg }}$, eosinophil myleocytes were gated as $C D 11 b^{\text {pos }}$ and $C D 62 L^{\text {neg }}$, eosinophil metamyelocytes were gated as $\mathrm{CD} 11 \mathrm{~b}^{\text {pos }}$ and $\mathrm{CD} 62 \mathrm{~L}^{\mathrm{dim}}$ and mature eosinophils were gated as $\mathrm{CD} 11 \mathrm{~b}^{\text {pos }}$ and CD62 $L^{\text {high }}$. B. eosinophils and their progenitors were similarly identified after endotoxin administration. 
C. Early lineage commited eosinophil progenitors (EoPs) were gated as follows: first only low SSC cells were gated, secondly $\mathrm{CD} 34^{\text {pos }}$ cells were gated and finally CD34 $4^{\text {pos }}$ and IL-5R $\alpha^{\text {pos }}$. D. EoPs were similarly identified after LPS administration.

A

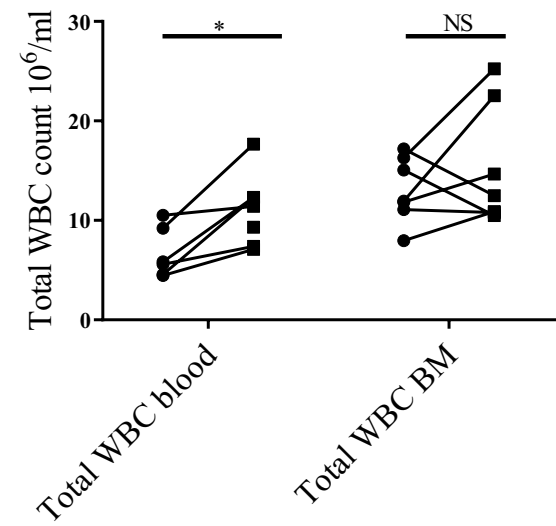

B

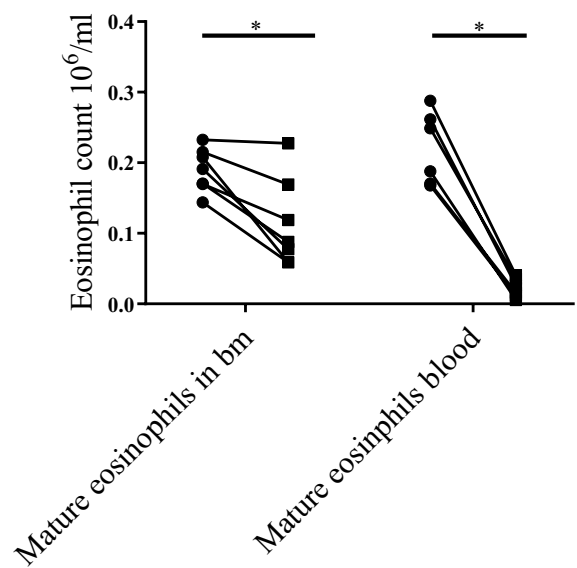

Supplementary Figure 2. Change in absolute numbers of white blood cells and mature eosinophils in the blood and bone marrow before and after LPS administration.

A. Total white blood cell counts $\left(10^{6}\right.$ cells $\left./ \mathrm{ml}\right)$ are depicted before and after LPS. B. Abolute number of mature eosinophils $\left(10^{6} \mathrm{cells} / \mathrm{ml}\right)$ are depicted before and after LPS. A Wilcoxon matched-pairs signed rank test was performed. NS: not significant. ${ }^{*} \mathrm{P} \leq 0.05$ 
A

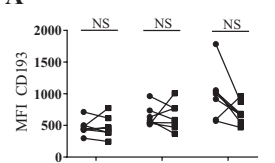

D

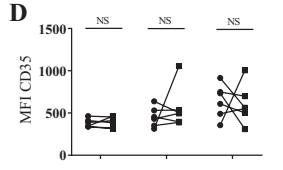

G

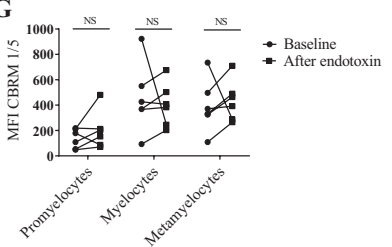

B

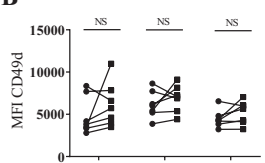

E
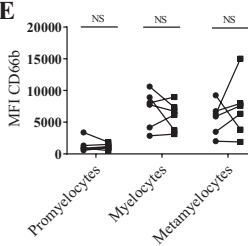

C

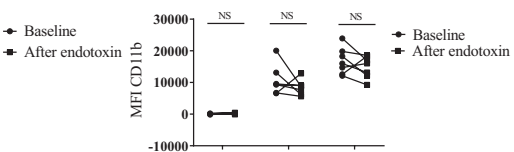

F
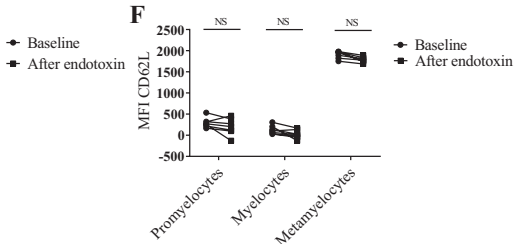

Supplementary Figure 3. Change of surface markers on immature eosinophils in the BM before and after LPS administration.

Median fluorescence intensity is plotted for the different surface markers of immature eosinophils in bone marrow (from promyelocyte stage until metamyleocyte stage) at baseline and 4 hours after endotoxin administration. A.CD193. B.CD49d. C. CD11b. D. CD35 E. CD66b, F. CD62L and G. CBRM 1/5 (antibody that only recognizes the activated epitope of Mac-1). A Wilcoxon matched-pairs signed rank test was performed. NS: not significant.

$\mathbf{A}$

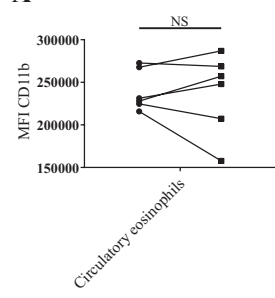

B

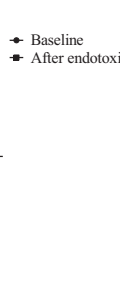

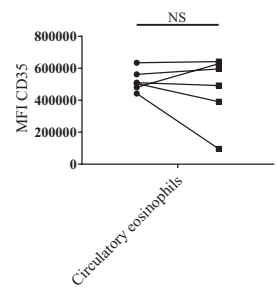

C

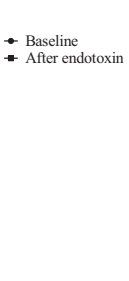

Supplementary Figure 4. Change of surface markers on mature eosinophils in the BM and blood before and after LPS administration measured with a fully automated flow cytometer.

Median fluorescence intensity is plotted for the different surface markers of mature eosinophils in the blood at baseline and after endotoxin administration $(n=6)$. Samples were measured as fast as possible after blood withdrawal on a fully automated flow cytometer (Aquios of Beckman Coulter). A. CD11b, B. CD35 and C. CD62L. A Wilcoxon matched-pairs signed rank test was performed. NS: not significant. 


\title{
Chapter 9
}

\section{The effect of mepolizumab on eosinophil and basophil kinetics in patients with eosinophilic asthma}

\author{
M. Hassani ${ }^{1,2}$, T. Tak ${ }^{1,2}$ C. van Aalst ${ }^{1,2}$, S. Van Nederveen ${ }^{3}$, K. Tesselaar ${ }^{1}$, J.A.M.
} Borghans $^{1}$, N. Vrisekoop ${ }^{1,2}$ and L. Koenderman ${ }^{1,2}$.

1. Center for Translational Immunology, University Medical Center Utrecht, Utrecht, The Netherlands.

2. Department of Respiratory Medicine, University Medical Center Utrecht, Utrecht, The Netherlands.

3. Department of Respiratory Medicine, HAGA hospital, The Hague, The Netherlands. 


\section{Abstract}

Eosinophils play an essential role in the pathogenesis of asthma. However, little is known about the kinetics of these cells in blood and sputum.

In this study the kinetics of eosinophils and basophils were determined in blood and in sputum of patients with moderate to severe eosinophilic asthma who were treated with mepolizumab $(n=10)$ or placebo $(n=10)$. The kinetics of these cells was studied after short term treatment ( 4 days; $1^{\text {st }}$ phase) and long-term treatment ( 84 days; $2^{\text {nd }}$ phase) of treatment with mepolizumab and placebo by metabolic labelling patients twice with $6,6-{ }^{2} \mathrm{H}_{2}$-glucose (deuteriated glucose) for 6 hours. After labelling, blood and sputum was collected at different time points and eosinophils and basophils were isolated by FACS sorting. DNA ${ }^{2} \mathrm{H}$-enrichment was determined by GC-MS.

During the $1^{\text {st }}$ phase loss of label in the DNA of blood eosinophils was significantly delayed $(p<0.001)$ in the mepolizumab group compared to placebo despite a rapid decline of the absolute eosinophil count in the blood. On the other hand, loss of label in sputum eosinophils was faster in the mepolizumab group. In marked contrast, after 84 days of treatment with mepolizumab or placebo the remaining blood eosinophils showed highly comparable kinetics. Loss of label in DNA of sputum eosinophils was still faster after longer treatment with mepolizumab. Kinetics of blood basophils were largely similar at the $1^{\text {st }}$ and $2^{\text {nd }}$ phase of this study. Also, the number of basophils was not affected by treatment with anti-IL-5.

Our findings in eosinophils implicate that short-term treatment with mepolizumab leads to a decrease of homing or a slower turnover of (a certain population of) eosinophils in the bone marrow. The long-term treatment with mepolizumab implicates that similar to IL-5 knock-out mice, human eosinophil differentiation is not IL-5 dependent. Apparently, IL-5 seems more a growth factor of IL-5R expressing eosinophil progenitors rather than a differentiation factor. Data of the basophil kinetics suggests that their survival and proliferation are not dependent on IL-5. 


\section{Introduction}

Asthma is a chronic respiratory condition characterized by bronchial inflammation leading to reversible airways obstruction and airway hyper responsiveness. The disease is characterized by episodes of (progressive) increase in shortness of breath, cough, wheezing and chest tightness ${ }^{1}$. It is a very common disorder affecting around 300 million individuals of all ages around the world ${ }^{2}$. This makes asthma a major cause of morbidity and utilization of health resources ${ }^{3}$.

Nowadays, asthma is no longer seen as a single entity but rather as a disease with multiple endotypes ${ }^{4}$. It can for example be classified as atopic or non-atopic, based on the presence or absence of specific IgE to inhaled allergens, respectively ${ }^{5}$. Another possible way to classify asthma is based on the absence or presence of eosinophilic inflammation. Eosinophilic asthma is characterized by the presence of a significant number of sputum and/or blood eosinophils, whereas non-eosinophilic asthma is characterized by either the presence of high numbers of sputum neutrophils (neutrophilic) or very few inflammatory sputum cells (paucigranulocytic) ${ }^{6}$. The need for classifying asthma has gained importance with the development of novel and specific molecular therapies that are only efficacious in particular subgroups of asthma ${ }^{4}$. This is particularly true for eosinophilic asthma, since IL-5 targeted therapy proved to be only efficacious in asthma characterized by eosinophilic inflammation ${ }^{7}$. Mepolizumab and reslizumab are both humanized monoclonal antibodies (Mab) that bind and neutralize circulating IL-5 ${ }^{8}$. Benralizumab on the other hand is a Mab that binds directly to the IL-5 receptor which leads to the induction of cell death by antibody-dependent cellular cytotoxicity $(\mathrm{ADCC})^{9}$

In humans the effects of IL-5 are restricted to basophils and eosinophils ${ }^{10}$ although a preliminary study suggest the presence of IL5-R on ILC2' ${ }^{11}$. The precise functions of IL-5 in humans have not been completely identified, because most studies have either been performed in vitro or in animal models ${ }^{8}$. Murine studies suggest that IL-5 is important in the induction of reactive eosinophilia during allergen provocations and parasitic infections ${ }^{12}$. The role of IL-5 in homeostatic eosinopoiesis is much less clear as IL-5 knockout mice and mice with deleted $\beta$-common and $\beta$-IL-3 genes do have eosinophils ${ }^{13,14}$. Apparently, IL-5 seems more a growth factor of IL-5R expressing eosinophil/basophil progenitors rather than a differentiation factor.

Treating asthma patients with mepolizumab leads to a fast reduction in the numbers of eosinophils and their progenitors in blood and the bone marrow but their numbers do not go to zero ${ }^{15}$. This suggests that targeting IL-5 blocks the proliferation of eosinophils in humans. However, treating asthma patients with a high dose of mepolizumab only reduced $50 \%$ of the eosinophil numbers in bronchial biopsies even after 10 weeks of treatment ${ }^{16}$. A good explanation for this finding has not been provided yet. For example, it is unknown whether eosinophils after anti-IL-5 are long lived in the tissue or that the little number of eosinophils produced (independently of IL-5) preferentially home to the bronchial airways. Moreover, studies in which the life cycle of eosinophils has been studied are rare. 
The life cycle refers to the phases of 1 . proliferation and differentiation in the bone marrow, 2 . half-life in the peripheral blood, 3 . the time that the cells stay in and exit from the tissues and 4 . the moment of programmed cell death. The consensus in literature is that eosinophils and basophils are very short lived (half-life of 2-3 days and 3-4 days in peripheral blood, respectively) ${ }^{17,18}$. They are continuously produced by the bone marrow and die by apoptosis in the tissue ${ }^{19}$. These insights in humans are based on ex vivo labelling of cells with (radioactive) tracers before reinfusion into the body or by toxic tracers in vivo ${ }^{20,21,22}$. However, this methodology is flawed by the fact that in vitro manipulation activates leukocytes and significantly affects the homing behavior of cells, thereby leading to underestimation of the half-lives of non-touched cells ${ }^{23}$.

In this study we used deuterated glucose to label 20 patients with moderate to severe asthma in vivo in order to study the life cycle of eosinophils and basophils in the blood and sputum (eosinophils only). Additionally, these patients were either treated with placebo $(n=10)$ or mepolizumab $(n=10)$. This the first report that reliably determined eosinophil kinetics in humans, because ${ }^{2} \mathrm{H}$ labelling allowed labelling of cells without toxic effects ${ }^{24}$.

\section{Materials and methods}

\section{Subjects}

A total of 20 patients with moderate to severe eosinophilic asthma (EA) were recruited at the respiratory medicine department of the University Medical Center in Utrecht and of the Haga Hospital in the Hague (ABR NL57535.041.16). All participants gave a written informed consent in accordance to the Declaration of Helsinki (Forteleza, 2013). The study was approved by the local institutional review board (IRB).

All participants were between the age of 18-70 years and were diagnosed with asthma according to GINA guidelines by an experienced respiratory physician. In short: patients had clinical features (episodic shortness of breath, often accompanied by cough and/or wheezing) and reversibility of the forced expiratory volume in 1s (FEV1) after treatment with $400 \mu \mathrm{g}$ of salbutamol ( $\geq 12 \%$ or $\geq 200 \mathrm{ml}$ compared to baseline). The eosinophilic phenotype of asthma was defined as at least one blood test within one year before enrollment with a minimal blood eosinophil concentration of $0.25 \times 10^{6} / \mathrm{ml}$. Patients with auto-immune diseases (e.g. rheumatoid arthritis), insulin-dependent diabetes, cancer and chronic infection were excluded. Smokers and patients with proven allergic bronchopulmonary aspergillosis (ABPA) were also excluded.

Because of lack of data from sputum eosinophils, we added data from an earlier study in which 9 patients with eosinophilic asthma were enrolled (EHLA study, ABR NL44779.041.13). These patients had similar inclusion and exclusion criteria to this study (table 1), but were not randomized for treatment with mepolizumab or placebo. They were kept on their usual treatment management. 


\begin{tabular}{|l|l|l|l|}
\hline Patient characteristics & Placebo & Mepolizumab & EHLA \\
\hline $\mathrm{N}$ & 10 & 10 & 9 \\
\hline Female (\%) & $5(50 \%)$ & $6(60 \%)$ & $4(44 \%)$ \\
\hline Age at inclusion, years (IQR) & $51(33-65)$ & $57(46-62)$ & $56(40-72)$ \\
\hline BMI, kg/m² (IQR) & $26.2(23.5-34.0)$ & $28.9(24.6-35.1)$ & $27.2(23.2-32.2)$ \\
\hline FEV1, L (IQR) & $2.51(1.92-3.79)$ & $2.33(1.57-3.24)$ & $2.45(1.47-3.10)$ \\
\hline FEV1,\% predicted (IQR) & $88.5(70.8-101.0)$ & $64.0(60.1-84.3)$ & $73.7(52.2-96.6)$ \\
\hline ACQ score at inclusion (IQR) & $1.9(1.3-2.3)$ & $2.2(1.6-2.8)$ & $1.53(0.14-2.57)$ \\
\hline ACQ score after 80 days (IQR) & $1.6(1.3-3.3)$ & $2.0(1.1-2.8)$ & N/A \\
\hline $\begin{array}{l}\text { Blood eosinophil count at } \\
\text { inclusion 10\%/ml, (IQR) }\end{array}$ & $0.49(0.28-0.80)$ & $0.40(0.22-0.70)$ & $0.54(0.20-2.2)$ \\
\hline $\begin{array}{l}\text { Blood basophil count at } \\
\text { inclusion 10\%/ml, (IQR) }\end{array}$ & $0.035(0.027-.070)$ & $0.046(0.023-0.071)$ & N/A \\
\hline \begin{tabular}{l} 
Medication score (IQR)\# \\
\hline
\end{tabular} & $4(4-4)$ & $4(3-4)$ & 4 (3-5) \\
\hline
\end{tabular}

Table 1. Patient characteristics in the placebo and mepolizumab group, including the characteristics of an earlier eosinophilic asthma study (EHLA).

Data represent median values (interquartile range). \# 5-point ordinal scale based on guidelines of the British Thoracic Society. $0=$ no medication, $1=$ inhaled SABA when required, $2=$ low dose ICS and SABA, $3=$ medium dose ICS + LABA, $4=$ high dose of ICS+LABA and $5=$ high dose of ICS (and LABA) with OCS.

\section{Deuterium labelling procedure and sampling}

All participants were in vivo labelled with deuterium-labelled glucose $\left({ }^{2} \mathrm{H}\right)$ as was published previously ${ }^{24}$. In short, 12 doses of metabolic grade $6,6-{ }^{2} \mathrm{H}_{2}$-glucose amounting to a total of $1 \mathrm{~g} / \mathrm{kg}$ bodyweight (Cambridge Isotope Laboratories, Tewksbury, MA, USA) were administered orally, every half hour. In vivo, $6,6-{ }^{2} \mathrm{H}_{2}$-glucose is metabolized and incorporated into the DNA of only dividing cells via the de novo nucleotide synthesis pathway ${ }^{25}$. During the labelling procedure, three small blood samples were obtained via finger pricks on filter papers to determine the availability of label in plasma (plasma glucose enrichment ${ }^{25}$. The labelling procedure was performed twice per patient: at the start of the study ( $t=0$ days, phase 1 ) and after 80 days ( $t=0$ days, phase 2$)$, see Figure 1. For all participants, a total of 10 blood samples were obtained, 2 prior to both labelling procedures and 8 other time points (Figure 1). In addition, three sputum samples per patient were obtained. For blood and sputum, the time points varied between volunteers, but were largely similar between the mepolizumab and placebo group to maximize the total number of data points for each group. 
A

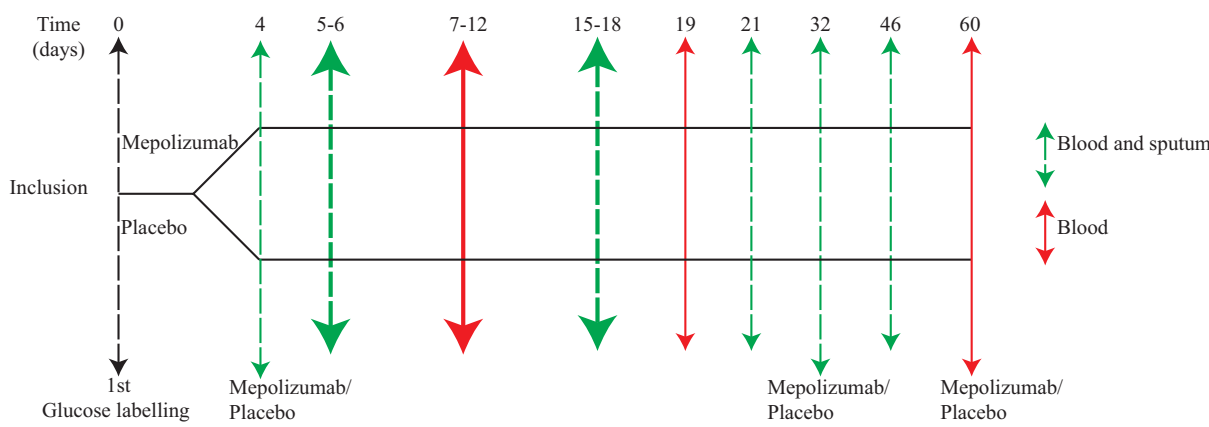

2nd phase

B

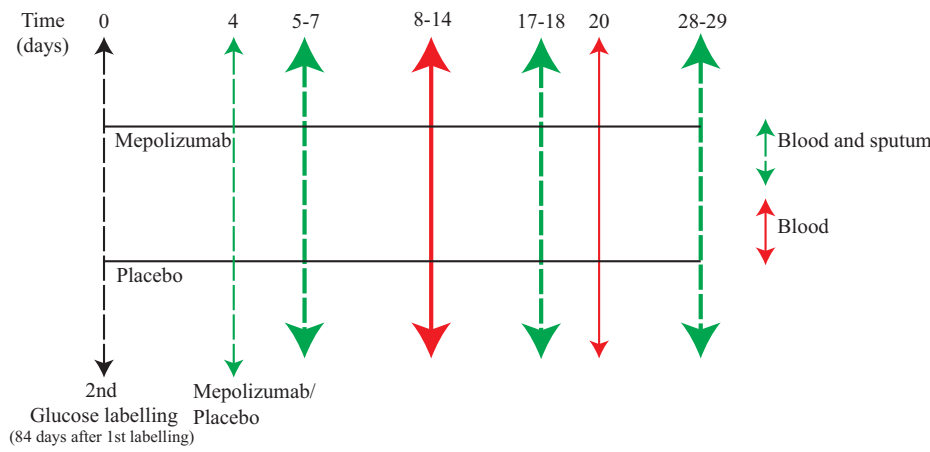

Figure 1. Flowchart of study design.

A. During the $1^{\text {st }}$ phase of the study 20 asthma patients were enrolled. At day 0 , all patients were labelled with deuterium glucose and baseline blood sample was withdrawn. Thereafter patients were blindly randomized for the mepolizumab $(n=10)$ or placebo group $(n=10)$. In both groups only blood (red arrows) or blood and sputum (green arrows) samples were taken on different days, starting from day 4 after label intake. $1^{\text {st }}$ phase ended at day 60 after label intake. Mepolizumab or placebo was administered for the $1^{\text {st }}$ time at day 4 after label intake. This was followed by a $2^{\text {nd }}$ and $3^{\text {rd }}$ administration of mepolizumab or placebo, each time 28 days later (at day 32 or 60 respectively). B. $2^{\text {nd }}$ phase started at 84 days after the $1^{\text {st }}$ label intake. At this day all patients were again labelled with deuterium glucose and blood was withdrawn. 4 days after the $2^{\text {nd }}$ label intake, the $4^{\text {th }}$ and last mepolizumab or placebo dose was administered. Again, either blood only (red arrows) or blood and sputum (green arrows) were taken on several following days. 


\section{Randomization of subjects and drug administration}

After enrollment and the first labelling procedure patients were blindly randomized for placebo $(n=10)$ or $100 \mathrm{mg}$ mepolizumab $(n=10)$. Both injections were administered every 4 weeks ( 28 days) subcutaneously in the upper arm for a total of 4 times (Figure 1 ). The first administration of mepolizumab or placebo was 4 days after deuterium labelling. For baseline characteristics of both groups, see Table 1.

\section{Blood collection and processing}

At each time point, $18 \mathrm{ml}$ of blood was collected in sodium heparin tubes (Vacuette ${ }^{\circledR}$ Greiner bio-one, Kremsmünster, Austria). Subsequently, erythrocytes were lysed using an ice-cold lysis buffer (150 $\mathrm{mM} \mathrm{NH}_{4} \mathrm{Cl}, 10 \mathrm{mM} \mathrm{KHCO}_{3}$ and $0.1 \mathrm{mM} \mathrm{NA}_{2}$ EDTA). After this, the remaining leukocytes were washed and resuspended in a staining buffer (PBS2+) consisting of PBS supplemented with $0.32 \% \mathrm{w} / \mathrm{v}$ trisodium citrate (prepared by the pharmacy of the University Medical Centre Utrecht) and 10\% w/v human plasma albumin solution (Sanquin, Amsterdam, the Netherlands).

\section{Sputum induction and processing}

Sputum was induced as described before ${ }^{26}$. In short, induction of sputum was accomplished by inhalation of 3-5\% saline aerosols. Samples were thereafter incubated with 4:1 (w/vol) 0.1\% sputolysin solution in PBS with a corrected osmolarity of 280-290 mOsm (Merck Millipore, Darmstadt, Germany) in a $37^{\circ} \mathrm{C}$ waterbath for 20 minutes ${ }^{26}$. The resulting cell suspensions were then filtered through a $40 \mu \mathrm{m}$ filter (Corning, New York, USA) and washed with PBS. For all sputum samples cytospin slides were obtained and stained with May-Grünwald-Giemsa. The remaining sample was used for granulocyte isolation as is described below.

\section{Granulocyte isolation from sputum and blood}

In PBS suspended sputum samples were stained with a live/dead violet cell staining kit (Thermo Fisher Scientific, Waltham, MA, USA). After washing, cells were suspended in PBS2+ and stained with the following antibodies: from Biolegend (San Diego, CA, USA): CD16-PE/Cy7 (clone 3g8), CD34-Alexa Fluor 700 (clone 581), CD206-PerCP/Cy5.5 (clone 15-2), and IL-3R $\alpha$-Brilliant Violet 605 (clone 6H6); from BD Biosciences (San Jose, CA, USA): CD14 APC-H7 (clone MPHIP9); from Beckman Coulter (Pasadena, CA, USA): CD45-Krome Orange (clone J33), from Thermo Fisher Scientific (Waltham, MA, USA): IL-5R $\alpha-P E$ (clone 26815) and CD9-Cy2 (clone S32 a kind gift from ing. D. Kanters). Lysed blood was similarly stained but without the live dead stain, CD45 and CD206. In addition, CD193-Alexa Fluor 647 (clone 5E8) and CD62L-PerCP/Cy5.5 (clone DREG-56), both from Biolegend (San Diego, CA, USA) were only used to stain blood samples.

Cells were isolated using an Ariall or ARIAIII FACS sorter (BD Biosciences, Mountain view, CA, USA). Blood granulocytes were identified based on doublet exclusion, FSC/ SSC properties and CD14- (Supplementary Figure 1 for gating strategy) ${ }^{27}$. Subsequently, neutrophils were identified as CD16 high and CD62 $\mathrm{L}^{\text {high }}$ and eosinophils as $\mathrm{CD}^{-} 6^{-}$and $\mathrm{CD}_{193^{+}}$and $\mathrm{CD} 9^{+}$. Sputum cells were gated similarly, with addition of a Live/DEAD stain and CD45 stain to exclude necrotic cells and non-leukocytes (Supplementary Figure 2). Sorted populations were re-analyzed (typically $>99 \%$ pure). Cellular DNA was isolated from fresh samples using a NucleoSpin Blood kit according to producers' instructions 
(Machery-Nägel, Oensingen, Switzerland). DNA was stored in a $-20^{\circ} \mathrm{C}$ until further processing.

\section{Determination of DNA ${ }^{2} \mathrm{H}$ enrichment}

After collection of all samples, DNA was enzymatically hydrolyzed into deoxy ribonucleotides and derivatized to pentafluoro triacetate derivates (PFTA) as described previously ${ }^{28}$. Thereafter the quantity of ${ }^{2} \mathrm{H}$-labelled and unlabelled adenosine derivatives was measured on an Agilent $7980 \mathrm{~A} / 5975 \mathrm{C}$ GC-MS in negative chemical ionization mode scanning for $\mathrm{m} / \mathrm{z} 435(\mathrm{M}+0$, unlabelled) and $\mathrm{m} / \mathrm{z} 437(\mathrm{M}+2$, labelled). The resulting enrichments were corrected for natural background enrichment, availability of $6,6-{ }^{2} \mathrm{H}_{2}$-glucose in plasma and the ratio adenosine that is de novo synthesized/obtained by base salvage (0.65) as published previously ${ }^{25}$.

\section{Data analysis}

FlowJo v10 (LLC, Ashland, OR, USA) was used to analyze the flow cytometry data. Statistical analysis was performed using GraphPad Prism 8.0 (Graphpad Software, La Jolla, CA, USA).

\section{Results}

\section{Granulocyte numbers in blood and sputum.}

The mepolizumab and placebo group ( $n=10$ for both groups) were comparable for all tested baseline parameters (see table 1). The absolute count of blood eosinophils showed a rapid and steady decline in the mepolizumab group (Figure $2 \mathrm{~A}$ ) and blood eosinophils were down within 2 days of treatment. Basophil numbers in the circulation did not show a significant difference in numbers between the mepolizumab and placebo group (Figure 2B). In the sputum, the percentage of eosinophils also declined within 42 days (Figure 2C). Interestingly, the number of eosinophil progenitors (EoPs) did not show any significant alterations between the two groups in blood or sputum (Supplementary Figure 3). Basophil numbers in sputum were very low and, therefore, unreliable to enumerate (data not shown).

\section{Phenotyping granulocytes}

Since the number of eosinophils in blood and sputum clearly decreased after mepolizumab we tested the hypothesis that the remaining eosinophils would be phenotypically different. So we compared several membrane receptor expression on blood eosinophils. The expression of IL-5R $\alpha$ and IL-3R $\alpha$ did not change significantly in both eosinophils and basophils (Figure 3A-D). However, when we compared multiple paired samples after mepolizumab and placebo, there was a significant decrease of IL-3R $\alpha$ expression in blood eosinophils (3F) after treatment with mepolizumab. There was no difference in IL-5R $\alpha$ expression in blood and sputum eosinophils after treatment with mepolizumab (Figure 3EF). IL-5R $\alpha$ expression and IL-3R $\alpha$ expression were lower and higher in sputum eosinophils when compared to blood eosinophils, respectively. We also determined the expression of CD62L and CD193 after treatment with mepolizumab or placebo (Supplementary Figure 4). Interestingly CD62L increased after treatment with mepolizumab (Supplementary Figure 4A), whereas CD193 did not change (Supplementary Figure 4B). 


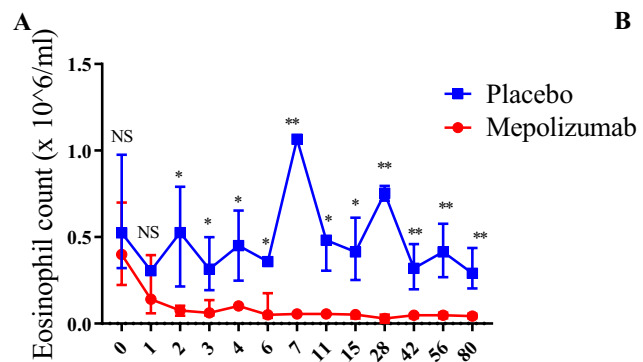

Days after placebo/mepolizumab

C

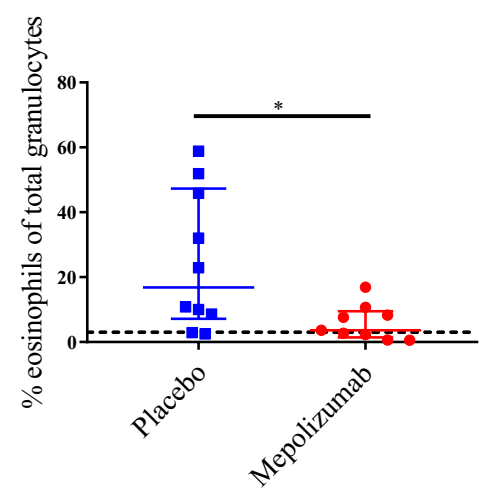

B

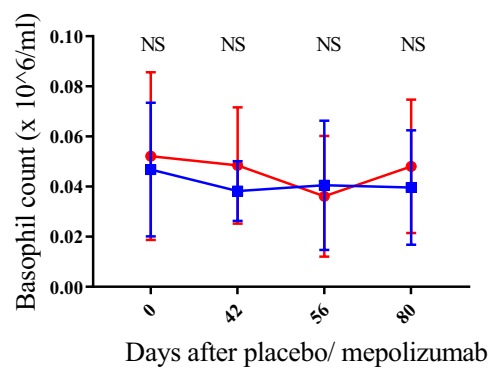

Figure 2. Change of eosinophil and basophil numbers in blood and sputum.

A. The absolute number of blood eosinophils was determined before and several days after administration of mepolizumab (red) or placebo (blue). B. Similarly the absolute number of blood basophils was determined before and several days after administration of mepolizumab (red) or placebo (blue). C. From 10 patients in the placebo group and 9 patients in the mepolizumab group the percentage of eosinophils of total granulocytes in the sputum was determined after at least 42 days after mepolizumab (red) or placebo (blue). Median with interquartile range is indicated in each graph. A Mann Whitney test was performed to compare placebo with mepolizumab at each time point. NS not significant; ${ }^{*} \mathrm{P} \leq 0.05$ and $* * \mathrm{P} \leq 0.01$. 
A

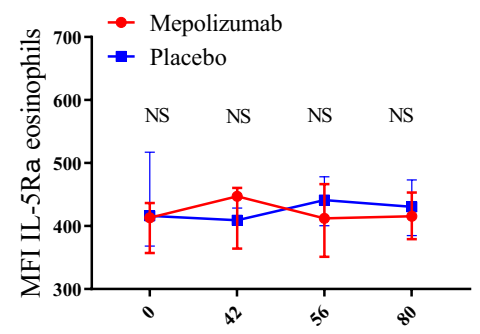

Days after placebo/mepolizumab
B

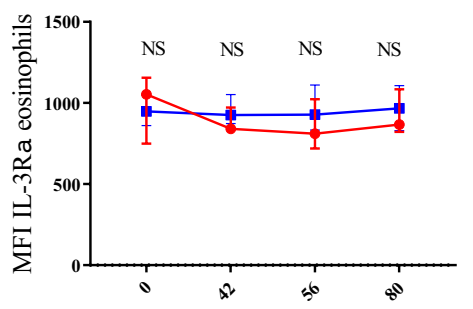

Days after placebo/ mepolizumab
C

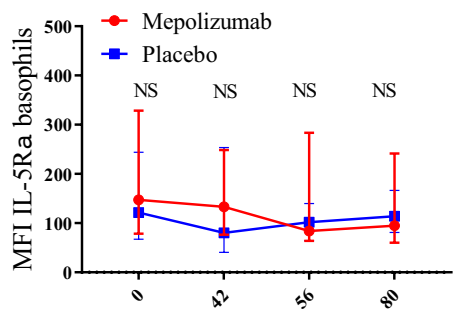

Days after placebo/ mepolizumab
E

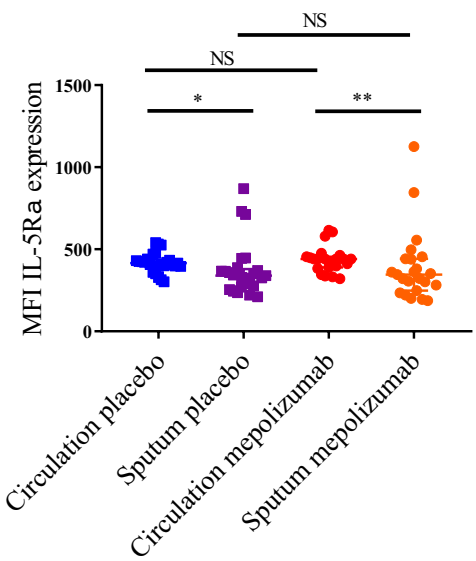

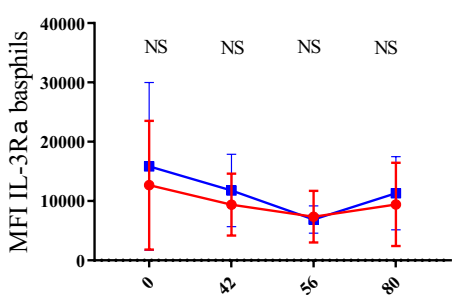

Days after placebo/mepolizumab
D

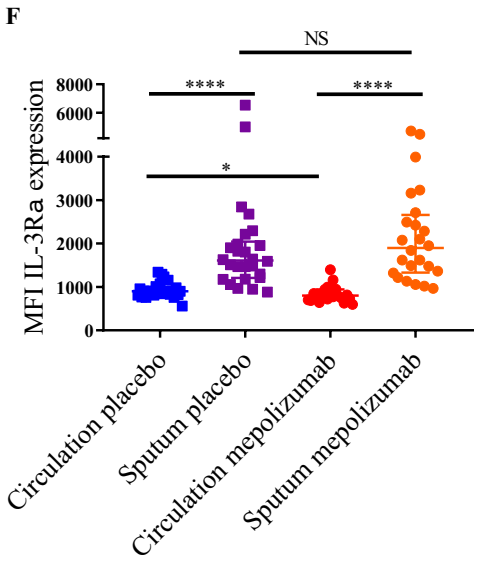

Figure 3. Change of eosinophil and basophil receptor expression in blood and sputum.

The median fluorescence (MFI) intensity on the cell membrane is plotted for IL-5R $\alpha(\mathbf{A})$ and IL-3R $\alpha(B)$ in blood eosinophils at different time points after either placebo (blue) or mepolizumab (red) administration. This was similarly done for blood basophil expression of IL-5R $\alpha$ (C) and IL-3R $\alpha$ (D). E. MFI of IL-5R $\alpha$ expression was also determined for multiple samples after at least 3 days of treatment with mepolizumab or placebo in blood (red and blue respectively) and sputum (purple and orange respectively). F. This was similarly done for the MFI of IL-3R $\alpha$ expression. Median with interquartile range is indicated in each graph. A Mann Whitney test was performed to compare placebo with mepolizumab and a Wilcoxon test was done to compare blood with sputum. NS not significant, $* \mathrm{P} \leq 0.05 ; * \mathrm{P} \leq 0.01$ and $* * * * \mathrm{P} \leq 0.0001$. 


\section{Eosinophil kinetics in blood}

10 patients in the placebo group and 10 patients in the mepolizumab group were sampled at multiple days. Individual patients within each group were sampled on different days in order to have as much as possible information about eosinophil kinetics in time. The chosen time points were largely similar between the two groups. Moreover, all patients underwent two separate labelling procedures: a first one at baseline (day $0 ; 1^{\text {st }}$ phase) and a second one after 84 days (12 weeks) after first labelling procedure ( $2^{\text {nd }}$ phase). During the course of the study, mepolizumab or placebo was administered every 4 weeks (28 days) with the first administration at day 4 after the first label intake (Figure 1). During the first uplabelling phase (from day 0 to day 7 after labelling intake, Figure 4C), the increase of ${ }^{2} \mathrm{H}$-label originating from eosinophil DNA is similar for both placebo and mepolizumab treatment. From day 8 on the loss of ${ }^{2} \mathrm{H}$-label is slower in the mepolizumab group compared to the placebo group (linear regression, $\mathrm{P}=0.0013$ ). Samples measured from day 32 and beyond were unlabelled in both groups.

Interestingly, the difference in down-labelling measured between placebo and mepolizumab during the first phase, were not found during the second phase (Figure $4 D, P=0.18$ ). So, after 12 weeks of treatment with mepolizumab or placebo remaining eosinophils show similar kinetical behavior.

\section{Eosinophil kinetics in sputum}

Sputum samples were either taken close to or far from the day of label intake, because it was hypothesized that ${ }^{2} \mathrm{H}$ enrichment in eosinophils would be different on those days after treatment with anti-IL-5 (see Discussion). Indeed, when sputum eosinophil enrichment was compared between the placebo and mepolizumab group during the $1^{\text {st }}$ phase, the mepolizumab group showed lower ${ }^{2} \mathrm{H}$-enrichment on day 14 and beyond (Figure $5 \mathrm{~A}$, simple linear regression, $\mathrm{P}=0.0058$ ). Similar to blood, deuterium label on days close to the day of label intake (day 4-6) were not different between the two groups $(P=0.93)$. During the $2^{\text {nd }}$ phase of the study, again the first days showed a very similar pattern $(P=0.93)$, but loss of label seemed to be even more rapidly in the mepolizumab group (Figure 5B, $P=0.037$ ). It should, however, be emphasized that for both phases of this study, a small amount of data was collected, which could mean that statistical testing was not completely reliable.

Next, we compared ${ }^{2} \mathrm{H}$-enrichment of sputum eosinophils with the blood eosinophils samples taken on the same day and from the same donor. This revealed that in placebo group (Figure 5C-D) and mepolizumab (Figure 5E-F), DNA from sputum eosinophils was overall lower enriched with deuterium compared to DNA from blood eosinophils. Since, we had a low amount of ${ }^{2} \mathrm{H}$-enrichment data from sputum eosinophils, we included sputum and blood data from an earlier eosinophilic asthma study (EHLA study, ABR NL44779.041.13). We combined this data with the data from both phases of the placebo group of this study to have as much as possible information about "normal" sputum eosinophil kinetics (Figure $5 \mathrm{G}$ ). This revealed that sputum eosinophils reached a ${ }^{2} \mathrm{H}$-plateau (with an overall lower ${ }^{2} \mathrm{H}$-enrichment) between day 6 and day 17 after label intake instead of a distinct peak as was observed in blood eosinophils at day 6-7 after label intake. Besides, the height of enrichment was overall lower in the sputum compared to blood $(\mathrm{P}=0.0002$ during the ublabelling and $\mathrm{P}=0.0004$ during the downlabelling ). 
A

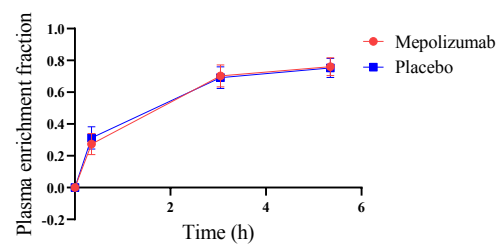

B

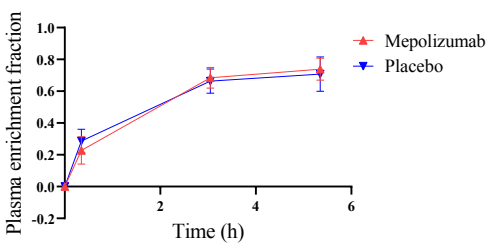

C

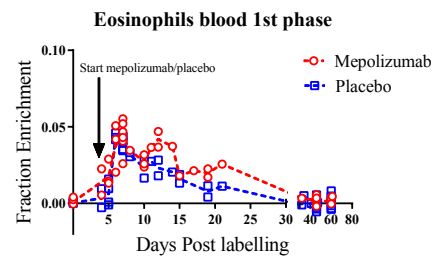

D

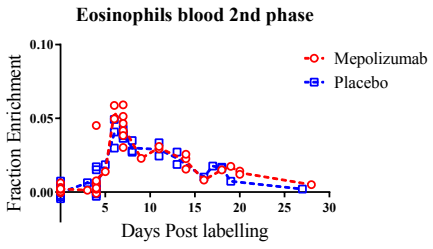

Uplabeling
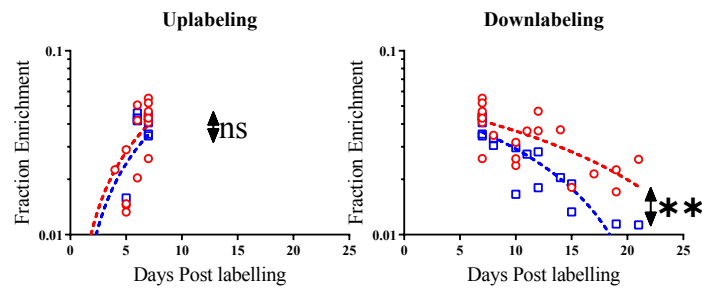

Uplabeling

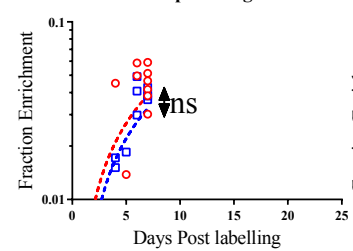

Downlabeling

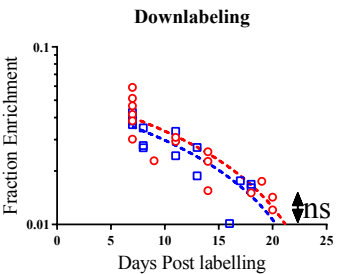

Figure 4. Deuterium enrichment of plasma and blood eosinophil DNA.

A. Deuterium enrichment of plasma glucose was measured before and at 3 time points during the intake of oral $6,6-{ }^{2} \mathrm{H}_{2}$-glucose to determine the availability of label for each patient in the mepolizumab (red) or placebo (blue) group. B. this was similarly done during the $2^{\text {nd }}$ phase of the study. $\mathrm{C}$. The ratio of ${ }^{2} \mathrm{H}-$ labelled and total DNA of blood eosinophils was determined before label intake and at several days after label intake for patients in the placebo (blue) and the mepolizumab group (red). This ratio was corrected for each individual's plasma glucose enrichment and the factor 0.65 (only $65 \%$ of the adenosine moieties can be enriched with deuterium when there is $100 \%$ deuterium availability). D. This was similarly done for blood eosinophils in both groups after the second label intake. In A-B median with interquartile range is plotted. In B-C the individual data points are shown for each time point and a dotted line connects the median values of each day with the other day. Arrow indicates the start of the first administration of mepolizumab or placebo at day 4 after first label intake. C-D A Simple linear regression was performed for the up labelling (day 0-7) and down labelling phases (day 8-22) separately to compare elevation and intercepts with the axes. NS not significant, $* * P \leq 0.01$. 
A Eosinophils sputum 1st phase

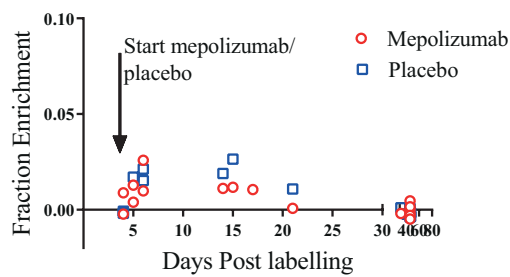

C

Eosinophils sputum and blood 1st phase

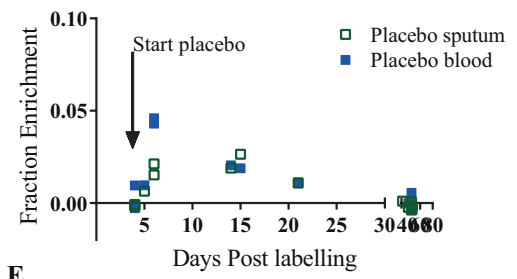

E

Eosinophils sputum and blood 1st phase

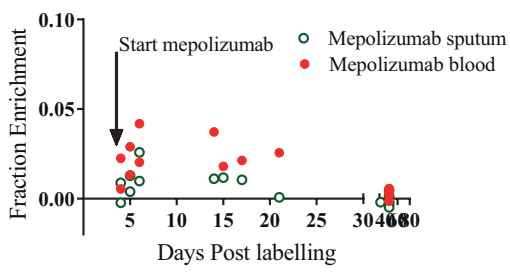

B

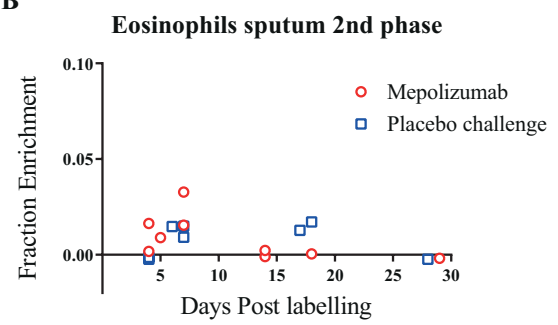

D

Eosinophils sputum and blood 2nd phase

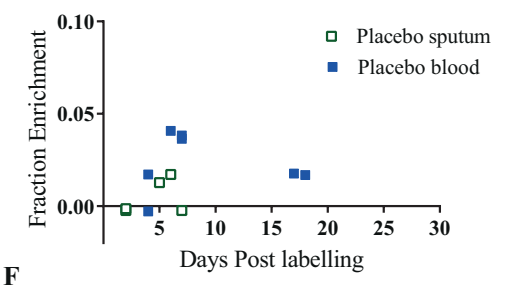

Eosinophils sputum and blood 2nd phase

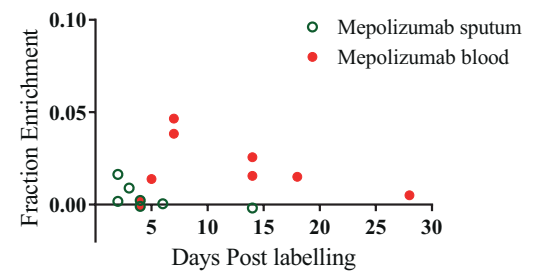

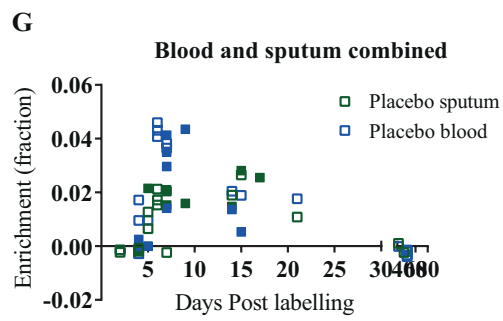

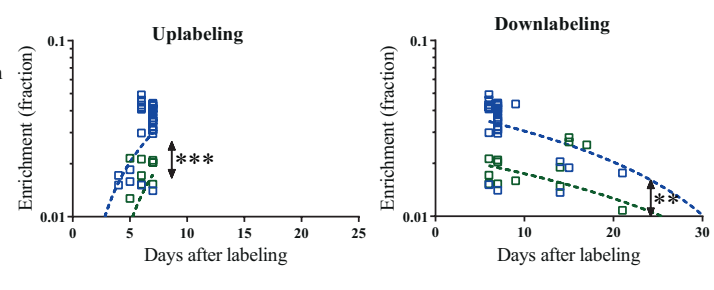

Figure 5. Deuterium enrichment of sputum eosinophil DNA and paired comparison to blood eosinophil DNA.

A. ${ }^{2} \mathrm{H}$-enrichment of DNA of sputum eosinophils was determined before label intake and at several days after label intake for patients in the placebo (blue) and the mepolizumab group (red) during the first labelling period. B. This was similarly done for sputum eosinophils during the second labelling period. The paired ${ }^{2} \mathrm{H}$-enrichment of blood eosinophil (blue) and sputum eosinophil (green) DNA is shown for the placebo group during the first labelling (C) and second labelling period (D). This was also done for the mepolizumab group (red is blood, green is sputum) during first labelling period (E) and the second labelling period (F). Individual data points are shown. Arrow indicates the start of the first administration of mepolizumab or placebo at day 4 after first label intake. G. The data points of $\mathbf{C}$ and $\mathbf{D}$ were combined 
(open blue squares represent blood eosinophils and open green squares represent the paired sputum samples) and plotted together with data points of a similar study $(n=9)$. These data points are indicated by closed squares. A Simple linear regression was performed for the up labelling (day 0-7) and down labelling (day 8-46) phases separately to compare elevation and intercepts with the axes. NS not significant, ${ }^{* *} \mathrm{P} \leq 0.01$.

\section{Basophil kinetics in blood}

Compared to blood eosinophils from the placebo group, appearance of ${ }^{2} \mathrm{H}$-label in basophils is later in time: day 4 versus day 6 after label intake, respectively (Figure $6 \mathrm{~A}$ and $6 \mathrm{~B}$ ). Also, a plateau of maximum enrichment was present from day 7-12 after label intake. After day 12 label is disappearing at a similar rate as was seen in blood eosinophils and from day 32 and beyond no ${ }^{2} \mathrm{H}$-label was measured any more (Figure $6 \mathrm{~A})$. Interestingly, the kinetics in the mepolizumab group was similar to the kinetics of basophils seen in the placebo group during both phases of the study.

$\mathbf{A}$

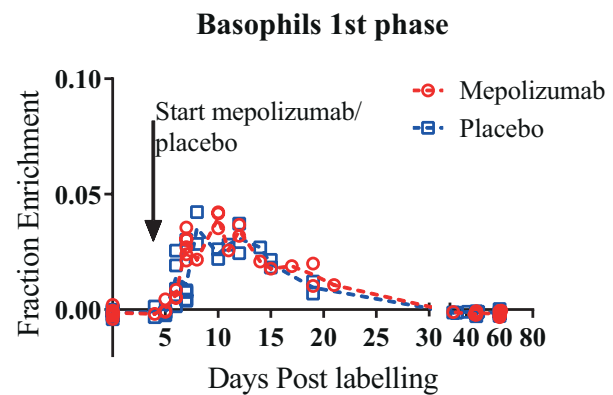

B

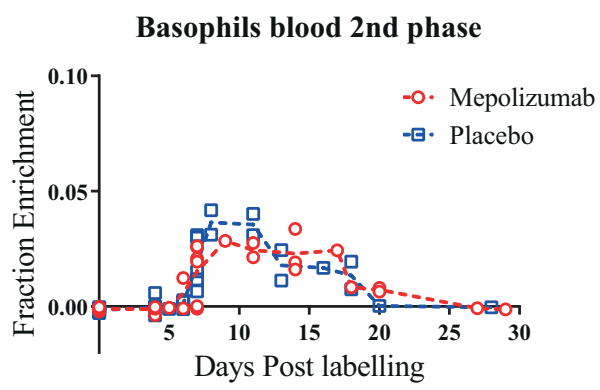

Figure 6. Deuterium enrichment blood basophil DNA.

A. ${ }^{2} \mathrm{H}$-enrichment of DNA of blood basophils was determined before label intake and at several days after label intake for patients in the placebo (blue) and the mepolizumab group (red) during the first labelling period. B. This was similarly done for DNA of blood basophils during the second labelling period. The individual data points are shown for each time point and a dotted line connects the median values of each day with the other day. Arrow indicates the start of the first administration of mepolizumab or placebo at day 4 after first label intake.

\section{Discussion}

To our knowledge, this is the first study in which a (non-toxic and an in vivo) labelling method was used to determine eosinophil and basophil kinetics in patients with asthma. The disadvantage of previous ex vivo labelling methods is that it causes granulocyte activation which in turn changes granulocyte homing characteristics ${ }^{23}$. As was explained in chapter 2 of this thesis, the kinetics of granulocytes is best described by a linear conveyor belt model in which a "first in, first out" principle is present. Unfortunately, so far, we have not developed a working mathematical model to fit the data with this principle. Therefore, this chapter will only interpret the change in kinetics rather than the calculation of lifespans. 


\section{Description of eosinophil kinetics in blood}

During the first phase DNA of eosinophils from both the placebo and the mepolizumab group contained label up to 22 days. Surprisingly, the loss of ${ }^{2} \mathrm{H}$-enrichment in the DNA of eosinophils in the mepolizumab group was slower from day 11 on despite a rapid decrease of the absolute number of eosinophils in the circulation (after 1-2 days after mepolizumab). This might be explained by at least three not mutually exclusive hypotheses.

\section{Delayed clearance and/or homing to the tissues}

The remaining eosinophils in the circulation are not as easily cleared from the circulation and/or homing to the (bronchial) tissues after blocking IL-5. We have delineated this in a simplified model in which labelled eosinophils enter the circulation in multiple waves, each one 4 days apart. Each wave is less labelled than the previous one, because of label dilution. When eosinophils exit the circulation with a delay, each of these waves is also delayed (compare supplementary Figure 5A with 5B).

Since, the absolute eosinophil count in the blood rapidly decreased after anti-IL-5 (already after 1 day, see Figure 2A), and taken into account that the post-mitotic pool transit time is at least 4 days (Figure 4A), the initial decrease of the circulatory eosinophils can only be accounted for by loss of eosinophils from the circulation, perhaps caused by rapid cell death ${ }^{29}$. This is supported by the fact that eosinophil maturation is not altered by anti-IL-5, at least not in mice ${ }^{30}$. The other remaining eosinophils might be less primed and not able to leave the circulation. In favor, of less priming is the upregulation of L-selectin on these cells (see Supplementary Figure 4A). Indeed, IL-5 is important to prime eosinophils for activation and transmigration to the airways ${ }^{31,32}$

\section{Delayed proliferation of eosinophil progenitors}

${ }^{2} \mathrm{H}$ enriched DNA from eosinophils in the bone marrow takes longer to completely be replaced/diluted by not ${ }^{2} \mathrm{H}$-labelled DNA, because the majority of eosinophil progenitors are proliferating slower in the bone marrow as a result of IL-5 blockade ${ }^{33}$. This mechanism, seems less likely at the first glance, because ${ }^{2} \mathrm{H}$-labelled DNA from eosinophils reached the circulation at a similar rate (after $4 / 5$ days) with a maximum peak at day 7-8. It should, however, be emphasized that treatment with mepolizumab started at day 4 , which means that anti-IL-5 effects on bone marrow eosinophils take longer to be evident in the peripheral blood. That is, slower eosinophil proliferation with label dilution happens in the bone marrow and since the post-mitotic pool transit time is at least 4 days (Figure $4 \mathrm{~A}$ ), there is a delay of at least 4 days before this change is seen in the circulation (compare supplementary Figure 5A with 5C).

\section{Other subset(s) of eosinophils}

A third possibility is the presence of a subset that remains present in the blood after short term treatment with mepolizumab. For these cells might have a slower turnover in the bone marrow which would account for the second wave of ${ }^{2} \mathrm{H}$-abelled DNA at around day 11/12 after label intake (see Figure 4C). The fact that IL-3R $\alpha$ expression decreased (Figure 3F), while CD62L expression increased (Supplementary Figure 4A) after treatment with mepolizumab, fits this hypothesis. As similar population of IL$3 \mathrm{R} \alpha^{\mathrm{dim}}$ and $\mathrm{CD} 62 \mathrm{~L}^{\mathrm{high}}$ eosinophils has been reported to be resident eosinophils, which 
seem less affected by anti-IL-5, at least in mice ${ }^{34}$. Following this logic, a third wave of eosinophils seems present after 20-22 days (Figure 4C). However, all putative subsets of eosinophils seem responsive to IL-5 as no differences were found between placebo and mepolizumab upon long term treatment with Mepolizumab. Interestingly, the same slow down-labelling and indications for three waves of eosinophils were also present after long term treatment (Figure 4D).

During the $2^{\text {nd }}$ phase (after 3 months of treatment with mepolizumab) the first labelled eosinophils again entered the circulation on day 4-5 after intake of label and the maximum amount of label in eosinophils was reached after 7-8 days. This pattern was similar between the mepolizumab and the placebo group. This indicates that the time for the first divided myelocytes to mature into eosinophils and to reach the circulation (post mitotic pool transit time, PMPtt) is around 4 days. So, there are no data that support the hypothesis that anti-IL-5 affects the maturation (time) for eosinophils. This is in line with previous work on murine eosinophils in which IL-5 withdrawal has very little if any effect on eosinophil maturation ${ }^{30}$. In contrast, it has been published that the absolute number of eosinophil myelocytes and metamyeloctes are attenuated after treatment with mepolizumab, while the number of early eosinophil committed progenitors (EoPs) are not ${ }^{15}$. These findings were explained by a maturation stop of eosinophilic myelocytes and metamyelocytes ${ }^{15}$. We, however, show that after 3 months of treatment with mepolizumab, both the PMPtt and the maximum height of ${ }^{2} \mathrm{H}$-enriched eosinophil DNA remains similar. This is not in line with an overall maturation stop or delay of all eosinophil (middle late) progenitors.

Taken together, it is more likely that IL-5 acts as a growth (proliferation) factor rather than a differentiation factor for mitotic IL-5R $\alpha$ eosinophil progenitors. The situation after prolonged treatment with Mepolizumab is the same as found in IL-5 KO mice ${ }^{12}$. The differentiation of eosinophils per se is not affected, only the absolute number of IL-5R $\alpha$ eosinophil progenitors (promyelocytes and myelocytes). This also explains the absence of reactive eosinophilia in both KO-mice and long term treatment with Mepolizumab ${ }^{12}$.

\section{Description of eosinophil kinetics in sputum}

Samples of sputum eosinophils in our study are more scarce compared to blood samples, but we are still able to make certain conclusions based on the available data. First, we found an overall lower abundance of ${ }^{2} \mathrm{H}$-label in the DNA of sputum eosinophil samples when compared to the blood eosinophil samples of the same donor (Figure $5 \mathrm{G})$. With other words: on day 7-28 after label intake eosinophil sputum samples showed a lower abundance of ${ }^{2} \mathrm{H}$-label. This finding can be explained by a possible mechanism. Eosinophils may remain longer in the airways (and therefore also in the sputum) compared to blood. This results in dilution of label, because unlabelled DNA from eosinophils remaining longer in the airways will 'dilute' the ${ }^{2} \mathrm{H}$ labelled DNA of eosinophils first appearing in the airways.

After treatment with mepolizumab, the situation in the airways is complex, because label disappearance in the sputum seems to be more rapid, especially after the $2^{\text {nd }}$ labelling period (after 80 days of treatment with mepolizumab). This cannot only be 
explained by slower homing of ${ }^{2} \mathrm{H}$-labelled eosinophils, because this would result in a longer existing of ${ }^{2} \mathrm{H}$-label in the sputum, which is not the case. On the other hand, if anti-IL-5 would only lead to a shorter survival of eosinophils in the airways than a higher ${ }^{2} \mathrm{H}$-label should be reached in the sputum at appearance on day 4-7 after label intake compared to blood or placebo from sputum samples, which is also not found. So most likely a combination of both mechanisms can account for the data found. So, antiIL-5 results in a slower homing of (labelled) eosinophils to the airways in combination with a fast turnover/ short survival of eosinophils in the lungs.

Our study has consequences for interpretation of old studies suggesting longevity of eosinophils in the lung tissue of patients with asthma ${ }^{16}$. The interpretation that eosinophils are long-lived ( $>2$ months) in the lung tissue of asthma patients is not supported by our data showing complete disappearance of label after $24-48$ days. The alternative explanations of persistent tissue eosinophilia in asthma patients treated with Mepolizumab might be: 1 . significant continuous homing of residual eosinophils to the lung tissue, 2. migration of eosinophil progenitors to the lung followed by local maturation and 3. preferential mobilization of resident eosinophils to the lung. However, whatever the mechanism there is no indication of long-lived eosinophils in the lung.

\section{Description of basophil kinetics in blood}

The number of basophils did not decline significantly (Figure 2B). This is in line with a recent published study ${ }^{35}$. We have now shown that also the basophil kinetics were not altered after mepolizumab. This is not completely surprising, because even though basophils are responsive to IL- $5^{36,37}$, their survival and proliferation is more potently regulated by $\mathrm{IL}-3^{38}$. In a different study in which $250-750 \mathrm{mg}$ of mepolizumab was administered basophil numbers were slightly attenuated after at least 8 weeks of treatment in asthmatics ${ }^{39,40}$. This difference might be caused by a higher sensitivity of the cells for IL-5. However, a more likely explanation is that automated haematology analysers are not sensitive enough to detect reliable levels of basophils in contrast to measurement by flow cytometry ${ }^{35}$.

Furthermore, we have reported that ${ }^{2} \mathrm{H}$-label coming from basophils is slower compared to blood eosinophils. This means that basophils have a longer post mitotic pool transit time (PMPtt). This is not in line with previous published work in which an equal to eosinophils PMPtt was found for basophils (around 4.3 days) ${ }^{17}$. The discrepancy between this study and the study of Steinbach et al. might be explained by the fact that the study of Steinbach et al. only enrolled patients with cancer ${ }^{17}$. It is possible that PMPtt of basophils is shortened by active systemic inflammation as has been found for neutrophils ${ }^{41}$. The fact that maximum enrichment in basophils reached a plateau on day 7-12, it is likely that kinetics in basophils is slower compared to eosinophils, as is reported in this study.

\section{In conclusion}

Treatment with Mepolizumab does not seem to affect differentiation of eosinophils, but rather suppresses the proliferation of eosinophil progenitors (promyelocytes and 
myelocytes). The remarkably slow down labelling of eosinophils in blood and sputum is consistent with multiple waves of eosinophils originating from progenitors with different kinetics. The striking similarities between treatment with mepolizumab, the presence of persistent eosinophilia in the lung tissue and the situation in IL-5-KO mice support the hypothesis that mepolizumab is particularly antagonizing reactive eosinophilia rather than block the maturation of the complete eosinophil compartment.

\section{Acknowledgements}

The authors thank Jeroen van Velzen and Pien van der Burght for help with the flow cytometry sorting. 


\section{References}

1. Bousquet J, Mantzouranis E, Cruz AA, Aït-Khaled N, Baena-Cagnani CE, Bleecker ER, et al. Uniform definition of asthma severity, control, and exacerbations: document presented for the World Health Organization Consultation on Severe Asthma. J Allergy Clin Immunol [Internet]. 2010 [cited 2019 Dec 5];126:926-38. Available from: http://www.ncbi.nIm.nih. gov/pubmed/20926125

2. To T, Stanojevic S, Moores G, Gershon AS, Bateman ED, Cruz AA, et al. Global asthma prevalence in adults: findings from the cross-sectional world health survey. BMC Public Health [Internet]. 2012 [cited 2019 Dec 5];12:204. Available from: http://www.ncbi.nlm. nih.gov/pubmed/22429515

3. Braman SS. The global burden of asthma. Chest [Internet]. 2006 [cited 2019 Dec 5];130:4S-12S. Available from: http://www.ncbi.nlm.nih.gov/pubmed/16840363

4. Haldar P, Pavord ID, Shaw DE, Berry MA, Thomas M, Brightling CE, et al. Cluster analysis and clinical asthma phenotypes. Am J Respir Crit Care Med [Internet]. 2008 [cited 2019 Dec 5];178:218-24. Available from: http://www.ncbi.nlm.nih.gov/pubmed/18480428

5. SJ, Vijverberg B, Hilvering JA, Raaijmakers JW, Lammers AH, Maitland-van der Zee L K. Clinical Utility of Asthma Biomarkers: From Bench to Bedside. Biologics [Internet]. 2013 [cited 2019 Dec 5];7. Available from: https://pubmed.ncbi.nlm.nih.gov/24009412-clinical-utility-of-asthmabiomarkers-from-bench-to-bedside/?from_term=hilvering+and+vijverberg\&from_pos=2

6. Simpson JL, Scott R, Boyle MJ, Gibson PG. Inflammatory subtypes in asthma: assessment and identification using induced sputum. Respirology [Internet]. 2006 [cited 2019 Dec 5];11:54-61. Available from: http://www.ncbi.nlm.nih.gov/pubmed/16423202

7. Haldar P, Brightling CE, Hargadon B, Gupta S, Monteiro W, Sousa A, et al. Mepolizumab and exacerbations of refractory eosinophilic asthma. N Engl J Med [Internet]. 2009;360:973-84. Available from: http://www.ncbi.nlm.nih.gov/pubmed/19264686

8. Hassani M, Koenderman L. Immunological and hematological effects of IL-5(R $\alpha)$-targeted therapy: An overview. Allergy [Internet]. 2018 [cited 2019 Nov 7];73:1979-88. Available from: http://www.ncbi.nlm.nih.gov/pubmed/29611207

9. Kolbeck R, Kozhich A, Koike M, Peng L, Andersson CK, Damschroder MM, et al. MEDI-563, a humanized anti-IL-5 receptor alpha mAb with enhanced antibody-dependent cell-mediated cytotoxicity function. J Allergy Clin Immunol [Internet]. 2010;125:1344-53. Available from: http://www.ncbi.nlm.nih.gov/pubmed/20513525

10. Takatsu K. Interleukin-5 and IL-5 receptor in health and diseases. Proc Jpn Acad Ser B Phys Biol Sci [Internet]. 2011;87:463-85. Available from: http://www.ncbi.nlm.nih.gov/ pubmed/21986312

11. Smith SG, Chen R, Kjarsgaard M, Huang C, Oliveria J-P, O'Byrne PM, et al. Increased numbers of activated group 2 innate lymphoid cells in the airways of patients with severe asthma and persistent airway eosinophilia. J Allergy Clin Immunol [Internet]. 2016 [cited $2020 \mathrm{Mar}$ 12];137:75-86.e8. Available from: http://www.ncbi.nlm.nih.gov/pubmed/26194544

12. Foster PS, Hogan SP, Ramsay AJ, Matthaei KI, Young IG. Interleukin 5 deficiency abolishes eosinophilia, airways hyperreactivity, and lung damage in a mouse asthma model. J Exp Med [Internet]. 1996 [cited 2020 Mar 12];183:195-201. Available from: http://www.ncbi. nlm.nih.gov/pubmed/8551223 
13. Sanderson CJ. Interleukin-5, eosinophils, and disease. Blood [Internet]. 1992;79:3101-9. Available from: http://www.ncbi.nlm.nih.gov/pubmed/1596561

14. Broughton SE, Nero TL, Dhagat U, Kan WL, Hercus TR, Tvorogov D, et al. The betac receptor family - Structural insights and their functional implications. Cytokine [Internet]. 2015;74:247-58. Available from: http://www.ncbi.nlm.nih.gov/pubmed/25982846

15. Menzies-Gow A, Flood-Page P, Sehmi R, Burman J, Hamid Q, Robinson DS, et al. AntiIL-5 (mepolizumab) therapy induces bone marrow eosinophil maturational arrest and decreases eosinophil progenitors in the bronchial mucosa of atopic asthmatics. J Allergy Clin Immunol [Internet]. 2003;111:714-9. Available from: http://www.ncbi.nIm.nih.gov/ pubmed/12704348

16. Flood-Page PT, Menzies-Gow AN, Kay AB, Robinson DS. Eosinophil's role remains uncertain as anti-interleukin-5 only partially depletes numbers in asthmatic airway. Am J Respir Crit Care Med [Internet]. 2003;167:199-204. Available from: http://www.ncbi.nIm.nih.gov/ pubmed/12406833

17. Steinbach KH, Schick P, Trepel F, Raffler H, Dohrmann J, Heilgeist G, et al. Estimation of kinetic parameters of neutrophilic, eosinophilic, and basophilic granulocytes in human blood. Blut [Internet]. 1979;39:27-38. Available from: http://www.ncbi.nlm.nih.gov/pubmed/223692

18. Cartwright GE, Athens JW, Wintrobe MM. The kinetics of granulopoiesis in normal man. Blood [Internet]. 1964 [cited 2018 Jul 26];24:780-803. Available from: http://www.ncbi. nlm.nih.gov/pubmed/14235362

19. Geering B, Stoeckle C, Conus S, Simon H-U. Living and dying for inflammation: neutrophils, eosinophils, basophils. Trends Immunol [Internet]. 2013 [cited 2018 Feb 28];34:398-409. Available from: http://linkinghub.elsevier.com/retrieve/pii/S1471490613000562

20. Lukawska JJ, Livieratos L, Sawyer BM, Lee T, O'Doherty M, Blower PJ, et al. Real-time differential tracking of human neutrophil and eosinophil migration in vivo. J Allergy Clin Immunol [Internet]. 2014 [cited 2018 Feb 27];133:233-239.e1. Available from: http://www. ncbi.nlm.nih.gov/pubmed/23953710

21. Parwaresch MR, Walle AJ, Arndt D. The peripheral kinetics of human radiolabelled eosinophils. Virchows Arch B Cell Pathol [Internet]. 1976;57-66. Available from: http:// www.ncbi.nlm.nih.gov/pubmed/821244

22. Dale DC, Hubert RT, Fauci A. Eosinophil kinetics in the hypereosinophilic syndrome. J Lab Clin Med [Internet]. 1976;87:487-95. Available from: http://www.ncbi.nlm.nih.gov/ pubmed/1249478

23. Farahi N, Singh NR, Heard S, Loutsios C, Summers C, Solanki CK, et al. Use of 111-Indiumlabeled autologous eosinophils to establish the in vivo kinetics of human eosinophils in healthy subjects. Blood [Internet]. 2012;120:4068-71. Available from: http://www.ncbi. nlm.nih.gov/pubmed/22993388

24. Macallan DC, Fullerton CA, Neese RA, Haddock K, Park SS, Hellerstein MK. Measurement of cell proliferation by labelling of DNA with stable isotope-labeled glucose: studies in vitro, in animals, and in humans. Proc Natl Acad Sci U S A [Internet]. 1998 [cited $2018 \mathrm{Jul}$ 30];95:708-13. Available from: http://www.ncbi.nlm.nih.gov/pubmed/9435257

25. Macallan DC, Asquith B, Zhang Y, de Lara C, Ghattas H, Defoiche J, et al. Measurement of proliferation and disappearance of rapid turnover cell populations in human studies using deuterium-labeled glucose. Nat Protoc [Internet]. 2009 [cited 2019 Sep 3];4:1313-27. Available from: http://www.nature.com/articles/nprot.2009.117 
26. Rutgers SR, Timens W, Kaufmann HF, van der Mark TW, Koëter GH, Postma DS. Comparison of induced sputum with bronchial wash, bronchoalveolar lavage and bronchial biopsies in COPD. Eur Respir J [Internet]. 2000 [cited 2019 Sep 3];15:109-15. Available from: http://www. ncbi.nlm.nih.gov/pubmed/10678630

27. Hassani M, vanStaverenS, van Grinsven E, Bartels M, TesselaarK, Leijte G, etal. Characterization of the phenotype of human eosinophils and their progenitors in the bone marrow of healthy individuals. Haematologica [Internet]. 2019 [cited 2019 Sep 19]; haematol.2019.219048. Available from: http://www.ncbi.nlm.nih.gov/pubmed/31101758

28. Busch R, Neese RA, Awada M, Hayes GM, Hellerstein MK. Measurement of cell proliferation by heavy water labelling. Nat Protoc [Internet]. 2007 [cited 2019 Sep 3];2:3045-57. Available from: http://www.ncbi.nlm.nih.gov/pubmed/18079703

29. Xu J, Jiang F, Nayeri F, Zetterstrom O. Apoptotic eosinophils in sputum from asthmatic patients correlate negatively with levels of IL-5 and eotaxin. Respir Med [Internet]. 2007;101:144754. Available from: http://www.ncbi.nlm.nih.gov/pubmed/17379492

30. Fulkerson PC, Schollaert KL, Bouffi C, Rothenberg ME. IL-5 triggers a cooperative cytokine network that promotes eosinophil precursor maturation. J Immunol [Internet]. 2014 [cited 2020 Jan 9];193:4043-52. Available from: http://www.ncbi.nlm.nih.gov/pubmed/25230753

31. Johansson MW, Gunderson KA, Kelly EA, Denlinger LC, Jarjour NN, Mosher DF. Anti-IL-5 attenuates activation and surface density of beta(2) -integrins on circulating eosinophils after segmental antigen challenge. Clin Exp Allergy [Internet]. 2013;43:292-303. Available from: http://www.ncbi.nlm.nih.gov/pubmed/23414537

32. Stein ML, Villanueva JM, Buckmeier BK, Yamada Y, Filipovich AH, Assa'ad AH, et al. AntiIL-5 (mepolizumab) therapy reduces eosinophil activation ex vivo and increases IL-5 and IL-5 receptor levels. J Allergy Clin Immunol [Internet]. 2008;121:1473-1483,1483. Available from: http://www.ncbi.nlm.nih.gov/pubmed/18410960

33. Sanderson CJ, Warren DJ, Strath M. Identification of a lymphokine that stimulates eosinophil differentiation in vitro. Its relationship to interleukin 3, and functional properties of eosinophils produced in cultures. J Exp Med [Internet]. 1985;162:60-74. Available from: http://www.ncbi.nlm.nih.gov/pubmed/3925072

34. Mesnil C, Raulier S, Paulissen G, Xiao X, Birrell MA, Pirottin D, et al. Lung-resident eosinophils represent a distinct regulatory eosinophil subset. J Clin Invest [Internet]. 2016;126:327995. Available from: http://www.ncbi.nlm.nih.gov/pubmed/27548519

35. Wright AKA, Diver S, McCarthy J, Marvin A, Soares M, Thornton T, et al. Mepolizumab does not alter the blood basophil count in severe asthma. Allergy [Internet]. 2019 [cited 2019 Dec 11];all.13879. Available from: https://onlinelibrary.wiley.com/doi/abs/10.1111/all.13879

36. Denburg JA, Silver JE, Abrams JS. Interleukin-5 is a human basophilopoietin: induction of histamine content and basophilic differentiation of $\mathrm{HL}-60$ cells and of peripheral blood basophil-eosinophil progenitors. Blood [Internet]. 1991;77:1462-8. Available from: http:// www.ncbi.nlm.nih.gov/pubmed/1706953

37. Bischoff SC, Brunner T, De Weck AL, Dahinden CA. Interleukin 5 modifies histamine release and leukotriene generation by human basophils in response to diverse agonists. J Exp Med [Internet]. 1990 [cited 2019 Dec 11];172:1577-82. Available from: http://www.ncbi.nlm. nih.gov/pubmed/1701820

38. Tanimoto $\mathrm{Y}$, Takahashi K, Kimura I. Effects of cytokines on human basophil chemotaxis. Clin Exp Allergy [Internet]. 1992;22:1020-5. Available from: http://www.ncbi.nIm.nih.gov/ pubmed/1334781 
39. Buttner C, Lun A, Splettstoesser T, Kunkel G, Renz H. Monoclonal anti-interleukin- 5 treatment suppresses eosinophil but not T-cell functions. Eur Respir J [Internet]. 2003;21:799-803. Available from: http://www.ncbi.nlm.nih.gov/pubmed/12765424

40. Nair P, Pizzichini MMM, Kjarsgaard M, Inman MD, Efthimiadis A, Pizzichini $E$, et al. Mepolizumab for prednisone-dependent asthma with sputum eosinophilia. N Engl J Med [Internet]. 2009 [cited 2020 Jan 13];360:985-93. Available from: http://www.ncbi.nlm.nih. gov/pubmed/19264687

41. Terashima T, Wiggs B, English D, Hogg JC, van Eeden SF. Polymorphonuclear leukocyte transit times in bone marrow during streptococcal pneumonia. Am J Physiol [Internet]. 1996 [cited 2020 Jan 14];271:L587-92. Available from: http://www.ncbi.nlm.nih.gov/pubmed/8897906

42. Mori Y, Iwasaki H, Kohno K, Yoshimoto G, Kikushige Y, Okeda A, et al. Identification of the human eosinophil lineage-committed progenitor: revision of phenotypic definition of the human common myeloid progenitor. J Exp Med [Internet]. 2009;206:183-93. Available from: http://www.ncbi.nlm.nih.gov/pubmed/19114669

43. Sehmi R, Smith SG, Kjarsgaard M, Radford K, Boulet LP, Lemiere C, et al. Role of local eosinophilopoietic processes in the development of airway eosinophilia in prednisonedependent severe asthma. Clin Exp Allergy [Internet]. 2016;46:793-802. Available from: http://www.ncbi.nlm.nih.gov/pubmed/26685004 
A

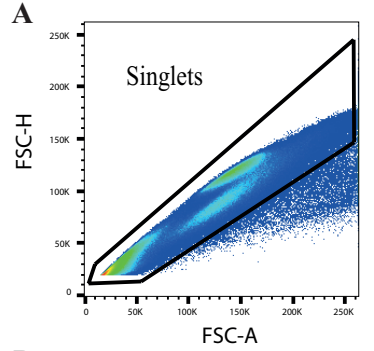

D

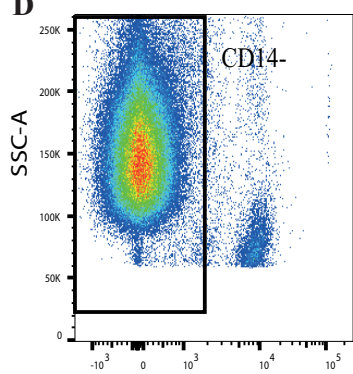

CD14 APC-H7
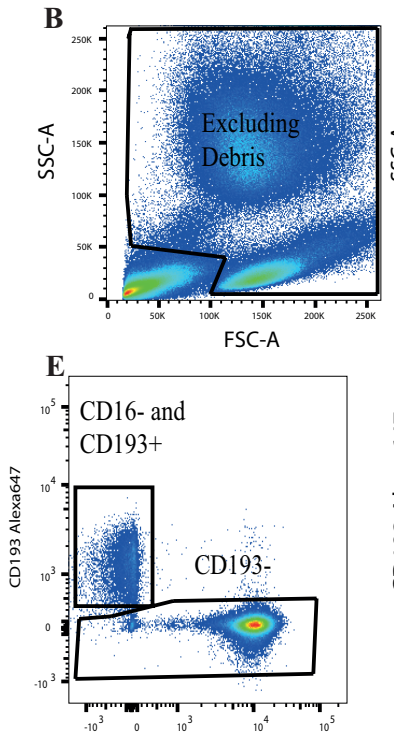

CD16 PE-Cy7

G

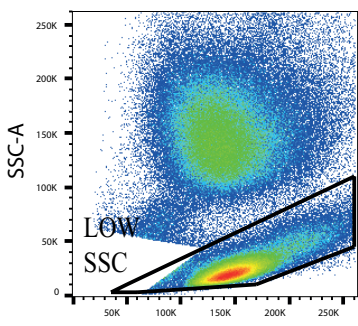

FSC-A

$\mathbf{H}$

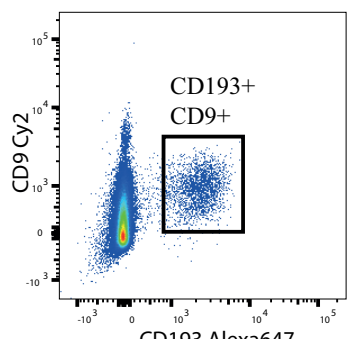

C

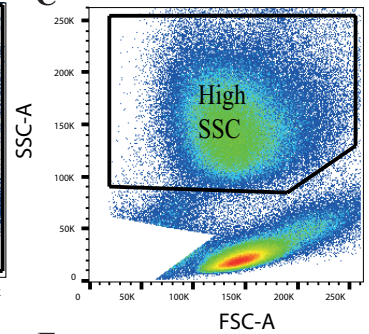

F
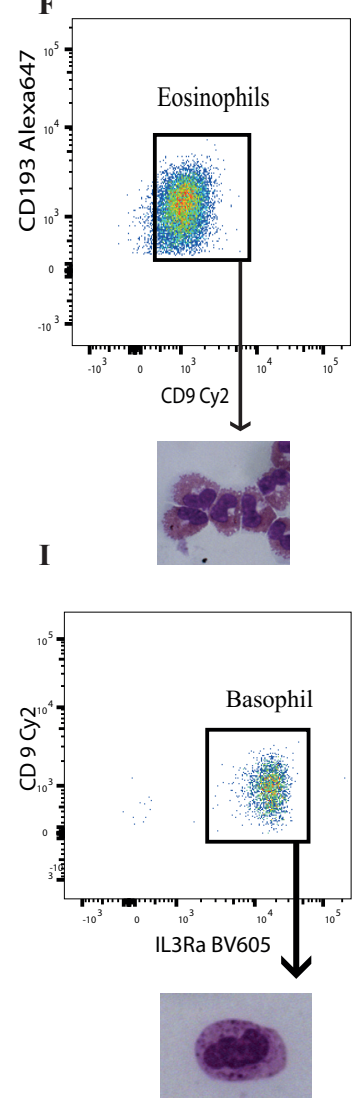

\section{Supplementary Figure 1. Gating strategy for FACS sorting eosinophils and basophils in the blood.}

Blood eosinophils were identified by first gating on singlets on the basis of FSC-A and FSC-H (A), debris is excluded on the basis of FSC-A and SSC-A (B), subsequently only high SSC-A cells are gated (C), CD14 high cells are excluded (D), CD16 ${ }^{\text {neg }}$ and $C D 193^{\text {pos }}$ cells are gated $(E)$ and finally within the later gate $C D 9^{\text {pos }}$ cells are gated (F). These cells were FACS sorted and cytospins were stained with May-Grünwald Giesma (MMG) and microscopically evaluated. An example of a representative microscopic image (objective 100x) of a cytospin of sorted eosinophils is shown. Basophils were sorted from the same samples, but this time SSClow cells are gated $(\mathbf{G})$, followed by $\mathrm{CD} 9^{\text {pos }}$ and $\mathrm{CD} 193^{\text {pos }}(\mathbf{H})$ and finally gated for IL-3R $\alpha^{\text {high }}$ to obtain basophils (H). Also, a representative (MMG stained) microscopic image (objective 100x) of sorted basophils is shown. 
A

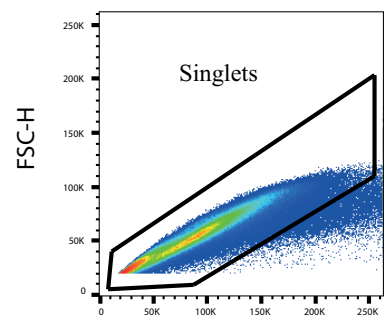

FSC-A

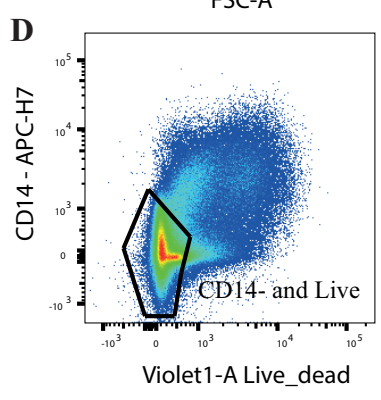

B
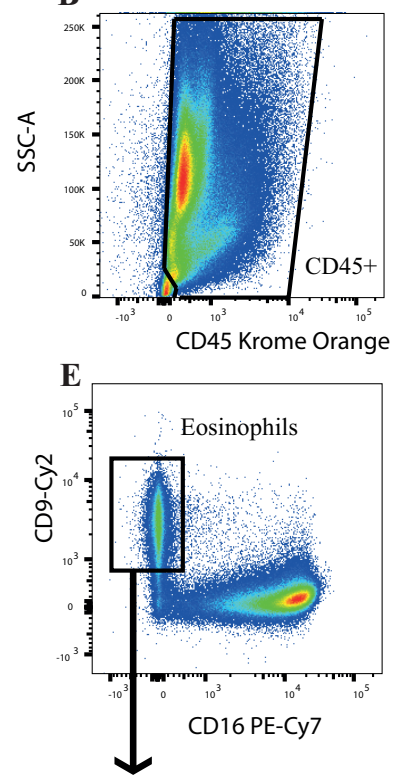

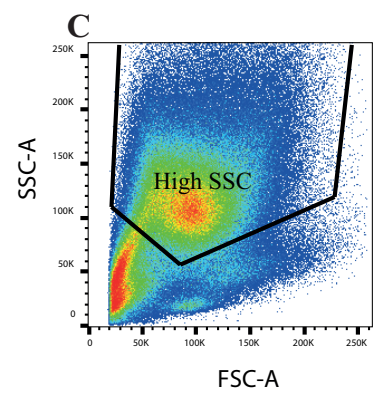

FSC-A

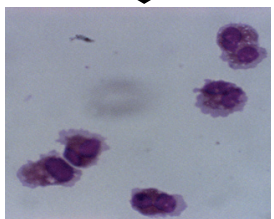

\section{Supplementary Figure 2. Gating strategy for FACS sorting eosinophils in the sputum.}

Sputum eosinophil were gated after only gating singlets (A), CD45 pos cells (B), SSC high cells (C) and CD14 ${ }^{\text {neg }}$ and negative for live/dead stain (D). Finally, eosinophils were gated on the basis of CD16 $6^{\text {neg }}$ and $C D 9^{\text {pos }}$ cells and FACS sorted (E). A representative microscopic image (100x objective) of an MMG stained cytospin of sorted eosinophils is shown. 
A

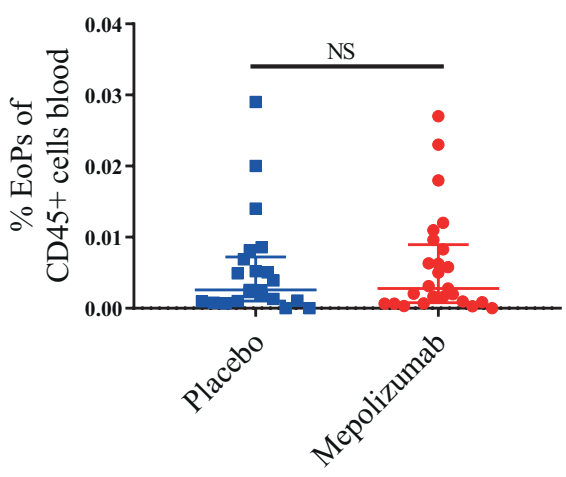

B

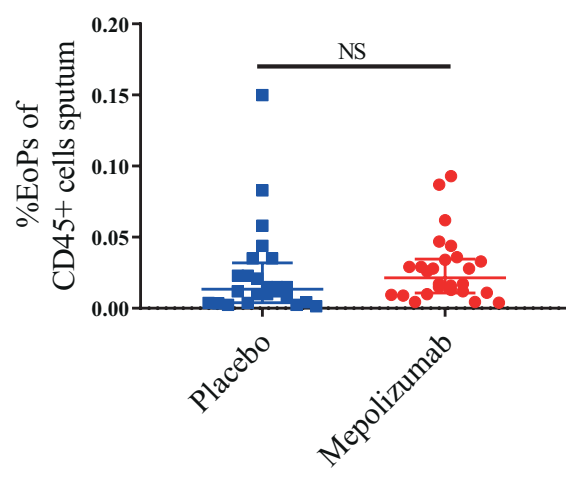

Supplementary Figure 3. Number of EoPs in blood and sputum.

EoPs in the blood (A) and sputum (B) were recognized, gated and enumerated as a percentage of total white blood cells as was published befor $\mathrm{e}^{42,43}$. Single data points with median and interquartile range are shown for mepolizumab (red) and placebo (blue). A Mann-Whitney test was performed to compare both groups. NS not significant. 
A

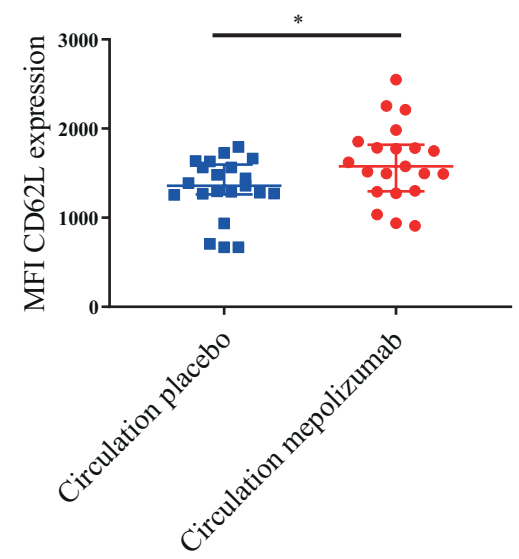

B

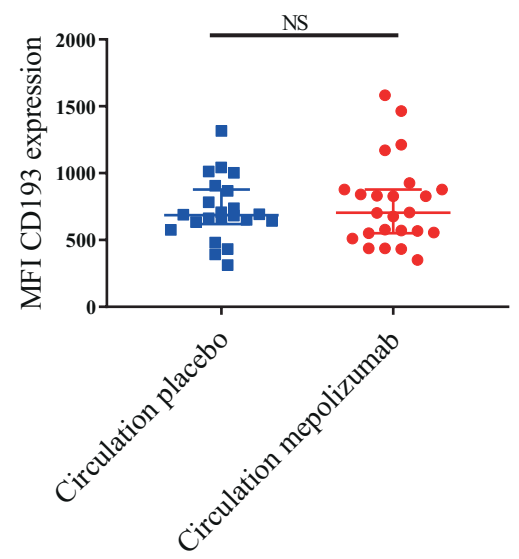

Supplementary Figure 4. Median fluorescence intensity for CD62L and CD193 in blood eosinophils. The MFI for CD62L membrane expression (A) and CD193 membrane expression (B) is plotted for multiple samples after at least 3 days of treatment with placebo (blue) and mepolizumab (red) after. A MannWhitney test was used to compare both treatment groups. Individual data points with median and interquartile range is shown. NS not significant and $* P \leq 0.05$. 
A

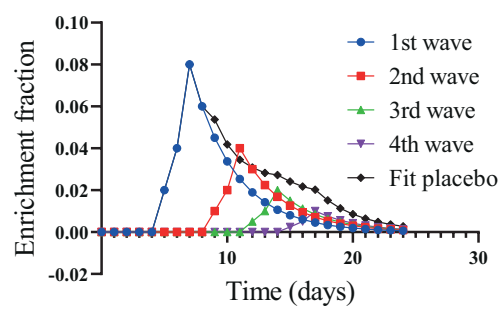

C

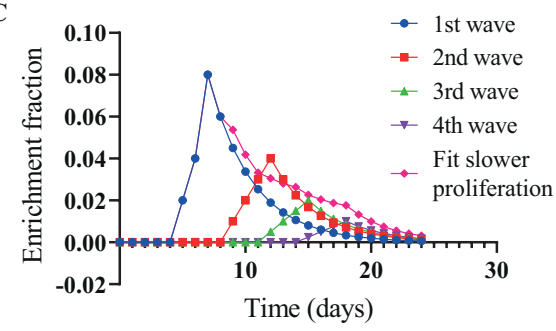

B
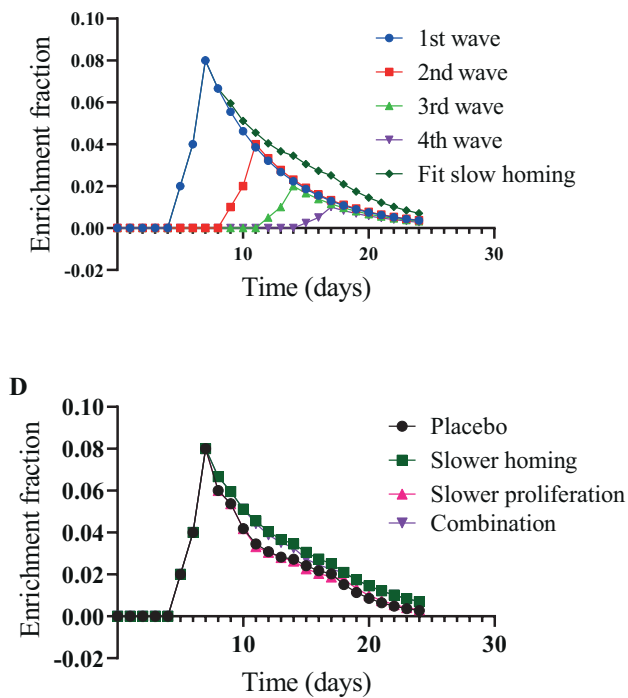

Supplementary Figure 5. Schematic model for blood eosinophil kinetics.

A. A schematic model of eosinophil kinetics in the blood is shown. This model assumes that 4 waves of ${ }^{2} \mathrm{H}$-labelled DNA of eosinophils from the bone marrow enter the circulation consecutively with a fixed time between each wave ( 4 days). Every wave reaches half of the maximum deuterium label of the previous wave because of half of loss of label with each division. Because the absolute cell number in the placebo group remains the same, the number of cells that enter the circulation are in equilibrium with the number of cells leaving the circulation. Blue is $1^{\text {st }}$ wave, red is $2^{\text {nd }}$ wave, green is $3^{\text {rd }}$ wave and purple is $4^{\text {th }}$ wave. Black dots represent the enrichment when enrichment is corrected for all 4 waves. B. similar as in A, but this time cells are lost from the circulation slower than in A (lifespan of 6 days instead of 4 days. C. similar as in A but because of slower proliferation in the bone marrow, the maximum amount of deuterium enrichment takes 1 day longer for each wave, except for wave 1 . D. the fits for all separate enrichment curves have been put in one graph. The purple points represent the combination of fits of C and D. 


\title{
Chapter 10
}

\section{The effect of dupilumab on eosinophil homing and activation in patients with atopic dermatitis}

\author{
Marwan Hassani ${ }^{1,2}$, Daphne S. Bakker, ${ }^{2,3}$, Edward F. Knol ${ }^{2,3}$, \\ Marjolein S. de Bruin-Weller ${ }^{3}$, Leo Koenderman ${ }^{1,2}$, Judith L. Thijs ${ }^{3}$
}

1. Department of Respiratory Medicine, University Medical Center Utrecht, The Netherlands.

2. Center for Translational Immunology, University Medical Center Utrecht, The Netherlands

3. Department of Dermatology and Allergology, University Medical Center Utrecht, The Netherlands. 


\section{Abstract}

Atopic dermatitis (AD) is classically characterized by type 2 inflammation, and treatment with dupilumab, an antibody against IL-4R $\alpha$, improves clinical signs and symptoms of AD patients. The role of one of the type2 effector cells, eosinophils, in this disease is largely unknown. Also, other than a (transient) eosinophilia in the peripheral blood, little is known about how treatment with dupilumab affects the functionality of these cells in AD.

We characterized the activation state of eosinophils and neutrophils in patients with $A D$ before and after treatment with dupilumab, by measuring multiple surface markers using flow cytometry. We also compared the activation state of eosinophils of $A D$ patients with cells from healthy donors.

Eosinophils from blood of AD patients showed an increased activation state of blood when compared to cells of healthy donors. This activation state did not change after treatment with dupilumab. On the other side, a transient increase of expression of CCR3 (CD193) expression was present with a peak after 4 weeks of treatment. In addition, a sustained increase of CD44 expression was detected 4 and 16 weeks after treatment with dupilumab. In marked contrast, neutrophils in peripheral blood did not show any clear signs of activation.

Our findings might imply that dupilumab caused a decrease in homing of these cells to the tissue. Since these cells exhibited an activated phenotype, this situation might proof hazardous because of collateral damage by aspecific activation of these very cytotoxic cells. Therefore, it might be sensible to monitor eosinophil counts and activation state during treatment with dupilumab. 


\section{Introduction}

Eosinophils are innate immune cells that play a role in the defense against invading multicellular parasites, particularly helminths ${ }^{1}$. When these cells are activated, they release reactive oxygen species and eosinophilic granule proteins that synergize in the killing of these parasites ${ }^{2}$. These toxic mediators are also released in pathological (atopic) conditions where they can cause collateral damage to healthy tissues ${ }^{3}$.

Atopic dermatitis (AD) is a pruritic and eczematous skin disorder that is classically characterized by type 2 inflammation and is associated with increased production of IL-4, IL-5, and IL-134. However, the role of the eosinophils, known as T2-effector cells, in $A D$ has been a subject of debate for years ${ }^{5}$. Even though enough evidence has been provided that eosinophil toxic granular proteins are released in the skin of AD patients ${ }^{5,6}$, short term treatment with anti-IL-5 (mepolizumab) did not result in improvement of symptoms of patients with $A D$, despite a decrease of circulatory eosinophils ${ }^{7}$.

In contrast, treatment of patients with a human monoclonal antibody against the interleukin-4 receptor alpha chain, dupilumab, did result in improvement of both symptoms and objective clinical signs of $A D^{8}$. This improvement was seen, despite a transient increase of eosinophil numbers during dupilumab treatment in the circulation ${ }^{8,9}$. This increase in eosinophil numbers in the circulation is accompanied with a decrease of eotaxin-1 and eotaxin-3 in serum without an increase of IL-5 levels ${ }^{10}$. A similar finding was obtained in a study where dupilumab was tested in a cohort of patients with chronic rhinosinusitis with nasal polyposis ${ }^{11}$. In this study, tissue levels of eotaxin-1, -2 and -3 were reduced locally in the nasal tissue. This decrease was associated with lower levels of eosinophilic cationic protein (ECP) in the nasal tissue as well. These findings strongly suggested that eosinophil numbers in the blood increased because of reduced homing of eosinophils to the tissues, rather than an increase in eosinophilopoiesis. It is unknown if eosinophils in dupilumab treated $A D$ patients are activated, which might lead to tissue damage. Therefore, we tested if circulatory eosinophils in AD patients treated with dupilumab are characterized by signs of increased priming or degranulation.

\section{Materials and Methods}

\section{Subjects and study design}

This study was part of a larger prospective, observational cohort study in which patients with moderate to severe AD were enrolled in the Dutch BioDay Registry at the National Expertise Center for Atopic Dermatitis from the University Medical Center Utrecht (UMCU). For this study, 16 patients with moderate to severe AD were enrolled between September 2018 and April 2019. Patients were treated with a subcutaneous dose of $600 \mathrm{mg}$ dupilumab at start of treatment, followed by $300 \mathrm{mg}$ every other week (mostly self-administered). A schematic overview of the study procedures is provided in Figure 1. For most patients, systemic immunosuppressive treatment was discontinued before starting dupilumab treatment, but concomitant treatment with topical corticosteroids was allowed. Blood samples were collected at three different time points: at baseline prior to treatment with dupilumab and after 4 weeks and 16 weeks of treatment with dupilumab. This study did not fall under the scope of the Medical Research Involving 
Human Subjects Act which was confirmed by the local Medical Research Ethics Committee (METC 18/239). Informed consent was collected from each patient. The study was performed according to the declaration of Helsinki.

\section{Sample processing and stimulation}

Blood was collected in sodium heparin tubes (Vacuette ${ }^{\circledR}$ Greiner bio-one, Kremsmünster, Austria). Subsequently whole blood was either stimulated with $1 \mu \mathrm{M}$-formylmethionineleucyl-phenylalaline (fMLF) (Sigma-Aldrich, St. Louis, USA) in a $37^{\circ} \mathrm{C}$ water bath for 10 minutes or left on the bench for 10 minutes. Thereafter, erythrocytes were lysed using an ice-cold lysis buffer ( $150 \mathrm{mM} \mathrm{NH}_{4} \mathrm{Cl}, 10 \mathrm{mM} \mathrm{KHCO}_{3}$ and $0.1 \mathrm{mM} \mathrm{NA}_{2}$ EDTA). After this, the remaining leukocytes were washed and resuspended in a staining buffer consisting of PBS supplemented with $0.32 \% \mathrm{w} / \mathrm{v}$ trisodium citrate (prepared by the pharmacy of the University Medical Centre Utrecht) and 10\% w/v human plasma albumin (Sanquin, Amsterdam, the Netherlands).

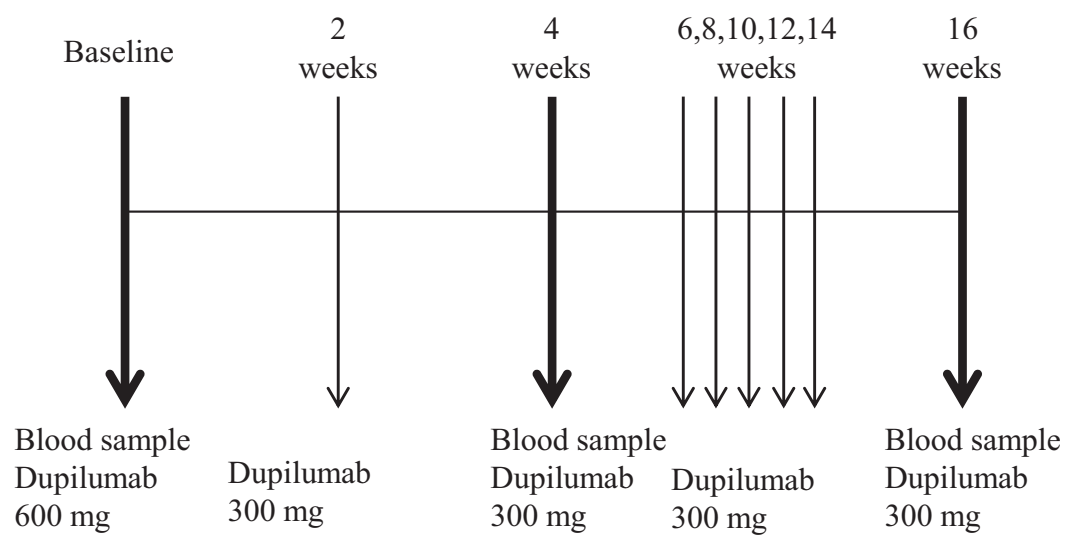

Figure 1. Study design.

16 patients with severe atopic dermatitis were enrolled at the university medical center in Utrecht. Patients were treated with subcutaneous dose of dupilumab at the start of the study (600 mg), followed by subcutaneous doses each 2 weeks ( $300 \mathrm{mg}$ ). A blood sample was taken at baseline, after 4 weeks and after 16 weeks of treatment (indicated by bold arrows).

\section{Flow cytometry}

One million cells at a concentration of 40 million cells per $\mathrm{mL}$ were stained with antibodies for 30 minutes on ice, and washed twice before analysis on the LSR-Fortessa flow cytometer (BD, Mountain View CA, USA). The following antibodies were used for staining: from BD: CD44-FITC (clone L178), CD66b-PerCP-Cy 5.5 (clone G10F5), CD35AF647 (clone E11) and CD11b-AF700 (clone ICRF44); from Biolegend: CD193-BV605 (clone 5E8) and CD62L-APC-Cy7 (clone DREG-56); from Sony Biotechnology: CD16BV785 (clone 3G8) and CD14-PB (clone HCD14).

Granulocytes in blood were identified on the basis of FSC/SSC (Supplementary Figure 1C). After that monocytes were excluded using CD14 (Supplementary Figure 1D). Finally, 
eosinophils were identified as CD16 $6^{\text {neg }}$ and CD193 ${ }^{\text {high }}$ cells and neutrophils identified as CD16 ${ }^{\text {high }}$ and CD193 ${ }^{\text {neg }}$ cells (Supplementary Figure 1E).

\section{Data analysis and representation}

FlowJo v10 (LLC, Ashland, OR, USA) was used to analyze the flow cytometry data. Statistical analysis was performed using GraphPad Prism 7.04 (GraphPad Software, La Jolla, CA, USA). Data are presented as median \pm interquartile range. A Wilcoxon matched-pairs signed rank test was performed to compare two paired groups and a Mann-Whitney test was used for non-paired analysis. For comparisons between more than two groups, a Friedman tests or a Kruskal-Wallis test was used. A P value $\leq 0.05$ was considered statistically significant. Graphs and figures were modified using Adobe Illustrator CS6 v16.0.0 (Adobe Systems, San Jose, CA, USA).

\section{Results and Discussion}

\section{Patients characteristics}

Sixteen AD patients were enrolled for this study (median EASI 14.9, IQR 10.6-18.5), (Table 1). Although the sample size to address clinical differences was (too) small, most patients showed an improvement in both EASI and IGA score after treatment. No significant rise in eosinophil numbers in the circulation was detected, albeit eosinophilia ( $\geq 0.45 \times 10^{6}$ cells $/ \mathrm{ml}$ ) was detected after 4 - 16 weeks of dupilumab treatment in some patients. During the course of the study there were no adverse events. The lack of a significant eosinophilia in this small group might be caused by chance. Alternatively, eosinophilia might be linked to an endotype that has not been elucidated yet.

\begin{tabular}{|c|c|c|c|c|}
\hline Patient characteristics & At baseline & $\begin{array}{l}\text { After } 4 \\
\text { weeks }\end{array}$ & $\begin{array}{l}\text { After } 16 \\
\text { weeks }\end{array}$ & $\begin{array}{l}\text { P-value } \\
\text { (baseline- } \\
16 \text { weeks) }\end{array}$ \\
\hline N & 16 & 16 & 14 & N/A \\
\hline Female, N (\%) & $5(31.3 \%)$ & $5(31.3 \%)$ & $3(21.4 \%)$ & N/A \\
\hline $\begin{array}{l}\text { Age (years) } \\
\text { Median [IQR] }\end{array}$ & $\begin{array}{l}46 \\
{[28-56]}\end{array}$ & $\begin{array}{l}46 \\
{[28-56]}\end{array}$ & $\begin{array}{l}44 \\
{[27-57]}\end{array}$ & $\mathrm{N} / \mathrm{A}$ \\
\hline $\begin{array}{l}\text { EASI } \\
\text { Median [IQR] }\end{array}$ & $\begin{array}{l}14.9 \\
{[10.6-18.5]}\end{array}$ & $\begin{array}{l}6.1 \\
{[4.8-9.5]}\end{array}$ & $\begin{array}{l}3.5 \\
{[2.7-5.1]}\end{array}$ & 0,025 \\
\hline $\begin{array}{l}\text { IGA } \\
\text { Median [IQR] }\end{array}$ & $\begin{array}{l}3 \\
{[3-4]}\end{array}$ & $\begin{array}{l}2 \\
{[2-2]}\end{array}$ & $\begin{array}{l}2 \\
{[1-2]}\end{array}$ & 0,032 \\
\hline $\begin{array}{l}\text { Eosinophil number } \\
\text { (x106 cells/ml) } \\
\text { Median [IQR] }\end{array}$ & $\begin{array}{l}0.33 \\
{[0.28-0.54]}\end{array}$ & $\begin{array}{l}0.49 \\
{[0.36-0.68]}\end{array}$ & $\begin{array}{l}0.37 \\
{[0.36-0.68]}\end{array}$ & 0,70 \\
\hline $\begin{array}{l}\text { Diagnosed with asthma } \\
(\mathrm{N}, \%)\end{array}$ & $10(62,5 \%)$ & $10(62,5 \%)$ & $8(57.1 \%)$ & N/A \\
\hline $\begin{array}{l}\text { Concomitant systemic } \\
\text { immunosuppressive } \\
\text { treatment }(\mathrm{N}, \%)\end{array}$ & $4(25.0 \%)$ & $4(25.0 \%)$ & $4(28.6 \%)$ & $\mathrm{N} / \mathrm{A}$ \\
\hline
\end{tabular}

Table 1. Patient characteristics. 


\section{Eosinophil activation in peripheral blood of AD patients was not affected by treatment with dupilumab}

At baseline, the expression of several activation markers, ${ }^{12,13}$ on eosinophils of our 16 enrolled patients was compared to a cohort of 9 healthy volunteers. The expression of all tested markers was significantly higher (CD66b, CD35 and CD11b) or lower (CD62L) in $A D$ patients compared to healthy volunteers (Figure 2 ). This fits very well with the finding that primed eosinophils are also found in the peripheral blood of patients with other T2-mediated diseases. This enhanced activation status of eosinophils is also supported by our finding that eosinophils from AD patients are less reactive to fMLF compared to healthy volunteers (Figure 3 and Supplementary Figure 2).This partial refractoriness of eosinophils in T2-disease has also been found in asthma patients ${ }^{13,14}$. Both findings are in line with previous studies showing a correlation in serum-ECP levels and disease severity in atopic dermatitis ${ }^{15,16}$. However, ECP measured in serum has its limitation because its levels are quite variable and are dependent on multiple ex vivo variables ${ }^{17}$.

A

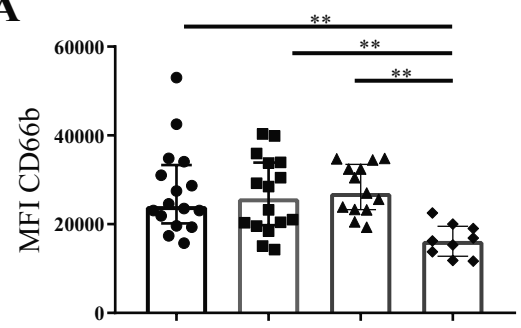

C
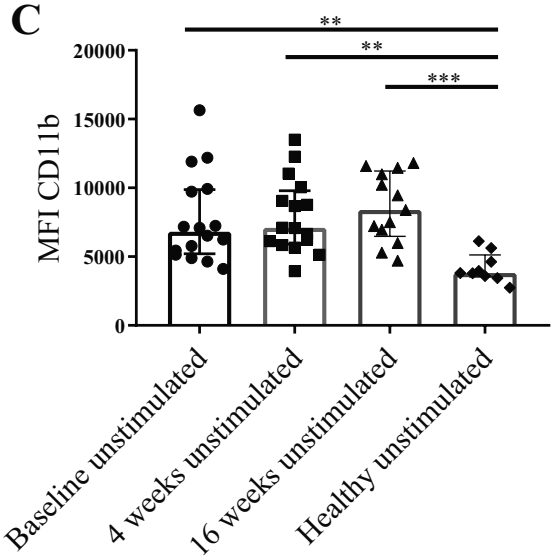

B

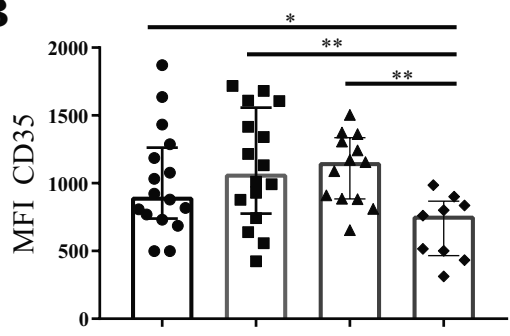

D

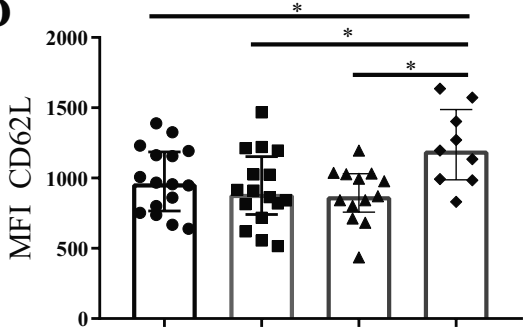

Figure 2. Comparison of activation markers on peripheral blood eosinophils.

The median fluorescence intensity (MFI) of CD66b (A), CD35 (B), CD11b (C) and CD62L(D) is plotted for $A D$ patients at baseline ( $n=16$, circles), after 4 weeks of treatment ( $n=16$, squares) and after 16 weeks of treatment ( $n=13$, triangles) with dupilumab. The MFI is compared to surface markers on eosinophils from healthy volunteers ( $n=9$, diamonds). The individual data points with the median and interquartile range are shown. A Friedman test (to compare the different treatment phases) or a Kruskal-Wallis test (to compare healthy volunteers to AD patients) was performed for statistical analysis. All groups were compared but only statistically significant results are indicated. * $P \leq 0.05, * * P \leq 0.01$ and $* * * P \leq 0.001$. 
A

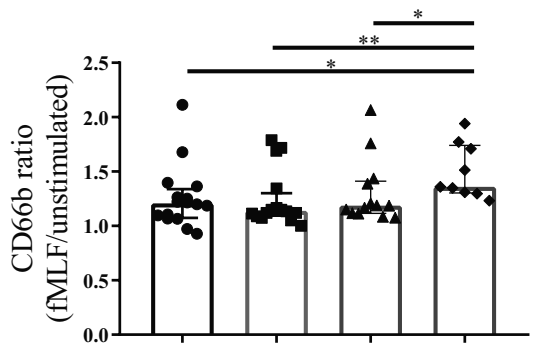

C

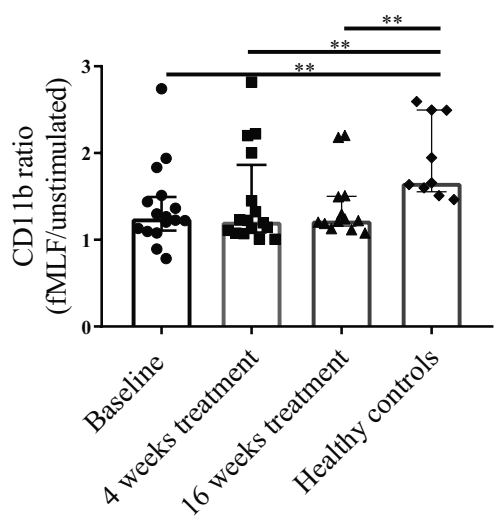

B

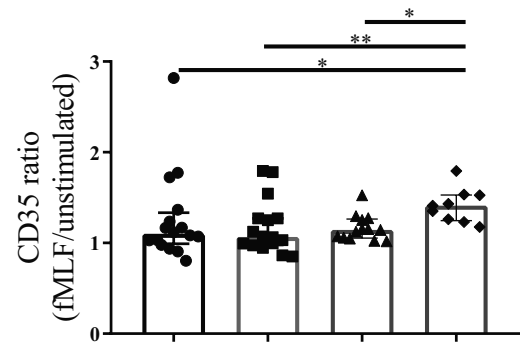

D

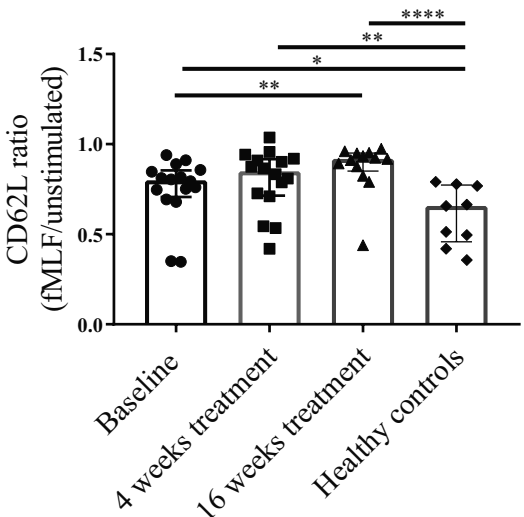

Figure 3. Comparison of degranulation potency on peripheral blood eosinophils.

The ratio between the MFI of $1 \mu \mathrm{M}$ fMLF stimulated and unstimulated eosinophils was calculated for CD66b (A), CD35 (B), CD11b (C) and CD62L(D). This is plotted for AD patients at baseline ( $n=16$, circles), after 4 weeks of treatment ( $n=16$, squares) and after 16 weeks of treatment ( $n=13$, triangles) with dupilumab. This ratio is also compared to samples taken from healthy volunteers ( $n=9$, diamonds). The individual data points with the median and interquartile range are shown. A Friedman test (to compare the different treatment phases) or a Kruskal-Wallis test (to compare healthy volunteers to AD patients) was performed for statistical analysis. All groups were compared but only statistically significant results are indicated. $* \mathrm{P} \leq 0.05, * * \mathrm{P} \leq 0.01$ and $* * * \mathrm{P} \leq 0.0001$.

We next studied eosinophils in AD patients after 4 weeks and 16 weeks of treatment with dupilumab. Eosinophil activation characterized by the increase of these activation markers was not affected by 4 or 16 weeks of treatment with dupilumab. These findings indicate that IL-4 and/or IL-13 are not involved in the (pre)activated state of eosinophils in the peripheral blood of AD patients.

In contrast to eosinophils, our data of the same surface activation markers on neutrophils indicated that these cells showed no signs of direct activation in AD, as we did not find any significant differences between $A D$ patients and healthy volunteers 
(Supplementary Figure 3). This finding is also in line with previous work ${ }^{18}$ showing that deposition of neutrophil specific (granule) proteins was absent in lesional skin of AD patients.

On the other hand, our data are consistent for the presence of less primed neutrophils in the peripheral blood of AD patients as the induction of degranulation associated markers on neutrophils induced by fMLF was slightly but significantly decreased in AD patients compared to healthy volunteers (Supplementary Figure 4C and 4D). This might be due to the effect of homing of the most primed neutrophils to damaged tissues, leaving behind the less primed neutrophils, similarly to eosinophils in asthma ${ }^{13}$. However, since $A D$ is associated with neutrophil paucity in the skin, the clinical significance of this finding remains uncertain ${ }^{19}$. Another explanation could be that neutrophils in AD are less responsive to $\mathrm{fMLF}$ as an effect of systemic IL- $4^{19}$. Supportive of this hypothesis is the finding that treatment with dupilumab after 16 weeks increased the ratio of $\mathrm{fMLF}$ stimulated over unstimulated expression of CD11b and to a lesser extent of CD35 (Supplementary Figure 4A and $4 B$ ), which suggests that neutrophil reactivity increases. More research is needed to further clarify this.

\section{Increased expression of CCR3 and CD44 on eosinophils after treatment with dupilumab}

The expression of CD193 (or C-C chemokine receptor 3/CCR3) on eosinophils in AD patients was not different from healthy volunteers. However, after 4 weeks of treatment with dupilumab, the expression of this receptor was significantly increased in $A D$ patients (Figure 4A). Surprisingly, after 16 weeks of treatment with dupilumab CD193 expression decreased. It is tempting to speculate that this initial increase of CD193 expression was the result of a reduction of recruitment of eosinophils to the skin, which is normally regulated by local production of eotaxins. The production of eotaxins by endothelial cells in the skin is orchestrated via IL-13 and to a lesser extent via IL- $4^{20}$. A blockade of IL-4 and IL-13 signaling might lead to a decrease of the systemic and local eotaxin production as was shown before ${ }^{10,11}$. Attenuation of CD193 expression might also be a result of eotaxin- 1 induced desensitization as has been described before in vitro $^{21}$. So it is conceivable that that a reduction of eotaxins might lead to an increase of CD193 expression. A similar association between low chemokine concentration and high chemokine receptor expression was described in neutrophils with regard to CXCL12 and CXCR4 respectively ${ }^{22}$. Alternatively, it is possible that blood eosinophils are overall more mature/senescent after treatment with dupilumab. We have previously shown that in homeostasis, CD193 is a maturation marker on eosinophils ${ }^{23}$. So, a lack of homing of circulatory eosinophils might lead to aging of eosinophils in the circulation which will lead to an increase of CD193 expression. 
A

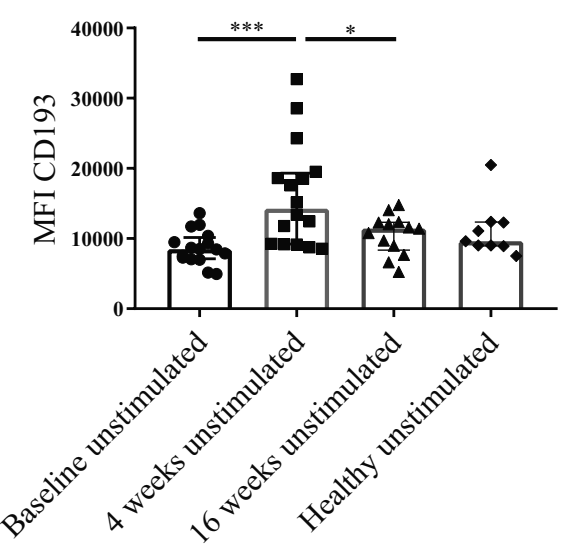

B
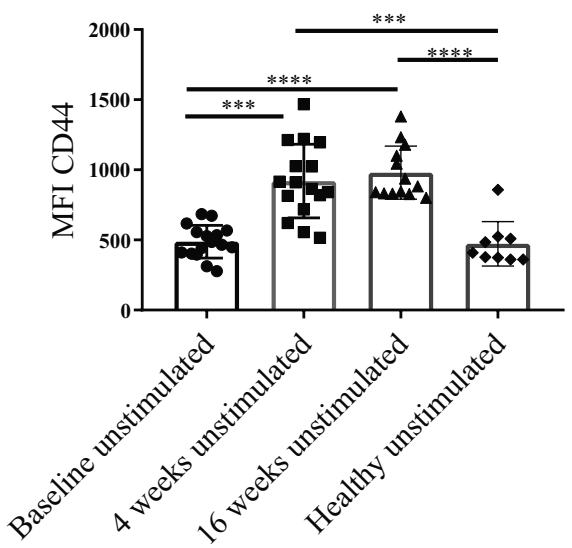

Figure 4. Comparison of surface level of CD193 and CD44 on peripheral blood eosinophils

The median fluorescence intensity (MFI) of CD193 (A) and CD44 (B) is plotted for AD patients at baseline ( $n=16$, circles), after 4 weeks of treatment ( $n=16$, squares) and after 16 weeks of treatment ( $n=13$, triangles) with dupilumab. The MFI is compared to surface markers on eosinophils from healthy volunteers ( $n=9$, diamonds). The individual data points with the median and interquartile range are shown. A Friedman test (to compare the different treatment phases) or a Kruskal-Wallis test (to compare healthy volunteers to $A D$ patients) was performed for statistical analysis. All groups were compared but only statistically significant results are indicated. $* \mathrm{P} \leq 0.05, * * * \mathrm{P} \leq 0.001$ and $* * * * \mathrm{P} \leq 0.0001$.

After 16 weeks of treatment with dupilumab, CD193 expression on eosinophils normalized. As we have shown previously, eotaxin-1 levels also returned to normal after longer term treatment with dupilumab ${ }^{10}$. Therefore, the lower CD193 expression might be the result of enhanced eotaxin-1 level. The mechanism(s) underlying the increase in eotaxin-1 after longer treatment is not known, but it may be that endothelial cells in the skin return to produce more eotaxin-1 as a result of compensation by other cytokines, such as IL5 , that are not downregulated by dupilumab ${ }^{10}$. However, IL-5 is not known to induce eotaxin production ${ }^{24}$. An alternative explanation as to why CD193 expression went down, is that the production of other chemokines (such as RANTES/CCL5) is compensating for the decrease in eotaxin production. These might in turn affect the expression of CD193. Little is known regarding this putative compensatory mechanism. It is unknown whether RANTES is affected by treatment with dupilumab, but it is known that the production of RANTES is increased in the skin of patients with $A D^{25}$. So in future studies level of Eotaxin and RANTES should be measured in the skin after 4 weeks and 16 weeks of treatment with dupilumab to be able to elucidate the underlying mechanism(s).

CD44 expression on eosinophils also increased during treatment with dupilumab (Figure 4B). In contrast to CD193, the increase of CD44 was persistent during 16 weeks of treatment. CD44 is a proteoglycan expressed on all leukocytes ${ }^{26}$. This receptor is possibly involved in cell trafficking, where it mediates rolling and adhesion to hyaluronic acid ${ }^{26}$. 
For eosinophils CD44 is known as an activation marker ${ }^{27}$. In asthma, CD44 is mainly expressed on sputum eosinophils, supporting the concept that CD44 facilitates homing of eosinophils. Interestingly, the expression of CD44 on peripheral blood eosinophils is higher in asthma patients that are well-controlled opposed to poorly controlled patients ${ }^{27}$. This suggests that CD44 high eosinophils are more likely to transmigrate to the tissue. When transmigration to the tissue is inhibited as a result of treatment with dupilumab, it is not unexpected that this will lead to an increase of the median CD44 expression on eosinophils ${ }^{13}$. CD44 expression remained high after longer treatment with dupilumab (16 weeks) which suggests that homing of eosinophils to the skin is still reduced after 16 weeks. This was confirmed in an earlier study performed by the group of Gutmann and her colleagues, in which major basis protein (MBP) infiltrates were reduced in the skin of AD patients after 16 weeks of treatment with dupilumab ${ }^{28}$. It should, however, be emphasized that in this study, a similar decrease of MBP was found in the placebo group. So, an additional study should be performed in order to elucidate this putative mechanism.

So far, the literature does not report many significant adverse events, associated with dupilumab induced eosinophilia ${ }^{29}$. There are, however, a few reports that mention tissue damage, caused by eosinophilia as a result of treatment with dupilumab ${ }^{30,31}$. Nonetheless, because grade 3 (severe) eosinophilia is very rare $(<1 \%)$ routine laboratory monitoring of eosinophil counts in clinical practice is not advised ${ }^{29}$.

Since, we have shown that patients with $A D$ do have primed and/or activated eosinophils, severe eosinophilia ( $\geq 1.5 \times 10^{6} \mathrm{cells} / \mathrm{ml}$ ) might (eventually) lead to increased health hazards $\mathrm{s}^{32,33}$. Therefore, it is perhaps sensible to monitor eosinophil counts during treatment with dupilumab, at least at the start of treatment.

\section{In conclusion}

Our data support the concept that treatment with dupilumab decreases eosinophil trafficking to the skin, by decreasing local production of eotaxins. This is supported by the decreased plasma levels of Eotaxin-1, Eotaxin-3, the increased expression of CD193 and the increased CD44 expression on eosinophils. Furthermore, peripheral blood eosinophils of $A D$ patients show an elevated activation state compared to healthy volunteers. This state is not altered after treatment with dupilumab. Since dupilumab can lead to an increase of peripheral blood eosinophils in certain patients, these eosinophils can possible mediate collateral damage to healthy tissues. Therefore, it might be prudent to monitor eosinophil counts and eosinophil (pre)activation during treatment of patients suffering from T2-diseases with dupilumab. 


\section{References}

1. Meeusen EN, Balic A. Do eosinophils have a role in the killing of helminth parasites? Parasitol Today [Internet]. 2000 [cited 2018 Jul 27];16:95-101. Available from: http://www.ncbi.nlm. nih.gov/pubmed/10689327

2. Marichal T, Mesnil C, Bureau F. Homeostatic Eosinophils: Characteristics and Functions. Front Med [Internet]. 2017 [cited 2018 Oct 8];4:101. Available from: http://www.ncbi.nlm. nih.gov/pubmed/28744457

3. Jacobsen EA, Helmers RA, Lee JJ, Lee NA. The expanding role(s) of eosinophils in health and disease. Blood [Internet]. 2012 [cited $2018 \mathrm{Jul}$ 27];120:3882-90. Available from: http:// www.ncbi.nlm.nih.gov/pubmed/22936660

4. Sullivan M, Silverberg NB. Current and emerging concepts in atopic dermatitis pathogenesis. Clin Dermatol [Internet]. 2017 [cited 2019 Apr 19];35:349-53. Available from: http://www. ncbi.nlm.nih.gov/pubmed/28709564

5. Kiehl P, Falkenberg K, Vogelbruch M, Kapp A. Tissue eosinophilia in acute and chronic atopic dermatitis: a morphometric approach using quantitative image analysis of immunostaining. Br J Dermatol [Internet]. 2001 [cited 2019 Mar 29];145:720-9. Available from: http://www. ncbi.nlm.nih.gov/pubmed/11736895

6. Leiferman KM, Ackerman SJ, Sampson HA, Haugen HS, Venencie PY, Gleich GJ. Dermal Deposition of Eosinophil-Granule Major Basic Protein in Atopic Dermatitis. N Engl J Med [Internet]. 1985 [cited 2019 Mar 29];313:282-5. Available from: http://www.nejm.org/doi/ abs/10.1056/NEJM198508013130502

7. Oldhoff JM, Darsow U, Werfel T, Katzer K, Wulf A, Laifaoui J, et al. Anti-IL-5 recombinant humanized monoclonal antibody (Mepolizumab) for the treatment of atopic dermatitis. Allergy [Internet]. 2005 [cited 2019 Apr 19];60:693-6. Available from: http://www.ncbi. nlm.nih.gov/pubmed/15813818

8. Simpson EL, Bieber T, Guttman-Yassky E, Beck LA, Blauvelt A, Cork MJ, et al. Two Phase 3 Trials of Dupilumab versus Placebo in Atopic Dermatitis. N Engl J Med [Internet]. 2016 [cited 2019 Apr 19];375:2335-48. Available from: http://www.ncbi.nlm.nih.gov/pubmed/27690741

9. Wenzel S, Castro M, Corren J, Maspero J, Wang L, Zhang B, et al. Dupilumab efficacy and safety in adults with uncontrolled persistent asthma despite use of medium-to-highdose inhaled corticosteroids plus a long-acting beta2 agonist: a randomised double-blind placebo-controlled pivotal phase $2 \mathrm{~b}$ dose-ranging trial. Lancet [Internet]. 2016;388:31-44. Available from: http://www.ncbi.nlm.nih.gov/pubmed/27130691

10. Ariëns LF, van der Schaft J, Bakker DS, Balak D, Romeijn M LE, Kouwenhoven T, et al. Dupilumab is very effective in a large cohort of difficult-to-treat adult atopic dermatitis patients: First clinical and biomarker results from the BioDay registry. Allergy [Internet]. 2019 [cited 2019 Oct 11];all.14080. Available from: http://www.ncbi.nlm.nih.gov/pubmed/31593343

11. Jonstam K, Swanson BN, Mannent LP, Cardell L-O, Tian N, Wang Y, et al. Dupilumab reduces local type 2 pro-inflammatory biomarkers in chronic rhinosinusitis with nasal polyposis. Allergy [Internet]. 2019 [cited 2019 Mar 29]; Available from: http://www.ncbi.nlm.nih.gov/ pubmed/30488542

12. R, Spijkerman L, Hesselink P, Hellebrekers N, Vrisekoop F H, LPH, Leenen L K. Automated Flow Cytometry Enables High Performance Point-Of-Care Analysis of Leukocyte Phenotypes. J Immunol Methods [Internet]. 2019 [cited 2019 Dec 12];474. Available from: https://pubmed. ncbi.nlm.nih.gov/31419409-automated-flow-cytometry-enables-high-performance-pointof-care-analysis-of-leukocyte-phenotypes/ 
13. Johansson MW. Activation states of blood eosinophils in asthma. Clin Exp Allergy [Internet]. 2014 [cited 2019 Oct 2];44:482-98. Available from: http://doi.wiley.com/10.1111/cea.12292

14. Luijk B, Lindemans CA, Kanters D, van der Heijde R, Bertics P, Lammers J-WJ, et al. Gradual increase in priming of human eosinophils during extravasation from peripheral blood to the airways in response to allergen challenge. J Allergy Clin Immunol [Internet]. 2005 [cited 2020 Mar 25];115:997-1003. Available from: http://www.ncbi.nlm.nih.gov/pubmed/15867857

15. Czech W, Krutmann J, Schöpf E, Kapp A. Serum eosinophil cationic protein (ECP) is a sensitive measure for disease activity in atopic dermatitis. Br J Dermatol [Internet]. 1992 [cited 2020 Jan 20];126:351-5. Available from: http://www.ncbi.nlm.nih.gov/pubmed/1571256

16. Pucci N, Lombardi E, Novembre E, Farina S, Bernardini R, Rossi E, et al. Urinary eosinophil protein $\mathrm{X}$ and serum eosinophil cationic protein in infants and young children with atopic dermatitis: correlation with disease activity. J Allergy Clin Immunol [Internet]. 2000 [cited 2020 Jan 20];105:353-7. Available from: http://www.ncbi.nlm.nih.gov/pubmed/10669858

17. Pronk-Admiraal CJ, Bartels PC. Total amount of ECP per eosinophil as indicator for the activity state of eosinophils. Scand J Clin Lab Invest [Internet]. 2001 [cited 2020 Jan 20];61:453-7. Available from: http://www.ncbi.nlm.nih.gov/pubmed/11681535

18. Ott NL, Gleich GJ, Peterson EA, Fujisawa T, Sur S, Leiferman KM. Assessment of eosinophil and neutrophil participation in atopic dermatitis: comparison with the IgE-mediated late-phase reaction. J Allergy Clin Immunol [Internet]. 1994 [cited 2020 Jan 20];94:120-8. Available from: http://www.ncbi.nlm.nih.gov/pubmed/8027490

19. Woytschak J, Keller N, Krieg C, Impellizzieri D, Thompson RW, Wynn TA, et al. Type 2 Interleukin-4 Receptor Signaling in Neutrophils Antagonizes Their Expansion and Migration during Infection and Inflammation. Immunity [Internet]. 2016 [cited 2020 Jan 20];45:172-84. Available from: http://www.ncbi.nlm.nih.gov/pubmed/27438770

20. Kagami S, Saeki H, Komine M, Kakinuma T, Tsunemi Y, Nakamura K, et al. Interleukin-4 and interleukin-13 enhance CCL26 production in a human keratinocyte cell line, HaCaT cells. Clin Exp Immunol [Internet]. 2005 [cited 2020 Jan 24];141:459-66. Available from: http://www. ncbi.nlm.nih.gov/pubmed/16045735

21. Dulkys Y, Kluthe C, Buschermöhle T, Barg I, Knöss S, Kapp A, et al. IL-3 induces down-regulation of CCR3 protein and mRNA in human eosinophils. J Immunol [Internet]. 2001 [cited $2018 \mathrm{Sep}$ 25];167:3443-53. Available from: http://www.ncbi.nlm.nih.gov/pubmed/11544337

22. Martin C, Burdon PCE, Bridger G, Gutierrez-Ramos JC, Williams TJ, Rankin SM. Chemokines acting via CXCR2 and CXCR4 control the release of neutrophils from the bone marrow and their return following senescence. Immunity [Internet]. 2003 [cited 2019 Nov 21];19:583-93. Available from: http://www.ncbi.nlm.nih.gov/pubmed/14563322

23. Hassani M, van Staveren S, van Grinsven E, Bartels M, Tesselaar K, Leijte G, et al. Characterization of the phenotype of human eosinophils and their progenitors in the bone marrow of healthy individuals. Haematologica [Internet]. 2019 [cited 2019 Sep 19]; haematol.2019.219048. Available from: http://www.ncbi.nlm.nih.gov/pubmed/31101758

24. Humbles AA, Conroy DM, Marleau S, Rankin SM, Palframan RT, Proudfoot AE, et al. Kinetics of eotaxin generation and its relationship to eosinophil accumulation in allergic airways disease: analysis in a guinea pig model in vivo. J Exp Med [Internet]. 1997 [cited 2020 Jan 23];186:60112. Available from: http://www.ncbi.nlm.nih.gov/pubmed/9254658

25. Kaburagi Y, Shimada Y, Nagaoka T, Hasegawa M, Takehara K, Sato S. Enhanced production of CC-chemokines (RANTES, MCP-1, MIP-1alpha, MIP-1beta, and eotaxin) in patients with atopic dermatitis. Arch Dermatol Res [Internet]. 2001 [cited 2020 Jan 23];293:350-5. Available from: http://www.ncbi.nlm.nih.gov/pubmed/11550808 
26. Matsumoto K, Appiah-Pippim J, Schleimer RP, Bickel CA, Beck LA, Bochner BS. CD44 and CD69 represent different types of cell-surface activation markers for human eosinophils. Am J Respir Cell Mol Biol [Internet]. 1998 [cited 2020 Jan 23];18:860-6. Available from: http:// www.ncbi.nlm.nih.gov/pubmed/9618391

27. Sano K, Yamauchi K, Hoshi H, Honma M, Tamura G, Shirato K. CD44 expression on blood eosinophils is a novel marker of bronchial asthma. Int Arch Allergy Immunol [Internet]. 1997 [cited 2020 Jan 23];114 Suppl 1:67-71. Available from: http://www.ncbi.nlm.nih.gov/ pubmed/9363930

28. Guttman-Yassky E, Bissonnette R, Ungar B, Suárez-Fariñas $M$, Ardeleanu M, Esaki $H$, et al. Dupilumab progressively improves systemic and cutaneous abnormalities in patients with atopic dermatitis. J Allergy Clin Immunol [Internet]. 2019 [cited 2020 Mar 30];143:155-72. Available from: http://www.ncbi.nlm.nih.gov/pubmed/30194992

29. Wollenberg A, Beck LA, Blauvelt A, Simpson EL, Chen Z, Chen Q, et al. Laboratory safety of dupilumab in moderate-to-severe atopic dermatitis: results from three phase III trials (LIBERTY AD SOLO 1, LIBERTY AD SOLO 2, LIBERTY AD CHRONOS). Br J Dermatol [Internet]. 2019 [cited 2020 Mar 30];bjd.18434. Available from: http://www.ncbi.nlm.nih.gov/ pubmed/31407311

30. Menzella F, Montanari G, Patricelli G, Cavazza A, Galeone C, Ruggiero P, et al. A case of chronic eosinophilic pneumonia in a patient treated with dupilumab. Ther Clin Risk Manag [Internet]. 2019 [cited 2020 Mar 30];Volume 15:869-75. Available from: http://www.ncbi. nlm.nih.gov/pubmed/31371974

31. Deeks ED. Dupilumab: A Review in Moderate to Severe Asthma. Drugs [Internet]. 2019 [cited 2020 Mar 30];79:1885-95. Available from: http://www.ncbi.nlm.nih.gov/ pubmed/31728838

32. Ramírez E, Medrano-Casique N, Tong HY, Bellón T, Cabañas R, Fiandor A, et al. Eosinophilic drug reactions detected by a prospective pharmacovigilance programme in a tertiary hospital. Br J Clin Pharmacol [Internet]. 2017 [cited 2020 Mar 30];83:400-15. Available from: http://www.ncbi.nlm.nih.gov/pubmed/27543764

33. Roufosse F, Weller PF. Practical approach to the patient with hypereosinophilia. J Allergy Clin Immunol [Internet]. 2010 [cited 2020 Mar 30];126:39-44. Available from: https:// linkinghub.elsevier.com/retrieve/pii/S0091674910006603 

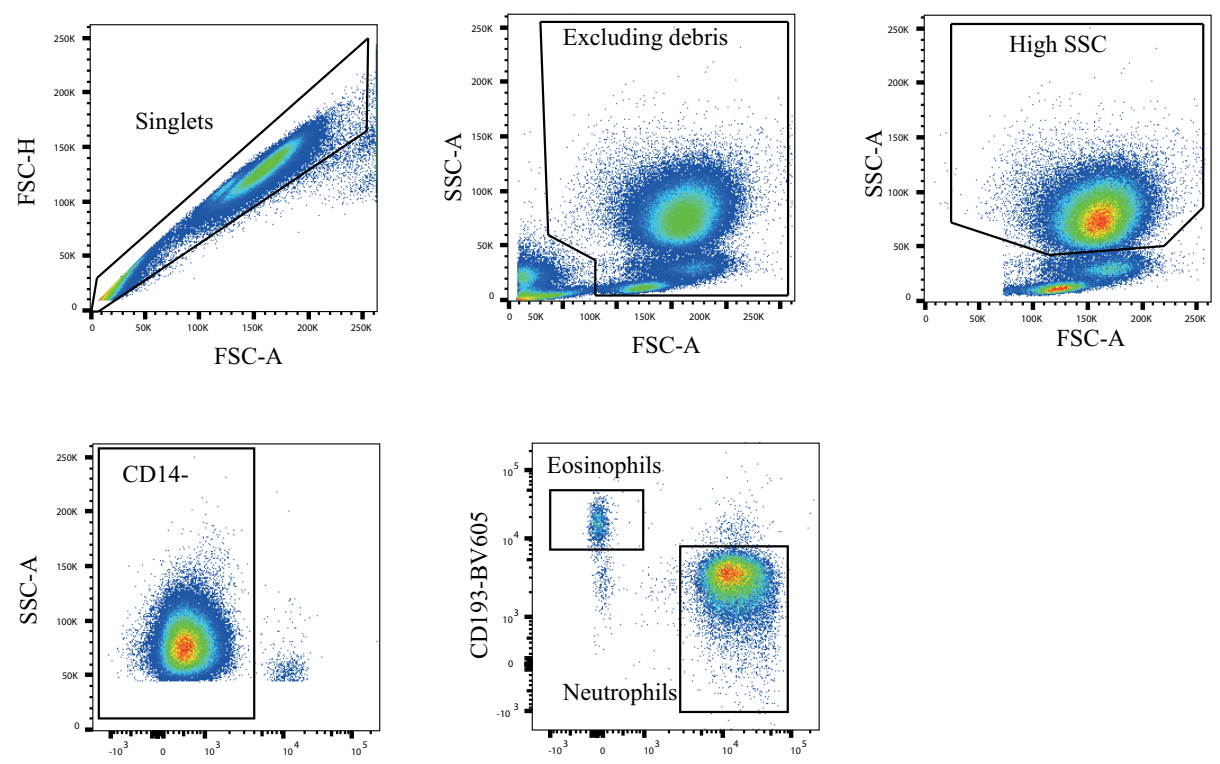

CD14-PB

CD16-BV785

Supplementary Figure 1. Gating strategy for neutrophils and eosinophils in peripheral blood.

The different steps to gate eosinophils and neutrophils are depicted. Cells were gated for singlets (A), debris was excluded (B), SSC high $(\mathbf{C})$, exclusion of CD14 high $(\mathbf{D})$ and finally eosinophils were gated as $\mathrm{CD} 16^{\text {neg }}$ and $C D 193^{\text {high }}$ and neutrophils were gated as CD16 ${ }^{\text {high }}$ and $C D 193^{\text {neg }}(E)$. 
A

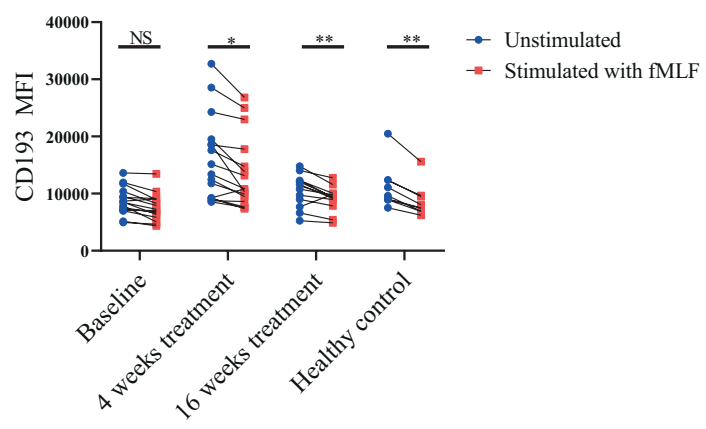

C

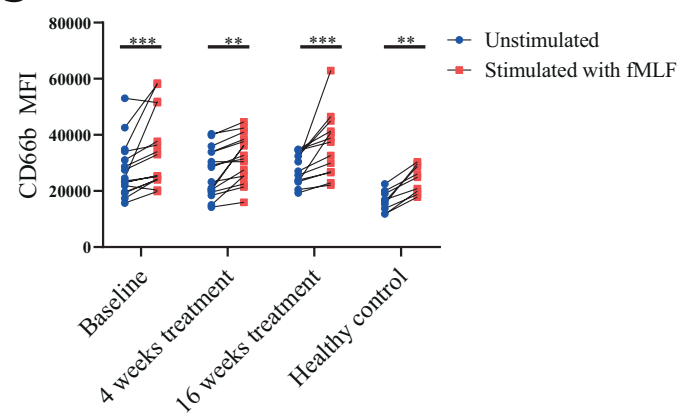

$\mathbf{E}$

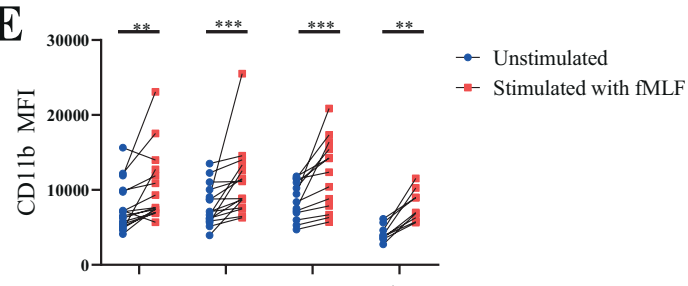

B

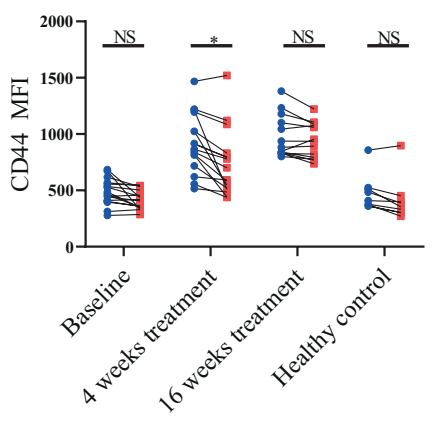

D

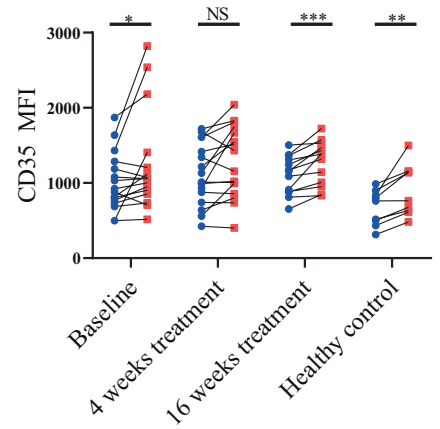

$\mathbf{F}$

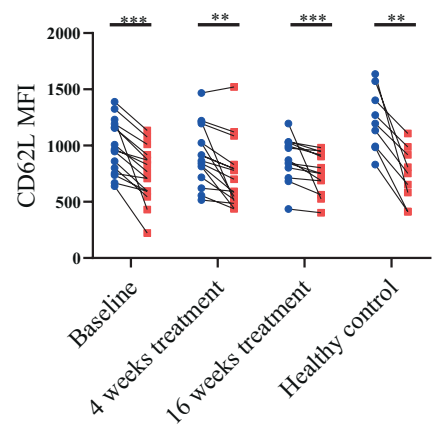

Supplementary Figure 2. Effect of fMLF on surface markers on eosinophils.

The median fluorescence intensity (MFI) is plotted for different surface markers for unstimulated (blue) and for $1 \mu \mathrm{M}$ fMLF stimulated (red) eosinophils at baseline ( $n=16)$, after 4 weeks of treatment with dupilumab ( $n=16)$, after 16 weeks of treatment with dupilumab $(n=13)$ and for healthy volunteers $(n=9)$. A. CD193, B. CD44, C. CD66b, D. CD35, E. CD11b and F. CD62L. A Mann-Withney test was performed for statistical analysis. NS. Not significant. ${ }^{*} \mathrm{P} \leq 0.05,{ }^{* *} \mathrm{P} \leq 0.01$ and ${ }^{* * *} \mathrm{P} \leq 0.001$. 
A

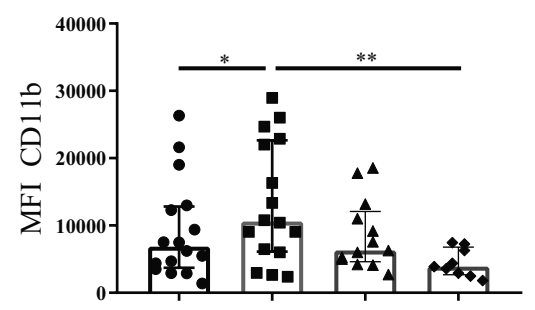

C

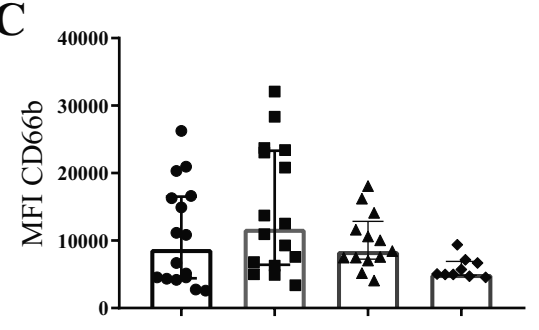

E

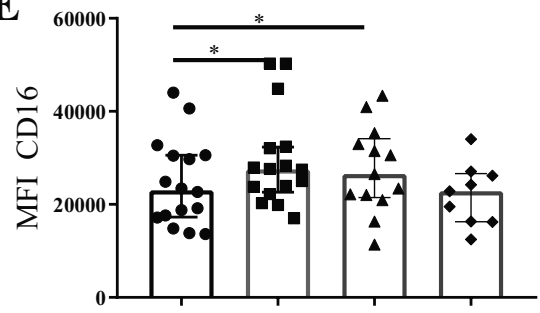

B

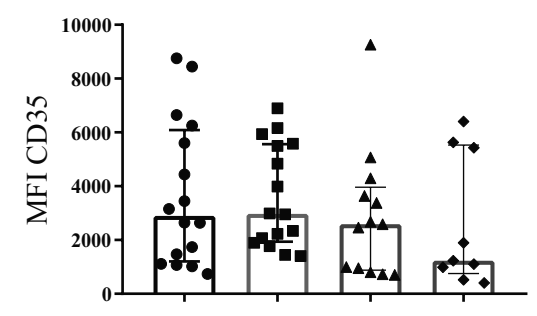

D
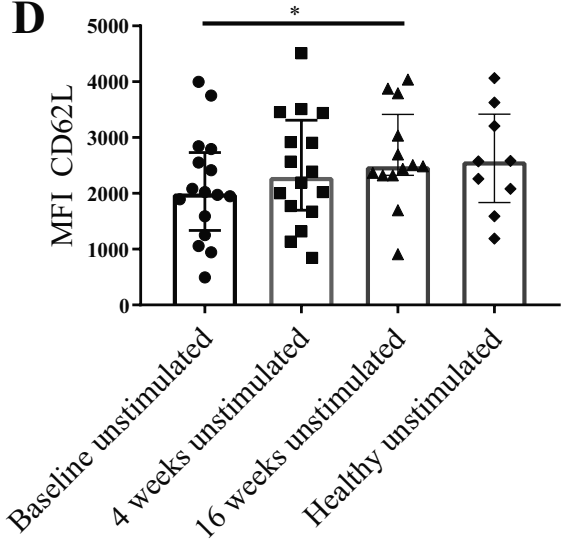

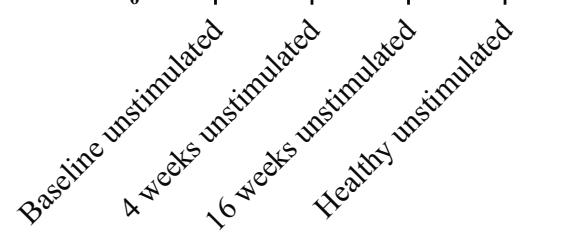

Supplementary Figure 3. Comparison of activation markers on peripheral blood neutrophils

The median fluorescence intensity (MFI) of CD66b (A), CD35 (B), CD11b (C), CD62L(D) and CD16 (E) is plotted for $A D$ patients at baseline ( $n=16$, circles), after 4 weeks of treatment ( $n=16$, squares) and after 16 weeks of treatment ( $n=13$, triangles) with dupilumab. The MFI is compared to surface markers on eosinophils from healthy volunteers ( $n=9$, diamonds). The individual data points with the median and interquartile range are shown. A Friedman test (to compare the different treatment phases) or a Kruskal-Wallis test (to compare healthy volunteers to AD patients) was performed for statistical analysis. All groups were compared but only statistically significant results are indicated. ${ }^{*} \mathrm{P} \leq 0.05$ and $* * \mathrm{P} \leq 0.01$. 
A

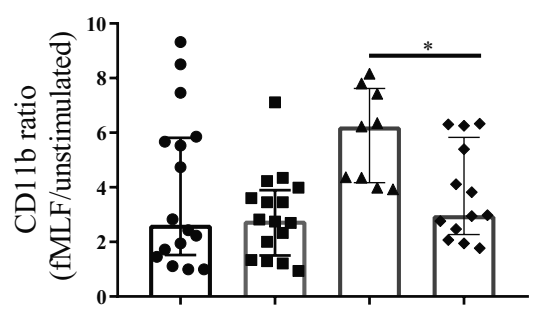

C

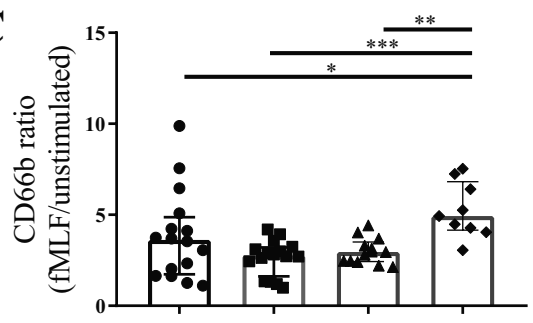

E

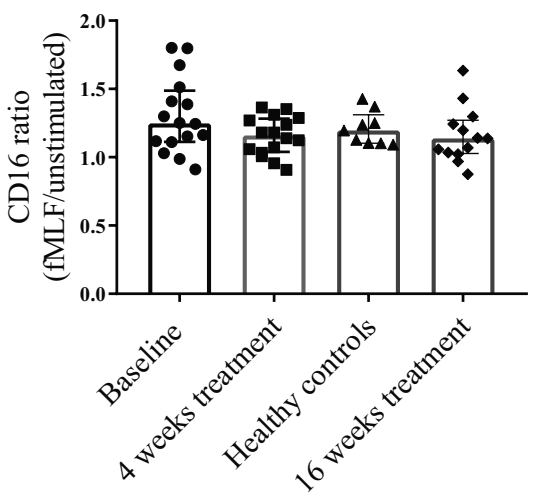

B
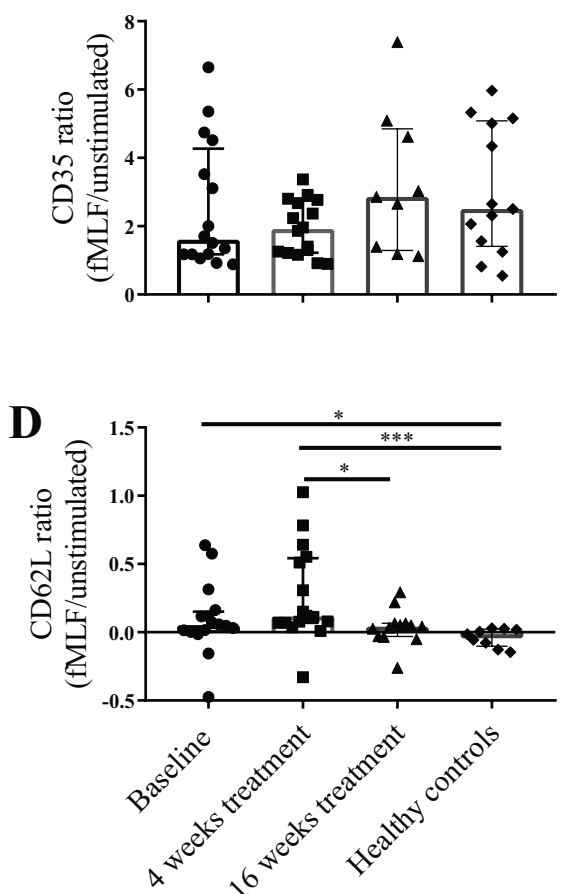

Supplementary Figure 4. Comparison of degranulation potency on peripheral blood neutrophils.

The ratio between the MFI of $1 \mu \mathrm{M}$ fMLF stimulated and unstimulated neutrophils was calculated for CD66b (A), CD35 (B), CD11b (C), CD62L (D) and CD16 (E). This is plotted for AD patients at baseline ( $\mathrm{n}=16$, circles), after 4 weeks of treatment ( $n=16$, squares) and after 16 weeks of treatment ( $n=13$, triangles) with dupilumab. This ratio is also compared to samples taken from healthy volunteers ( $n=9$, diamonds). The individual data points with the median and interquartile range are shown. A Friedman test (to compare the different treatment phases) or a Kruskal-Wallis test (to compare healthy volunteers to AD patients) was performed for statistical analysis. All groups were compared but only statistically significant results are indicated. * $\mathrm{P} \leq 0.05, * * \mathrm{P} \leq 0.01$ and $* * * \mathrm{P} \leq 0.001$. 
A
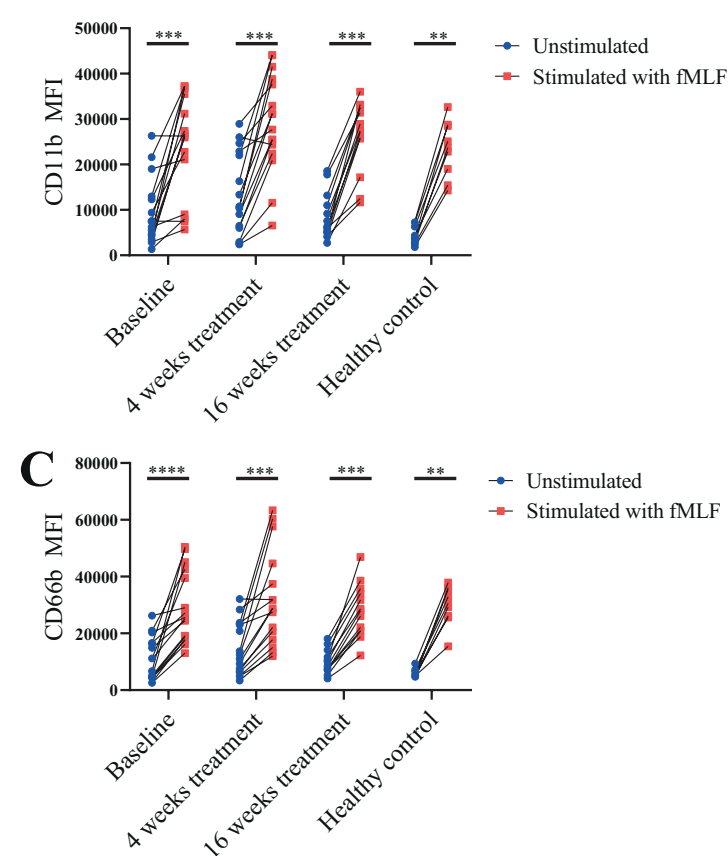

B
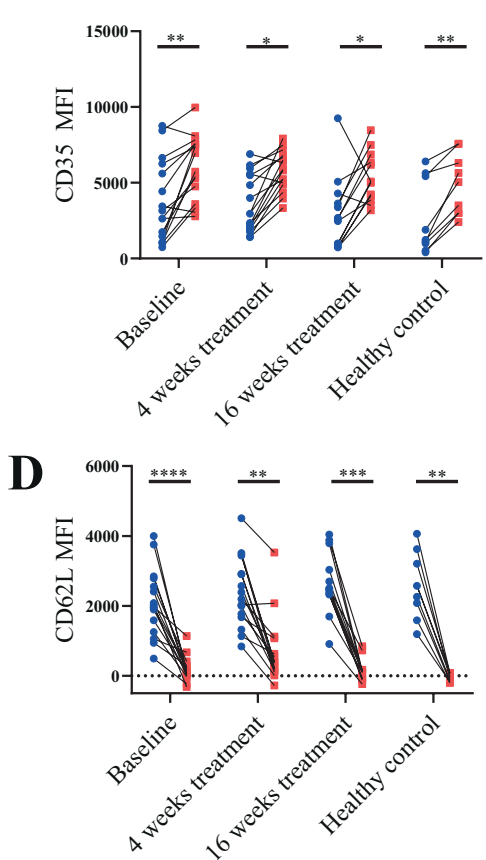

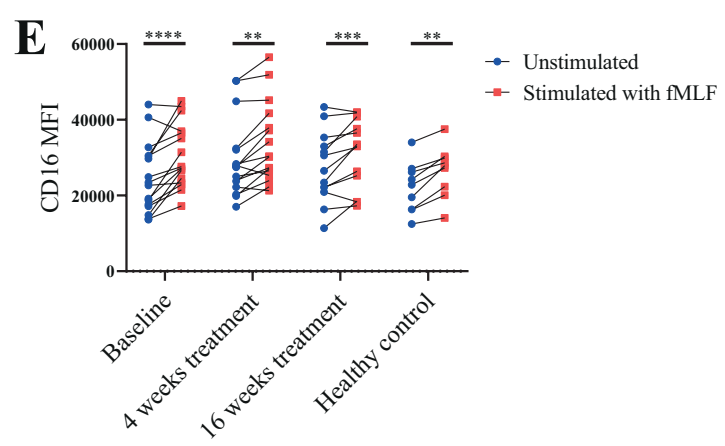

\section{Supplementary Figure 5. Effect of fMLF on surface markers on neutrophils}

The median fluorescence intensity (MFI) is plotted for different surface markers for unstimulated (blue) and for $1 \mu \mathrm{M}$ fMLF stimulated (red) neutrophils at baseline ( $n=16)$, after 4 weeks of treatment with dupilumab ( $n=16)$, after 16 weeks of treatment with dupilumab $(n=13)$ and for healthy volunteers $(n=9)$. A. CD11b, B. CD35, C. CD66b, D. CD62L and E. CD16. A Mann-Withney test was performed for statistical analysis. * $P \leq 0.05$, ** $P \leq 0.01, * * * P \leq 0.001$ and $* * * P \leq 0.0001$. 



\section{Chapter 11}

General discussion 
The content of this thesis is mainly focused on the description of granulocyte activation, maturation and kinetics in healthy humans and in patients with atopic diseases (mainly eosinophilic asthma). Granulocyte kinetics are determined by different factors: proliferation (number of cells in mitosis and mitosis time) of the granulocyte progenitors in the bone marrow; the time of maturation of the post mitotic progenitors after the last division (post mitotic transit time); the time after which (mature) granulocytes are released into the circulation; and finally the lifespan of granulocytes in the circulation or tissues (i.e. until (programmed) cell death). An easy, reliable and safe method to determine cell kinetics is labelling with $6,6-{ }^{2} \mathrm{H}_{2}-$ glucose. This label contains deuterium $\left({ }^{2} \mathrm{H}\right)$ which is administered orally every half hour during 5.5 hours. During this time ${ }^{2} \mathrm{H}$ will be incorporated in the DNA of dividing granulocyte progenitors during the S-phase of the cell cycle. This allows us to follow the pulse of incorporation of ${ }^{2} \mathrm{H}$ in the DNA in time by sampling the cells of interest on multiple days after label intake. We can finally use a combination of gas chromatography and mass spectrometry to detect ${ }^{2} \mathrm{H}$-enrichment in the DNA.

The first part of this thesis focused mainly on neutrophil activation, kinetics and signaling in healthy humans, while second part of this thesis focused on eosinophil differentiation, activation and kinetics in healthy humans and patients with eosinophilic asthma or atopic dermatitis.

In this chapter the results and conclusions of the previous chapters are described/ interpreted in more depth.

\section{Granulocyte compartment: the existence of multiple phenotypes:}

Neutrophil compartment: the idea that neutrophils consist of multiple phenotypes, has been accepted for several decades ${ }^{1}$. However, this heterogeneity has been described by multiple (and divergent) characteristics such as morphology, phenotype, function or a combination of those factors ${ }^{2}$. Our group recognized three heterogeneous neutrophil populations that we have addressed as three neutrophil "subsets": banded neutrophils (CD16 $\left.{ }^{\text {dim }} C D 62 L^{\text {high }}\right)$, mature neutrophils $\left(C D 16^{\text {high }} C D 62 L^{\text {high }}\right)$ and hypersegmented neutrophils $\left(C D 16^{\text {high }} \mathrm{CD} 6 \mathrm{~L}^{\mathrm{dim}}\right)^{3,4,5}$ of which two (banded and hypersegmented neutrophils) only appear in the blood in case of (acute) inflammation. These neutrophils were not only morphologically different, but we also acknowledged that banded neutrophils were far better in the containment of methicillin-resistant Staphylococcus aureus (MRSA) bacteria than hypersegmented neutrophils ${ }^{5}$. On the other hand, hypersegmented neutrophils were unique in their ability to suppress lymphocyte proliferation ${ }^{3}$.

In this thesis, (chapter 3 ) we have reviewed an additional source of neutrophil heterogeneity: neutrophil buoyant density. Although, it was already established that in certain conditions neutrophils co-segregate with mononuclear cells after densitygradient isolation ${ }^{6}$, we described the buoyant density of neutrophils as a continuous distribution, also during homeostasis. This "spectrum" of density in homeostasis is associated with differences in neutrophil functions, similarly to what is described in certain inflammatory diseases ${ }^{7,8}$. So low density neutrophils found in several chronic inflammatory diseases might be cells already present in homeostasis albeit with a 
somewhat higher density keeping them in a normal density population after density centrifugation.

In conclusion, our data imply that during acute or chronic inflammation certain neutrophil "subsets" appear in the blood such as banded and hypersegmented neutrophils ${ }^{9}$. In contrast, diseases can alter characteristics of homeostatic cells already present in blood. These pre-activated or primed cells can exhibit changed characteristics such as expression of surface markers and buoyant density.

The complexity of neutrophil heterogeneity is even further complicated by differences in production and kinetics. It is believed that neutrophils follow daily cycles of release from and migration back into the bone marrow ${ }^{10}$. So, the composition of neutrophils will be different, dependent on the time of the day ${ }^{11}$, which will also be reflected by differences in neutrophil function.

\section{Eosinophil compartment}

Regarding eosinophil heterogeneity much less is known. In 2016, Mesnil and her colleagues showed in a murine model of asthma that two types of eosinophil subsets exist ${ }^{12}$. They distinguished inflammatory eosinophils from "lung-resident eosinophils". The first "subset" is only recruited to the bronchial airways during (acute) inflammation caused by a challenge with house dust mite (a model for asthma). The latter are always present in the lung parenchyma and have an immune regulatory function ${ }^{12}$. The authors of this study have also discovered that in contrast to inflammatory eosinophils, lung-resident eosinophils are not affected by treatment with systemic anti-IL-5, making them IL-5 independent. Although, their findings were mostly established in mice, the data suggested that human lungs of deceased asthmatics also contained both eosinophil subsets. In humans, lung resident eosinophils were lower in IL-3R $\alpha$-expression and higher in CD62L-expression. Besides, in a human study, treatment with mepolizumab resulted in a decrease of IL-3R $\alpha$-expression and increase of CD62L-expression in peripheral blood eosinophils (chapter 9) ${ }^{13}$. This finding might be explained by a shift in abundance from inflammatory eosinophils before to resident eosinophils after treatment with anti-IL-5.

In chapter 9, we have confirmed that treatment with mepolizumab leads to a rapid and clear decrease of peripheral blood eosinophils (see Figure 2A of chapter 9). Although subtle, the remaining eosinophils indeed had lower IL-3R $\alpha$-expression and higher CD62L-expression. Therefore, it is possible that the remaining eosinophils after treatment with mepolizumab are enriched with these IL-5 independent resident eosinophils. However, after longer treatment with mepolizumab, the kinetics change back to "normal". This finding is not in line with the existence of a possible IL-5 independent subset, because it is not intuitive that long term treatment with mepolizumab would abolish its kinetics.

\section{Why is label in hypersegmented neutrophils delayed in asthma and not after endotoxemia?}

We have previously shown that human endotoxemia leads to the recruitment of hypersegmented neutrophils (CD16 ${ }^{\text {high }}$ CD62 $\mathrm{L}^{\text {dim }}$ neutrophils) to the peripheral blood ${ }^{3}$. We have also shown that these hypersegmented immune-suppressive neutrophils 
are most likely a different neutrophil subset, since they have similar kinetics to normal segmented neutrophils (CD16 ${ }^{\text {high }} C D 62 L^{\text {high }}$ neutrophils) while their proteomic profile is very distinct ${ }^{4}$. In the blood of eosinophilic asthmatics, hypersegmented neutrophils are also present, irrespective of treatment with mepolizumab or placebo (chapter 4). In contrast to endotoxemia, hypersegmented neutrophils are delayed around 1 day in their kinetics, compared to normal segmented neutrophils (chapter 4, Figure 4A). This difference between asthma and endotoxemia might be explained by two possible underlying mechanisms:

1. A simple explanation could be that (subtle) changes in kinetics between normal and hypersegmented neutrophils were missed in the study applying endotoxemia, because lower number of sampling points were taken. In addition, these sampling points were two days apart ${ }^{4}$. Indeed, after endotoxemia only 4 data points were collected on day 7 after label intake, while in asthmatics a total of 23 data points! were collected on day 7 after label intake. So the delay in label in hypersegmented neutrophils in the LPS study might have been missed. However, it is important to emphasize that the maximal enrichment was not different between the cell types suggesting that the peak was not missed.

2. Another explanation might be that endotoxemia changed neutrophil kinetics. Indeed, an LPS challenge in humans leads to an increase of G-CSF production and/ or release ${ }^{14}$, a cytokine known to be important in emergency granulopoiesis ${ }^{15}$. It is likely that this can affect the kinetics of the cells. This is supported by our finding in our unpublished work, that ${ }^{2} \mathrm{H}$-enrichment in neutrophil progenitors and mature neutrophils is accelerated after endotoxemia (Figure 1) compared to homeostasis (similarly as in chapter 2 ). Therefore, it is not unimaginable that the subtle differences in kinetics between normal segmented and hypersegmented neutrophils become even less apparent after accelerated granulopoiesis. In eosinophilic asthma such emergency neutrophiliopoiesis is not likely to be initiated. 


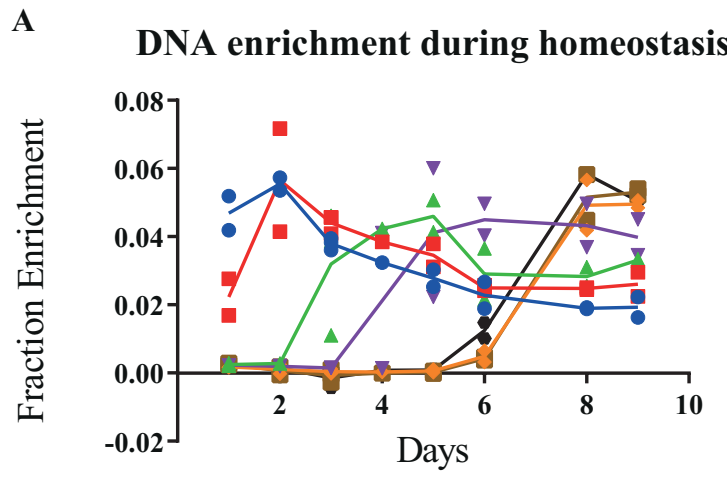

- Promyelocytes

$\rightarrow$ - Myelocytes

$₫$ Metamyelocytes

$\rightarrow$ Bandeds

$\rightarrow$ Mature neutrophils BM

- Mature neutrophils blood

$\rightarrow$ Oral neutrophils

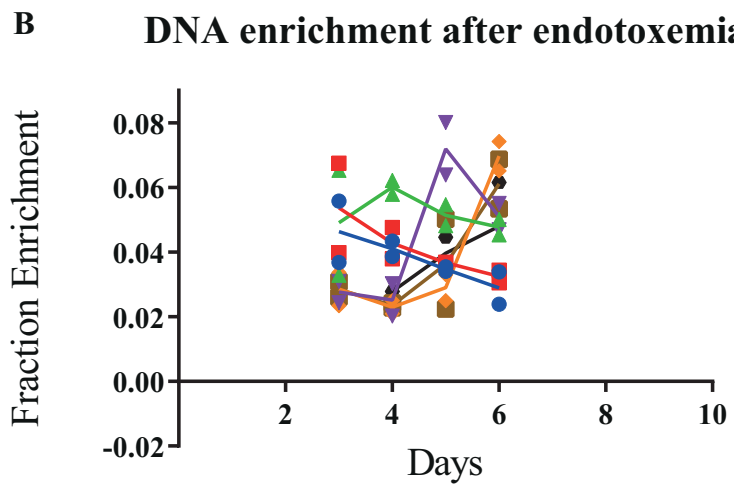

- Promyelocytes

-n Myelocytes

$\leftarrow$ Metamyelocytes

$\rightarrow$ Bandeds

$\rightarrow$ Mature neutrophils BM

- Oral neutrophils

$\rightarrow$ Oral neutrophils

Figure 1. Kinetics of neutrophils and their progenitors before and after endotoxemia.

The ratio of ${ }^{2} \mathrm{H}$-labelled and total DNA of neutrophils and their progenitors in the bone marrow, blood and the oral rinse were determined, similarly as in chapter $\mathbf{2}$ during homeostasis (A) and after human endotoxemia (B). After endotoxemia, especially kinetics of metamyelocytes and banded neutrophils are accelerated with one day.

\section{Are higher density neutrophils more mature than lower density neutrophils?} In chapter 3, we have shown that during homeostasis, neutrophils are heterogeneous in their buoyant densities and that these difference densities are also accompanied by functional differences. Lower density neutrophils (LDNs) are better in the suppression of lymphocyte proliferation, whereas the higher density neutrophils (HDNs) are less capable in bacterial containment (chapter 3). We have also shown that LDNs are enriched with banded neutrophils, especially after endotoxemia, which makes it likely that segmented neutrophils in the LDN fraction are relatively more immature compared to the segmented neutrophils in the HDN fraction. Indeed, after endotoxemia, we have also found that hypersegmented neutrophils ( $C D 16^{\text {high }} \mathrm{CD} 62 \mathrm{~L}^{\mathrm{dim}}$ neutrophils) are more abundant in the normal density fraction compared to the PBMC fraction after Ficoll-Paque centrifugation (Figure 2). Since, hypersegmented neutrophils are most likely more mature than normal segmented neutrophils (chapter 4 ) and banded neutrophils are more immature than normal segmented neutrophils (chapter 2$)^{4}$, neutrophil density is most likely intertwined with neutrophil maturation/ nuclear segmentation and functionality (Figure 2C). 
A

Banded neutrophils

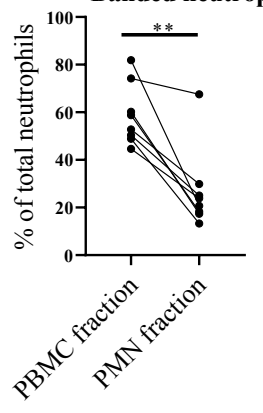

B

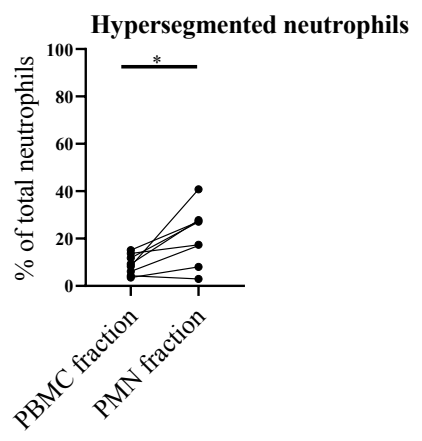

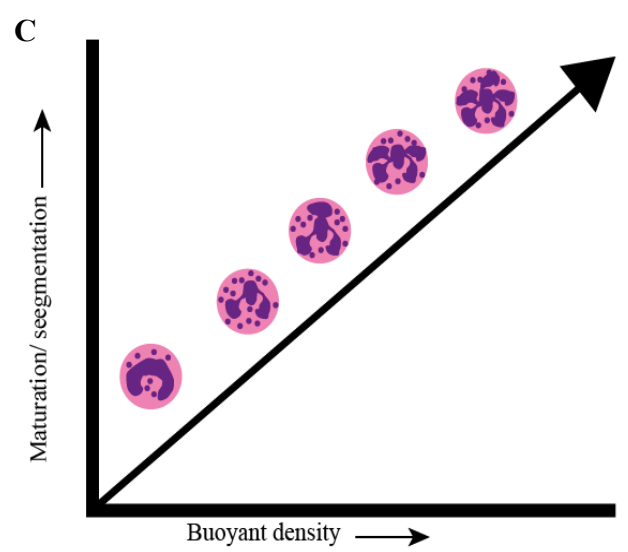

Figure 2. Correlation between nuclear segmentation and buoyant density in neutrophils.

The percentage of banded neutrophils (A) and hypersegmented neutrophils (B) was determined in the blood after endotoxemia and after Ficoll-Paque centrifugation in the PBMC and PMN fraction. Banded neutrophils are more abundant in the PBMC fraction, while hypersegmented neutrophils are more abundant in the PMN fraction. A schematic representation of the correlation between buoyant density and nuclear segmentation is shown in $\mathbf{C}$.

\section{Why are there neutrophil progenitors present in the circulation?}

In chapter 4, we have established that there are cells present in the circulation of asthmatics that are SSC ${ }^{\text {high }}, \mathrm{CD} 193^{\text {neg }}, \mathrm{CD} 14^{\text {neg }}, \mathrm{CD} 16^{\text {neg }}$ and $\mathrm{CD} 62 \mathrm{~L}^{\text {neg }}$. We sorted these cells and made cytospins that were additionally stained with May-Grünwald-Giemsa. Thereafter, we assessed these cells on the basis of nuclear morphology and granular content (see chapter $\mathbf{4}$ and Figure $3 \mathrm{~A}$ ) and discovered that they were morphologically very similar to neutrophil progenitors (myelocytes and promyelocytes, chapter 2) ${ }^{16}$. Also, when we assessed the kinetics of these cells in asthmatics, ${ }^{2} \mathrm{H}$-enrichment was at least 3 days earlier than mature neutrophils (chapter 4). Unfortunately, we had no data at day 1 and 2 after label intake, so it is possible that label is already present on 
those days. So, given both cell morphology and kinetics, these cells are undoubtedly neutrophil progenitors. Although in vitro differentiation assays are required to proof this hypothesis. Next, we wondered whether these neutrophil progenitors were also present in healthy humans. Therefore, by using the same gating strategy as was shown in chapter 4 , we determined the absolute count of blood neutrophil progenitors (CD16 ${ }^{\text {neg }} C D 62 L^{\text {neg }}$ ) in 12 male healthy volunteers (Figure $3 \mathrm{~B}$ ). We also determined the number of blood neutrophil progenitors in some of these volunteers $(n=7)$ after human endotoxemia, similarly as is explained in chapter 8. Indeed, both during homeostasis and after endotoxemia, the number of blood neutrophil progenitors are comparable to patients with (eosinophilic) asthma (Figure 3B). Next, we determined based on CD11b expression the percentage of myelocytes in these healthy volunteers (CD11bos, chapter 2 ) within the gated population of all neutrophil progenitors (combination of promyelocytes and myelocytes) and we identified that the far majority of blood neutrophil progenitors are myelocytes (approximately 83\%). Surprisingly, the percentage of myelocytes within the total neutrophil progenitors increased even more after endotoxemia to approximately 91\% (Figure 3C). So, the absolute number of all neutrophil progenitors did not change after, endotoxemia, but the number of myelocytes did increase. This might be the result of consumption of blood promyelocytes or the specific release of myelocytes from the bone marrow or another compartment.

Next, we compared the kinetics of the pooled blood neutrophil progenitors of the patients with asthma (chapter 4) to the kinetics of the bone marrow neutrophil progenitors (chapter 2, promyelocytes and myelocytes) separately (Figure 3D). Remarkably, the kinetics of blood neutrophil progenitors are very similar to the bone marrow (aspirate) kinetics, which suggests that blood neutrophil progenitors are released from the bone marrow. However, the percentage of myelocytes within the total neutrophil progenitor population is lower in the bone marrow (chapter 2) compared to the peripheral blood (Figure $3 \mathrm{C}$ ). This might mean that myelocytes are preferably released to the blood compartment from the bone marrow or that these blood neutrophils are not released from the bone marrow, but perhaps from a different compartment like the spleen ${ }^{17}$. Why these neutrophil progenitors are released into circulation remains unclear, but it is possible they are released in order to locally differentiate at the site of inflammation as has been described in eosinophils ${ }^{18}$. Indeed, in mice hematopoietic stem and progenitor cells (HSPCs) survey different organs and they are able to substitute the local production of tissue-resident innate immune cells under homeostatic conditions and in response to inflammation ${ }^{19}$. Alternatively, it is possible that these cells "simply" leak from the bone marrow vasculature into the blood without a distinct function. The latter reason is less likely, because metamyelocytes and banded neutrophils are not found in the peripheral blood during homeostasis (data not shown). 
A
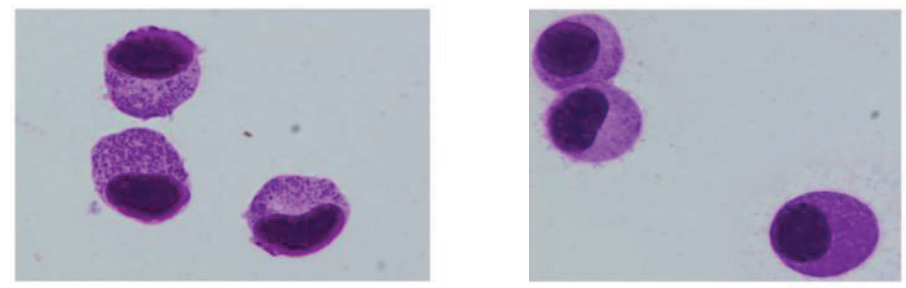

B
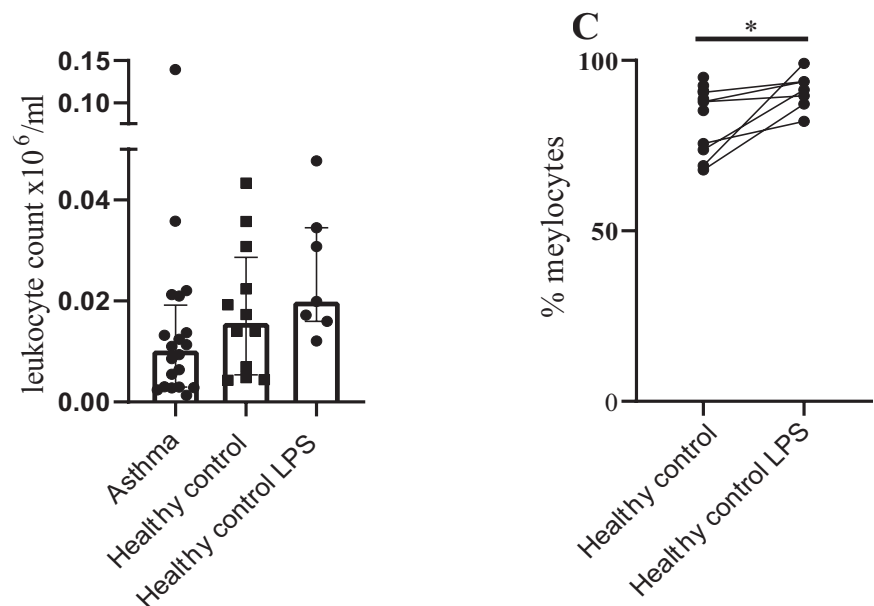

D

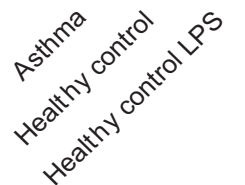

DNA enrichment

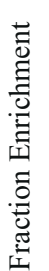

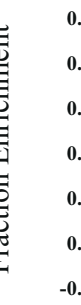

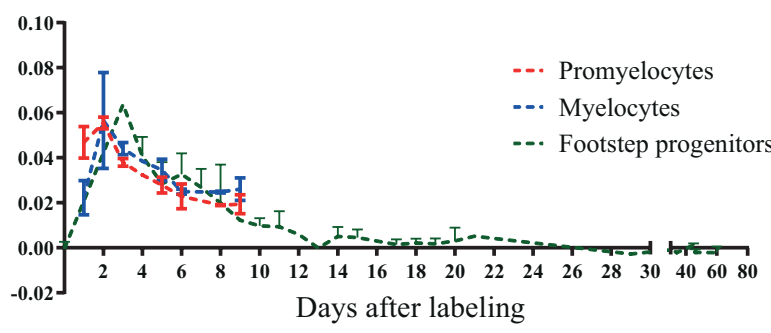

Figure 3. Presence of neutrophil progenitors in the peripheral blood.

SSC high $, C D 193^{\text {neg }}, C D 14^{\text {neg }}, C D 16^{\text {neg }}$ and CD62 $L^{\text {neg }}$ cells were FACS sorted. Cytospins were stained with May-Grunwald-Giemsa and the slides were microscopically evaluated. A. Two representative cytospins of two eosinophilic asthma patients are shown (Objective used: 100x, Axioskop 40, Light microscopy, Carl Zeiss). B. The absolute count of neutrophil progenitors was determined in eosinophilic asthma patients at baseline $(n=20)$, in healthy volunteers $(n=12)$ and after endotoxemia $(n=7)$. C. The percentage of myelocytes (CD11b ${ }^{\text {pos }}$ cells) were determined within the total population of all CD16 ${ }^{\text {neg }}$ and CD62 ${ }^{\text {neg }}$ neutrophils in healthy volunteers $(n=12)$ and in 7 volunteers after endotoxemia $(n=7)$. D. The ratio of ${ }^{2} \mathrm{H}$-labelled and total DNA of neutrophilic promyelocytes (red), myelocytes (blue) in the bone marrow of healthy volunteers and in the blood of eosinophilic asthma patients (green) were determined, similarly as is described in chapter 2 and 4 . 


\section{How can GM-CSF alter CXCR4 functionality in normal granulopoiesis?}

We have shown in chapter 5 that $\mathrm{CXCL} 12$-induced increase in $\left[\mathrm{Ca}^{2+}\right]_{i}$ is completely blocked by stimulation (of neutrophils) with GM-CSF. CXCL12 does not only lead to changes in $\left[\mathrm{Ca}^{2+}\right]_{i}$ but other pathways like phosphorylation of MAP kinases and JAK/ STAT activation are also induced by CXCL12 binding to its receptor ${ }^{20,21}$. It must be emphasized, however, that many of these activation pathways were not established in human neutrophils but only in cell lines or other leukocytes. It is, therefore, possible that many of these pathways are not very relevant in neutrophils. This should be further investigated.

The difficulty with the data obtained with GM-CSF is that most of the above mentioned pathways (with the exception of changes in $\left[\mathrm{Ca}^{2+}\right]_{i}$ ) are also activated by this cytokine ${ }^{22}$. For example, phosphorylation of ERK or P38 in neutrophils continues to be present at least an hour post GM-CSF activation which makes it difficult to determine whether CXCL12 was able to phosphorylate ERK or P38 in GM-CSF treated cells (Figure 4A) as CXCL12 induced ERK phosphorylation is so minor (Chapter 5). Moreover, functional tests to establish whether CXCR4 is inhibited by GM-CSF also proved to be a challenge, because CXCL12 and GM-CSF share a lot of functional characteristics, in which GM-CSF is even (far) more potent, e.g.: priming of fMLF induced ROS production ${ }^{23}$, degranulation/ activation (chapter 5, Supplementary Figure 1 ) and chemokinesis ${ }^{24}$. Chemotaxis could be a good candidate to test for functional differences, but unfortunately, we failed to show any chemotactic response to CXCL12 in our experimental setup (Chapter 5, Supplementary Figure 2). We, however, succeeded in showing an inhibition of CXCL12 induced actin polymerization by GM-CSF in a time dependent manner (Figure 4B). This last finding suggests that the inhibitory effect of GM-CSF might be relevant in vivo as well. So, as explained in Chapter 5, the inhibition of CXCR4 by GM-CSF might result in an acute neutrophil release from the bone marrow to the blood, similarly to what is seen after treatment with plerixafor ${ }^{25}$. Alternatively, GM-CSF might result in an inhibition of CXCR4 induced homing of senescent neutrophils from the blood to the bone marrow ${ }^{26}$. Since, we did not find any suggestion of chemotaxis to CXCR4 (also not after aging neutrophil in vitro for 6 hours), the latter mechanism seems less likely. A study in which treatment with GM-CSF (or plerixafor) is given as a single dose, could elucidate normal CXCR4 functionality in vivo. For example, studying the phenotype of neutrophils in the peripheral blood shortly after such treatment might teach us whether neutrophils have an immature, senescent phenotype or both. 
A

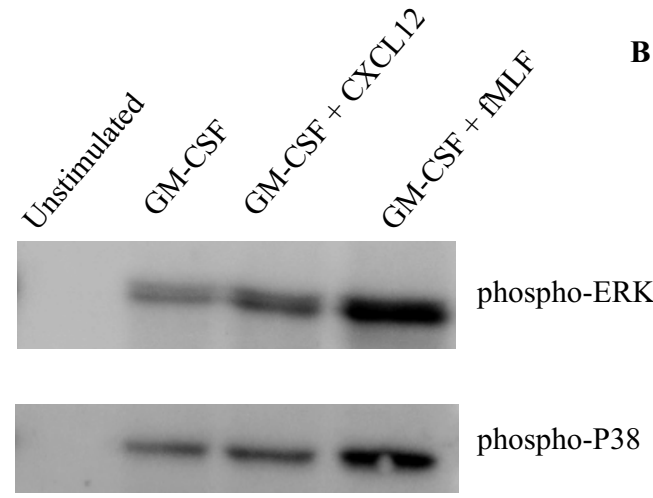

B

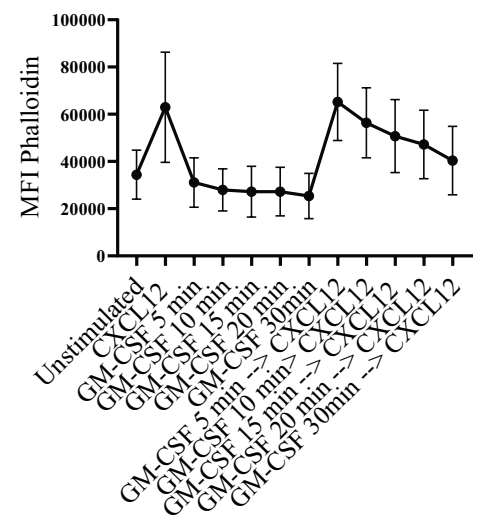

Figure 4. GM-CSF signaling compared to CXCL12 signaling.

A. Two representative western blots of freshly isolated neutrophils that were stained with phospho-ERK and phospho-P38 after stimulation with 100 pM GM-CSF alone or in combination with $100 \mathrm{nM} \mathrm{CXCL12}$ (during 2 min and one hour after GM- CSF) or $10 \mathrm{nM} \mathrm{fMLF} \mathrm{(during} 2$ min and one hour after GM-CSF). B. Neutrophils in isolated leukocytes were gated based on FSC/SSC characteristics and FITC phalloidin signal was measured using flow cytometry. Cells were unstimulated or stimulated with $100 \mathrm{nM}$ CXCL12 during 2 minutes and/or with $100 \mathrm{pM} \mathrm{GM}$-CSF according to the time schedule as is indicated on the X-axis. The median with IQR of three representative experiments is shown.

\section{Are granulocyte kinetics accompanied by slow down-labeling and the existence of multiple peaks?}

In chapter 4 and 9 neutrophil, eosinophil and basophil kinetics are shown in patients with eosinophilic asthma. Especially in eosinophils, days after the first administration of mepolizumab, the loss of ${ }^{2} \mathrm{H}$-enrichment seems to be accompanied by appearance of multiple peaks (Figure 5A). These peaks seem to be four to five days apart. In neutrophils a second peak (three to four days after the first peak) also seems present but less clear (Figure 5B). A suggestion for two peaks, also 3 days apart, is also seen in neutrophil progenitors in the blood of eosinophilic asthma patients (chapter 4 and Figure $5 \mathrm{C}$ ). The obvious explanation for at least neutrophils is the dilution of label caused by multiple cell division of granulocyte progenitors. Alternatively, it is possible that these additional peaks represent the influx of newly labelled granulocytes from the bone marrow from a separate slowly maturing progenitor pool.

In favor of the first hypothesis is the finding that the granulocytes in the second peak are originating from the next division of (the fastest) cells, since label is diluted around two times. This can only be true if the majority of dividing granulocyte progenitors (e.g. promyelocytes) do not enter the post mitotic pool (PMP) after one division. So, a significant number of progenitors need to divide at least twice before entering the maturation program (post-mitotic phase). This implies that the time between two consequent complete cell cycles of neutrophil progenitors takes approximately 3 days 
and for eosinophils 4-5 days. (chapter 2). However, this does not fit with multiple findings in the literature describing complete cell cycle times of neutrophil progenitors between $14 \mathrm{hrs}^{27,28}$ and $20 \mathrm{hrs}^{29}$. Another argument against a cell cycle time of 3 days is the fact that maximum enrichment, especially in blood neutrophils is reached within 1-2 days and not 3 days (chapter 4 ). This finding suggest that neutrophil progenitors enter the post mitotic pool faster than 3 days. These conclusions must be strengthened by appropriate mathematical modelling applying the conveyor belt model described below (and chapter 2 ).

A

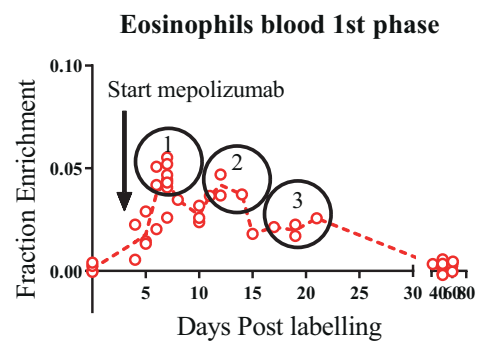

C

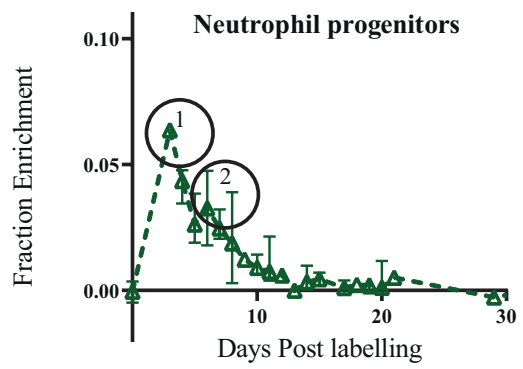

B

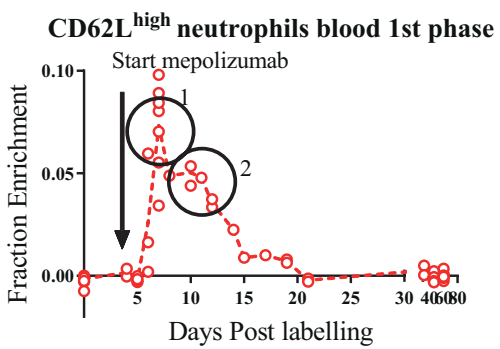

D

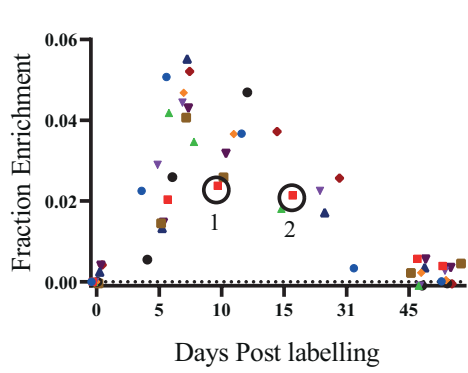

- Patient 1

- Patient 3

4 Patient 5

v Patient 8

- Patient 12

- Patient 13

- Patient 16

- Patient 17

- Patient 18

- Patient 20

Figure 5. Appearance of multiple peaks in granulocyte kinetics

The ratio of ${ }^{2} \mathrm{H}$-labelled and total DNA of blood eosinophils (A), mature neutrophils (B) or neutrophil progenitors (C) was determined before label intake and at several days after label intake for patients with eosinophilic asthma, similarly as is shown in chapter $\mathbf{4}$ and $\mathbf{9}$. For $\mathbf{A}-\mathbf{C}$ the values of multiple patients were combined. Circles indicate the probable presence of multiple peaks. In $\mathbf{D}$, the values of $\mathbf{A}$ are now shown separately per individual. The circles in $\mathbf{D}$ indicate the values of patient 3 that give a suggestion of an existence of a plateau or multiple peaks in eosinophil ${ }^{2} \mathrm{H}$-enrichment.

The second hypothesis assuming an additional slowly maturing progenitor pool is supported by various arguments. First of all, the first hypothesis does not seem to be sustainable (see above). This is strengthened by the finding that the DNA does not dilute label for up to 12 days in eosinophils after treatment with mepolizumab and rather is characterized by a long plateau of 7 days (see Figure 5D). Whether there are multiple peaks is difficult to decipher because of the design of the study only collecting a limiting number of samples. In only one donor (patient 3 ) we see similar ${ }^{2} \mathrm{H}$-enrichment on day 10 and on day 17 after label intake which might fit with the existence of multiple peaks. 
A slow maturing pool has also been suggested by studies showing a so-called lazy pool of progenitors that exhibit long cell cycle times ${ }^{30,31}$. These slow cells can enter the post-mitotic pool at different times causing a slowdown in labeling. It must, however, be emphasized that such slow pool will also be lowly enriched in deuterium during a 6 hours pulse labeling.

\section{How long is the circulatory lifespan of eosinophils?}

In chapter $\mathbf{2}$ we have determined that the circulatory lifespan of neutrophils is at least 2 days. We estimated this lifespan based on the assumption that granulopoiesis takes place according to a conveyor belt model as is explained in chapter $\mathbf{2}^{32}$. Besides, three other assumptions were made to determine the lifespan:

1. The time in which banded neutrophils mature into segmented neutrophils is approximately 2 days.

2. The total segmented neutrophil population is at least as large as the banded neutrophil population (hence a similar maximum ${ }^{2} \mathrm{H}$-enrichment in both cell populations in the bone marrow).

3. During homeostasis all banded neutrophils feed similarly into each segmented neutrophil population, regardless of the compartment (bone marrow, blood or oral rinse).

For eosinophils we do not have ${ }^{2} \mathrm{H}$-enrichment data of their progenitors in the bone marrow. However, sufficient information is available in order to make a similar prediction for the eosinophil lifespan. Firstly, the post mitotic pool transit time for eosinophils is shorter compared to neutrophils: approximately 4 days in eosinophils versus 6 days in neutrophils (compare chapter 2 and 4 with chapter 9). This difference might be explained by the fact that eosinophils do not have a banded stage (chapter 7). This would mean that the metamyelocyte stage in eosinophils is the last stage before the full maturation of eosinophils. The time between both stages might also be approximately 2 days, since this would account for the 2 days shorter PMPtt for eosinophils compared to neutrophils. However, it should be emphasized that it is not likely that mature eosinophils from the bone marrow have the same kinetics compared to blood eosinophils, because of their phenotypical dissimilarity (lower CD193, IL$3 R \alpha$ and IL-5R $\alpha$ expression in bone marrow eosinophils compared to blood, chapter 7). Therefore, it is plausible that bone marrow eosinophils are still on the conveyor belt and those cells might stay on the conveyor for at least one day. Now, because the blood eosinophil compartment is approximately as large as the blood eosinophil compartment (chapter 7) and with the same reasoning as with neutrophils, the circulatory lifespan of eosinophils might be at least one day. This is in line with an earlier study in which eosinophil lifespan in the circulation was determined to be 25 hours $^{33}$. However, because ${ }^{2} \mathrm{H}$-label is diluted in sputum eosinophils (chapter 9), the actual lifespan of eosinophils (in the tissues) is undoubtedly longer.

To test whether above described prediction is correct, actual bone marrow eosinophil labelling data should be collected. 


\section{Why are peripheral blood eosinophil kinetics back to normal after long-term treatment with mepolizumab?}

In chapter 9, we showed that after treatment with mepolizumab, it only takes two days for the amount of peripheral blood eosinophils to be strongly reduced. The reduction in eosinophils is persistent beyond at least 80 days (Figure 2A of chapter 9). As was explained in chapter $\mathbf{9}$, short term treatment with mepolizumab led to delay in down labelling of ${ }^{2} \mathrm{H}$-enrichment. Surprisingly, this effect is completely lost during the second labelling phase, which means that long-term treatment with mepolizumab caused an adaptation of kinetics, regardless of the number of eosinophils. It is difficult to understand the mechanism behind this adaptation as long as we are not completely certain why eosinophil kinetics is delayed in the first place. There are, however, two concepts that might provide a possible explanation.

Concept 1: 4 days of treatment leads to an intermediate unbalanced situation. It is important to realize that the start of treatment with mepolizumab was already four days after the intake of the deuteriated glucose pulse, which means that during the first 4 days after the label intake, the amount of label incorporation in DNA of eosinophil progenitors (e.g. eosinophilic promyelocytes) is equal in both placebo and verum group. These eosinophil progenitors with label incorporated in their DNA are on the "conveyor belt" (see chapter 2). Then, when mepolizumab was started four days after labelling, this first wave of eosinophil progenitors was well on their way on the conveyor belt (postmitosis). In the placebo group such conveyor belt was "loaded" by the same absolute amount of (un)labeled eosinophil progenitors which consequently diluted the ${ }^{2} \mathrm{H}$-enriched DNA in total eosinophil population to the same extend. This led to a situation with similar peak enrichments at the same time in both groups. After the start of mepolizumab, the absolute number of eosinophilic promyelocytes and myelocytes decreased in the bone marrow, because IL-5 is growth factor rather than a differentiation factor for eosinophils ${ }^{34,35}$. So in the mepolizumab group, the dilution caused by the influx of new unlabeled eosinophil progenitors on the conveyor belt is less pronounced. Therefore, the relative amount of label remains high compared to the placebo group, whereas the absolute number of cells lower. After the new labelling experiment, this conception is not relevant anymore, because under these balanced conditions only the number of eosinophils is affected as only proliferation is affected and not differentiation.

Concept 2: redundant cytokines. Another explanation is that kinetics of the remaining eosinophils after mepolizumab have adapted as a result of the presence of different cytokines that might take over the effect of IL-5. Both IL-3 and GM-CSF are likely candidates, since they share the common ß-chain with IL- $5^{36}$. Indeed IL-3R $\alpha$-expression on peripheral blood eosinophils decreased after treatment with mepolizumab, possibly caused by IL-3 induced downregulation. Besides, both IL-3R $\alpha$ and GM-CSFR $\alpha$ are high in BAL eosinophils after a segmental allergen challenge, whereas IL-5R $\alpha$ expression is low, demonstrating the redundancy of IL-5 for (tissue) eosinophils ${ }^{13}$.

It should, however be emphasized that the concentrations of IL-3, IL-5 and GM-CSF in the blood and sputum remained below the detection limit after treatment with mepolizumab (data not shown). Still, it is possible that the relevant concentrations of 
these cytokines are only reached in the bone marrow, where eosinophilopoiesis manly takes place ${ }^{37}$. In addition, the concept is not supported by the fact that ß-common/ ß-IL-3 double knock out mice (that also lack the IL-3R and GM-CSFR) also have normal eosinophils, albeit at low levels ${ }^{38}$. Again, it is likely, as was also explained in chapter 9 that signaling through the beta-common receptor is not essential for eosinophil differentiation, but rather important for proliferation (the number of eosinophil progenitors that divide).

Why are sputum eosinophils hardly ${ }^{2} \mathrm{H}$ enriched after treatment with mepolizumab? In chapter 4 (Figure 4B), we have shown that neutrophils in the sputum have similar kinetics compared to blood neutrophils. This implies that sputum and blood neutrophils are most likely in direct and complete exchange with each other. Therefore, sputum neutrophils are probably not longer lived in the sputum compared to the blood. This is in contrast to the kinetics of blood and sputum eosinophils, which are not directly comparable (chapter 9). Especially after treatment with mepolizumab (Figure 5 in chapter 9), sputum eosinophils lose their ${ }^{2} \mathrm{H}$-label rapidly (e.g. 14 days after the second labelling period sputum eosinophils $(n=4)$ did not have any ${ }^{2} \mathrm{H}$-label, whereas ${ }^{2} \mathrm{H}$-enrichment in the DNA of blood eosinophils is around $3 \%$ ). This remarkable difference is unexpected and difficult to interpret, because information about ${ }^{2} \mathrm{H}$-enrichment between day 8 and 13 after label intake is missing in our experimental setup. Still, several speculations can be made:

1. ${ }^{2} \mathrm{H}$-label in the sputum might be lost because eosinophils in the sputum, coming from the blood are rapidly dying. This is not completely unimaginable, since IL-5 is a known survival factor for eosinophils ${ }^{39,40}$. However, this occurrence cannot be the only explanation as it should have been accompanied by a higher maximum ${ }^{2} \mathrm{H}$-enrichment for sputum eosinophils after mepolizumab as a result of less dilution by the presence of longer-lived unlabeled eosinophils. Still, it is possible that this higher eosinophil ${ }^{2} \mathrm{H}$-enrichment is missed, because the peak is somewhere between day 8 and 13 after label intake.

2. Eeosinophils might hardly home to the sputum after treatment with mepolizumab. IL-5 is known to prime eosinophils for activation and transmigration to the airways ${ }^{41,42}$. Therefore, sputum cells were not labeled because blood labelled eosinophils never reached this compartment. Also, this explanation cannot fully account for this finding, since the initial eosinophil ${ }^{2} \mathrm{H}$-enrichment was as high and as early compared to placebo.

3. Finally, sputum eosinophils might not come from blood eosinophils. So, the kinetics found in the sputum were not a reflection of blood eosinophil kinetics. Although, this is not a very likely explanation, multiple studies have shown that local eosinophilopoiesis (proliferation of early eosinophil progenitors in situ) is important in severe asthmatics ${ }^{18,43}$. So, targeting IL-5, could have resulted in the inhibition of local eosinophilopoiesis, as well, leading to less ${ }^{2} \mathrm{H}$-label incorporation in the sputum. 


\section{References}

1. Gallin, J. I. Human neutrophil heterogeneity exists, but is it meaningful? Blood $63,977-83$ (1984).

2. Ng, L. G., Ostuni, R. \& Hidalgo, A. Heterogeneity of neutrophils. Nat. Rev. Immunol. 19, 255-265 (2019).

3. Pillay, J. et al. A subset of neutrophils in human systemic inflammation inhibits $T$ cell responses through Mac-1. J. Clin. Invest. 122, 327-36 (2012).

4. Tak, T. et al. Human CD62Ldim neutrophils identified as a separate subset by proteome profiling and in vivo pulse-chase labeling. Blood 129, 3476-3485 (2017).

5. Leliefeld, P. H. C. et al. Differential antibacterial control by neutrophil subsets. Blood Adv. 2, 1344-1355 (2018).

6. Scapini, P., Marini, O., Tecchio, C. \& Cassatella, M. A. Human neutrophils in the saga of cellular heterogeneity: insights and open questions. Immunol. Rev. 273, 48-60 (2016).

7. Brandau, S. et al. Myeloid-derived suppressor cells in the peripheral blood of cancer patients contain a subset of immature neutrophils with impaired migratory properties. J. Leukoc. Biol. 89, 311-317 (2011).

8. Carmona-Rivera, C. \& Kaplan, M. J. Low-density granulocytes: a distinct class of neutrophils in systemic autoimmunity. Semin. Immunopathol. 35, 455-463 (2013).

9. Kamp, V. M. et al. Modulation of granulocyte kinetics by GM-CSF/IFN- $\gamma$ in a human LPS rechallenge model. J. Leukoc. Biol. 94, 513-20 (2013).

10. Casanova-Acebes, M. et al. Rhythmic modulation of the hematopoietic niche through neutrophil clearance. Cell 153, 1025-35 (2013).

11. Ella, K., Csépányi-Kömi, R. \& Káldi, K. Circadian regulation of human peripheral neutrophils. Brain. Behav. Immun. 57, 209-221 (2016).

12. Mesnil, C. et al. Lung-resident eosinophils represent a distinct regulatory eosinophil subset. J. Clin. Invest 126, 3279-3295 (2016).

13. Kelly, E. A. et al. Mepolizumab Attenuates Airway Eosinophil Numbers, but Not Their Functional Phenotype in Asthma. Am. J. Respir. Crit Care Med (2017) doi:10.1164/ rccm.201611-22340C [doi].

14. U, Hollenstein, M, Homoncik PJ, Stohlawetz, C, Marsik, A, S. \& HG, Eichler, B, J. Endotoxin Down-Modulates Granulocyte Colony-Stimulating Factor Receptor (CD114) on Human Neutrophils. J. Infect. Dis. 182, (2000).

15. Manz, M. G. \& Boettcher, S. Emergency granulopoiesis. Nat. Rev. Immunol. 14, 302-314 (2014).

16. L.Schudel. Leitfaden der Blutmorphologie: Manual of blood morphology. (1965).

17. Jhunjhunwala, S. et al. Frontline Science: Splenic progenitors aid in maintaining high neutrophil numbers at sites of sterile chronic inflammation. J. Leukoc. Biol. 100, 253-60 (2016).

18. Sehmi, R. et al. Role of local eosinophilopoietic processes in the development of airway eosinophilia in prednisone-dependent severe asthma. Clin. Exp. Allergy 46, 793-802 (2016).

19. Massberg, S. et al. Immunosurveillance by hematopoietic progenitor cells trafficking through blood, lymph, and peripheral tissues. Cell 131, 994-1008 (2007).

20. Busillo, J. M. \& Benovic, J. L. Regulation of CXCR4 signaling. Biochim. Biophys. Acta 1768, 952-63 (2007). 
21. Pawig, L., Klasen, C., Weber, C., Bernhagen, J. \& Noels, H. Diversity and Inter-Connections in the CXCR4 Chemokine Receptor/Ligand Family: Molecular Perspectives. Front. Immunol. 6, 429 (2015).

22. Hercus, T. R. et al. The granulocyte-macrophage colony-stimulating factor receptor: linking its structure to cell signaling and its role in disease. Blood 114, 1289-98 (2009).

23. Kitagawa, S. et al. Recombinant human granulocyte colony-stimulating factor enhances superoxide release in human granulocytes stimulated by the chemotactic peptide. Biochem. Biophys. Res. Commun. 144, 1143-6 (1987).

24. N, H. et al. Random Migration of Polymorphonuclear Leukocytes Induced by GM-CSF Involving a Signal Transduction Pathway Different From That of fMLP. J. Leukoc. Biol. 61, (1997).

25. Hendrix, C. W. et al. Pharmacokinetics and safety of AMD-3100, a novel antagonist of the CXCR-4 chemokine receptor, in human volunteers. Antimicrob. Agents Chemother. 44, 1667-73 (2000).

26. Suratt, B. T. et al. Role of the CXCR4/SDF-1 chemokine axis in circulating neutrophil homeostasis. Blood 104, 565-71 (2004).

27. Stryckmans, P., Cronkite, E. P., Fache, J., Fliedner, T. M. \& Ramos, J. Deoxyribonucleic acid synthesis time of erythropoietic and granulopoietic cells in human beings. Nature 211, 717-20 (1966).

28. Constable, T. B. \& Blackett, N. M. The cell population kinetics of neutrophilic cells. Cell Tissue Kinet. 5, 289-302 (1972).

29. Knowes, D. M. Neoplastic hematopathology. (2001).

30. LAJTHA, L. G., GILBERT, C. W., PORTEOUS, D. D. \& ALEXANIAN, R. KINETICS OF A BONEMARROW STEM-CELL POPULATION. Ann. N. Y. Acad. Sci. 113, 742-52 (1964).

31. Dresch, C., Troccoli, G. \& Mary, J. Y. Growth fraction of myelocytes in normal human granulopoiesis. Cell Tissue Kinet. 19, 11-22 (1986).

32. Cartwright, G. E., Athens, J. W. \& Wintrobe, M. M. The kinetics of granulopoiesis in normal man. Blood 24, 780-803 (1964).

33. Farahi, N. et al. Use of 111-Indium-labeled autologous eosinophils to establish the in vivo kinetics of human eosinophils in healthy subjects. Blood 120, 4068-4071 (2012).

34. Menzies-Gow, A. et al. Anti-IL-5 (mepolizumab) therapy induces bone marrow eosinophil maturational arrest and decreases eosinophil progenitors in the bronchial mucosa of atopic asthmatics. J. Allergy Clin. Immunol 111, 714-719 (2003).

35. Sanderson, C. J. Interleukin-5, eosinophils, and disease. Blood 79, 3101-3109 (1992).

36. Martinez-Moczygemba, M. \& Huston, D. P. Biology of common beta receptor-signaling cytokines: IL-3, IL-5, and GM-CSF. J. Allergy Clin. Immunol 112, 653-665 (2003).

37. Rohde, D. et al. Cytokine release by human bone marrow cells: analysis at the single cell level. Virchows Arch. 424, 389-95 (1994).

38. Nishinakamura, R. et al. Mice deficient for the IL-3/GM-CSF/IL-5 beta c receptor exhibit lung pathology and impaired immune response, while beta IL3 receptor-deficient mice are normal. Immunity 2, 211-22 (1995).

39. Yamaguchi, Y. et al. Analysis of the survival of mature human eosinophils: interleukin-5 prevents apoptosis in mature human eosinophils. Blood 78, 2542-2547 (1991).

40. Owen Jr., W. F. et al. Regulation of human eosinophil viability, density, and function by granulocyte/macrophage colony-stimulating factor in the presence of 3T3 fibroblasts. J. Exp. Med 166, 129-141 (1987). 
41. Johansson, M. W. et al. Anti-IL-5 attenuates activation and surface density of beta(2) -integrins on circulating eosinophils after segmental antigen challenge. Clin. Exp. Allergy 43, 292-303 (2013).

42. Stein, M. L. et al. Anti-IL-5 (mepolizumab) therapy reduces eosinophil activation ex vivo and increases IL-5 and IL-5 receptor levels. J. Allergy Clin. Immunol 121, 1473-1483,1483 (2008).

43. Wood, L. J., Inman, M. D., Denburg, J. A. \& O’Byrne, P. M. Allergen Challenge Increases Cell Traffic between Bone Marrow and Lung. Am. J. Respir. Cell Mol. Biol. 18, 759-767 (1998). 


\section{Appendix}

Nederlandse samenvatting

Dankwoord

Curriculum Vitae 


\section{Nederlandse samenvatting}

\section{Inleiding}

Het immuunsysteem bestaat uit meerdere cellen en moleculen die tezamen de functie hebben om zowel externe als interne indringers te bestrijden. De cellen in dit systeem worden de witte bloedcellen (of leukocyten) genoemd en hebben ieder een specifieke functie. Eén van de belangrijkste cellen is de neutrofiele granulocyt. Deze cel is uitstekend in staat om bacteriën (of andere micro-organismen) te herkennen en "op te eten" (fagocyteren). Eenmaal opgegeten heeft de neutrofiel multipele enzymen en allerlei andere moleculen tot zijn beschikking om de bacterie te vernietigen. Als de neutrofiel niet in staat is om micro-organismen op te eten, kan hij als alternatief de toxische inhoud van deze granules (korrels) gecontroleerd naar buitenaf "vrijgeven". Dit proces wordt "degranulatie" genoemd en onderstreept waarom neutrofielen potentieel gevaarlijk kunnen zijn. Uiteraard is de functie van levensbelang omdat de mens zonder deze cellen snel zou bezwijken aan allerlei infecties, maar overactivatie kan juist weer leiden tot onnodig (collaterale) schade zoals bijvoorbeeld wordt gezien in het geval van ARDS (een levensgevaarlijk longsyndroom). Derhalve is het essentieel dat ons lichaam in staat is om de productie, de verspreiding en de opruiming van neutrofielen zo goed mogelijk te reguleren.

De andere cellen die veelvuldig in dit proefschrift worden genoemd, zijn de eosinofiele granulocyten. Deze cellen bezitten net als de neutrofielen meerdere granules, maar de inhoud van deze granules verschilt met die van de neutrofielen. In tegenstelling tot neutrofielen, is de rol van eosinofielen minder duidelijk. In de klassieke leerboeken worden zij omschreven als cellen die gespecialiseerd zijn in de bestrijding van multicellulaire organismen, zoals parasieten. Echter, deze rol is alleen goed beschreven voor de bestrijding van de zogenaamde helminthen (parasitaire wormen). Zij doen dit door tezamen de veel grotere parasiet aan te vallen en te doden. In het geval van andere parasieten, is de rol van de eosinofiel minder duidelijk. In sommige gevallen lijken eosinofielen voor bepaalde parasieten zelfs een symbiotische functie te hebben. Aan de andere kant is het wel duidelijk dat eosinofielen betrokken zijn bij meerdere (allergische) aandoeningen. In het geval van (eosinofiel) astma worden deze cellen door allerlei factoren naar de luchtwegen gerekruteerd en geactiveerd met als gevolg dat ze schade aan de luchtwegen veroorzaken. Dit leidt tot de bekende symptomen van astma: benauwdheid, kortademigheid bij inspanning, hoesten en piepende ademhaling. Het is daarom niet verwonderlijk dat er inmiddels meerdere medicijnen zijn ontwikkeld die specifiek gericht zijn tegen de eosinofiel. Het medicijn wat in hoofdstuk 9 van dit proefschrift wordt gebruikt is mepolizumab. Mepolizumab is een monoklonaal antilichaam gericht tegen IL-5, een cytokine wat essentieel is voor de productie, activatie en overleving van eosinofielen.

\section{Levensloop van granulocyten}

Dit proefschrift richt zich voornamelijk op het beschrijven van de levensloop (kinetiek) van neutrofiele, eosinofiele en in mindere mate de basofiele granulocyten. De levensloop van granulocyten wordt bepaald door: i: aanmaak van de voorlopers in 
het beenmerg, ii: de rijpingstijd van de cellen in het beenmerg, iii: de levensduur van cellen in het bloed en iv: de levensduur van de cellen in het weefsel. Het is belangrijk om meer kennis te vergaren over de levensloop van deze witte bloedcellen, omdat zonder deze kennis bepaalde ziekteprocessen moeilijk te bevatten zijn. Bijvoorbeeld: hoewel mepolizumab leidt tot een lager aantal eosinofiele granulocyten in het bloed van astmapatiënten, is het aantal eosinfielen in de luchtwegen minder aangedaan. Een goede verklaring hiervoor bedenken zonder enige kennis over de levensloop van deze cellen is onmogelijk.

Uiteraard, hebben meerdere studies in het verleden reeds geprobeerd de levensduur van zowel neutrofielen als eosinofielen te bepalen. In deze studies werden echter zeer uiteenlopende conclusies getrokken. De levensduur van een neutrofiel verschilt bijvoorbeeld van enkele uren tot enkele dagen. Dit komt omdat in het verleden er verschillende, maar waarschijnlijk ook verkeerde methodes zijn gebruikt, waardoor de echte levensduur nog niet duidelijk is. Het labelen van granulocyten buiten het lichaam om, kan ertoe leiden dat deze cellen geactiveerd raken en om die reden eerder het bloed verlaten dan zij gewoonlijk zouden doen. Met als gevolg dat er onterecht wordt geconcludeerd dat neutrofielen een korte halfwaardetijd hebben in het bloed.

Gelukkig is er een nieuwe methode bedacht om granulocyten in het lichaam zelf (in vivo) te labelen. Deze methode, genaamd in vivo deuterium labelen, is niet toxisch voor de cellen en veelvuldig gebruikt in dit proefschrift. Deuterium is een waterstofatoom met een extra neutron. Dit maakt dat de deuteriumatomen zwaarder zijn dan normale waterstofatomen zonder dat deze atomen de radioactieve eigenschap hebben. Met andere woorden: deuterium is een stabiele isotoop van waterstof.

In dit proefschrift kregen onze proefpersonen suiker te drinken waarvan twee waterstofatomen zijn vervangen door twee deuteriumatomen. Vervolgens, werd deze gemodificeerde suiker in onze proefpersonen net als normale suiker gebruikt als bouwsteen in het DNA van alle delende cellen. Daarna waren wij in staat om op verschillende dagen de hoeveelheid deuterium in de cellen van interesse in het bloed, het beenmerg of in het slijm te bepalen. Deze informatie gaf ons een idee over hoe snel cellen worden aangemaakt en opgeruimd.

\section{Dit proefschrift}

Dit proefschrift is opgedeeld in 2 delen. Het eerste deel gaat over de levensloop en activatiestatus van neutrofiele granulocyten bij gezonde mensen en mensen met astma. Het tweede deel beschrijft de levensloop en activatiestatus van eosinofiele granulocyten bij gezonde mensen, mensen met eosinofiel astma en mensen met atopische dermatitis (constitutioneel eczeem).

\section{Deel 1. Levensloop en activatiestatus van neutrofiele granulocyten.}

In hoofdstuk 2 van dit proefschrift hebben wij met de hulp van deuterium labelen, getracht de levensduur van neutrofiele granulocyten te bepalen. Deze data is uniek, omdat er voor het eerst deuterium label is bepaald in voorlopers van neutrofielen in het beenmerg van gezonde vrijwilligers. Bovendien, wordt deze data, in tegenstelling 
tot eerdere studies, beschreven volgens de zogenaamde "conveyor belt model" (loopband model). Dit model gaat ervan uit dat de cel die het eerst het bloed bereikt, ook het eerst het bloed weer verlaat. Oudere studies gingen ervan uit -naar ons idee onterecht- dat dit proces stochastisch is. Met de conveyor belt model komen wij tot de conclusie dat neutrofielen granulocyten in het bloed, maar ook in het weefsel een totale levensduur hebben van ten minste 2 dagen.

Hoofdstuk 3 geeft een overzicht van de oorsprong van neutrofielen met een lage(re) dichtheid. Normaal gesproken hebben neutrofielen uit het bloed een hoge dichtheid, maar in het geval van ontstekingsziekten hebben sommige neutrofielen een lagere dichtheid. Deze neutrofielen met een lage densiteit kunnen door middel van FicollPaque (synthetische polymeer) worden gescheiden van andere neutrofielen met een hoge (normale) densiteit. Wij hebben met behulp van de beschikbare literatuur, aangevuld met onze data, geconcludeerd dat neutrofielen altijd (ook in homeostase) verschillende dichtheden hebben. Dit spectrum aan verschillende dichtheden is meest waarschijnlijk gekoppeld aan de leeftijd/ rijpingsgraad van neutrofielen waarin de jongste neutrofielen de laagste dichtheid hebben, terwijl de oudste neutrofielen de hoogste dichtheid hebben. Tijdens systemische ontsteking, zoals het geval is in bepaalde ziektes, worden neutrofielen geactiveerd wat er toe leidt dat de dichtheid van alle neutrofielen verlaagd wordt. Alleen de neutrofielen die in homeostase laag in het spectrum zitten, zullen de drempel bereiken van de lage dichtheid van Ficoll-Paque. Opmerkelijk is dat de neutrofielen met de laagste dichtheid, ook in homeostase, andere unieke karakteristieken hebben. Zij zijn bijvoorbeeld in staat om de proliferatie (deling) van T-cellen te remmen. Ook zijn ze beter in staat om bacteriën te doden in vergelijking met de neutrofielen met de hoogste dichtheid.

In hoofdstuk 4 wordt opnieuw deuterium glucose gebruikt, maar deze keer om patiënten met eosinofiel astma te labelen. In totaal hebben wij 20 patiënten geïncludeerd waarvan de helft blind werd gerandomiseerd voor behandelingen met mepolizumab en de andere helft kreeg placebo. Vervolgens hebben we in dit hoofdstuk gekeken naar de levensloop van neutrofiele granulocyten in het bloed en sputum. Wij hadden namelijk de hypothese dat neutrofiele granulocyten naar de longen worden gerekruteerd als response op inflammatie veroorzaakt door eosinofielen. Als anti-IL-5 ertoe leidt dat er minder eosinofiele granulocyten zijn in de luchtwegen van astmatici, dan zou dat wellicht ook leiden tot minder aanwezigheid van neutrofielen in dezelfde luchtwegen. Dit bleek echter niet zo te zijn. We zagen namelijk geen enkel verschil tussen de twee groepen als het gaat om aantal, aanmaak of levensduur van neutrofielen in beide compartimenten. We hebben daarnaast door middel van deuterium glucose bevestigd dat er drie "groepen" neutrofielen in het bloed van astmatici aanwezig zijn: neutrofiele voorlopers (snel gelabeld), normale gesegmenteerde neutrofielen (normaal gelabeld) en hypergesegmenteerde neutrofielen (langzaam gelabeld). Daarnaast hebben we bevestigd dat neutrofiele granulocyten in het sputum geen andere levensloop hebben vergeleken met neutrofielen in het bloed. Dat wil zeggen: of beide compartimenten hebben een directe uitwisseling met elkaar, of neutrofielen in het sputum gaan snel dood op het moment dat zij de longen bereiken. 
In hoofdstuk 5 hebben wij de rol van CXCR4 in vers uit het bloed geïsoleerde neutrofielen onderzocht. CXCR4 is een chemokine receptor waarvan de ligand CXCL12 is. In de literatuur bestaat de consensus dat in het geval van gesegmenteerd neutrofielen (rijpe neutrofielen) deze receptor alleen een rol speelt als de cellen (buiten het lichaam) verouderd zijn of zich in het beenmerg bevinden. Wij hebben echter laten zien dat CXCL12 ook in verse neutrofielen uit het bloed de cellen activeert: er onstaat namelijk een stijging van $\left[\mathrm{Ca}^{2+}\right]$, lichte "degranulatie" van neutrofielen en "vooractivatie" van de oxidatieve burst. Bovendien zagen wij dat GM-CSF (een bekende cytokine die neutrofielen activeert) in staat is om snel CXCR4 in neutrofielen te remmen. Deze bevinding zou van klinisch belang kunnen zijn, omdat CXCR4 een belangrijke rol speelt in het "behouden" van neutrofielen in het beenmerg. Remming van CXCR4 kan dus ertoe leiden dat deze cellen worden vrijgegeven in het bloed. Dit zou nader onderzocht moeten worden.

\section{Deel 2. Levensloop en activatiestatus van eosinofiele granulocyten.}

Eosinofiele granulocyten zijn wat betreft aanmaak, activatie en overleving grotendeels afhankelijk van IL-5. Deze cytokine is daarnaast vrijwel volledig eosinofiel specifiek. Alleen bij basofiele granulocyten is ook onomstotelijk bewezen dat zij de IL-5 receptor bezitten. IL-5 gerichte therapieën zullen dus met name effect hebben op eosinofielen en in mindere mate basofielen. Dit is in hoofdstuk 6 samengevat door een overzicht te geven wat er in de literatuur bekend is. Eén van de belangrijkste bevindingen uit de literatuur is dat naast mepolizumab ook reslizumab en benralizumab gericht zijn tegen IL-5. Reslizumab is net als mepolizumab een mononklonaal antilichaam dat bindt aan IL-5 zelf, waardoor deze cytokine niet meer in staat is om te binden aan de IL-5 receptor. Benralizumab werkt anders: dit monoklonale antilichaam bindt aan de IL-5 receptor zelf. Hierdoor wordt de immuuncel waaraan het bindt, gelabeld als zijnde een vreemd voorwerp. Het immuunsysteem reageert hierop en ruimt deze cellen op: antilichaam afhankelijke cellulaire cytotoxiciteit (ADCC). Dus, mepolizumab en reslizumab zorgen ervoor dat er minder eosinofielen worden aangemaakt, terwijl benralizumab leidt tot een totale depletie van eosinofielen. Vanwege ADCC worden ook basofielen volledig gedepleteerd.

In hoofdstuk 7 hebben wij voor het eerst het fenotype van eosinofiele voorlopers gekarakteriseerd. Dit hebben we gedaan door gebruik te maken van twee markers op het celoppervlak: $\mathrm{CD} 11 \mathrm{~b}$ en $\mathrm{CD} 62 \mathrm{~L}$. Met de combinatie van deze twee markers konden we 3 eosinofiele voorlopers identificeren: promyelocyten (CD $11 b^{\text {neg }} C D 62 L^{\text {neg }}$ ), myelocyten (CD11 $\left.{ }^{\text {pos }} \mathrm{CD} 62 \mathrm{~L}^{\text {neg }}\right)$, metamyelocyten $\left(\mathrm{CD} 11 \mathrm{~b}^{\text {pos }} \mathrm{CD} 62 \mathrm{~L}^{\mathrm{dim}}\right)$ en rijpe eosinofielen ( $C D 11 b^{\text {pos }} C D 62 L^{\text {pos }}$ ). Dit is een belangrijke ontdekking, omdat vanaf nu op vrij eenvoudige wijze eosinofiele voorlopers in het beenmerg herkend en gefenotypeerd kunnen worden, ook in ziekte.

Deze voorlopers werden vooralsnog alleen geïdentificeerd op basis van morfologie. Aanvullende experimenten zijn nodig, zoals het in kaart brengen van het mRNA en/of het uitvoeren van in vitro celkweken, om onomstotelijk bewijs te vergaren dat het hier inderdaad gaat om de eosinofiele voorlopers.

In hoofdstuk 8 hebben we direct de vergaarde kennis over eosinofiele voorlopers in het beenmerg gebruikt om te onderzoeken wat er gebeurt tijdens humane endotoxemie. 
Humane endotoxemie is een goed model voor acute inflammatie. In dit model krijgen proefpersonen een lage concentratie LPS ingespoten (bacterieel product) wat ertoe leidt dat het aangeboren immuunsysteem (massaal) wordt geactiveerd. Net zoals tijdens sepsis, leidt LPS toediening tot een evidente eosinopenie. Aangezien wij in het beenmerg geen verschil zagen in het aantal eosinofiele voorlopers, hebben we kunnen concluderen dat deze eosinopenie (alleen) wordt veroorzaakt door verdwijning van eosinofielen uit het bloed. Waarschijnlijk gaan de eosinofielen massaal naar het weefsel. Echter deze hypothese moet nog nader worden onderzocht.

In hoofdstuk 9 hebben we dezelfde twintig proefpersonen met eosinofiel astma gebruikt, als in hoofdstuk 4, maar in dit hoofdstuk hebben we gekeken naar de levensloop van eosinofiele en basofiele granulocyten in het bloed en sputum. Uit deze studie hebben we bevestigd dat behandeling met mepolizumab snel (na twee dagen al) leidt tot een vermindering van eosinofiele granulocyten in het bloed, terwijl er geen verschil was na behandelingen met placebo. Daarnaast was het DNA uit bloed eosinofielen langer verrijkt met deuterium, gedurende de eerste behandeling met mepolizumab, vergeleken met placebo. Deze bevinding kan worden veroorzaakt door: i verminderde homing van eosinofielen naar de luchtwegen of ii vertraagde productie van eosinofielen in het beenmerg of iii beiden. Nadat we voor de tweede keer hadden gelabeld met deuterium glucose, vonden we opmerkelijk genoeg, geen verschil meer in deuterium verrijking. Dit is zeer waarschijnlijk bewijs voor het feit dat de normale aanmaak van eosinofielen, net als in de muis, niet afhankelijk is van IL-5. Het omhoogdrijven van de productie van eosinofielen is daarentegen wel afhankelijk van IL-5.

Verder hebben we bevestigd dat het aantal eosinofielen in het sputum na ten minste 42 dagen afneemt in vergelijking met placebo. Dit is zeer waarschijnlijk het gevolg van een combinatie van verminderde homing van eosinofielen vanuit het bloed naar de luchtwegen en versnelde celdood.

In tegenstelling tot eosinofielen, heeft mepolizumab geen effect op het aantal basofiele granulocyten of hun levensloop in het bloed.

Ten slotte, hebben we in hoofdstuk 10 gekeken naar de effecten van dupilumab op eosinofiele granulocyten in constitutioneel eczeem. Dupilumab is een monoklonaal antilichaam, gericht tegen de alpha-keten van IL-4 receptor. Dit deel van de receptor zit ook vast aan de IL-13 receptor. Dupilumab remt dus zowel de effecten van IL-4 als IL13. Beiden cytokines zijn essentieel in de pathogenese van constitutioneel eczeem. Dit medicijn werkt daarom goed in het verbeteren van symptomen in dit ziektebeeld. Het medicijn heeft echter ook een mogelijk ongewenst effect tot gevolg: het zorgt voor een (tijdelijke) toename van eosinofielen in het bloed. Wij hebben bevestigd dat dit zeer waarschijnlijk wordt veroorzaakt door verminderde migratie van eosinofielen naar de huid. Dit effect wordt teweeggebracht door verminderde productie van eotaxines in de weefsel(s). Daar, wij in dit hoofdstuk hebben laten zien dat eosinofiele granulocyten in CE verhoogde activatie hebben, is dit effect (op langere termijn) potentieel gevaarlijk. Het is daarom raadzaam om het aantal eosinofielen in het bloed te monitoren tijdens behandeling met dupilumab, of in ieder geval in het begin. 


\section{Conclusie}

In dit proefschrift hebben wij grote stappen genomen om de levensloop van granulocyten beter in kaart te brengen. Zo hebben we vastgesteld dat neutrofiele granulocyten een levensduur hebben van minimaal 2 dagen. Deze levensduur geldt voor ieder compartiment: bloed, beenmerg of weefsel. Verder hebben we bevestigd dat inflammatie ertoe kan leiden dat de samenstelling en activatie van neutrofielen in het bloed verandert. Beiden hebben effect op de algehele dichtheid van deze cellen.

Verder hebben we laten zien dat mepolizumab bij patiënten met eosinofiel astma geen belangrijke invloed heeft op de levenscyclus van neutrofielen en basofielen. Aan de andere kant hebben we bevestigd dat mepolizumab de hoeveelheid eosinofiele granulocyten in het sputum beperkt. Dit effect wordt veroorzaakt door: i minder aanmaak van eosinofiele voorlopers in het beenmerg, ii minder migratie van eosinofielen naar de weefsels en iii kortere levensduur van deze cellen in het sputum. Daarnaast, hebben we aangetoond dat ook bij de mens, IL-5 niet essentieel is voor de "normale" aanmaak van eosinofielen. Daarentegen is IL-5 wel essentieel in de verhoogde productie van eosinofielen, zoals het geval is bij eosinofiel astma.

Daarnaast hebben we een nieuwe en makkelijke methode bedacht om eosinofiele voorlopers in het beenmerg te identificeren. Met deze methode hebben we kunnen concluderen dat eosinopenie na acute inflammatie niet gepaard gaat met minder productie van eosinofielen in het beenmerg.

Ten slotte hebben we bevestigd dat de door dupilumab veroorzaakte eosinofilie, wordt veroorzaakt door verminderde migratie van deze cellen naar de huid in patiënten met constitutioneel eczeem. Dit effect is vermoedelijk niet anders voor de luchtwegen in het geval van astma. 


\section{Dankwoord}

De totstandkoming van dit proefschrift zou niet gelukt zijn zonder de hulp of steun van een heleboel mensen. Uiteraard, wil ik al deze mensen bedanken voor hun bijdrage.

Om te beginnen wil ik natuurlijk mijn promotor, professor Koenderman bedanken. Beste Leo (beter bekend als Lei-o, (L)EO of don Leo). Ik heb zelden een professor meegemaakt die zo gemakkelijk is in de omgang als jij. Daarnaast ben je een pro als het gaat om enthousiasme overbrengen. Wie had ooit gedacht dat ik zo geïnteresseerd zou zijn in de kinetiek van granulocyten. Dat heb ik toch echt wel aan jou te danken. Al snel, wist ik dat ik geen fout had gemaakt toen ik eind 2016 het definitieve besluit had genomen om mijn opleiding tot longarts te pauzeren en een nieuw avontuur aan te gaan als artsonderzoeker bij jou in de groep. Het waren absoluut drie topjaren, waarin ik veel heb geleerd over de wetenschap, maar ook enorm veel plezier heb gehad. Hopelijk kunnen we in de toekomst blijven samenwerken. Ik kijk er in ieder geval naar uit!

Nienke, hoewel jij dit niet officieel was, beschouwde ik jou een beetje als mijn copromotor. Vooral als het gaat om praktische zaken in het laboratorium kon ik vaak snel en gemakkelijk bij jou terecht. Bedankt hiervoor! Ik hoop ook met jou in de toekomst samen te werken.

Mijn dank gaat vanzelfsprekend ook uit naar alle patiënten van de Footstep studie, gezonde vrijwilligers van de minidonordienst en alle LPS studies en tot slot de patiënten met atopische dermatitis.

Ook de werknemers van GSK Nederland wil ik heel erg bedanken voor hun bijdrage aan mijn proefschrift, met name aan de Footstep studie. In het bijzonder wil ik Karin Velthove en Pauline Bloemen bedanken. Hopelijk hebben jullie net zo veel genoten van onze wetenschappelijke discussies als ik.

Verder wil ik ook mijn dank uiten aan de research team van de kinderlongziekten: Sabine, Hannah, Stephan, Margot, Julie en Myriam. Jullie hulp was essentieel in het succesvol voltooien van de Footstep studie.

Ook wil ik dank uiten aan de leden van de beoordelingscommissie van dit proefschrift: Prof. Taco Kuijpers, Prof. Paul Coffer, Prof. Kors van der Ent, Prof. Linde Meyaard en Aridaman Pandit.

Mijn paranimfen: Lucas en Abhinandan. Met jullie aan mijn zijde, moet die verdediging wel lukken, toch?

Lucas, hoewel wij vaak te lang bezig waren met koffiedrinken en het tegelijkertijd doornemen van alle Eredivisie wedstrijden, zou ik die tijd niet voor geen goud hebben willen missen. Helemaaal niet gek! Je bent behalve een collega, ook een goede vriend geworden. Toch vind ik het jammer dat jij je (nog) niet hebt ingeschreven (je weet wel wat ik bedoel). Ook bedankt dat je mijn wetenschappelijke resultaten altijd heb weten te downgraden: nee het is inderdaad geen Nature. 
Abhi: my brother from another mother. I think our friendship was inevitable, because we sat next to each other from the first moment I started at the LTI (now CTI), which for guys is often a recipe for friendship. No, we became friends, because we have the same (dark) humor and we share the love for philosophical discussions. I am going to miss the time at our desks. Just chill man and keep it real!

En dan uiteraard, wil ik ook alle mensen van het longenlab bedanken: om te beginnen wil ik een diepe buiging maken voor Corneli (beter bekend als Cornèli). Zonder jouw hulp was dit proefschrift minimaal 4 hoofdstukken dunner. Je hebt enorm veel voor mij gedaan: begeleiding in het lab, bloed opwerken, cellen sorteren en last but not least de meeste GC-MS samples opwerken. Hulde!

Erinke, ook jouw hulp was onmisbaar. Vooral in het lab heb ik veel van jou kunnen leren. Ook ik heb genoten van de vele discussies die we hebben gevoerd. Je had mij bijna overtuigd dat geneeskunde een veredelde hbo-opleiding is.

Selma, jouw hulp op het lab was ook zeker onmisbaar. Verder heb je me ook buiten het werk om, enorm geïnspireerd: ik zal helaas nooit binnen 20 minuten 5 kilometer kunnen rennen.

Pien, bedankt voor je hulp en droge humor. Hoofdstuk 3 van dit proefschrift is dankzij jouw inspanningen een stuk mooier geworden.

Deon, hoe belangrijk jouw bijdrage aan de Koenderman groep is, werd pas duidelijk toen je weg was. Het maken van Western Blots is een stuk lastiger als je er niet bent. Ik wil je uiteraard ook bedanken voor je heerlijke Saison bier en honing.

Tamar, mijn proefschrift had eigenlijk "Granulocyte kinetics in health and disease, part two" moeten heten. Daarmee, is het denk ik duidelijk hoe belangrijk jouw werk was voor mijn proefschrift. Hopelijk kunnen we in de toekomst nog vaak samenwerken.

De rest van de Koenderman groep wil ik uiteraard niet vergeten: Roy en Lilian (beter bekend als LilRoy), Karin, Eva, Danny, Na, Lucie, Tom Vos, Tom van der Most, Fabiënne, Giulio, Wiebe en Suus. Naast jullie hulp in het lab, hebben jullie allen ervoor gezorgd dat ik elke dag weer uitkeek naar de lunch- en koffiemomenten. Bedankt daarvoor.

De co-auteurs van de verschillende hoofdstukken die ik nog niet heb genoemd, verdienen ook mijn dank: Guus Leijte, Mathijs Kox, Niklas Bruse, Prof. Peter Pickkers, Falco Hietbrink, Daphne Bakker, Judith Thijs, Prof. Marjolein De Bruin-Weller, Edward Knol, Kiki Tesselaar, José Borghans, Julia Drylewicz en Saar van Nederveen-Bendien.

Ook de collega's van de longziekten wil ik graag bedanken voor alle hulp met de inclusie van de astmapatiënten. In het bijzonder: René Schweizer, Hans Kemming, Margriet Stellingwerf, Anne van Lindert, Bart Hilvering, Bart Luijk en Regina Hofland.

Dan de studenten die ik begeleid heb: Naomi en Daan, bedankt voor jullie werk aan hoofdstuk 5. Zonder jullie hulp had ik nog minder begrepen van de mysterieuze CXCR4 receptor. En Daan (parttime Daan), ik verwacht wel dat jij met alle antwoorden komt aan het einde van je stage. Grapje, ik ben een trotse begeleider. 
Mijn kamergenoten wil ik ook bedanken. Rianne Rijken, ik ga je gekke geluiden en imitaties van fietsopa nog missen. Nila, bedankt dat ik een aantal keer met jou naar de film mocht. Anders had ik die fantastische Star Wars film moeten missen. Tiago, thanks for being the only person to laugh at my bad jokes.

Dan de mensen met wie ik vaak genoeg op de vrijdagmiddag heb mogen borrelen: Marlot, Rianne Scholman, Lotte, Maarten, Sarah, Alsya, Mariona, Ellen, Janneke, Akashdip, Matevz, Mareille, Nanette en Renée. Bedankt voor de gezellige tijden en heerlijke gin en tonics.

Uiteraard wil ik de bijzondere borrelaars: Mo, Thijs, Lucas en Yesper (beter bekend als de borrelbro's) niet vergeten. Bedankt voor alle gezellige gesprekken over de wetenschap, sport en het leven. Parkie dan maar?

Verder wil ik de overige CTI werknemers bedanken, die ik niet eerder heb genoemd: Sigrid, Jorg, Bas, Kristin, Saskia, Yvonne, Gerrit, Jeroen en Pien.

Dan zijn er nog mensen buiten mijn werk, die ik ook graag wil noemen, omdat zij een belangrijke rol spelen in mijn leven.

Allereerst mijn twee broertjes: Salwan en Nur. Ik ben trots op jullie beiden. Vergeet niet dat onze koppigheid ons alle drie zo ver heeft gebracht.

Dan mijn ouders. Onderstaand bericht komt waarschijnlijk beter tot zijn recht in het Arabisch.

مثبحأ .ي نوروخف مكلعجا نأ لمآ .تققحام قيقحت ىلإ ةايحلا هذه يف مكتارايتخا تدأ ، يبأ و يمأ

Lieve, Carlijn, hoewel wij elkaar ontmoetten op een voor mij niet zo gunstig moment: laatste jaar van mijn PhD, kan ik wel zeggen dat je een heerlijke afleiding was en bent. Ik hou van je en ik kijk uit naar ons leven samen. 


\section{Curriculum vitae}

Marwan Hassani was born on the $3^{\text {rd }}$ of October 1988 in Baghdad, Iraq. In 1994 he moved together with his parents and brother to the Netherlands. He grew up in Bergen op Zoom, Noord-Brabant where he also finished his secondary education at R.S.G. 't Rijks.

In 2006, he started studying Medicine at the Utrecht University, where he obtained his bachelor's degree Cum Laude in 2009. In 2012 he obtained his Medical degree.

From 2013 to 2016 he worked as a resident in multiple hospitals in the Netherlands:

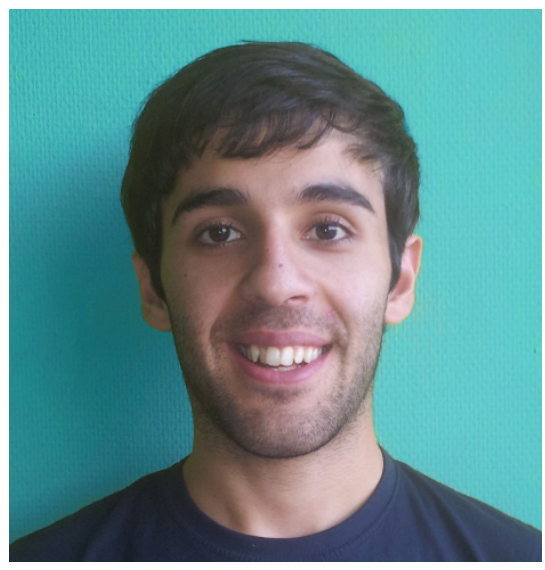
Maxima Medisch Centrium in Veldhoven, Diakonessenhuis in Utrecht, Spaarne Gasthuis in Hoofddorp and University Medical Center in Utrecht.

In 2016 he started his training in Respiratory Medicine at the University Medical Center in Utrecht.

In 2017, he was granted an opportunity to attain his PhD on the subject of human granulocyte kinetics. This project was under the supervision of Prof. Leo Koenderman. The results of this project are reported in this thesis.

In 2020, Marwan resumed his training in Respiratory Medicine. Currently he works at the Sint Antonius Hospital in Nieuwegein as a registrar in Internal Medicine. 

